\title{
Dose measurement in heterogeneous phantoms with an extrapolation chamber
}

François DeBlois

Physics Department

McGill University, Montreal

2001

A thesis submitted to the Faculty of Graduate Studies in Research in partial fulfillment of the requirements for the degree of Doctor of Philosophy 
National Library
of Canada

Acquisitions and Bibliographic Services

395 Wellington Street Ottawa ON K1A ON4 Canada
Bibliothèque nationale du Canada

Acquisitions et services bibliographiques

395. nue Wellington Ottawa ON K1A ON4 Canada
The author has granted a nonexclusive licence allowing the National Library of Canada to reproduce, loan, distribute or sell copies of this thesis in microform, paper or electronic formats.

The author retains ownership of the copyright in this thesis. Neither the thesis nor substantial extracts from it may be printed or otherwise reproduced without the author's permission.
L'auteur a accordé une licence non exclusive permettant à la Bibliothèque nationale du Canada de reproduire, prêter, distribuer ou vendre des copies de cette thèse sous la forme de microfiche/film, de reproduction sur papier ou sur format électronique.

L'auteur conserve la propriété du droit d'auteur qui protège cette thèse. $\mathrm{Ni}$ la thèse ni des extraits substantiels de celle-ci ne doivent être imprimés ou autrement reproduits sans son autorisation. 


\begin{abstract}
A hybrid phantom-embedded extrapolation chamber (PEEC) made of Solid Water ${ }^{\mathrm{TM}}$ and bone-equivalent material was used for determining absolute dose in a boneequivalent phantom irradiated with clinical radiation beams (cobalt-60 gamma rays; 6 and $18 \mathrm{MV}$ x-rays; and 9 and $15 \mathrm{MeV}$ electrons). The dose was determined with the SpencerAttix cavity theory, using ionization gradient measurements and an indirect determination of the chamber air-mass through measurements of chamber capacitance. The air gaps used were between 2 and $3 \mathrm{~mm}$ and the sensitive air volume of the extrapolation chamber was remotely controlled through the motion of the motorized piston with a precision of \pm 0.0025 $\mathrm{mm}$. The collected charge was corrected for ionic recombination and diffusion in the chamber air volume following the standard two-voltage technique. Due to the hybrid chamber design, correction factors accounting for scatter deficit and electrode composition were determined and applied in the dose equation to obtain dose data for the equivalent homogeneous bone phantom. Correction factors for graphite electrodes were calculated with Monte Carlo techniques and the calculated results were verified through relative air cavity dose measurements for three different polarizing electrode materials: graphite, steel, and brass in conjunction with a graphite collecting electrode. Scatter deficit, due mainly to loss of lateral scatter in the hybrid chamber, reduces the dose to the air cavity in the hybrid PEEC in comparison with full bone PEEC from 0.7 to $~ 2 \%$ depending on beam quality and energy. In megavoltage photon and electron beams, graphite electrodes do not affect the dose measurement in the Solid Water ${ }^{\mathrm{TM}}$ PEEC but decrease the cavity dose by up to $5 \%$ in the bone-equivalent PEEC even for very thin graphite electrodes $(<0.0025 \mathrm{~cm})$. The collecting electrode material in comparison with the polarizing electrode material has a larger effect on the electrode correction factor; the thickness of thin electrodes, on the other hand, has a negligible effect on dose determination. The uncalibrated hybrid PEEC is an accurate and absolute device for measuring the dose directly in bone material in conjunction with appropriate correction factors determined with Monte Carlo techniques.
\end{abstract}




\section{Résumé}

Une chambre à extrapolation (PEEC), incrustée dans le matériel de mesure de composition hybride, "Solid Water TM" et "os-équivalent", a été utilisée pour déterminer la dose de façon absolue dans un fantôme de composition équivalente à l'os irradié avec des faisceaux de rayonnements cliniques (rayons gamma de cobalt-60; rayons $x$ de 6 et $18 \mathrm{MV}$ et rayons bêta de 9 et $15 \mathrm{MeV}$ ). La dose absorbée a été déterminée à l'aide de la théorie de Spencer-Attix en utilisant les conditions de cavité de Bragg-Gray ainsi qu'une détermination indirecte de la masse d'air de la cavité par une mesure de capacitance et du gradient d'ionisation. Le volume d'air pratique de la chambre, variant de 2.0 à $3 \mathrm{~mm}$, était contrôlé à distance par le mouvement d'un piston motorisé à une précision de mouvement linéaire de $\pm 0.0025 \mathrm{~mm}$. La charge mesurée a été corrigée pour la perte due à la recombinaison et à la diffusion ionique suivant la technique des deux-tension. En raison de la conception de la chambre, des facteurs de correction pour l'effet du déficit en rayonnement diffus et l'effet causé par la présence des électrodes sur la dose ont été déterminés et appliqués à l'équation de dose afin d'obtenir une mesure de dose dans un fantôme homogène équivalent à l'os. Les facteurs de correction pour des électrodes de graphite ont été calculés avec des techniques de Monte Carlo et vérifiés par des mesures relatives de la dose absorbée par la cavité d'air pour trois différents matériaux d'électrode de polarisation: le graphite, l'acier et le laiton, tout en utilisant une électrode de graphite pour la collection des ions. Le déficit en rayonnement diffus est principalement causé par la différence de rayonnement diffus transversal dans la chambre hybride et ce déficit entraîne une différence de dose mesurée entre les deux géométries de l'ordre de 0.7 à $~ 2 \%$ selon la qualité et l'énergie des faisceaux. Pour les faisceaux de photons et d'électrons à hautes énergies, les électrodes de graphite n'affectent pas la mesure de dose dans le matériel Solid Water ${ }^{\mathrm{TM}}$ mais diminuent la dose à la cavité d'air jusqu' à $5 \%$ dans la PEEC hybride et ce, même pour les électrodes très minces de graphite $(<0,0025 \mathrm{~cm})$. La composition de l'électrode de collection a un plus grand effet que le matériel de l'électrode de polarisation sur le facteur de correction d'électrode; l'épaisseur des électrodes minces, $d$ autre part, a un effet négligeable sur la détermination de la dose. La PEEC hybride non-calibrée est un dispositif précis et absolu pour mesurer la dose directement dans l'os à la condition d'utiliser des facteurs de correction correctement calculés avec les techniques Monte Carlo. 


\section{Original Contribution}

The thesis deals with absolute dose measurement in a bone-equivalent phantom and contains several experimental and theoretical approaches that represent an original contribution to current knowledge in clinical radiation dosimetry.

We are the first group to report absolute dose measurements directly in boneequivalent material, a biological material other than tissue, with the use of a phantomembedded extrapolation chamber (PEEC). National and international dosimetry protocols are not suitable for dose determination in non-tissue-like biological materials, since they require the use of many correction factors that are only available for water-like phantoms used in conjunction with ionization chambers calibrated in standards laboratories. Our dose determination approach in bone, based on the Spencer-Attix cavity theory, is similar to the approach followed previously for dose measurements with PEEC in Solid Water ${ }^{\mathrm{TM}}$ by Zankowski. We extended the design of the Solid Water ${ }^{\mathrm{TM}}$ chamber to obtain a hybrid boneequivalent PEEC consisting of the original PEEC with the following modifications: (1) a bone entrance window, supporting the polarizing electrode, and (2) a disc of bone material below the collecting electrode of sufficient thickness to produce backscatter radiation equivalent to that obtained by a chamber fully embedded in bone-equivalent material.

We carried out an original experimental and theoretical study of various possible effects which could adversely affect dose-to-bone measurements. With an incorporation of scatter and electrode correction factors to dose-to-bone measured data we achieved a perfect agreement between experimental dose-to-bone data and data calculated with Monte Carlo techniques. Our hybrid PEEC is thus capable of yielding accurate absolute dose to bone-equivalent material without requiring a standards laboratory calibration factor. The dose determination procedure is based on the chamber signal after incorporating a correction factor determined through Monte Carlo techniques. The correction factor accounts for scatter perturbation in the hybrid PEEC, as well as for effects of graphite electrodes affecting the dosimetric signal measured in the chamber air cavity.

The original Monte Carlo model of the hybrid PEEC with various cavity configurations enabled us to evaluate several dose correction factors. Five clinical megavoltage beams were modeled with Monte Carlo simulations to provide radiation beams for the 
actual chamber simulations. This enabled us to calculate the realistic mean mass restricted stopping power ratios, bone to air, for the respective beams, required by the dose equation.

Through Monte Carlo calculations, we verified Zankowski's assumption that graphite electrodes for Solid Water ${ }^{\mathrm{TM}}$ PEEC have no effect on the measured dose-to-air cavity obviating the use of electrode correction factors. For the hybrid bone PEEC we demonstrated that the graphite electrodes produce a non-negligible effect and corrections have to be applied to account for their presence. We also showed that a change of electrode thickness produces a negligible effect on the air cavity dose for thin electrodes and that the polarizing electrode in comparison with the collecting electrode produces a greater effect on the measured air cavity dose. Monte Carlo techniques also demonstrated that lateral scatter is different in the hybrid bone PEEC than in the PEEC entirely made of bone material.

We also automated the extrapolation chamber by remotely controlling the displacement of the electrodes with a computer program. A precise stepping motor attached to a micrometer moves the piston position which changes the sensitive air cavity volume of the PEEC chamber. This allowed us to simplify the data acquisition process. Acquisition programs using the IEEE-488 interface were also developed to control the electrometer and power supply that are used for PEEC measurements.

We investigated the validity of the saturation curve model developed by Zankowski for pulsed beams with several ionization chambers (NE2517, Capintec PR-06, Holt, and PEEC). For ionization chambers exposed to pulsed megavoltage photon and electron beams, the linearity of the $1 / I$ and $1 / V$ plot breaks down in the polarizing voltage range where chambers are normally operated. The breakdown is caused by charge multiplication in the chamber sensitive volume and by leakage currents produced by radiation in the chamber stem, connector, and cable. The magnitude of this effect varies from one chamber to another and is also strongly field size dependent.

Depending on the particular ionization chamber model, the breakdown in the linearity between $1 / I$ and $1 / V$ may cause an overestimate of the radiation beam output by $\sim 0.7 \%$ for a $10 \times 10 \mathrm{~cm}^{2}$ field and by a few percent for large radiation fields when the standard "two-voltage" technique is used for determination of the saturation current. Therefore the "two-voltage" technique should be used with caution. 


\section{Acknowledgments}

I would like to express my endless gratitude to my supervisor, Dr. Ervin B. Podgorsak for his guidance, support and understanding throughout the years. Without the excellent working environment he has created the completion of this project would not have been possible.

The various components of the apparatus employed in this project were created and maintained by many people. I would like to thank Shoukry Aboulehaf for machining the various parts which were added to the phantom-embedded extrapolation chamber. I would like to acknowledge Joe Larkin and Pierre Léger who helped with the electronic aspects of the project, and especially Gilles Parent who designed the stepping motor control card.

I express endless thanks to my fellow students and friends Dr. Corey Zankowski, Dr. Arthur Curtin-Savard, Dr. Tony Falco, and Wamied Abdel-Rahman. All of them not only helped me out in various aspects of the project but also cheered me up and understood the various moods that one goes through in completing a $\mathrm{Ph}$. D. project.

I would like to thank my parents Carmen and Pierre who always encouraged studying and pursuing one's dreams. I would like to acknowledge my sisters Geneviève and Anne-Marie and my in-law parents Denise and Jean who cheered me up and always believed in me.

Last but not least, I want to thank my beloved wife Danielle for all her love, support, understanding, and patience during the past years, while I was away at night, studying, taking measurements, fixing up computers or writing the thesis. A special thanks to my son Julien for giving me so much joy and energy which helped me complete this project. 


\section{Table of Contents}

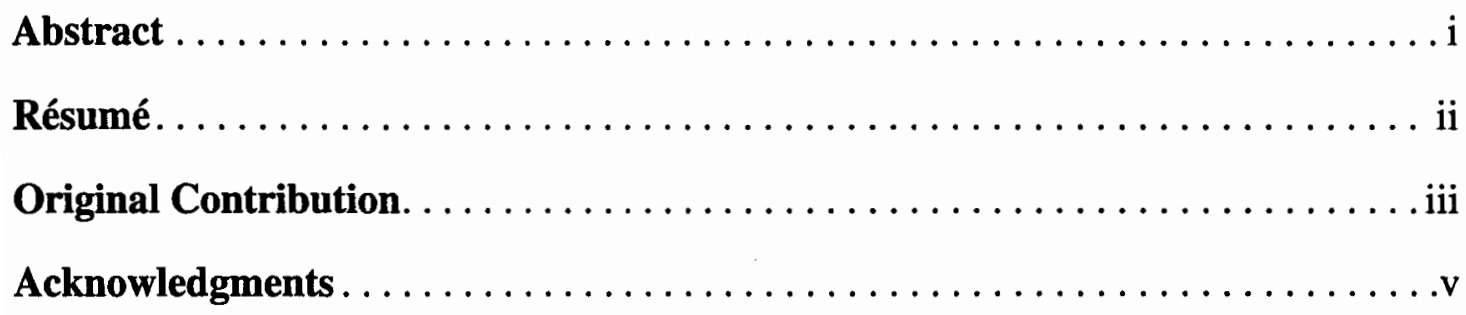

List of abbreviations and symbols $\ldots \ldots \ldots \ldots \ldots \ldots \ldots \ldots \ldots \ldots \ldots \ldots \ldots$

\section{Chapter 1}

Introduction $\ldots \ldots \ldots \ldots \ldots \ldots \ldots \ldots \ldots \ldots \ldots \ldots$

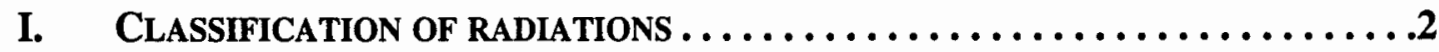

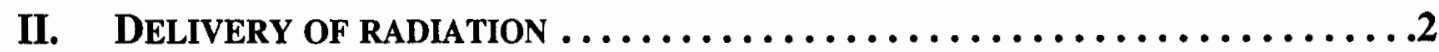

III. TREATMENT PLANNING AND VERIFICATION $\ldots \ldots \ldots \ldots \ldots \ldots \ldots \ldots, \ldots$

IV. RADIATION DOSIMETRY TECHNIQUES $\ldots \ldots \ldots \ldots \ldots \ldots \ldots \ldots \ldots \ldots \ldots$

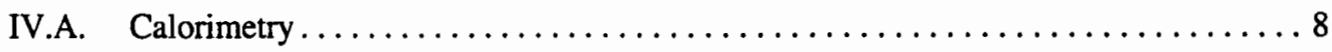

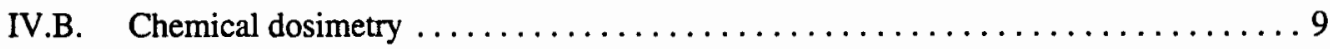

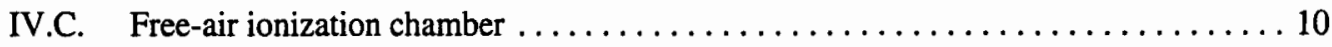

IV.D. Phantom-embedded extrapolation chamber (PEEC) $\ldots \ldots \ldots \ldots \ldots \ldots \ldots \ldots \ldots \ldots \ldots$

V. MOTIVATION FOR THIS WORK $\ldots \ldots \ldots \ldots \ldots \ldots \ldots \ldots \ldots \ldots \ldots \ldots \ldots \ldots \ldots$

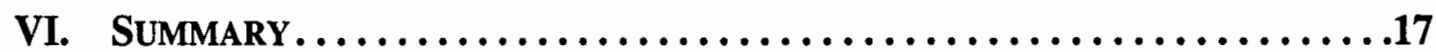

\section{Chapter 2}

Interactions of photons with matter $\ldots \ldots \ldots \ldots \ldots \ldots \ldots \ldots 22$

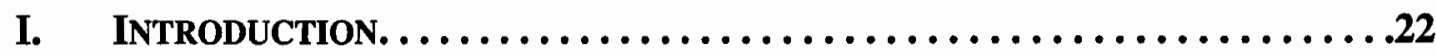

I.A. Types of indirectly ionizing photon radiations . . . . . . . . . . . . . 22

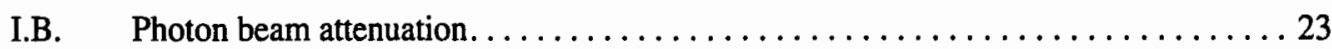

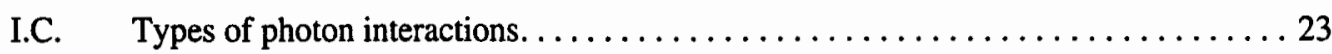

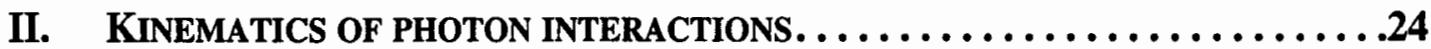

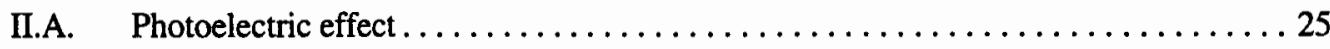

II.B. Compton effect (incoherent scattering) $\ldots \ldots \ldots \ldots \ldots \ldots \ldots \ldots \ldots \ldots \ldots \ldots$

II.C. Pair and Triplet production $\ldots \ldots \ldots \ldots \ldots \ldots \ldots \ldots \ldots \ldots \ldots \ldots \ldots \ldots \ldots \ldots$

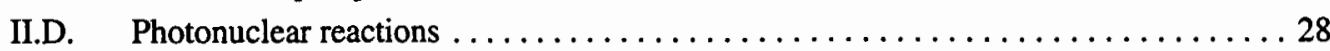




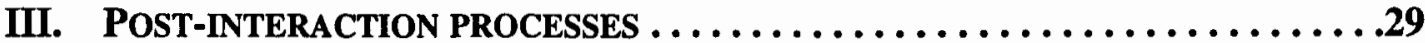

III.A. Characteristic radiation. . . . . . . . . . . . . . . . . . . . . . . . . . 29

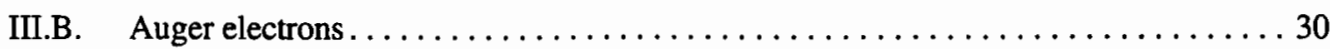

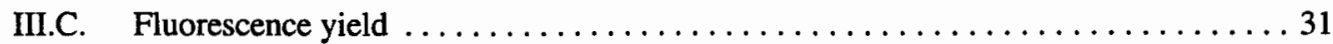

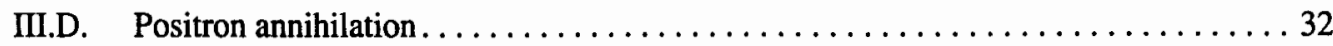

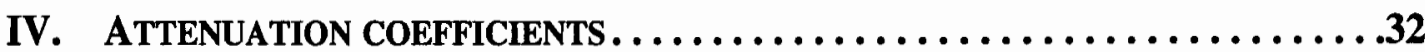

IV.A. Cross sections and attenuation coefficients $\ldots \ldots \ldots \ldots \ldots \ldots \ldots \ldots \ldots \ldots \ldots \ldots$

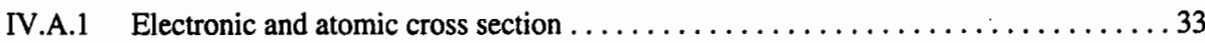

IV.A.2 Linear attenuation coefficient. . . . . . . . . . . . . . . . . . . . . . . . 34

IV.A.3 Mass attenuation coefficient. . . . . . . . . . . . . . . . . . . . . . . . 35

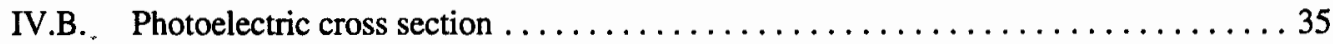

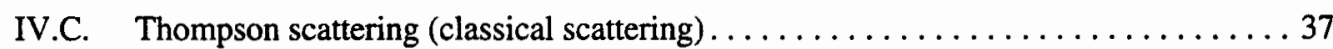

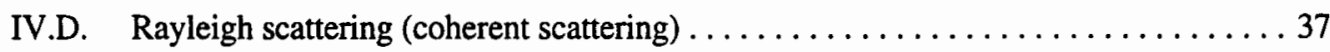

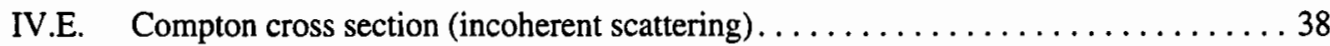

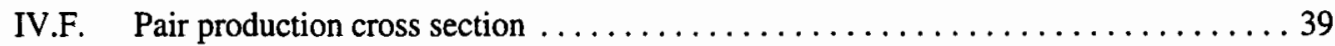

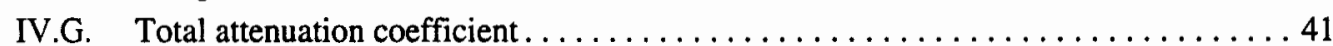

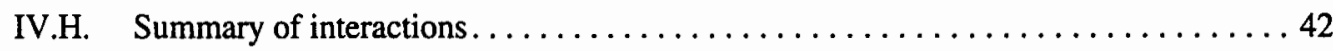

IV.I. Mass energy-transfer coefficient. ........................ 44

IV.I.1 Energy transfer in photoelectric effect .......................... 45

IV.I.2 Energy transfer in Compton effect ............................ 45

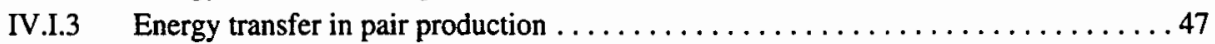

IV.I.4 Total mass energy transfer coefficient $\ldots \ldots \ldots \ldots \ldots \ldots \ldots \ldots \ldots \ldots \ldots 47$

IV.J. Total mass energy absorption coefficient $\ldots \ldots \ldots \ldots \ldots \ldots \ldots \ldots \ldots . \ldots 48$

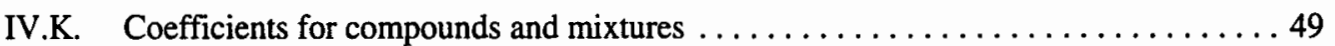

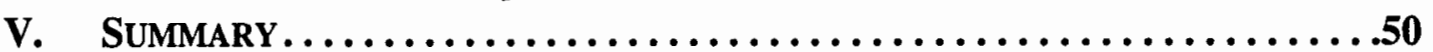

\section{Chapter 3}

Interactions of electrons with matter . . . . . . . . . . . . 54

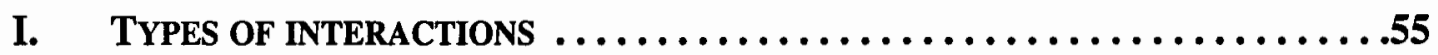

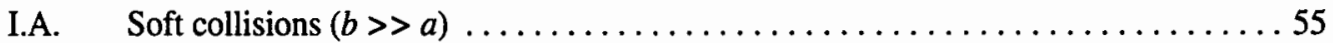

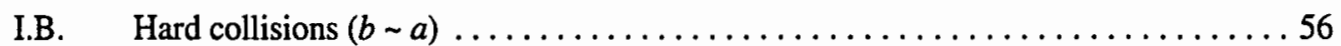

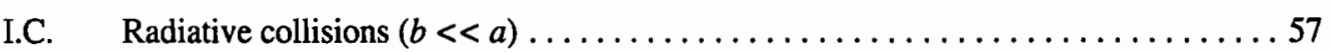

II. ENERGY TRANSFER TO THE MEDIUM $\ldots \ldots \ldots \ldots \ldots \ldots \ldots \ldots \ldots \ldots \ldots \ldots$

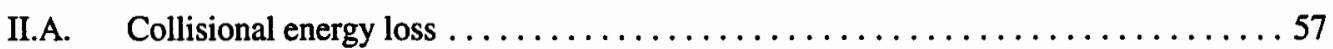

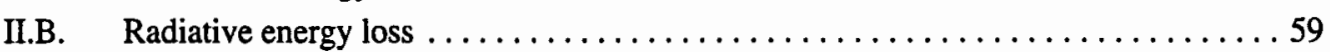

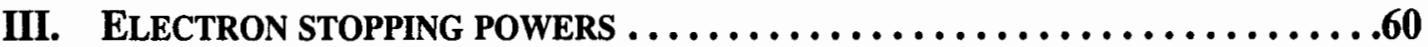

III.A. Mass collisional stopping power. ......................... 61

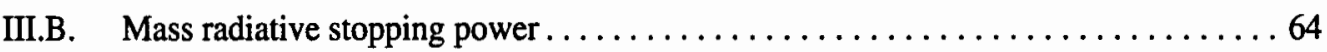

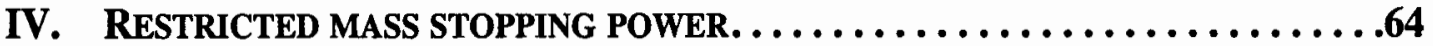

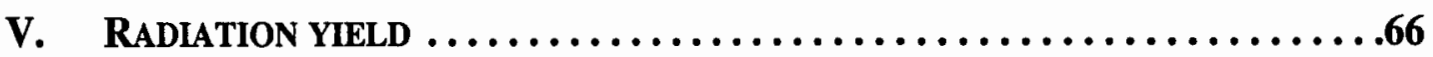

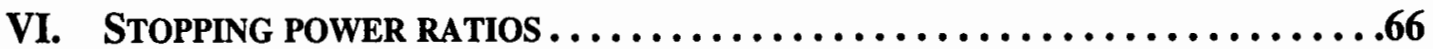

VII. STOPPING POWER OF COMPOUNDS $\ldots \ldots \ldots \ldots \ldots \ldots \ldots \ldots \ldots \ldots \ldots \ldots \ldots$ 
VIII. ENERGY ABSORBED BY THE MEDIUM $\ldots \ldots \ldots \ldots \ldots \ldots \ldots \ldots \ldots \ldots \ldots$

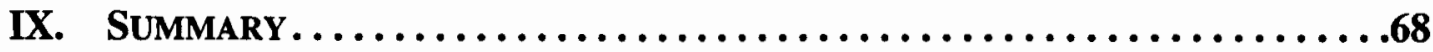

\section{Chapter 4}

Measurement of absorbed dose $\ldots \ldots \ldots \ldots \ldots \ldots \ldots \ldots \ldots$

I. BASIC QUANTITIES USED IN RADIATION DOSIMETRY. . . . . . . . . 72

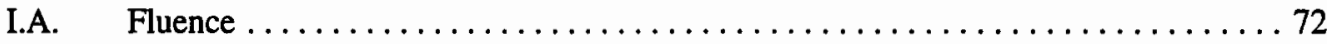

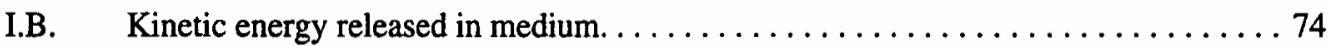

I.C. Absorbed dose. . . . . . . . . . . . . . . . . . . . . . . . . . . . 75

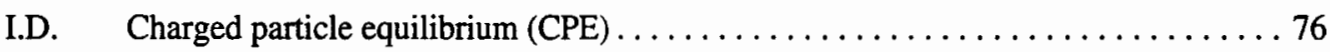

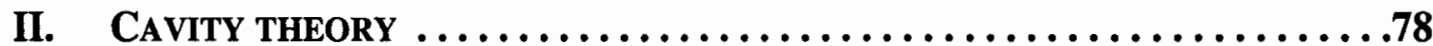

II.A. Bragg-Gray cavity theory $\ldots \ldots \ldots \ldots \ldots \ldots \ldots \ldots \ldots \ldots \ldots \ldots \ldots \ldots \ldots \ldots \ldots$

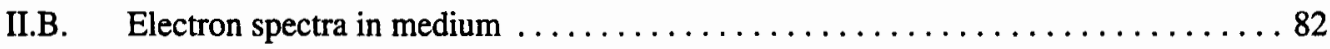

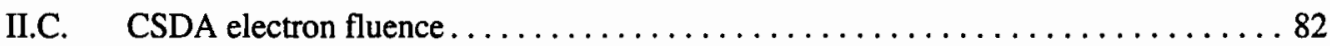

II.D. Electron spectra including secondary electrons $\ldots \ldots \ldots \ldots \ldots \ldots \ldots \ldots . \ldots . \ldots . \ldots . \ldots$

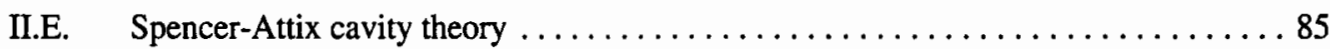

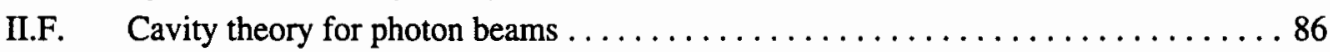

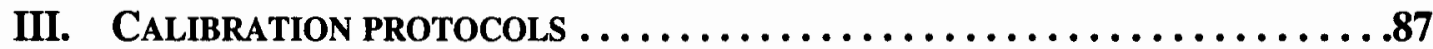

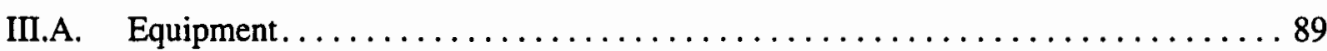

III.B. Beam quality specification $\ldots \ldots \ldots \ldots \ldots \ldots \ldots \ldots \ldots \ldots \ldots \ldots$

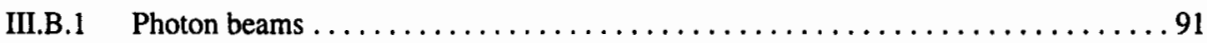

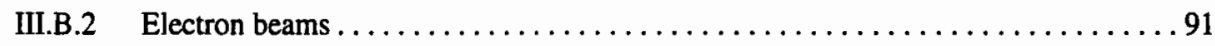

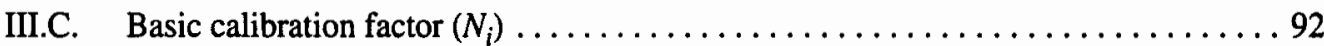

III.D. Chamber reading correction factors ........................... 94

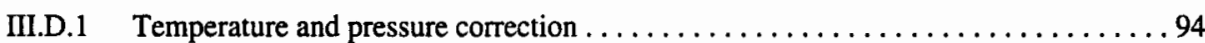

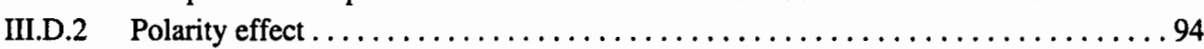

III.D.3 Ionic recombination correction factor $P_{\text {ion }} \ldots \ldots \ldots \ldots \ldots \ldots \ldots \ldots \ldots \ldots \ldots$

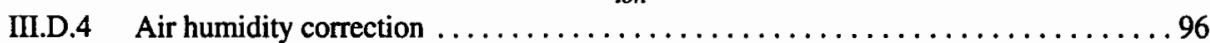

III.D.5 Leakage. . . . . . . . . . . . . . . . . . . . . . . . . . . . 96

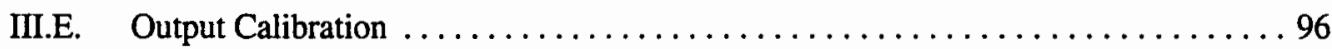

III.E.1 Output calibration of low energy (superficial) $\mathrm{x}$-ray beams

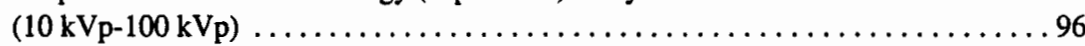

III.E.2 Output calibration of medium energy (orthovoltage) $x$-ray

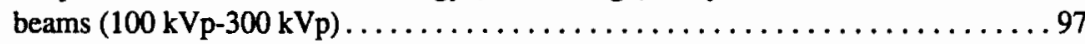

III.E.3 Output calibration of megavoltage photon beams based upon air-kerma

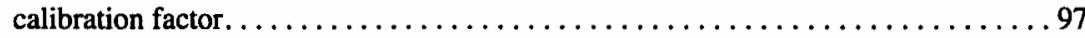

III.E.4 Output calibration of megavoltage electron beams based upon air-kerma calibration factor. . . . . . . . . . . . . . . . . . . . . . . . . . . 98

III.E.5 Output calibration of megavoltage photon beams based upon dose to water calibration factor (AAPM TG-51 protocol) . . . . . . . . . . . . 99

III.E.6 Output calibration of megavoltage electron beams based upon dose to water calibration factor (AAPM TG-51 protocol) $\ldots \ldots \ldots \ldots \ldots \ldots \ldots \ldots \ldots \ldots$

IV. SUMMARY 


\section{Chapter 5}

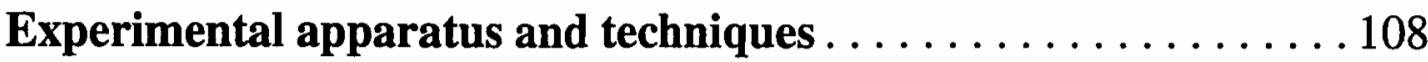

I. Phantom-embedded extrapolation Chamber $\ldots \ldots \ldots \ldots \ldots \ldots \ldots$

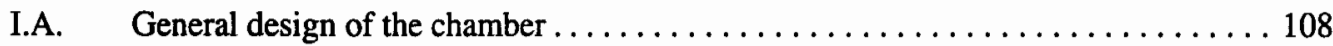

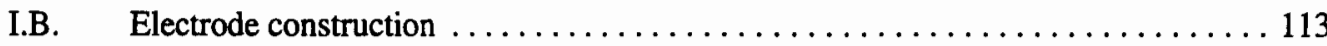

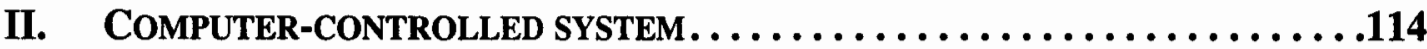

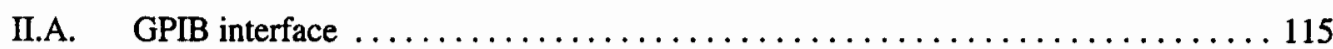

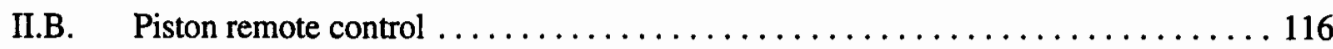

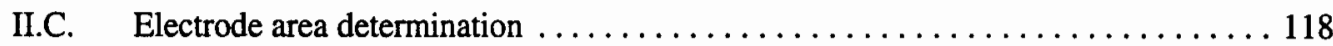

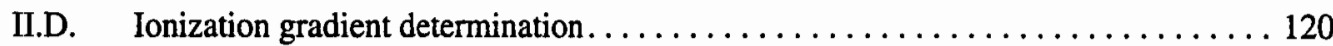

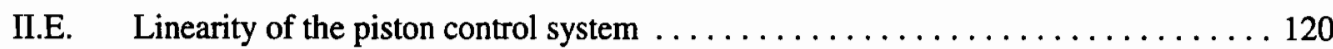

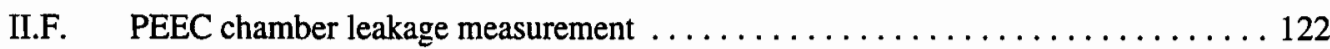

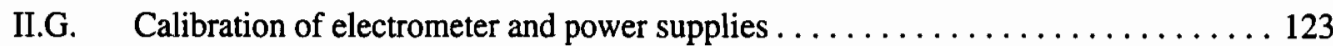

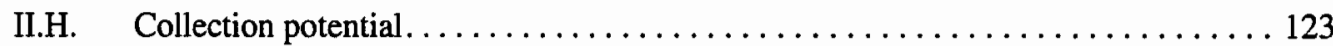

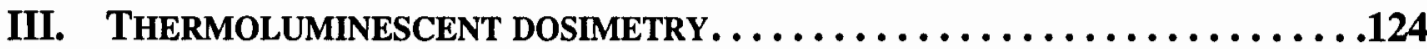

IV. MEASUREMENT OF DOSE TO BONE WITH THE PEEC $\ldots \ldots \ldots \ldots \ldots \ldots .124$

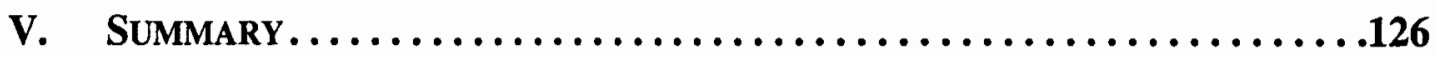

\section{Chapter 6}

Monte Carlo calculations . . . . . . . . . . . . . . . . . . 130

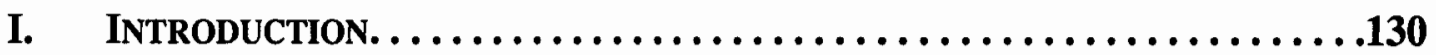

II. EGS MONTE CaRLo CODE SySTEM $\ldots \ldots \ldots \ldots \ldots \ldots \ldots \ldots \ldots \ldots . . .132$

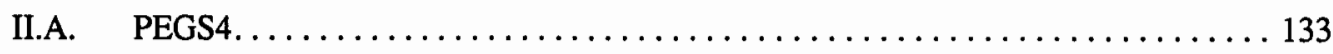

II.B. $\quad$ EGS4/EGSnrc . . . . . . . . . . . . . . . . . . . . . . . . . . . . 133

II.B.1 EGS4/EGSnrc User Codes ................................... 137

II.B.2 Variance reduction techniques ............................ 137

III. BEAM MONTE CARLo CODE SYSTEM ....................139

IV. Monte Carlo: Montreal GeNERal Hospital

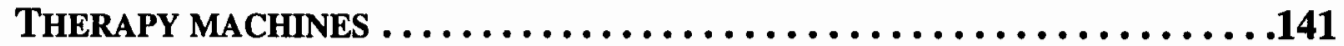

IV.A. Varian Clinac-18........................................ 142

IV.B. Clinac-2300 C/D ........................................... 146

IV.C. Theratron T-780 (Cobalt-60 machine) ........................ 149

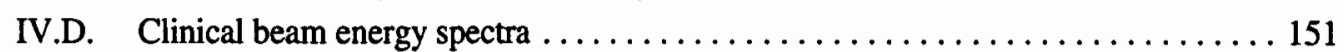

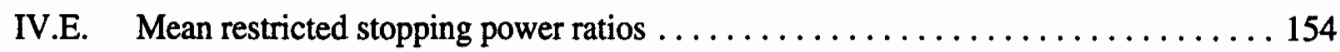

V. PEEC Monte CaRLo MOdEL $\ldots \ldots \ldots \ldots \ldots \ldots \ldots \ldots \ldots \ldots \ldots . \ldots \ldots$

VI. Medical Physics Unit Monte Carlo computer

CLUSTER.........................................155 


\section{Chapter 7}

Collection efficiency for ionization chambers in pulsed beams . . . . 162

I. INTRODUCTION.......................................

II. MATERIAL AND METHODS..............................164

II.A. Ion collection efficiency for pulsed radiation beams $\ldots \ldots \ldots \ldots \ldots \ldots \ldots$

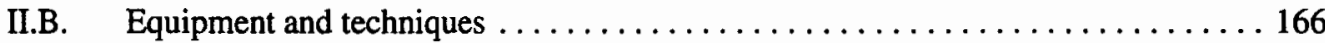

III. RESULTS AND DISCUSSION. ............................169

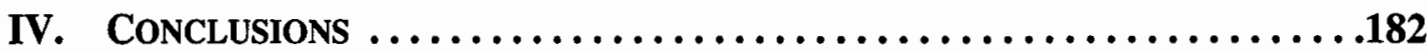

\section{Chapter 8}

Measurement of absorbed dose in bone-equivalent material $\ldots \ldots 186$

I. INTRODUCTION. ....................................186

II. ABSORBED DOSE DETERMINATION WITH THE PEEC $\ldots \ldots \ldots \ldots \ldots \ldots . .187$

III. Precision of dose determination with the PEEC $\ldots \ldots \ldots \ldots \ldots . .188$

III.A. Ionization gradient $d Q / d z \ldots \ldots \ldots \ldots \ldots \ldots \ldots \ldots \ldots \ldots \ldots \ldots \ldots \ldots, \ldots \ldots \ldots$

III.B. Air density $\rho \ldots \ldots \ldots \ldots \ldots \ldots \ldots \ldots \ldots \ldots \ldots \ldots \ldots \ldots \ldots \ldots \ldots$

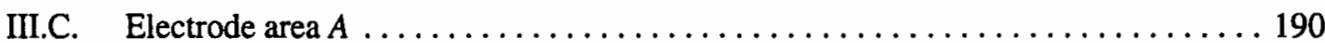

III.D. Mean energy required to produce an ion pair in air . . . . . . . . . . . . . . 190

III.E. Mean restricted mass stopping power ratios $\ldots \ldots \ldots \ldots \ldots \ldots \ldots \ldots \ldots \ldots$

III.F. Overall precision of the PEEC . . . . . . . . . . . . . . . . . . . 191

IV. ABSORBED DOSE DETERMINATION WITH

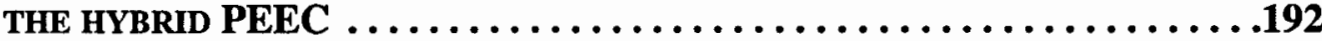

IV.A. Scatter correction $\ldots \ldots \ldots \ldots \ldots \ldots \ldots \ldots \ldots \ldots \ldots \ldots \ldots \ldots \ldots$

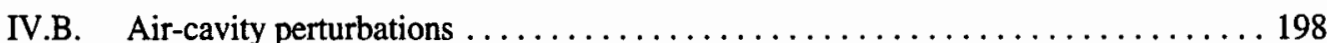

IV.C. Correction for chamber wall . . . . . . . . . . . . . . . . . . . . . . . . . . . 199

IV.D. Correction for electrode perturbation $\ldots \ldots \ldots \ldots \ldots \ldots \ldots \ldots \ldots \ldots \ldots \ldots \ldots$

IV.D.1 Evaluation of graphite electrodes for the Solid Water ${ }^{\mathrm{TM}}$ PEEC. . . . . . . . 200

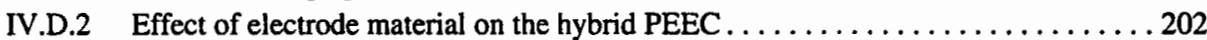

IV.D.3 Experimental verification of electrode material effect............... 204

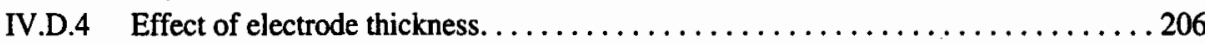

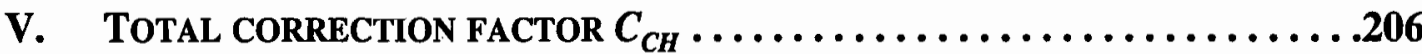

VI. DOSE MEASUREMENT IN BONE WITH THE HYBRID PEEC. . . . . . ....207

VII. Conclusions....................................209 


\section{Chapter 9}

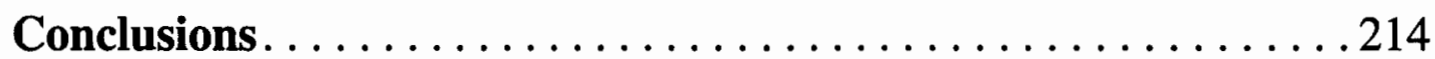

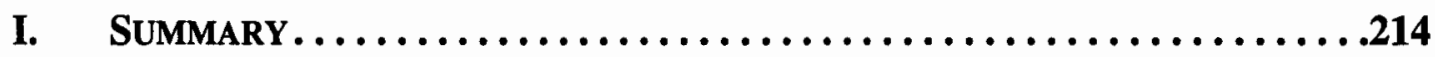

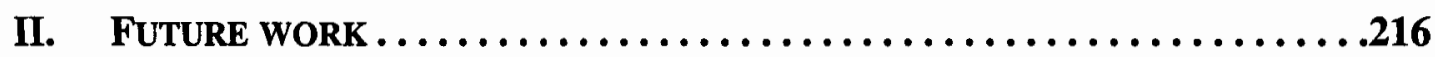

Appendix 1

IEEE-488 instrument control. . . . . . . . . . . . . . . . . . . . 218

Appendix 2

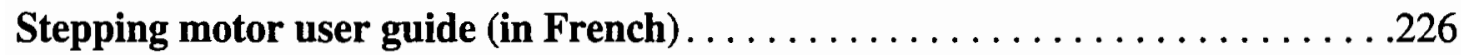

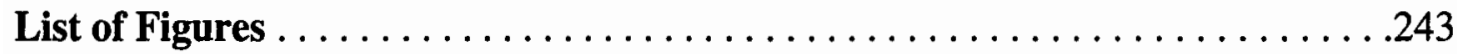

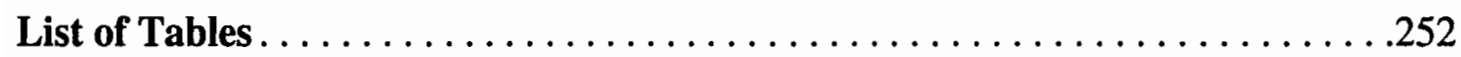

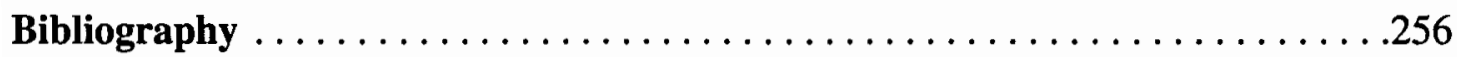




\section{List of abbreviations and symbols}

$\begin{array}{ll}\text { 3-D } & \text { three dimensional } \\ \beta^{-} & \text {beta radiation (e-) } \\ \gamma & \text { gamma radiation } \\ \text { AAPM } & \text { American Association of Physicists in Medicine } \\ \text { BEAM } & \text { EGS-based user code to model medical linacs } \\ \text { beamdp } & \text { BEAM distribution program to analyze the content of a phase-space } \\ \text { BG } & \text { file } \\ \text { Bremsstrahlung } & \text { Bragg-Gray } \\ C_{c h} & \text { emission of photons by charged particles } \\ \text { CH } & \text { total dose correction factor for hybrid PEEC } \\ \text { Co-60 } & \text { Condensed History } \\ \text { CM } & \text { Cobalt-60 isotope } \\ \text { CPE } & \text { Component Module (for BEAM code) } \\ \text { CPU } & \text { Charged Particle Equilibrium } \\ \text { CSDA } & \text { Central Processing Unit } \\ \text { CT } & \text { Continuous Slowing Down Approximation } \\ C_{T, P} & \text { Computerized Tomography } \\ \text { DOSXYZ } & \text { correction factor for temperature and pressure } \\ \text { DOSRZnrc } & \text { cartesian-based ( } x \text {,y,z) EGS user code to calculate dose } \\ d Q / d z & \text { cylindrical-based ( } r, z) \text { EGS user code to calculate dose } \\ d_{m a x} & \text { ionization gradient } \\ \bar{E}_{a b} & \text { average energy absorbed } \\ & \end{array}$




\begin{tabular}{|c|c|}
\hline $\bar{E}_{t r}$ & average energy transferred \\
\hline e+ & positron \\
\hline e- & electron \\
\hline ECUT & electron cut-off energy \\
\hline EGS & Electron Gamma Shower \\
\hline EGS4 & EGS version 4.0 \\
\hline EGSnrc & EGS NRC version \\
\hline$h v$ & photon energy \\
\hline HVL & Half Value Layer \\
\hline IAEA & International Atomic Energy Agency (Vienna, Austria) \\
\hline ICRU & $\begin{array}{l}\text { International Commission on Radiation Units and Measurements } \\
\text { (Bethesda, MD) }\end{array}$ \\
\hline IEEE & Institute of Electrical and Electronics Engineers \\
\hline KERMA & Kinetic Energy Released in Medium $(K)$ \\
\hline$\kappa / \rho$ & pair production mass attenuation coefficient \\
\hline$k_{h}$ & humidity correction factor \\
\hline $\mathrm{K}-\mathrm{N}$ & Klein-Nishina \\
\hline$\lambda_{p}$ & parameter for ionic recombination \\
\hline$(\bar{L} / \rho)_{\text {med }_{2}}^{\text {med }_{2}}$ & mean restricted mass stopping power ratio medium 1 to medium 2 \\
\hline linac & linear accelerator \\
\hline$\mu / \rho$ & total mass attenuation coefficient \\
\hline$\mu_{t r} / \rho$ & total mass energy-transfer attenuation coefficient \\
\hline$\mu_{a b} / \rho$ & total mass energy-absorption coefficient \\
\hline $\mathrm{MC}$ & Monte Carlo \\
\hline MU & Monitor Unit \\
\hline $\mathrm{n}$ & neutron \\
\hline$N_{D}$ & dose calibration factor in water \\
\hline
\end{tabular}




\begin{tabular}{|c|c|}
\hline$N_{x}$ & exposure calibration factor \\
\hline$N_{k}$ & air-kerma in air calibration factor \\
\hline NIST & National Institute of Standards and Technology \\
\hline NQS & Network Queueing System \\
\hline NRC & National Research Council (Ottawa, Canada) \\
\hline$O A R$ & Off-Axis Ratio \\
\hline$\phi$ & photon fluence \\
\hline$\psi$ & photon energy fluence \\
\hline$\Phi$ & total electron fluence spectrum \\
\hline$\Phi(T)$ & differential electron fluence spectrum \\
\hline PCUT & Photon cut-off energy \\
\hline$P D D$ & Percent Depth Dose \\
\hline PEEC & Phantom-Embedded Extrapolation Chamber \\
\hline PEGS & Preprocessor for EGS \\
\hline phase-space file & $\begin{array}{l}\text { BEAM generated computer file containing list of particles with } \\
\text { their important physical characteristics }\end{array}$ \\
\hline$P_{i o n}$ & ionic recombination correction factor \\
\hline PRESTA & Parameter Reduced Electron Step Algorithm \\
\hline$r_{o}$ & classical electron radius $\left(2.818 \times 10^{-13} \mathrm{~cm}\right)$ \\
\hline$R_{50}$ & depth where dose is $50 \%$ of the maximum dose in electron beams \\
\hline$R_{100}$ & depth of maximum dose for electron beams \\
\hline$R p$ & practical electron range \\
\hline RFA & Radiation Field Analyzer \\
\hline RNG & Random Number Generator \\
\hline RTOG & Radiation Therapy Oncology Group (Philadelphia, PA) \\
\hline$e^{\sigma_{o}}$ & total Thompson cross section per electron $\left(6.65 \times 10^{-25} \mathrm{~cm}^{2} /\right.$ electron $)$ \\
\hline$\sigma / \rho$ & Compton mass attenuation coefficient \\
\hline
\end{tabular}




$\begin{array}{ll}(\bar{S} / \rho)_{m_{2} d_{2}}^{\text {med }_{2}} & \text { mean unrestricted mass stopping power ratio medium } 1 \text { to medium } 2 \\ \text { SI } & \text { International System of units } \\ \text { SLAC } & \text { Stanford Linear Accelerator Center } \\ S P R & \text { Stopping Power Ratio } \\ \text { MRSPR } & \text { Mean Mass Restricted Stopping Power Ratio } \\ \text { SPRRZnrc } & \text { cylindrical-based (r,z) EGS user code to calculate SPRR } \\ S S D & \text { Source-Surface Distance } \\ \text { SW } & \text { Solid Water }{ }^{\mathrm{TM}}, \text { water equivalent plastic material } \\ \tau / \rho & \text { photoelectric mass attenuation coefficient } \\ \text { TE } & \text { Track-End term for Spencer-Attix cavity theory } \\ \text { TG } & \text { AAPM Task Group reports } \\ \text { TLD } & \text { Thermoluminescence Dosimetry } \\ T P R & \text { Tissue-Phantom Ratio } \\ \text { TRS } & \text { IAEA Technical Report Series } \\ T V L & \text { Tenth Value Layer } \\ u & \text { parameter in Boag's equation for collection efficiency } \\ \bar{W} & \text { mean energy required to create an ion pair in air (33.97 J/C) } \\ X & \text { exposure (C/kg) } \\ Z & \text { atomic number }\end{array}$




\section{Chapter 1}

\section{Introduction}

The discoveries of $x$ rays and radioactivity by Röntgen in $1895^{1-4}$ and Becquerel in $1896^{5-7}$, respectively, marked the beginning of medical radiation physics. Medical physics is a branch of physics concerned with the application of physics to medicine, particularly in the diagnosis and treatment of human disease. The main areas of interest at present are in the treatment of cancer by ionizing radiation (radiation-oncology); in diagnostic imaging with $\mathrm{x}$ rays, ultrasound and nuclear magnetic resonance (diagnostic radiology); in diagnostic imaging with radioisotopes (nuclear medicine); and in the study of radiation hazards and radiation protection (health physics).

Radiation oncology, also known as radiotherapy, is one of the main methods used in treatment of cancer. It involves using ionizing radiations to kill tumour cells produced by malignant diseases. Radiation is normally delivered in such a way that a precisely defined tumour volume receives the maximum possible dose, while a minimal dose is delivered to the healthy tissue surrounding the tumour. The $\operatorname{dos}^{8}$ is a measure of the energy absorbed per mass of irradiated medium in units of Grays (Gy), where $1 \mathrm{~Gy}=1 \mathrm{~J} / \mathrm{kg}$.

In order to predict and assess the efficacy of the delivery of a radiotherapy treatment, quantitative measurements and calculations of the dose deposited by ionizing radiation into the body of the patient should be performed. Radiation dosimetry, the branch of medical physics that deals with measurement of dose, is based on an understanding of the nature of ionizing radiations used in radiotherapy as well as their interaction processes with tissues that lead to biological damage. This chapter introduces the basic principles of the production of clinical radio-therapeutic beams and radiation dosimetry. 


\section{Classification OF Radiations}

Radiation is classified into two main categories: ionizing and non-ionizing, depending on whether or not it is capable of ionizing matter through Coulomb interactions. Ionizing radiations can be further divided into two main groups: directly ionizing and indirectly ionizing ${ }^{9}$ as it is shown in Fig. 1-1. Directly ionizing radiations are represented by charged particles, such as electrons, positrons, protons, etc., which ionize atoms through direct Coulomb interactions with orbital atomic electrons. Indirectly ionizing radiations on the other hand, are neutral particles, such as photons and neutrons, which ionize matter through an intermediate step of first releasing a directly ionizing particle which then ionizes matter through Coulomb interactions. Photons release high energy electrons or positrons through various possible atomic interactions, such as the photoelectric effect, Compton effect, and pair production. The most widely used radiation types in radiotherapy are photons and electrons in the energy range from 1 to $25 \mathrm{MeV}$.

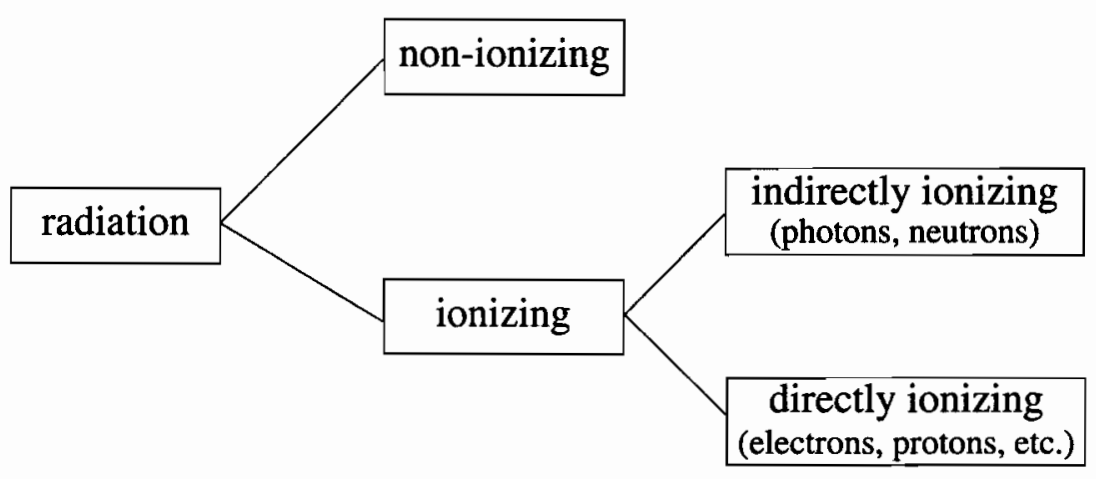

Figure 1-1. Classification of radiations as directly and indirectly ionizing radiations.

\section{DELIVERY OF RADIATION}

The most common radiation types used in radiotherapy are photons and electrons, although more exotic types such as protons and neutrons are also sometimes used for special radio-therapeutic procedures. The two modes of delivery of ionizing radiation are 
teletherapy with external radiation beams and brachytherapy with internally deposited sealed radioactive sources.

Teletherapy is the delivery of a collimated radiation beam whose source is a relatively large distance, typically $100 \mathrm{~cm}$, from the patient. The treatment machine usually is a medical linear accelerator (linac), see Fig. 1-2, or a cobalt-60 isotope machine (Co-60). The main components of a teletherapy treatment machine are a treatment couch on which the patient is positioned, and a gantry from which a collimated beam of ionizing radiation is emitted. The gantry and the couch both rotate around a fixed point (machine isocentre) to obtain the desired combination of collimated beams that compose a given treatment.

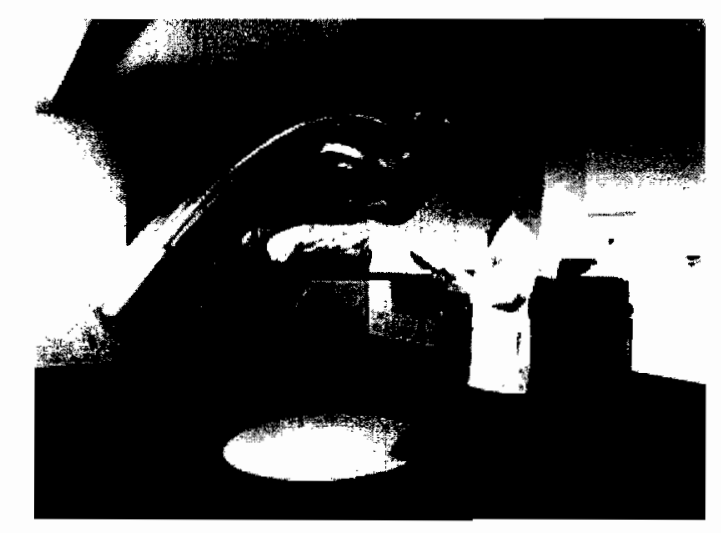

Figure 1-2. A medical linear accelerator used for external beam treatment.

Brachytherapy is the delivery of radiation from a short distance, i.e., immediately adjacent to or within the patient. It uses sealed radioactive sources to deliver radiation by interstitial, intracavitary, or surface application. With brachytherapy, a high dose can be delivered locally to a malignant tumor with rapid dose fall-off in the surrounding normal tissue. Afterloading devices are used to enable positioning of the sealed radioactive sources with remote control.

The sources of ionizing radiation emit either continuous or pulsed radiation.

Continuous radiation is produced by decaying radioisotopes or by low energy $\mathrm{x}$-ray machines which use a constant potential to accelerate a steady stream of electrons from a cathode to an anode (target) to produce continuous bremsstrahlung photons with $\sim 100 \mathrm{keV}$ energies. The source of continuous radiation that is the most widely used clinically is the cobalt-60 unit which employs ${ }^{60} \mathrm{Co}$ radionuclides that have a half life of 5.26 years, and decay to excited ${ }^{60} \mathrm{Ni}$ nuclei with the emission of $\beta$ particles $\left(E_{\max }=0.32 \mathrm{MeV}\right)$. The 
excited ${ }^{60} \mathrm{Ni}$ nuclei attain their ground states through an emission of two gamma photons per disintegration (1.17 and $1.33 \mathrm{MeV})$.

Pulsed radiation is produced by medical linear accelerators (linacs). High energy electrons strike the target as short pulses with a typical duration of $2 \mu$ s and a typical pulse repetition rate of 100 pulses per second. This relatively low duty cycle of $2 \times 10^{-4}$ protects the target from excessive heating and produces a clinical dose rate on the order of $300 \mathrm{cGy} / \mathrm{min}$ at the linac isocentre.

Continuous and pulsed radiations both ionize the medium in the same manner, however, they differ in the way that the created ions recombine. Ionic recombination is an effect that has to be taken into account when attempting to measure the absorbed dose within a medium using an air ionization chamber.

\section{TReATMENT PLANNING AND VERIFICATION}

In order to successfully use radiation to treat a malignant tumor while sparing healthy tissue, many parameters must be considered: these include determining the most appropriate type of radiation, the correct dosage, the size, shape and number of radiation fields, and their geometric location with respect to the tumor. Treatment planning refers to the process by which all of these parameters are accounted for and treatment planning software aids in accomplishing this task. In general, the 3 dimensional (3-D) geometry of the patient is obtained from a computerized tomography (CT) scan composed of a series of transverse images of the treated region of the patient. Important structures and the external contours of the patient are delineated on each individual slice (2-D transversal planes) to allow subsequent reconstruction of the structures in 3-D. The treatment planning software is used to calculate the dose given at every point within the patient volume from a given combination of beams. The best arrangement of beams (number, energy, incidence angle, size, beam modifiers, etc.) for a given treatment is found by optimizing the calculated dose distribution with the prescribed criteria from the physician's prescription. Most treatment planning softwares require measured beam data and semi-empirical formulae to calculate the total dose (sum of the contribution of all beams) everywhere within the patient volume. 
For external beams, two important measured parameters are used by the treatment planning software: the percent depth dose (PDD) and the off-axis ratios (OAR). Both parameters are measured as a function of beam field size and beam energy and for the different beam modifiers, such as wedge filters that are supplied with the linacs.

Depth dose curves illustrate the variation of absorbed dose with depth along the central beam axis within a patient or phantom. Two examples of $P D D$ curves are shown in Fig. 1-3 for a megavoltage photon (A) and a megavoltage electron (B) beam, respectively. Central axis depth doses are characterized by normalizing the dose to $100 \%$ at a reference depth, which is usually taken to be $d_{\max }$ or $R_{100}$, the depth at which the dose is maximum.

(A)

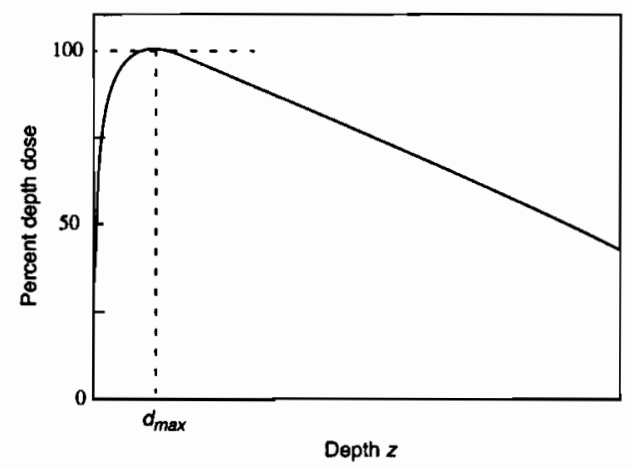

(B)

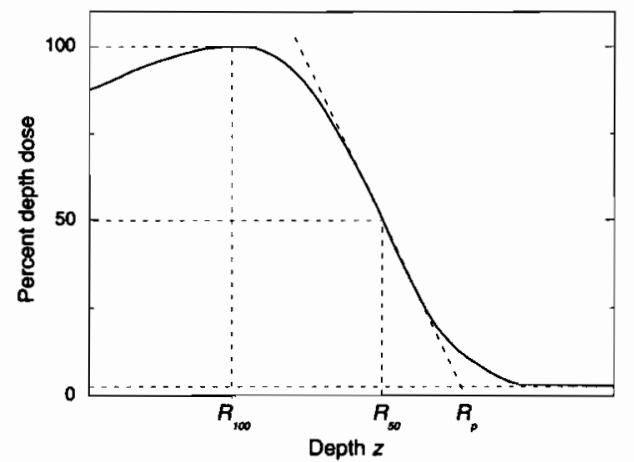

Figure 1-3. (A) Typical photon depth dose curve. (B) Typical electron depth dose curve. Both curves are normalized to a $100 \%$ at $d_{\max }$

The $O A R$ curves are perpendicular dose profiles of the beams obtained at standard depths in the phantom. An example of a collection of $O A R$ profiles for a $10 \times 10 \mathrm{~cm}^{2}$ $18 \mathrm{MV}$ photon beam at different depths is shown in Fig. 1-4.

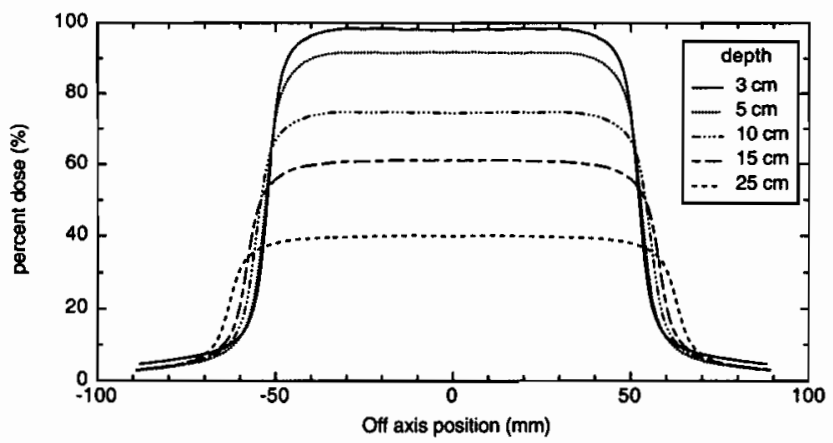

Figure 1-4. Typical off-axis ratio curves for various depths $\left(3 \mathrm{~cm}\left(d_{\max }\right), 5 \mathrm{~cm}, 10 \mathrm{~cm}, 15 \mathrm{~cm}, 25 \mathrm{~cm}\right)$ for a $18 \mathrm{MV}$ photon beam with $10 \times 10 \mathrm{~cm}^{2}$ field size. 
The standard conditions for obtaining $P D D$ s, $O A R$ s and isodose distributions are as follows: a homogeneous unit density cube-shaped phantom is fixed with its flat surface perpendicular to the beam axis at a known distance from the source. The data obtained from this irradiated set-up does not truly model what happens in the human body, but it is accurate to a first approximation when used as reference data ${ }^{10}$. So, in addition to the information obtained from these distributions, patient information has to be factored in to ensure accurate dose calculation with the treatment planning system. Patient contours and tissue electron densities are obtained through computerized tomography (CT). Using the given information about patient tissue inhomogeneities and body contours, a number of corrective algorithms are employed to modify the reference dose distributions. These algorithms correct for beam attenuation, scattering, oblique incidence of the beams and changes in source to surface distance (SSD). These algorithms produce relatively precise results; however, they suffer numerous limitations, one of the most important being the treatment of heterogeneities within the patient volume.

Algorithms have been developed to model the beams using convolution techniques or pencil beam techniques ${ }^{11,12}$. Some models work better than others depending on the particular geometry, but none of these algorithms are perfect because they all use some level of approximation. In particular, the dose in heterogeneous materials in the vicinity of an the interface between two different media is often poorly approximated. Monte Carlo calculations, see Chapter 5 , are much more adequate to model precisely the stochastic nature of radiation deposition and have been historically used as a research tool to verify and develop new algorithms. However, their clinical usefulness was limited by the relatively long times of computation. Constant advances in computer technology in recent years yielded a constantly decreasing ratio in "cost/computer power" now allowing the use of Monte Carlo techniques for routine treatment planning. ${ }^{13-16}$

Other important aspects of radiotherapy treatments are quality assurance, verification of treatments, and the calibration of radiation beams. Many techniques have been developed to measure the energy deposited by radiation. Often, a substitute for the human body is used, which is known as a phantom. An important criterion for a phantom is that it should be composed of materials that will absorb and scatter photons in the same way as human tissues ${ }^{17}$. The phantom material that is most commonly used is water because it 
radiologically behaves in almost exactly the same manner as soft tissue, which is composed of $75-90 \%$ of water ${ }^{18}$.

The parameters of clinical beams are measured with special devices referred to as dosimeters. The most commonly used dosimeters are ionization chambers. Standard ionization chambers require various calibration factors that are only available for water-equivalent phantoms. However, there are many clinical situations where a direct measurement of dose in materials different from water could be appealing. With the advent of clinical Monte Carlo calculations, the treatment of heterogeneities within the human body will now be considered in calculating the dose distributions. Most current treatment planning systems use numerous algorithms for approximations of dose corrections for the presence of heterogeneous materials within the human body. Because of calculation uncertainties most dose studies and protocols dealing with treatment planning, for example, the Radiation Therapy Oncology Group (RTOG), do not recommend the use of heterogeneity algorithms and require the calculations to be performed in a homogeneous material to ensure a uniformity of collected data.

\section{RADIATION DOSIMETRY TECHNIQUES}

One of the most important aspects of radiation therapy is the calibration of the clinical beams. Proper calibration of the treatment beams ensures that the prescribed dose for the treatment plan is actually delivered to the patient. A review of the principal clinical calibration protocols is given in Chapter 4 . In this section, we are giving an overview of the various methods for measuring the absorbed dose which represents the energy absorbed per unit mass of a medium.

Dosimetry can be divided into two main categories: absolute and relative dosimetry. An absolute dosimeter can measure absorbed dose in a given volume of material with no calibration of the dosimeter in a known radiation field, while a relative dosimeter requires calibration of the detector in a known radiation field. In radiotherapy centres, any measurement of dose should be traceable to an absolute dose standard at a standards laboratory. This is often done by having a "local" standard dosimeter calibrated at a standards 
laboratory and then this calibrated dosimeter is used as a secondary standard for cross-calibration of other local dosimeters.

There are many relative dosimetry techniques, such as ionization chambers, solid-state detectors, thermoluminescent detectors, and film which are commonly used in radiation dosimetry. On the other hands, there are only three absolute dosimetry techniques recognized by standard laboratories, each technique having a different degree of absoluteness. They are, from the most absolute to the least: calorimetry, chemical dosimetry, and standard free-air ionization chamber dosimetry.

\section{IV.A. Calorimetry}

Calorimeters are the most fundamental devices for absolute dosimetry, since they measure the absorbed dose directly. The energy absorbed by a medium from ionizing radiation produces heat energy and also a small amount of chemical change. A careful measurement of the temperature increase of the medium can be related to the energy absorbed per unit mass of the medium (absorbed dose). Small and sensitive devices, such as thermocouples and thermistors, are used to measure the change in temperature. Thermistors used in radiation dosimetry have a temperature-dependent resistance (resistance typically changes by $5 \%$ per degree) and have resistances on the order of $10^{3}$ to $10^{5} \mathrm{ohms}$. The small change induced in the resistance of the thermistor by the change of temperature can be measured very precisely with a Wheatstone bridge.

The energy absorbed can be related to the temperature by the thermal-capacity (specific heat $c_{p}$ ) of the medium expressed in $\mathrm{J} / \mathrm{kg}{ }^{\circ} \mathrm{C}$ and by the thermal defect $\delta_{T}$, which quantifies the amount of energy that does not appear as heat, through the following equation:

$$
\Delta T=\frac{E}{m} \frac{\left(1-\delta_{T}\right)}{c_{p}}=D \frac{\left(1-\delta_{T}\right)}{c_{p}}
$$


where $\Delta T$ is the change of temperature of the medium, $E$ the energy deposited by radiation in the sensitive volume of mass $m$, and $D$ the absorbed dose to the medium. Therefore the absorbed dose to the medium is given by:

$$
D=\frac{\Delta T c_{p}}{\left(1-\delta_{T}\right)}
$$

Since the energy imparted to a medium by radiation is very small, calorimeters must be extremely sensitive. They are bulky, complex to operate, and measurements are lengthy, since it requires a long period of time to reach thermal stability. Their use is therefore restricted to standards laboratories and they are impractical for routine clinical dosimetry.

\section{IV.B. Chemical dosimetry}

In chemical dosimetry, absorbed dose is determined from a quantitative measurement of a chemical change in a given medium exposed to radiation. In general, chemical dosimeters are water-based liquid solutions for the measurement to be performed in a tissue equivalent material. The most commonly used chemical dosimeter is the Fricke dosimeter which uses an aqueous ferrous sulfate solution ${ }^{10}$.

In aqueous solutions, radiation will first interact with water to produce chemically active species, such as free radicals and ion pairs near the particle tracks. After $\sim 10^{-6} \mathrm{~s}$ the distribution of the primary products will become homogeneous within the irradiated solution volume by the diffusion process. The primary products will chemically react with active species of the dosimeter and a final chemical product $X$ will be produced.

The number ${ }^{19}$ of molecules of the product $X$, destroyed or changed by $100 \mathrm{eV}$ of radiation energy is called the radiation chemical yield $G(X)$ in molecules/100 eV, or in mol/J by multiplying the $G(X)$ value by $1.037 \times 10^{-7}$. The absorbed dose $D$ found by measuring the quantity of $X$ produced during the irradiation is given by:

$$
D=\frac{\Delta M}{\rho G(X)}
$$


where $\Delta M(\mathrm{~mol} / \mathrm{l})$ is the change of molar concentration of $X, \rho$ is the solution density, nor-

mally close to water density $\left(1 \mathrm{~g} / \mathrm{cm}^{3}\right)$, and $G(X)$ is normally assumed to be constant during the irradiation.

The Fricke solution contains ferrous $\mathrm{Fe}^{2+}$ ions that are converted to ferric $\mathrm{Fe}^{3+}$ ions by chemical reactions induced by radiation and is suitable ${ }^{19}$ to measure dose in the range from $4 \mathrm{~Gy}$ to $400 \mathrm{~Gy}$. The most efficient method to measure the change of molar concentration $(\Delta M)$ of $\mathrm{Fe}^{3++}$ is spectroscopy requiring a small sample of the solution $(\sim 1 \mathrm{~cm}$ pathlength) for which the transmission of laser light (304 nm) is measured.

Chemical dosimeters have the advantage of being mainly composed of water and can be considered tissue-equivalent. However, the solutions used for chemical dosimeters are expensive, difficult to prepare, and often toxic. Moreover, $G(X)$ is sensitive to temperature during the irradiation and spectroscopy readout phase, and is not known to better than a $\pm 2 \%$ accuracy. The dosimeters are also very sensitive to impurities in the ferrous sulfate solutions. All these disadvantages preclude the use of Fricke dosimeters for routine clinical radiation dosimetry.

\section{IV.C. Free-air ionization chamber}

The absorbed dose can be determined from a measurement of the exposure in a standard free-air ionization chamber. Exposure $X(\mathrm{C} / \mathrm{kg})$ is defined as the total charge of one sign produced in dry air when all secondary electrons liberated by photon interactions in a unit mass of air are completely stopped in air $^{8}$. It is expressed as:

$$
X=\frac{d Q}{d m}
$$

where $d Q$ is the charge and $d m$ is the mass element of air.

The old unit of exposure is the Roentgen $\left(1 \mathrm{R}=2.5810^{4} \mathrm{C} / \mathrm{kg}\right)$. Exposure refers to all electrons set in motion by photons in a given volume of air with mass $m$. The ion pairs that are created are collected from throughout the electron's path as the electron slows down in air. Exposure is easily measured for low-energy photons $(<300 \mathrm{keV})$, however, for 
megavoltage energies the range of secondary electrons in air becomes too large, making the required chamber dimensions cumbersome for use in practical dosimetry.

Once the exposure $X$ is known, the dose in air at the point of interest is expressed as:

$$
D_{\text {air }}=X \cdot \bar{W}_{\text {air }}
$$

where $\bar{W}_{a i r}$ is the mean energy required to produce an ion pair in air. $\bar{W}_{a i r}$, expressed in eV/ion pair, is an important quantity for calibration protocols. As an electron slows down in an air cavity, it loses energy by ionizing the gas and hence producing ions. Measuring the number of ions created in the ionization chamber cavity is the basic measurement used to obtain the kinetic energy released in matter (kerma) and eventually the absorbed dose.

For dry air and for electron energies larger than a few $\mathrm{keV}, \bar{W}_{\text {air }}$ is assumed to be a constant. It is more useful to normalize this quantity in terms of $\mathrm{J} / \mathrm{C}$, i.e., dividing by the charge of the electron to obtain $\bar{W}_{\text {air }}=(33.97 \pm 0.05) \mathrm{J} / \mathrm{C}$ which gives the energy deposited in air per Coulomb of charge released in dry air. This value is recommended by the ICRU report 37 (Ref. \#20) and was also verified by Boutillon and Perroche-Roux ${ }^{21}$. $\bar{W}_{\text {air }}$ depends on humidity, for example, at $50 \%$ humidity $\bar{W}_{\text {air }}$ decreases by $\sim 0.6 \%$ from its dry air value. It is generally assumed that ion chamber air cavities have the same humidity level as the ambient air. The mass of air in the cavity $m_{g a s}$ as well as the stopping power ratios $(\bar{L} / \rho)_{\text {med }_{2}}^{\text {med }}$ are also a function of the humidity of the cavity and this has caused some confusion in calibration protocols ${ }^{22}$. A constant humidity correction factor $K_{h}=0.997$, applicable over a large range of humidity from $15 \%$ to $80 \%$, is used by the latest AAPM calibration protocol (TG-51) (Ref. \#23). The humidity correction factor is normally included in the basic output calibration of clinical ionization chambers.

Exposure is derived from a measurement of the total ionization produced by energetic electrons arising from photon interactions with a known mass of air. The free-air ionization chamber is a device that can perform such a measurement. The free-air ionization chamber was designed by Attix and Wyckoff ${ }^{24}$ and has been used as a primary measurement standard for dosimetry protocols ${ }^{25}$. It consists of an open-air chamber which has two parallel plates with a potential across them for collecting the charge liberated by a well defined photon beam passing between them. 
A schematic representation of the free air ionization chamber is given in Fig. 1-5. A lead box with an aperture $A_{O}$ encloses a collecting electrode that defines the collecting volume $V$ for which the exposure is measured. Therefore all ionization produced by secondary electrons originating from $V$ should be collected in collecting volume $V$ ' to contribute to the measured exposure; examples of such electrons are illustrated by $\left(e_{2}\right)$ and $\left(e_{3}\right)$ in Fig. 1-5. However, some electrons created within volume $V$ will escape volume $V^{\prime}$ and some of the ions they produce will not be collected by the collecting electrode; those electrons are illustrated by $\left(e_{3}\right)$ in Fig. 1-5. Furthermore, some electrons created outside the volume $V^{\prime}$ may cross into $V^{\prime}\left(e_{1}\right)$ and produce ions that will contribute to the exposure. Therefore, the lateral dimensions of the chamber are chosen such that charged particle equilibrium (CPE) exists throughout the sensitive volume $V^{\prime}$. CPE is defined in detail in Section I.D of Chapter 4 and requires that only ionization produced by secondary electrons produced in the volume $V$ must be collected. In practice, this requirement is satisfied by the fact that the number of secondary electrons that are produced in $V$ and escape $V^{\prime}\left(e_{3}\right)$ is exactly balanced by electrons that are originating outside $V$ and enter $V^{\prime}\left(e_{1}\right)$.

The lateral dimensions of the chamber are chosen such that charged particle equilibrium exists throughout $V^{\prime}$. This is done by positioning all components of the chamber: the polarizing, collecting, and guard electrodes, as well as the walls of the lead box, far enough from the boundaries of $V$ so that no electrons produced in $V$ can reach them.

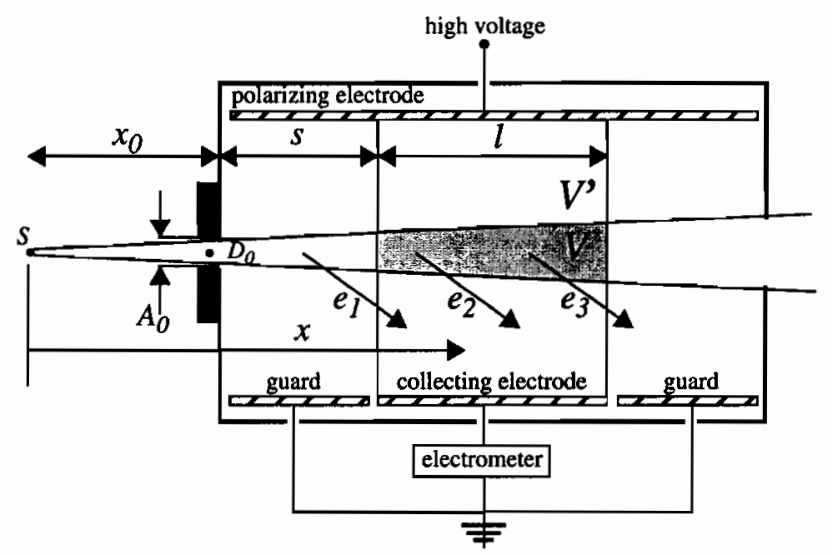

Figure 1-5. Schematic diagram of a free-air ionization chamber. The charge collecting volume is $V$ ' and the ideal volume is $V$ where secondary electron are produced for collection $\left(e_{2}\right)$. The secondary electrons produced in $V$ that escape the collection volume $V^{\prime}\left(e_{3}\right)$ are exactly balanced by the electrons produced outside $V$ that enter $V^{\prime}\left(e_{l}\right)$ for collection. 
The aperture area of the free-air ionization chamber is $A_{0}$ and is located at a distance $x_{0}$ from the radiation source $S$. A disc of air of thickness $d x$, located in the plane of the aperture, has a mass of $d m_{0}=\rho A_{0} d x$. An incident photon beam with energy fluence $\psi_{0}$ is crossing the mass element $d m_{0}$ that contains the point $D_{0}$ creating electrons through interactions within $d m_{0}$. The electrons set in motion will dissipate their kinetic energy in air and produce charges of either sign equal to:

$$
d Q_{0}=\frac{\psi_{0}}{\bar{W}_{a i r}}\left(\frac{\mu_{a b}}{\rho}\right)_{a i r} d m_{0}=K_{0} d m_{0}=K_{0} \rho A_{0} d x
$$

where $\left(\mu_{a b} / \rho\right)_{\text {air }}$ is the mass energy absorption coefficient for air defined in Chapter 3, Section IV.H and $K_{0}=\left(\psi_{0} / \bar{W}_{a i r}\right)\left(\mu_{a b} / \rho\right)_{a i r}$ is the kerma in air. A mass element of air $d m$ positioned at a distance $x$ from the source and lying inside the volume $V$ has a mass given by:

$$
d m=\rho A d x=\rho A_{0}\left(\frac{x}{x_{0}}\right)^{2} d x
$$

The photon energy fluence at that distance $x, \psi(x)$, is found from the fluence at the aperture $x_{0}$ by the inverse-square law and the exponential attenuation of the beam:

$$
\psi(\mathrm{x})=\psi_{0}\left(\frac{x_{0}}{x}\right)^{2} \mathrm{e}^{-\mu\left(x-x_{0}\right)}
$$

where $\mu$ is the linear attenuation coefficient in air of the beam defined in Chapter 2 . The charge $d Q$ produced by secondary electrons originating in $d m$ is:

$$
d Q=\frac{\psi(x)}{\bar{W}_{\text {air }}}\left(\frac{\mu_{a b}}{\rho}\right)_{\text {air }} d m=K_{0} e^{-\mu\left(x-x_{0}\right)} \rho A_{0} d x
$$

If $s$ is the distance from the aperture to the collecting electrode and $l$ is the width of the collecting volume $V^{\prime}$, integrating Eq. (1-9) between $\left(x_{0}+s\right)$ and $\left(x_{0}+s+l\right)$ yields the total charge collected $Q$ : 


$$
Q=K_{0} \rho A e^{-\mu x_{0}} \int_{x_{0}+s}^{x_{0}+s+1} e^{-\mu x} d x=K_{0} \rho A\left(\frac{e^{-\mu s}}{\mu}\right)\left[1-e^{-\mu l}\right]
$$

expanding the $\left[1-\mathrm{e}^{-\mu l}\right]$ term yields:

$$
Q \approx K_{o} \rho A\left(\frac{e^{-\mu s}}{\mu}\right)[1-(1-\mu l)]=K_{0} \rho A l e^{-\mu s}
$$

If we consider a cylindrical volume $A_{0} l$ of mass $M_{c}=\rho A_{0} l$, from Eq. (1-11) we can deduce $Q / M_{c}=K_{0} e^{-\mu s}$ and, from Eq. (1-6), the exposure $X_{0}=d Q_{0} / d m_{0}$ at point $D_{0}$ is found from the measured exposure $Q / M_{c}$ using:

$$
X_{0}=\frac{d Q_{0}}{d m_{0}}=\frac{Q}{M_{c}} e^{\mu s}
$$

Therefore, the exposure at point $D_{0}$ is the same as the exposure that is measured in the free-air ionization chamber corrected for the attenuation of the photon beam in air. The free-air ionization chamber will be used to calibrate the readings of secondary standard cavity chambers located at point $D_{0}$.

This principle works very well for low-energy photons, and allows a direct measurement with an accuracy of about $\pm 0.5 \%$. The measurement technique breaks down for higher photon energies, since the size of the chamber becomes a concern. For example, electrons set in motion by a monoenergetic photon beam of $3 \mathrm{MeV}$ have a range of $1.5 \mathrm{~m}$ in air. Therefore the total length of the guard and collecting electrodes should be over $3 \mathrm{~m}$ and an air gap of $1.5 \mathrm{~m}$ will attenuate the beam by more than $5 \%$. For these reasons it is impractical to use a free-air chamber at photon energies above $3 \mathrm{MeV}$.

At these high energies very different techniques using ionization chambers and Bragg-Gray cavity theory are used to measure exposure (as discussed in Chapter 4). However, the use of exposure has two inherent problems. The first is that exposure is only defined for photons interacting in air; the second is that the quantity becomes ill-defined as photon energies become higher as in medical linac megavoltage beams because the range of the electrons slowing down in air becomes excessively large. 
These problems are solved by introducing the kerma (discussed in Chapter 4), which is the Kinetic Energy Released in Medium (unit: $\mathrm{J} / \mathrm{kg}$ or gray). For photon beams the kinetic energy released is the kinetic energy transferred to electrons in the material. The quantity is defined with respect to a specific material in which interactions are taking place (e.g., air-kerma, water-kerma). This quantity is well defined at all energies and for all materials. It is expressed as the sum of all energy transfers to charged particles in the volume divided by the mass of the volume. Air-kerma and exposure are closely related, however, air-kerma is not directly measurable. Kerma plays an important role in radiation dosimetry because it is the energy released per unit mass of material and this is closely related to the energy absorbed per unit mass of material.

\section{IV.D. Phantom-embedded extrapolation chamber (PEEC)}

The use of a Solid Water ${ }^{\mathrm{TM}}$ phantom-embedded extrapolation chamber (PEEC) was proposed by Zankowski and Podgorsak ${ }^{26,27}$ as an absolute dosimeter with equal absoluteness to the free-air ionization chamber. In the work reported here we have evaluated the use of a modified extrapolation chamber designed to directly measure absolute dose in heterogeneous materials. The PEEC has several advantages: the dosimeter is simple to use, is based on first principles and yields a direct dose measurement in comparison to relative dosimetry techniques.

Determination of the absorbed dose in megavoltage photon and electron beams with ionization chambers requires the use of Bragg-Gray and Spencer-Attix cavity theories. The dose is related to the ratio $(Q / m)$, where $Q$ is the collected charge and $m$ is the mass of air of the cavity of the chamber. $Q$ can be easily measured to a high degree of accuracy, but $m$ is very difficult to determine. This is why clinical chambers have to be calibrated at a standards laboratory or at least cross-calibrated with a local calibrated dosimeter. The calibration factor of a given chamber is influenced by the dimensions and composition of the chamber as well as by the radiation type and energy. 
Spencer-Attix theory (as discussed in Chapter 4), relates the dose to the medium $D_{m e d}$ to the measured charge $Q$ by:

$$
D_{m e d}=\frac{Q}{m} \bar{W}_{a i r}\left(\frac{\bar{L}}{\rho}\right)_{\text {air }}^{m e d}
$$

where is $(\bar{L} / \rho)_{\text {air }}^{\text {med }}$ is the ratio of the mean restricted stopping powers in the medium and in air.

The phantom-embedded extrapolation chamber is a variable-volume parallel-plate extrapolation chamber embedded directly into Solid Water ${ }^{\mathrm{TM}}$ (water-equivalent material). This chamber can be used to determine the derivative $d Q / d m$ through a measurement of the change in charge $d Q$ as a function of a change of mass $d m$ of the sensitive volume of the chamber. The Spencer-Attix cavity theory equation Eq. (1-13) can be modified as follows:

$$
D_{m e d}=\frac{d Q}{\rho A d z} \bar{W}_{a i r}\left(\frac{\bar{L}}{\rho}\right)_{a i r}^{m e d} \text {, }
$$

where the $Q / m$ ratio was replaced by $d Q / \rho A d z$ with $\rho$ the density of air $^{28}, A$ the effective area of the collecting electrode, and $d z$ the relative displacement of the electrodes such that, $\rho A d z=d m$. $A$ can also be determined through a simple capacitance measurement with the chamber. Therefore the PEEC is an uncalibrated air-ionization chamber which can determine the absorbed dose to a level of absoluteness similar to a free-air ionization chamber but is not restricted to beam energies less than $3 \mathrm{MeV}$. It also yields an in-phantom dose directly, and obviates the use of various correction factors, such as the ones, as discussed in Chapter 4, used by national and international dosimetry protocols.

\section{MOTIVATION FOR THIS WORK}

In this work, we are evaluating the use of the phantom-embedded extrapolation chamber to measure directly the absorbed dose in heterogeneous materials. As we mentioned earlier the recent popularity of algorithms based on Monte Carlo techniques will 
require measurement verification techniques for quality assurance of treatments. PEEC offers a relatively simple way of determining an absolute absorbed dose at a point in phantom without the use of many cumbersome calibration factors of the clinical protocols that moreover lack definitions for materials that are not water-equivalent. A modular design was studied so that the PEEC can be used as a versatile tool to perform this task.

The characteristics of the PEEC when operated for dose measurements in bone material, were verified. Monte Carlo modelling is carried out to compare measurement in different situations, namely to study the effect of the particular geometry of the PEEC phantom, PEEC electrodes and cavity.

\section{SUMMARY}

Radiation therapy is an important modality for treatment of malignant disease. Ionizing radiation is either directly (charged radiation) or indirectly (uncharged radiation) ionizing. In a biological system, radiations ionize and excite water molecules which in turn, through chemical reactions, produce other ions and free radicals which are capable of causing large scale biological damage by killing cells.

A patient treatment in a radiotherapy clinic has first to be planned by treatment planning software. These softwares use various measured beam data and dose calculation algorithms with a goal of optimizing the treatment parameters thereby maximizing the dose to the target volume and minimizing the dose to healthy tissues surrounding the tumours.

Calibration and verification of treatment machines that deliver radiation to the patient are of primary importance. Several techniques exist to measure the absorbed dose delivered from a radiation beam. Dosimetric measurements are divided into absolute and relative measurements. Absolute measurement techniques do not require a calibration of the dosimeter in a known radiation field while relative dosimeters must be calibrated in a known radiation field. Calorimetry, chemical dosimetry and standard free-air ionization chamber dosimetry represent the three absolute dosimetry techniques. Because of their complexity, these techniques are only available at standards laboratories. Radiotherapy clinics have to send one of their relative dosimeters to standards laboratories to obtain a cal- 
ibration factor that is applicable in restricted condition, namely only for calculating the absorbed dose to a water phantom. In this work we studied the use of a phantom-embedded extrapolation chamber for direct measurement of dose in heterogeneous phantoms. This approach provides the only reliable measurement technique for verification of dose distributions in heterogeneous phantoms calculated by Monte Carlo techniques. 


\section{References}

1 Otto Glasser, Jessie C. Tucker, and Margret Boveri, Wilhelm Conrad Röntgen and the early history of the Roentgen rays (Bale \& Danielsson, London, 1933).

2 Otto Glasser and Wilhelm Conrad Röntgen, Dr. W. C. Röntgen (C. C. Thomas, Springfield, 1ll., 1945).

3 Wilhelm Conrad Röntgen, Adrian Thomas, Ian Isherwood et al., The Invisible Light : 100 years of medical radiology (Blackwell Science Ltd, Oxford; Cambridge, Mass., 1995).

4 Francis H. Williams, The Roentgen rays in medicine and surgery: as an aid in diagnosis and as a therapeutic agent designed for the use of practitioners and students (Macmillan, New York, 1901).

5 M. D. Blaufox, "Becquerel and the discovery of radioactivity: early concepts," Semin. Nucl. Med. 26 (3), 145-154 (1996).

6 J. Dutreix and A. Dutreix, "Henri Becquerel (1852-1908)," Med. Phys. 22 (11 Pt 2), 1869-1875 (1995).

7 J. Dutreix, "From X-rays to radioactivity and radium. The discovery and works of Henri Becquerel (1851-1908)," Bull. Acad. Natl. Med. 180 (1), 109-118 (1996).

8 ICRU, "Radiation quantities and units," ICRU Report 33, International Commission on Radiation Units and Measurements, Washington, D.C. (1980).

9 ICRU, "Radiation quantities and units," ICRU Report 19, International Commission on Radiation Units and Measurements, Bethesda, Maryland (1971).

10 Faiz M. Khan, The physics of radiation therapy, 2nd ed. (Williams \& Wilkins, Baltimore, 1994). 
11 T.R. Mackie, P. Reckwerdt, T. McNutt et al., "Photon beam dose computations," in Teletherapy Physics, Present and Future, edited by J.R. Palta and T.R. Mackie (Advanced Medical Publishing, Madison, WI, 1996), pp. 103-174.

12 J. Van Dyk, R.B. Barnett, and J.J. Battista, "Computerized Radiation Treatment Planning Systems," in The modern technology of radiation oncology: a compendium for medical physicists and radiation oncologists, edited by J. Van Dyk (Medical Physics Pub., Madison, Wis., 1999), pp. 231-286.

13 NOMOS Corporation, PEREGRINE (NOMOS Corporation, Sewickley, PA, 2000).

14 I. Kawrakow, M. Fippel, and K. Friedrich, “3D electron dose calculation using a Voxel based Monte Carlo algorithm (VMC)," Med. Phys. 23 (4), 445-457 (1996).

15 C. M. Ma, E. Mok, A. Kapur et al., "Clinical implementation of a Monte Carlo treatment planning system,” Med. Phys. 26 (10), 2133-2143 (1999).

16 K. Weaver, C. H. Siantar, W. Chandler et al., "A source model for efficient brachytherapy computations with Monte Carlo," Med. Phys. 23 (12), 2079-2084 (1996).

17 Harold Elford Johns and John Robert Cunningham, The physics of radiology, 4th ed. (Charles C. Thomas, Springfield, Ill., U.S.A., 1983).

18 J. E. Turner, Atoms, radiation, and radiation protection, 2nd ed. (J. Wiley, New York, 1995).

19 Frank H. Attix, Introduction to radiological physics and radiation dosimetry (Wiley \& Son, New York, 1986).

20 ICRU, "Stopping powers for electrons and positrons," ICRU Report 37, International Commission on Radiation Units and Measurements, Bethesda, Maryland (1984).

21 M. Boutillon and A. M. Perroche-Roux, "Re-evaluation of the W value for electrons in dry air," Phys. Med. Biol. 32, 213-219 (1987). 
22 D. W. O. Rogers, "Fundamentals of dosimetry based on absorbed-dose standards," in Teletherapy Physics, Present and Future, edited by J.R. Palta and T.R. Mackie (Advanced Medical Publishing, 1996), pp. 319-356.

23 P. R. Almond, P. J. Biggs, B. M. Coursey et al., “AAPM's TG-51 protocol for clinical reference dosimetry of high-energy photon and electron beams," Med. Phys. 26 (9), 1847-1870 (1999).

24 H. Wyckoff and F. H. Attix, "Design of free-air ionization chambers," Handbook 64, National Bureau of Standards, Washington, D.C., (1957).

25 AAPM Task Group 21 of the Radiation Therapy Committee, "A protocol for the determination of absorbed dose from high-energy photon and electron beams," Med. Phys. 10, 741-771 (1983).

26 C. E. Zankowski, "Calibration of photon and electron beams with an extrapolation chamber," Ph.D. Thesis, McGill University, 1997.

27 C. E. Zankowski and E. B. Podgorsak, "Calibration of photon and electron beams with an extrapolation chamber," Med. Phys. 24 (4), 497-503 (1997).

28 Chemical Rubber Company, CRC Handbook of Chemistry and Physics, 78th ed. (Chemical Rubber Pub. Co., Cleveland, 1997). 


\section{Chapter 2}

\section{Interactions of photons with matter}

\section{INTRODUCTION}

\section{I.A. Types of indirectly ionizing photon radiations}

Photons are indirectly ionizing radiations, since they can only ionize matter through an intermediate step of first releasing or producing directly ionizing particles (primarily electrons and positrons). These particles then ionize matter through Coulomb interactions with orbital electrons of absorber atoms. This chapter introduces the various processes by which photons interact with matter to produce the directly ionizing particles. The Coulomb interactions of the directly ionizing particles are discussed in Chapter 3.

Depending on their origin, the indirectly ionizing photon radiations fall into one of the following four categories:

1. Bremsstrahlung (continuous $\mathrm{x}$ rays) emitted through energetic electron-nucleus interactions.

2. Characteristic $x$ rays (discrete) emitted in transitions of atomic orbital electrons from one allowed orbit to a vacancy in another allowed orbit.

3. Gamma rays (discrete) emitted through nuclear transitions in gamma decay.

4. Annihilation radiation (discrete, typically: $0.511 \mathrm{MeV}$ ) emitted through positron annihilation with an orbital electron. 


\section{I.B. Photon beam attenuation}

As they pass through an attenuating material, photon beams undergo an intensity diminution rather than an energy change. The intensity $I(x)$ of a narrow monoenergetic photon beam, attenuated by an attenuator of thickness $x$, is governed by:

$$
I(x)=I(0) \mathrm{e}^{-\mu x},
$$

where $I(0)$ is the original intensity of the unattenuated beam and $\mu$ is the linear attenuation coefficient depending on photon energy $h v$ and atomic number $Z$ of the attenuator. Attenuation of photon beams which possess a spectral distribution is more complicated because of the dependence of $\mu$ on photon energy. This dependence then causes changes in photon spectrum with thickness of attenuator traversed.

\section{I.C. Types of photon interactions}

Photons may undergo various possible interactions with atoms of an attenuator; the probability or cross-section for each interaction depends on energy $h v$ of the photon and atomic number $Z$ of the attenuator. The photon interactions may be with an atom as a whole (photoeffect), with the field of the nucleus (pair production), or with orbital electrons (coherent scattering, Compton effect, triplet production). The photon-orbital electron interaction may be with an essentially free orbital electron (Compton effect) or with a tightly bound orbital electron (coherent scattering, photoeffect). In this context, a tightly bound electron is defined as an electron with a binding energy comparable to, but smaller than the incident photon energy; i.e., $E_{b} \leqslant h v$. A loosely bound or free electron, on the other hand, is an orbital electron with a binding energy much smaller or negligible in comparison with the incident photon energy; i.e., $E_{b}<h v$.

During the interaction the photon may completely disappear (photoeffect, pair production, triplet production) or it may be scattered coherently (coherent scattering) or incoherently (Compton effect). 
Photon interactions with matter are generally described through the kinematic and probabilistic aspects of the various possible interactions. The kinematic aspects of the interactions are described in terms of the energy transferred and the angles between the incident photon and scattered particles for a given process. The probabilistic aspects of the photon interactions, on the other hand, are described through interaction cross-sections which represent the likelihood that a particular reaction mechanism will take place as a function of incident photon energy and the atomic number of the medium with which the photon interacts.

\section{KinEMATICS OF PHOTON INTERACTIONS}

Photon interactions with matter are governed by a probability of interaction per unit distance travelled. When an interaction takes place, the photon might be absorbed and disappear from the primary beam or it might be scattered, changing its direction of travel with or without a loss of energy.

The mechanisms by which the photons interact with matter without an appreciable transfer of energy are Rayleigh (coherent) and Thompson scattering. Rayleigh scattering is a coherent scattering in which the photon is elastically scattered by the combined action of the whole atom. The photon, scattered at a small average angle, loses essentially none of its energy, while the atom moves to conserve momentum. In Thompson scattering a free electron will oscillate classically when absorbing the energy of the passing photon. The oscillating electron will readily re-emit photons of the same frequency as the incident wave. The interaction is elastic and only causes a redirection of the incident photon. Thompson scattering is the low-energy limit of Compton scattering with the incident photon energy approaching zero.

Principal mechanisms of photon interactions with matter in which an appreciable amount of energy may be transferred to charged particles are Compton scattering, pair production, and photoeffect. Because of their importance in radiation dosimetry we are treating these processes separately in some detail below. 


\section{II.A. Photoelectric effect}

The photoelectric effect is the process by which an orbital electron is ejected as a result of an atom absorbing a photon, as illustrated schematically in Figure 2-1. In this process practically all the initial energy $h v$ of the photon is transferred to the atomic electron and only a small fraction of the incident photon energy $\left(\sim 10^{-5}\right)$ is absorbed by the atom. The kinetic energy $\left(T_{e-}\right)$ of the emitted photoelectron is equal to the photon energy $h v$ minus an energy $\psi$ that the electron expends in escaping the atom, i.e., $T_{e^{-}}=h v-\psi$. The minimum quantity $\psi_{o}$, called the work function of the material, is equal to the minimum binding energy of an electron in this atom $-\left|E_{b}\right|_{\min }$. In general $T_{e^{-}}=h v-\left|E_{b}\right|$, since interactions of this type can occur with electrons from any of the electron shells. The photoelectric interaction is most probable when the photon energy $h v$ is only slightly larger than the electron binding energy in the particular atomic shell.

The angle at which the photoelectron is ejected is related to the energy of the incoming photon. Therefore, the photoelectric interaction is said to be an interaction of a photon with a tightly bound electron.

At low photon energies ( $h v \leq 20 \mathrm{keV}$ ) the photoelectrons are ejected primarily at right angles to the direction of the incident photon, whereas for $h v \geq 1 \mathrm{MeV}$, they are emitted mostly in the forward direction ${ }^{1}$.

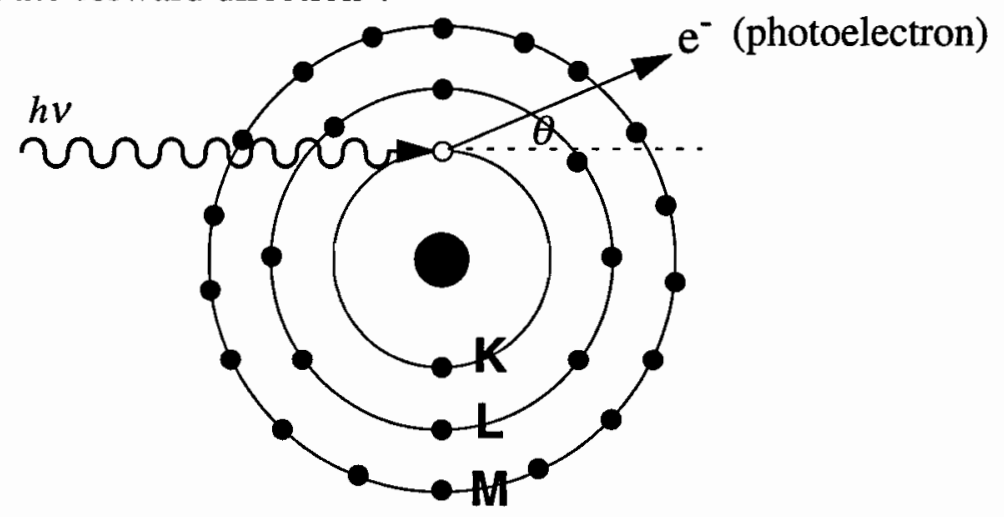

Figure 2-1. Schematic diagram of the photoelectric process. A photon with quantum energy $h v$ interacts with an atomic electron that has a binding energy $E_{b}$. The photon vanishes and the electron is ejected from the atom with a kinetic energy $T_{e}=h v-\left|E_{b}\right|$ at an angle $\theta$ relative to the incident photon's direction. The atom departs at an angle $\varphi$ in order to conserve momentum. The kinetic energy $T_{a}$ transferred to the above is practically zero because of the relatively large mass of the atom in comparison with the mass of the photoelectron. 
After the ejection of the atomic electron, the atom is left in an excited state with an electron vacancy in one of the electron shells. The excess atomic energy will be released by one of several possible processes that are discussed in Section III below. The initial vacancy in the shell will move to vacancies in higher shells, which in turn will eventually be filled by the capture of thermal electrons returning the atom back to its original neutral state.

\section{II.B. Compton effect (incoherent scattering)}

The Compton interaction is produced by a photon of energy $h v$ interacting with an essentially free and stationary electron. The electron is considered free as long as the energy of the photon is much greater than the binding energy of the electron in a shell ${ }^{2}$. The electron receives some energy from the photon and is ejected at an angle $\varphi$, while the resulting less energetic photon will be scattered at an angle $\theta$. Similarly to photoeffect, the Compton process leaves the atom with an electron vacancy in one of its atomic shells. This vacancy is eventually filled by one of the processes described in Section III. The Compton process is illustrated schematically in Fig. 2-2.

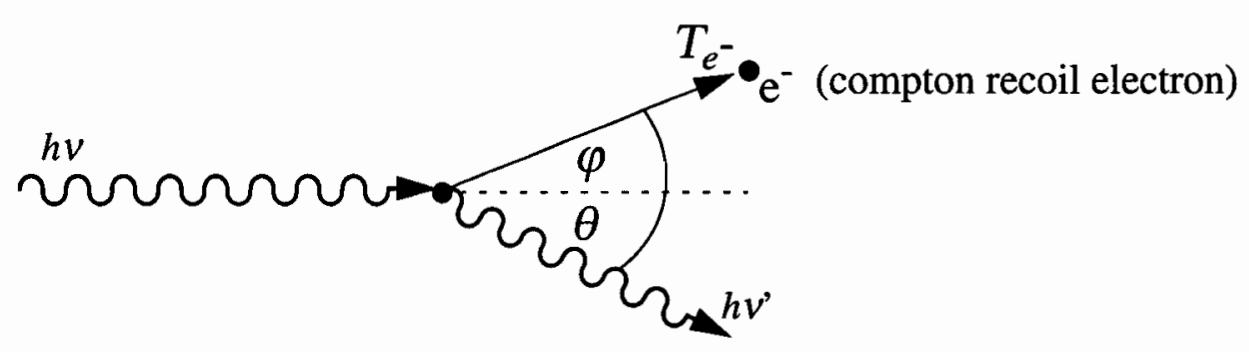

Figure 2-2. Schematic diagram of the Compton process. An incident photon with energy $h v$ interacts with a stationary and free electron. The photon is scattered at an angle $\theta$ with an energy $h v^{\prime}$ and the electron recoils at an angle $\varphi$ with a kinetic energy $T_{e^{-}}$. 
The collision process of a Compton interaction is described simply by applying the relativistic laws of conservation of momentum and energy to get:

$$
\begin{gathered}
T_{e^{-}}=h v \frac{\varepsilon(1-\cos \theta)}{1+\varepsilon(1-\cos \theta)}, \\
h v^{\prime}=\frac{h v}{1+\varepsilon(1-\cos \theta)}, \\
\lambda^{\prime}-\lambda=\frac{h c}{m_{0} c^{2}}(1-\cos \theta),
\end{gathered}
$$

and,

$$
\cot \varphi=(1+\varepsilon) \tan (\theta / 2)
$$

where $h v, h v^{\prime}$ and $T_{e^{-}}$are the energies of the incoming photon, scattered photon and recoil electron, respectively. The parameter $\varepsilon$ is given by $\varepsilon=h v / m_{0} c^{2}$ with $m_{\mathrm{o}} c^{2}=0.511$ $\mathrm{MeV}$, the rest mass energy of the electron, and $h$ the Planck's constant.

\section{II.C. Pair and Triplet production}

Pair production is an interaction process in which a photon disappears and an electron-positron pair $\left(\mathrm{e}^{+}, \mathrm{e}^{-}\right)$is created in the Coulomb field of a nucleus which recoils to conserve momentum. The process is illustrated schematically in Fig. 2-3. The threshold photon energy for pair production is the energy required to produce an electron-positron pair, i.e., $h v>2 m_{0} c^{2}=1.022 \mathrm{MeV}$. Because of the large nuclear mass, essentially no energy is transferred to the nucleus and the energy of the photon $h v$ is converted into electron and positron rest mass (1.022 MeV), while the remaining energy is distributed between the electron and positron kinetic energies $T_{e^{-}}$and $T_{e}+$, respectively, to conserve energy:

$$
T_{e^{-}}+T_{e^{+}}=h v-2 m_{0} c^{2}
$$


The pair production process can also occur in the Coulomb field of a single orbital electron; in this case the interaction yields three particles; the $\left(\mathrm{e}^{+}, \mathrm{e}^{-}\right)$pair is created and the orbital electron is also ejected from the atom. The three particles share the available kinetic energy and any distribution of that energy is possible as long as the energy is conserved. The process is then referred to as triplet production and has a photon energy threshold of $4 m_{\mathrm{o}} c^{2}=2.044 \mathrm{MeV}$.

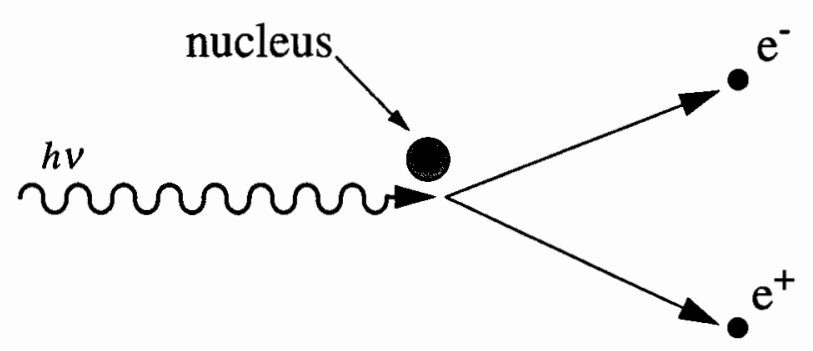

Figure 2-3. Schematic diagram of the pair production process. The incoming photon of energy $h v$ is totally absorbed in the Coulomb field of an atomic nucleus and an electron-positron pair $\left(\mathrm{e}^{+}, \mathrm{e}^{\mathrm{e}}\right)$ is created.

\section{II.D. Photonuclear reactions}

Photons can also be absorbed by the atomic nucleus while a nucleon is emitted, resulting in photo-disintegration or a photonuclear reaction. Like pair production, this effect can only occur at photon energies above a certain threshold value. The kinetic energy of the ejected nucleon must equal the incoming photon energy minus the nucleon's binding energy. In the clinical photon energy range the probability of such reactions is many orders of magnitude smaller than the combined probability of Compton, photoelectric and pair production processes and therefore is not of much interest in medical physics. 


\section{POST-INTERACTION PROCESSES}

The ejection of an orbital electron from a shell by supplying the electron an energy exceeding the shell's ionization potential, causes ionization of the atom, transforming the atom into a positive ion. Compton effect, photoeffect and triplet production produce positive ions by ejecting an orbital electron from an atom. Two other effects in nuclear physics, the K-capture and internal conversion also produce vacancies in atomic shells as do Coulomb interactions between charged particles and orbital electrons.

The atom eventually reverts to the ground state by filling the shell vacancy by one of the electrons from atomic shells with lower binding energies, and the energy difference either is emitted from the ion in the form of a photon (characteristic radiation) or is transferred to an electron in higher level shells causing its emission from the atom (Auger effect). The initial electron vacancy and the vacancies caused through the Auger effect eventually cascade to the outermost shell of the atom and are filled with neighboring free electrons to return the ion to the original neutral atomic state. Shell vacancies in high $Z$ materials are preferably followed by characteristic $\mathrm{x}$-ray photons, whereas in lower $Z$ materials they are preferably followed by emission of Auger electrons.

\section{III.A. Characteristic radiation}

$\mathrm{X}$-ray photons emitted through the transition of an electron from an outer shell to a vacancy in an inner shell are called characteristic radiation (also fluorescent radiation). The energy $h v$ of the characteristic photon is simply the difference in binding energies of the initial and final electron shells, i.e., $h v=E_{f}-E_{i}$. Therefore, if the vacancy is initially in the $\mathrm{K}$-shell and an electron from the L-shell moves in to fill that vacancy, the characteristic photon energy is given as $h v=E_{K}-E_{L}$. The photon is called a $K_{\alpha}$ photon as shown schematically in Fig. 2-4. Following this characteristic emission, not all energy of the excited atom is released because there is now a vacancy in the L-shell. This vacancy can be filled by an electron from a higher shell producing another characteristic radiation $\left(L_{\alpha}\right.$ in Fig. 2-4) and so on, until a thermal electron is captured from a neighboring ion and the 
vacancy disappears. The other possibility for an atom to release energy from an excited state is to eject an Auger electron.

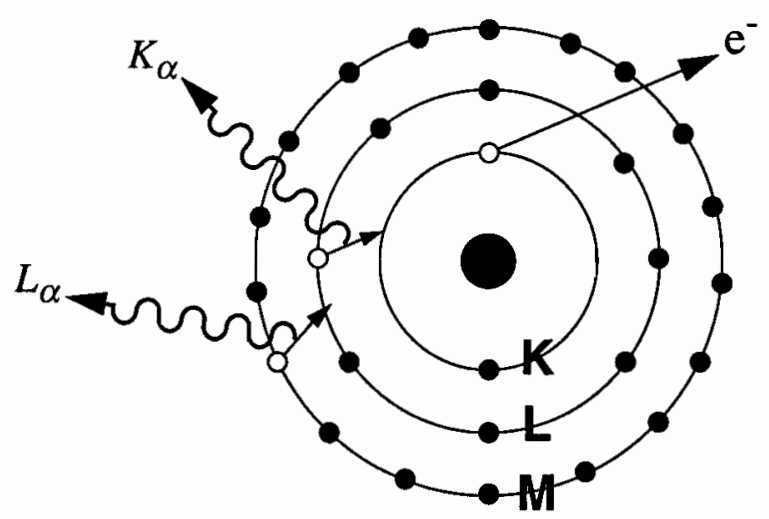

Figure 2-4. Schematic diagram of the emission of a characteristic photon $K_{\alpha}$ of energy $h v_{1}=E_{K}-E_{L}$ followed by the emission of a characteristic photon $L_{\alpha}$ of energy $h v_{2}=E_{L}-E_{M}$.

\section{III.B. Auger electrons}

The emission of Auger electrons is a competing process to the emission of characteristic photons. An excited atom ejects one or more orbital electrons, called Auger electrons, to release some of the excess energy. The Auger electron can originate from the same shell (referred to as a Coster-Kronig electron) or from a higher shell than the shell of the electron that filled the initial vacancy, and will have a total kinetic energy $T_{e^{-}}=E_{1}-E_{2}-E_{3}$, where $E_{1}$ is the binding energy of the initial vacancy shell, $E_{2}$ is the binding energy of the donor electron, and $E_{3}$ is the binding energy of the shell from which the Auger electron is ejected. Figure 2-5 shows an example where the initial vacancy is in the K-shell, an electron from the L-shell is filling the electron hole, and an Auger electron from the M-shell is emitted with a kinetic energy $T_{e^{-}}=\left|E_{K}\right|-\left|E_{L}\right|-\left|E_{M}\right|$. 


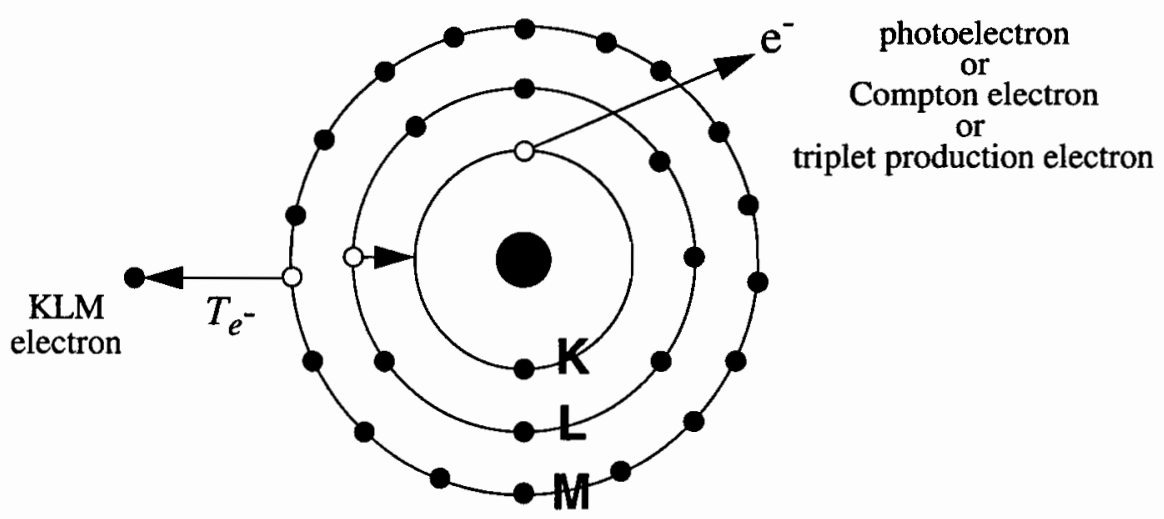

Figure 2-5. Schematic diagram of a KLM Auger electron emission. An initial vacancy in the K-shell is created, an electron from the L-shell fills the vacancy, and an Auger electron is emitted from the M-shell (KLM Auger electron). The Auger electron is given a total kinetic energy $T_{e^{-}}=\left|E_{K}\right|-\left|E_{L}\right|-\left|E_{M}\right|$.

\section{III.C. Fluorescence yield}

The probability for emission of a characteristic photon, following the transition of an orbital electron from an outer atomic shell to an inner atomic shell, is known as the fluorescence yield $\omega$. Each electron shell has its own fluorescence yield; $\omega_{K}$ and $\omega_{L}$ are fluorescence yields for the $\mathrm{K}$ - and $\mathrm{L}$ shells, respectively, and are shown in Fig. 2-6 plotted against the atomic number $Z$ of the medium. For a given shell, the fluorescence yield value represents the number of characteristic photons emitted per vacancy in that shell. A fluorescent yield of $\omega=1$ implies a certainty for emission of characteristic radiation, while a fluorescent yield of $\omega=0$ implies a certainty for Auger effect to result from a shell vacancy. $\omega_{K}$ is zero for $Z<10$, increases rapidly with increasing $Z$, reaches 0.5 at $Z=30$ and approaches unity for large $Z$. $\omega_{L}$ is nearly zero for $Z<30$ and increases to 0.42 for large $Z$ (Ref. \#3). For high $Z$ materials with vacancies in the $\mathrm{K}$ - and $\mathrm{L}$-shells, characteristic $\mathrm{x}$ rays will play an important role in releasing the excess energy. The probability of a characteristic $\mathrm{x}$ ray emission is negligible when filling a vacancy in a higher shell than the $\mathrm{K}$ - or $\mathrm{L}$ shells; emission of an Auger electron is by far more likely to be the mechanism by which the atom releases its excess energy. 


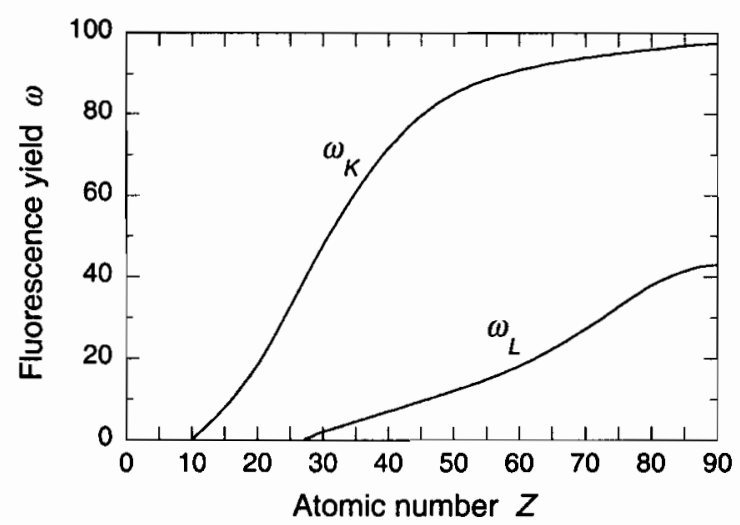

Figure 2-6. Fluorescence yield $\omega$ for the $\mathrm{K}$ and $\mathrm{L}$ shells. $\omega_{k}$ is calculated from Lederer and Shirley ${ }^{4}$ and $\omega_{L}$ from Burhop 5 .

\section{III.D. Positron annihilation}

The positrons produced by pair or triplet production move through the absorber and lose their kinetic energy as described in Chapter 3. However, when the positron kinetic energy is spent, the positron undergoes annihilation with a neighboring electron, and two photons are produced. To conserve energy and momentum the two photons have an energy of $0.511 \mathrm{MeV}$ each and move in opposite directions. Most commonly the positron's fate follows the two $0.511 \mathrm{MeV}$ annihilation process. However, there is also a small probability for annihilation in flight in which photons with energies larger than $0.511 \mathrm{MeV}$ are produced.

\section{ATtEnUATION COEFFICIENTS}

The various interaction processes through which a photon may be absorbed or scattered were described in the previous sections of this chapter. Each of these interactions has a given probability or cross section which varies with photon energy $h v$ and atomic number $Z$ of the absorber material. 


\section{IV.A. Cross sections and attenuation coefficients}

The likelihood that an interaction takes place for a given type of particle at a given energy impinging into a particular medium of atomic number $Z$ is expressed by the concept of reaction cross section $\sigma$. The reaction cross section for a particular interaction corresponds to an effective area that is presented to the incident radiation (Fig. 2-7) and has SI units of $\mathrm{m}^{2}$. However, a more convenient unit is the barn " $\mathrm{b}$ " equal to $10^{-28} \mathrm{~m}^{2}$. This area is a measure of an interaction probability rather than a geometrical cross sectional area $\sigma_{g}$ of a nucleus $\pi R^{2}$ of radius $R$. This probability area corresponds to an imaginary disk perpendicular to the incident beam; if a particle impinges upon any part of the disk, a reaction will occur, and no interaction takes place if the particle's path falls outside the target zone. The total interaction cross section is a sum of all cross sections for the various possible interaction processes.

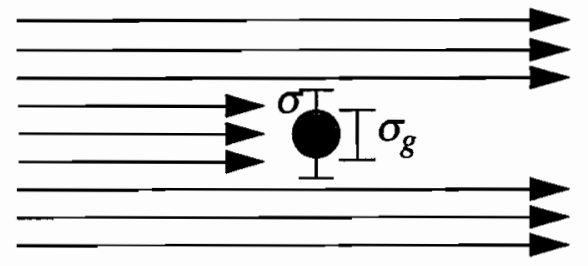

Figure 2-7. Schematic representation of the cross section, illustrating the difference between the cross section $\sigma$ and the geometrical cross section $\sigma_{g}$.

\section{IV.A.1 Electronic and atomic cross section}

For processes that have an electron as the centre of interaction, Compton interaction for example, it is possible to express the fundamental interaction as a cross section per electron or electronic cross section $e^{\sigma}$, expressed in $\mathrm{cm}^{2} /$ electron. For a material of atomic number $Z$ it is possible to express the cross section per atom, or atomic cross section ${ }_{a} \sigma$ which has dimensions of $\mathrm{cm}^{2} /$ atom. For a given material of atomic number $Z$, containing $Z$ electrons per atom, the atomic cross section is equal to the electronic cross section multiplied by $Z$; i.e., ${ }_{a} \sigma={ }_{e} \sigma \cdot Z$. 


\section{IV.A.2 Linear attenuation coefficient}

On many occasions, in particular for shielding calculations and diagnostic imaging, it is useful to know by what fraction the incident beam photon fluence will be attenuated by a given thickness of material, hence the utility of a linear attenuation coefficient $\mu$. This coefficient can be derived from the atomic or electronic cross sections, $\mu=N_{a} \sigma=Z N_{e} \sigma$, where $N_{a}$ is the number of atoms per $\mathrm{cm}^{3}$ (concentration of atoms) given by $N_{a}=\rho\left(N_{A} / A\right)$, with $N_{A}$ the Avogadro's number $\left(6.022 \times 10^{23}\right.$ atom/gram-atom), $A$ the atomic mass in g/gram-atom, and $\rho$ the material density in $\mathrm{g} / \mathrm{cm}^{3}$.

As monoenergetic and monodirectional photons of initial photon fluence $\phi(0)$ (number of photons per $\mathrm{cm}^{2}$ ) penetrate the absorber, some photons will be transmitted, some scattered, and some absorbed. Let $\phi(x)$ be the fluence at depth $x$ with no interaction. The number of photons that interact within a small gap $\mathrm{d} x$ is proportional to $\phi(x)$ and to $\mathrm{d} x$, with the constant of proportionality $\mu$ the linear attenuation coefficient:

$$
\mathrm{d} \phi=-\mu \phi(\mathrm{x}) \mathrm{d} x
$$

The solution of Eq. (2-7) is:

$$
\phi(x)=\phi(0) e^{-\mu x}
$$

indicating that the beam is attenuated exponentially with thickness of attenuator $x$.

For monoenergetic photon beams the half value layer $(H V L)$ may be defined as the attenuator thickness that attenuates the photon beam intensity to $50 \%$ of its original value; i.e., $H V L=\ln 2 / \mu$. Similarly, the tenth value layer $(T V L)$ is defined as the thickness of the attenuator that attenuates the photon beam intensity to $10 \%$ of its original value; i.e., $H V L=\ln 10 / \mu$. Attenuation of photon beams which possess a spectral distribution, on the other hand, is more complicated because of the dependence of $\mu$ on the photon energy. This dependence causes changes in the photon spectrum as more and more layers of the attenuator are traversed. These spectral changes may manifest themselves as beam hardening in the orthovoltage and superficial energy range and as beam softening in the megavoltage energy range. 


\section{IV.A.3 Mass attenuation coefficient}

A thickness $x$ of an absorber of density $\rho$ will attenuate the fluence of the beam by the same amount as thickness $x / 2$ of an absorber, made of the same material of density $2 \rho$, since the linear attenuation coefficient is dependent on the density of the absorbing medium. The linear attenuation coefficient in Eq. (2-8) can be replaced with a mass attenuation coefficient $(\mu \rho)$ for which there is no density dependence:

$$
\phi(X)=\phi_{o} e^{-\left(\frac{\mu}{\rho}\right) X}
$$

where $X=\rho x$, the mass thickness of the absorber in $\mathrm{g} / \mathrm{cm}^{2}$. The mass attenuation coefficient is expressed in $\mathrm{cm}^{2} / \mathrm{g}$, and represents the probability of an interaction per $\mathrm{g} / \mathrm{cm}^{2}$ of the material traversed.

\section{IV.B. Photoelectric cross section}

The exact theoretical evaluation of the photoelectric cross section presents great difficulty. Franz's theory ${ }^{6}$ derives the atomic differential photoelectric cross section formula, and various solutions to the problem have been discussed in the literature ${ }^{7,8}$ with a summary given by Pratt ${ }^{9}$. Empirical values of the photoelectric cross section have been published and are completed by theoretical interpolations for various photon energies and absorbing media.

A photoelectric interaction results in a complete absorption of the incident photon by a bound electron, in particular by one of the innermost shells since momentum conservation precludes absorption by unbound electrons. As a result of that rule, about 80 percent of the photoelectric absorbing events occur with electrons in the K-shell, as long as the energy of the photon exceeds the energy of bound electrons.

The atomic photoelectric cross section ${ }_{a} \tau$ as a function of the incident photon energy $h v$ displays a sawtooth structure, as shown in Fig. 2-8 for a lead absorber. The sharp discontinuities, known as absorption edges, arise whenever the incident photon energy coincides with the ionization energy of electrons in the $\mathrm{K}, \mathrm{L}, \mathrm{M}, \ldots$ shells. For a photon 
with energy just below a particular shell binding energy no interaction is possible with electrons of that shell. As the energy of the photon is increased to an equal or greater value than the shell binding energy, an interaction becomes possible increasing suddenly the probability of interaction and producing the sawtooth structure in the cross section curve. The sawtooth shape also evinces the fine structure of the shells, since each shell, with the exception of the K-shell, possesses subshells having slight variations in binding energy.

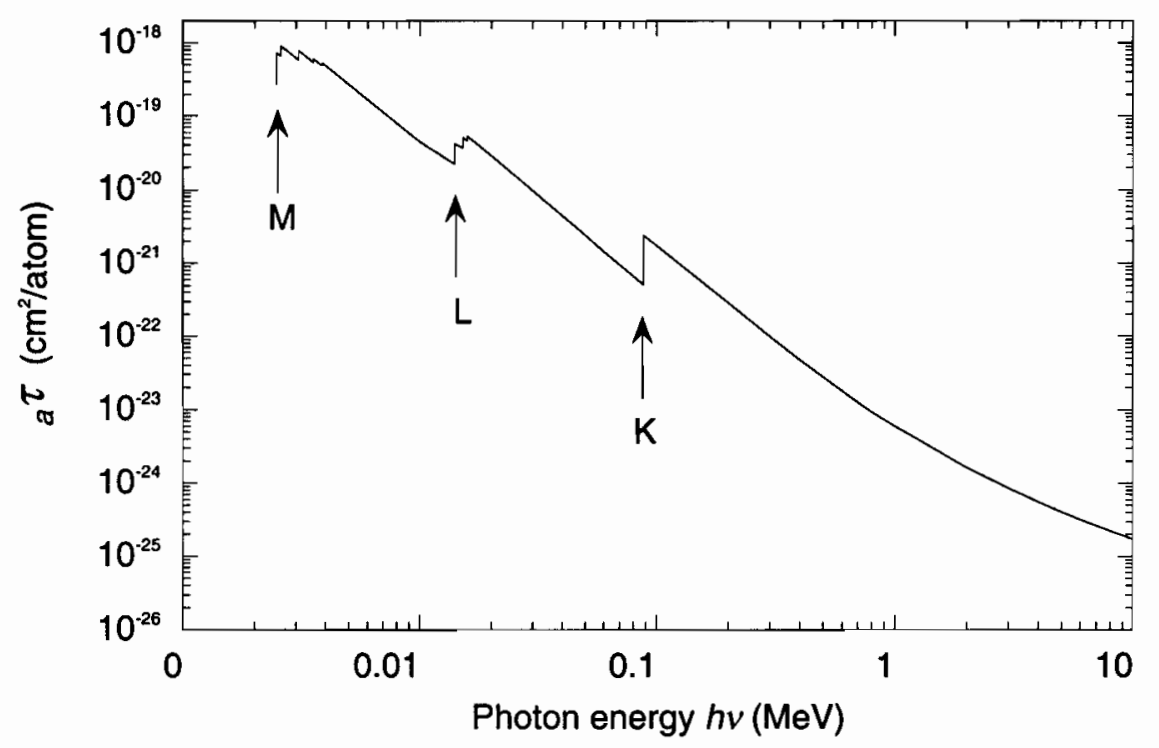

Figure 2-8. The photoelectric atomic cross section for lead illustrating the discontinuities known as the K, L, and $\mathrm{M}$ absorption edges as well as the subshell discontinuities.

In the energy region $h v \leq 0.1 \mathrm{MeV}$ photoeffect is important for photons with energies greater than the atomic binding energy of the atomic electrons of the absorber, and the atomic cross section ${ }_{a} \tau$ varies roughly as $Z^{4}$ and $(h v)^{-3}$, i.e.,

$$
\tau \tilde{\propto} \frac{Z^{4}}{h v^{3}}
$$

For the photoelectric mass attenuation coefficient $\tau / \rho$ this becomes:

$$
\frac{\tau}{\rho} \tilde{\propto}\left(\frac{Z}{h v}\right)^{3}
$$

At photon energies above $5 \mathrm{MeV}$ the atomic photoelectric cross section ${ }^{3}$ varies as $(h v)^{-1}$. 


\section{IV.C. Thompson scattering (classical scattering)}

The earliest classical electromagnetic theoretical description of photon-electron scattering was given by J.J. Thompson ${ }^{7}$. The subsequent Compton scattering is well approximated by Thompson scattering theory at low photon energies ${ }^{3}$ where $h v<0.01$ $\mathrm{MeV}$. Thompson scattering theory assumes the electron to be free to oscillate under the influence of the electric vector of the incoming photon, while absorbing the photon's energy and hastily emitting another photon of the same energy possibly in a different direction. No energy is retained by the electron as a result of this elastic event. The differential cross section per unit solid angle per electron for a photon scattered at an angle $\theta$ was shown by Thompson to be:

$$
\frac{d_{\mathrm{e}} \sigma}{d \Omega}=\frac{r_{0}^{2}}{2}\left(1+\cos ^{2} \theta\right)
$$

where $r_{0}=e^{2} /\left(4 \pi \varepsilon_{0} m_{0} c^{2}\right)=2.818 \times 10^{-13} \mathrm{~cm}$ is the classical electron radius. The total cross section per electron:

$$
e^{\sigma_{0}}=\frac{8 \pi r_{0}^{2}}{3}=6.65 \times 10^{-25} \mathrm{~cm}^{2} / \text { electron }
$$

is obtained by integrating Eq. (2-12) over the solid angle $d \Omega$.

\section{IV.D. Rayleigh scattering (coherent scattering)}

Rayleigh scattering ${ }^{10}$ is produced by the interaction of a photon with an atom. Photons are scattered by the bound electrons of the atom while none of the photon energy is transferred to the atom. This process principally occurs at low photon energies in large $Z$ materials. The differential cross section in angle $\theta$ for coherent scattering is obtained by expanding the solid angle $\mathrm{d} \Omega$ of Eq. (2-12) in terms of $\theta$ and multiplying the result by the atomic form factor ${ }^{11,12} F(x, z)$ : 


$$
\frac{d \sigma_{c o h}}{d \theta}=\frac{r_{0}^{2}}{2}\left(1+\cos ^{2} \theta\right) F(x, z) 2 \pi \sin \theta
$$

where the parameter $\mathrm{x}=\sin (\theta / 2) / \lambda$.

\section{IV.E. Compton cross section (incoherent scattering)}

Klein and Nishina ${ }^{13}$ expanded Thompson's theory to deal with Compton scattering by applying Dirac's relativistic theory of the electron. The improvement corrected the previous constant value of ${ }_{e} \sigma_{0}=6.65 \times 10^{-25} \mathrm{~cm}^{2} /$ electron that was known to be too large for $h v>0.01 \mathrm{MeV}$. In Klein-Nishina (K-N) theory, the electron is still assumed to be at rest and unbound, while $e_{e}^{\sigma}$ varies with $h v$ and follows well the experimental cross section values. The Klein-Nishina expression for the differential cross section per unit solid angle per electron for a photon scattered at an angle $\theta$ is:

$$
\frac{d_{e} \sigma}{d \Omega}=\frac{r_{0}^{2}}{2}\left(\frac{h v^{\prime}}{h v}\right)^{2}\left(\frac{h v}{h v^{\prime}}+\frac{h v^{\prime}}{h v}-\sin ^{2} \theta\right)
$$

where $h v^{\prime}$ is given by Eq. (2-3). For low photon energies, $h v \approx h v^{\prime}$ and Eq. (2-15) reduces to Thompson's equation (Eq. (2-12)), as expected. The total cross section per electron $e^{\sigma}$ is obtained by integrating Eq. (2-15) over all scattering angles $\theta$ :

$$
e^{\sigma}=2 \pi r_{0}^{2}\left\{\frac{1+\varepsilon}{\varepsilon^{2}}\left[\frac{2(1+\varepsilon)}{1+2 \varepsilon}-\frac{\ln (1+2 \varepsilon)}{2 \varepsilon}\right]+\frac{\ln (1+2 \varepsilon)}{2 \varepsilon}-\frac{1+3 \varepsilon}{(1+2 \varepsilon)^{2}}\right\}\left(\frac{\mathrm{cm}^{2}}{\text { electron }}\right)
$$

where $\varepsilon=h v / m_{0} c^{2}$

Figure 2-9 shows the Klein-Nishina electronic cross section $e^{\sigma}$ as a function of photon energy $h v$. As expected, $e_{e} \sigma$ is almost equal to the Thompson scattering cross section at $h v \leq 0.01 \mathrm{MeV}$ but then it gradually decreases at higher photon energies and becomes inversely proportional to the photon energy at large $h v$, i.e., $e^{\sigma \propto(h v)^{-1}}$. Since Klein-Nishina's theory assumes the electron to be unbound, the electronic cross section is independent of the atomic number of the medium $Z$. 
The atomic $\mathrm{K}-\mathrm{N}$ cross section is proportional to $Z$, since ${ }_{a} \sigma=Z \cdot{ }_{e} \sigma$ ( $\mathrm{cm}^{2} /$ atom $)$. The mass attenuation coefficient $\sigma / \rho$ is approximately independent of $Z$, since $\sigma / \rho=\left(N_{A} \cdot(Z / A)\right)_{e} \sigma\left(\mathrm{cm}^{2} / \mathrm{g}\right)$, where the ratio $Z / A$ is equal to 1 for hydrogen and may be averaged to $0.45 \pm 0.05$ for all other elements.

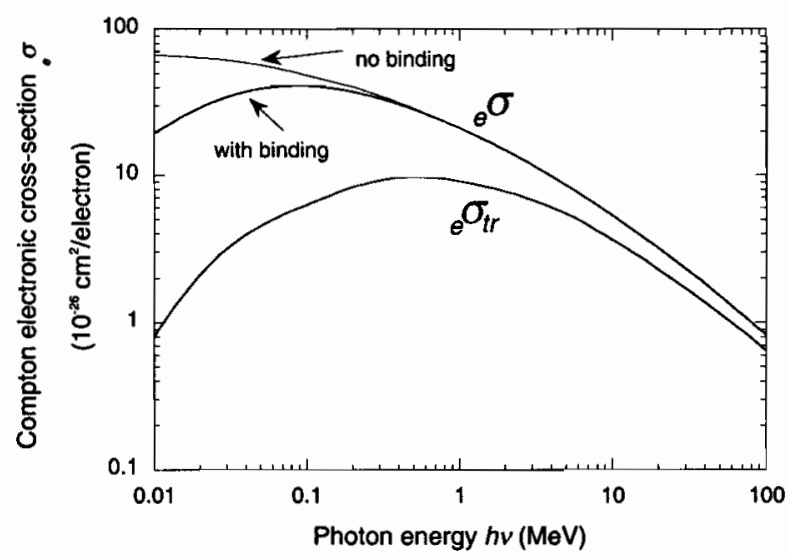

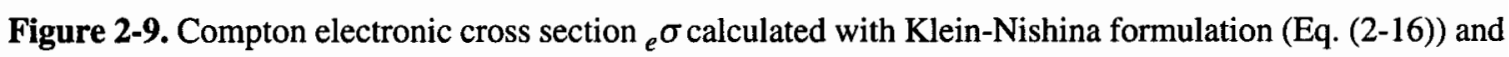
energy transfer coefficient $e_{e} \sigma_{t r}$ as a function of incoming photon energy $h v$.

\section{IV.F. Pair production cross section}

The Bethe and Heitler theory ${ }^{3,14}$ describes the pair production atomic differential cross section $d_{\mathrm{a}} \kappa$ as:

$$
\frac{d_{a} \kappa}{d T_{e^{+}}}=\frac{r_{0}^{2}}{137} \frac{Z^{2} \bar{P}}{h v-2 m_{0} c^{2}}
$$

where $T_{e^{+}}$is the kinetic energy of the positron and $\bar{P}$ is a function of $h v$ and $Z$. The dependence on $Z$ is small and generally ignored. 
The total interaction coefficient is obtained by integrating Eq. (2-17) over all possible $T_{e^{+:}}$

$$
\begin{aligned}
{ }_{a} \kappa & =\frac{r_{0}^{2}}{137} Z^{2} \int_{0}^{\left(h v-2 m_{0} c^{2}\right)} \frac{\bar{P}}{h v-2 m_{0} c^{2}} d T_{e^{+}} \\
& =\frac{r_{0}^{2}}{137} Z^{2} \int_{0}^{1} \bar{P} d\left(\frac{T_{e^{+}}}{h v-2 m_{0} c^{2}}\right) \\
& =\frac{r_{0}^{2}}{137} Z^{2} \bar{P} .
\end{aligned}
$$

Therefore, ${ }_{a} \kappa$ is proportional to the atomic number squared, since $\bar{P}$ is independent of $Z$. $\bar{P}$ varies approximately as a logarithm function of $h v$ tending to become a constant value, independent of $h v$, for very large $h v$ because of the electron screening of the nuclear field, as shown in Fig. 2-10 for a lead absorber. The pair production mass attenuation coefficient $\kappa / \rho$ is then proportional to $Z$, since $\kappa / \rho={ }_{a} \kappa\left(N_{A} / A\right)$ and $Z / A$ is a constant, as discussed above.

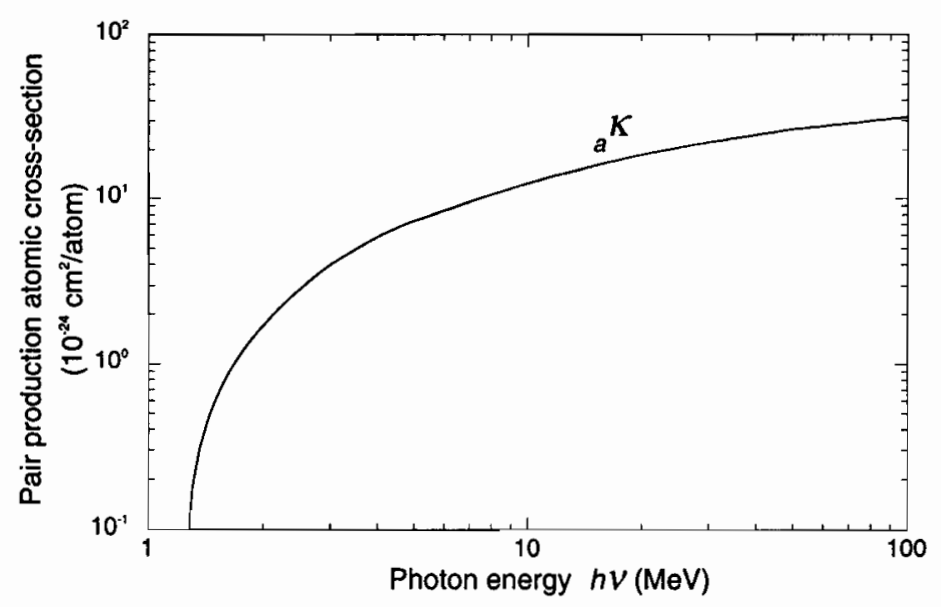

Figure 2-10. Pair production atomic cross section ${ }_{a} K$ as a function of the incident photon energy $h v$ in lead. 


\section{IV.G. Total attenuation coefficient}

The total mass attenuation coefficient $\mu / \rho$ is the sum of the individual coefficients, principally the photoelectric $\tau / \rho$, Compton $\sigma / \rho$ and pair-production $\kappa / \rho$ :

$$
\frac{\mu}{\rho}=\frac{\tau}{\rho}+\frac{\sigma_{R}}{\rho}+\frac{\sigma}{\rho}+\frac{\kappa}{\rho}
$$

Coherent scattering is only important at very low photon energies. Photonuclear reactions are neglected, because their cross sections are many orders of magnitude lower than those for the three processes described above. Figure 2-11 shows the contribution of the main interactions (photoeffect, Compton and pair production) to the total mass attenuation coefficient (solid line) in a water absorber. It is clear that photoeffect is mainly important at low photon energies, while Compton effect is relatively important across the whole range of photon energies, and pair production is important at high photon energies.

Figure 2-12 shows the total atomic cross section coefficient as a function of photon energy for three different materials (lead, copper and water). It shows the presence of the absorption edges for high $Z$ materials (lead and copper) at low energies where photoeffect is important, whereas for low $Z$ material (water) no edges are seen because they occur at photon energies below $10 \mathrm{keV}$. At high energies we clearly see that the contribution from the pair production process is greater for high $Z$ materials.

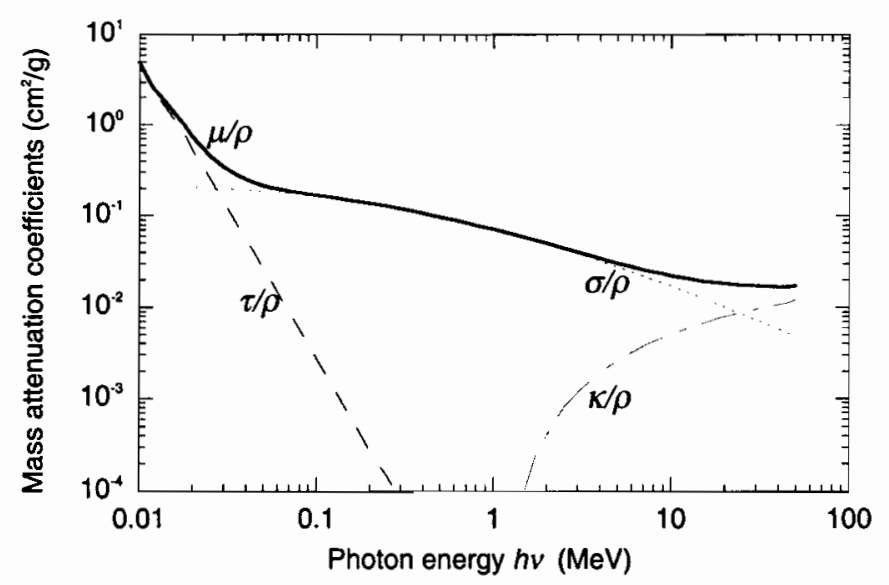

Figure 2-11. Total mass attenuation coefficient $\mu / \rho$ and the partial contribution of the photoeffect $\tau / \rho$, Compton $\sigma / \rho$, and pair-production $\kappa / \rho$ cross-sections for water as a function of photon energy. 


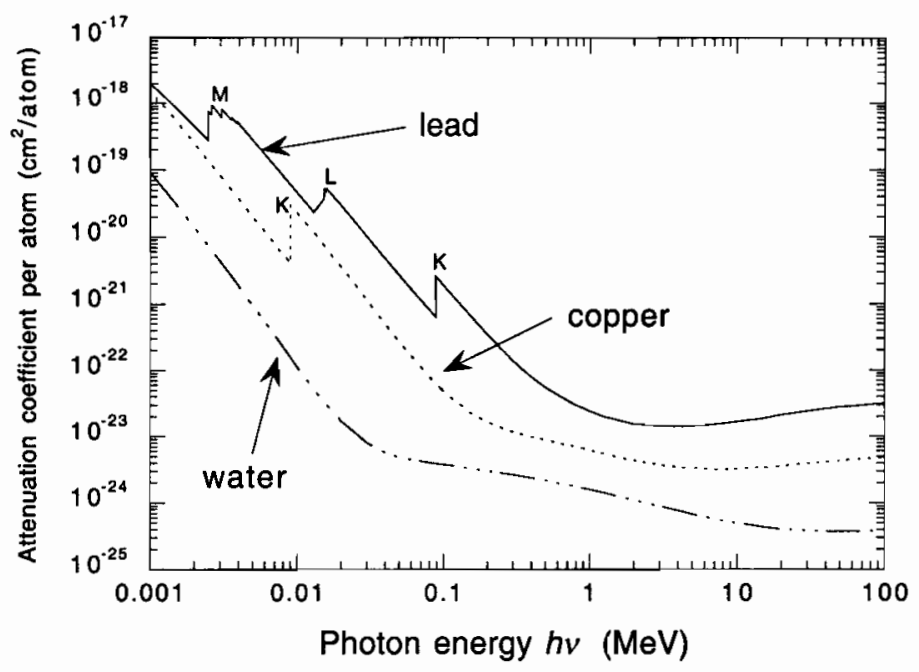

Figure 2-12. Total atomic attenuation coefficients $\mu_{a}$ for three different media: water, copper and lead.

\section{IV.H. Summary of interactions}

The atomic number $Z$ of the attenuator and the photon energy $h v$ are the parameters that dictate the importance of the attenuation by influencing the values of the cross sections of the various interactions taking place in the medium. Large $Z$ media have dense electron clouds surrounding their nuclei and the dominating interaction at low photon energies, close to the binding energy $E_{b}$ of the electrons ( $h \vee \tilde{>} E_{b}$ ), is the photoelectric effect because it involves an interaction of the photon with an entire atom. At higher photon energies, above the $1.022 \mathrm{MeV}$ threshold, and in the presence of a sufficiently strong Coulomb field (for relatively high $Z$ ) the photon will penetrate the electron cloud and pair production becomes the most important interaction. For media with lower atomic number $Z$ and $h \vee \gg E_{b}$, the electrons are considered almost unbound or "free" and the Compton effect is the dominating interaction process.

Table 2-1 illustrates the dependence of the atomic and electronic cross sections and the linear and mass attenuation coefficients on photon energy $h v$ and absorber atomic number $Z$ for the three major processes of photon interaction in the radiotherapy energy range (1 to $25 \mathrm{MeV}$ ). 
Table 2-1. Dependence of the photoelectric, Compton and pair production processes on $h v$ and $Z$. The electronic and atomic cross section and the linear and mass attenuation coefficient dependence is shown.

\begin{tabular}{|c|c|c|c|}
\hline & Photoelectric & Compton & Pair Production \\
\hline \hline electronic cross section & $e^{\tau \tilde{\propto} \frac{Z^{3}}{(h v)^{3}}}$ & $e^{\sigma \propto \frac{Z^{0}}{h v}}$ & $e^{\kappa \tilde{\propto} Z \ln (h v)}$ \\
\hline atomic cross section & ${ }_{\mathrm{a}} \tau \tilde{\propto} \frac{Z^{4}}{(h v)^{3}}$ & ${ }_{a} \sigma \propto \frac{Z^{1}}{h v}$ & $a^{\kappa \tilde{\propto} Z^{2} \ln (h v)}$ \\
\hline linear attenuation coefficient & $\tau \tilde{\propto} \frac{\rho Z^{3}}{(h v)^{3}}$ & $\sigma \tilde{\propto} \frac{\rho Z^{0}}{h v}$ & $\kappa \tilde{\propto} \rho Z \ln (h v)$ \\
\hline mass attenuation coefficient & $\frac{\tau}{\rho} \tilde{\propto} \frac{Z^{3}}{(h v)^{3}}$ & $\frac{\sigma}{\rho} \tilde{\propto} \frac{Z^{0}}{h v}$ & $\frac{\kappa}{\rho} \tilde{\propto} Z \ln (h v)$ \\
\hline
\end{tabular}

The relative importance of Compton scattering, pair production and photoeffect exhibit a strong dependence on photon energy $h v$ and of atomic number $Z$ of the absorbing medium. Figure 2-13 illustrates the relative importance of the three interaction mechanisms with a plot of $Z$ as a function of $h v$. The three regions delineated by the two solid curves represent the zones in which each mechanism predominates. The two mechanisms separated by a solid curve are of equal importance along that curve, since atomic interaction coefficients of neighboring effects are equal. The graph shows that Compton interactions predominate for low $Z$ materials, such as water and human tissues, for a broad range of photon energies. However, as $Z$ is increased at lower photon energies, the photoelectric effect becomes the most important mechanism as a result of the energy of the photon becoming close to the binding energies of electrons in the attenuator. At higher photon energies, pair production becomes the dominant effect as $Z$ is increased. 


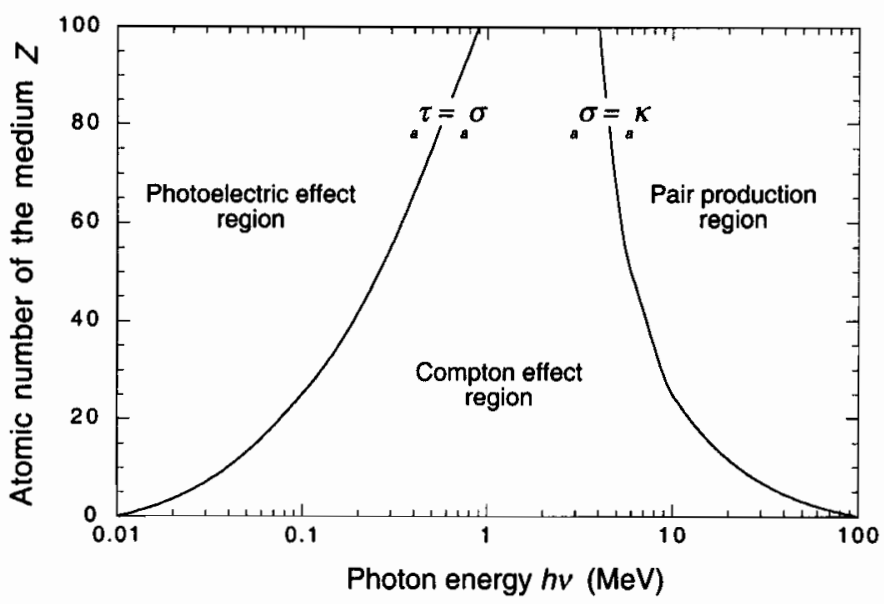

Figure 2-13. Relative importance of the three main photon interactions with matter as a function of the photon energy $h v$ and the atomic number $Z$ of the medium. The solid curves represent the values of $h v$ and $Z$ for which two interactions are of equal importance, i.e., atomic interaction coefficients of effects are equal.

\section{IV.I. Mass energy-transfer coefficient}

In dosimetry we are interested in the energy absorbed by matter exposed to radiation. This quantity is related to the mass attenuation coefficient. The mass attenuation coefficient describes the fraction of the incident photons that interact with the medium and are removed from the initial photon fluence. Similarly, the fraction of the incident photon energy that is transferred from the incident photons to secondary electrons in the medium is found by a mass energy-transfer coefficient $\mu_{t r} / \rho$. The mass energy-transfer coefficient is related to the mass attenuation coefficient $\mu / \rho$ through the mean energy transferred to the electron $\bar{E}_{t r}$ :

$$
\frac{\mu_{t r}}{\rho}=\frac{\mu}{\rho} \cdot \frac{\bar{E}_{t r}}{h v} .
$$

The mean energy transferred is an average over a large number of interactions. The total mass energy-transfer coefficient $\mu_{t r} / \rho$ is the sum of the three individual mass energy-transfer coefficients (photoelectric $\tau_{t r} / \rho$, Compton $\sigma_{t r} / \rho$, and pair production $\kappa_{t r} / \rho$ ). 


\section{IV.I.1 Energy transfer in photoelectric effect}

The fraction of the photon energy $h v$ transferred to the photoelectron from a photoelectric interaction is given by:

$$
\frac{T_{\mathrm{e}}}{h v}=\frac{h v-E_{b}}{h v}
$$

where $E_{b}$ is the binding energy of the photoelectron. This quantity is an approximation of the total fraction of $h v$ transferred to electrons, since the fraction varies as $E_{b}$ is different for electrons of different shells, and most of the binding energy is emitted in the form of characteristic radiation. The presence of Auger electrons, on the other hand, will increase the amount of energy transferred to the medium and Eq. (2-21) will then underestimate the mean energy transferred to the medium. Therefore, the mean mass energy-transfer coefficient $\left(\bar{\tau}_{t r} / \rho\right)$ for photoeffect is given by:

$$
\left(\frac{\bar{\tau}_{t r}}{\rho}\right)=\frac{\tau}{\rho}\left(1-\frac{\delta}{h v}\right)
$$

where $\delta$ is the average energy emitted as fluorescence radiation following the photoelectric interaction given by:

$$
\delta=\sum_{i} P_{i} \omega_{i} h \bar{v}_{i}
$$

In Eq. (2-23) $P_{i}$ is the probability for a photoelectric interaction in the $i^{\text {th }}$ shell, $\omega_{i}$ is the fluorescence yield for that shell, and $h \bar{v}_{i}$ is the mean fluorescence x-ray energy from a photoelectric interaction in the $i^{t h}$ shell. The sum is run down to the shell, where $h v<E_{i}$ with $E_{i}$ the binding energy of that shell.

\section{IV.I.2 Energy transfer in Compton effect}

In a Compton interaction, the energy of the incident photon $h v$ is shared between the scattered photon energy $h v^{\prime}$ and the recoil electron kinetic energy $T$. To estimate the energy transfer coefficient one needs to know the overall mean energy fraction given to the 
electrons, averaged over all scattering angles $\bar{T} /(h v)$. Modifying Eq. (2-15) to obtain a differential Klein-Nishina energy-transfer cross section, $d_{e} \sigma_{t r} / d \Omega$ :

$$
\frac{d_{e} \sigma_{t r}}{d \Omega}=\frac{d_{e} \sigma}{d \Omega} \cdot \frac{T}{h v}=\frac{r_{0}^{2}}{2}\left(\frac{h v^{\prime}}{h v}\right)^{2}\left(\frac{h v}{h v^{\prime}}+\frac{h v^{\prime}}{h v}-\sin ^{2} \theta\right) \cdot \frac{h v-h v^{\prime}}{h v}
$$

where $\left(h v-h v^{\prime}\right) / h v$ is the fraction of the initial photon energy given to the recoil electron. Integrating Eq. (2-24) over all photon scattering angles $\theta$ from 0 to $180^{\circ}$ yields the Klein-Nishina energy transfer cross section:

$$
\begin{aligned}
e^{\sigma_{t r}}= & 2 \pi r_{0}^{2}\left[\frac{2(1+\varepsilon)^{2}}{\varepsilon^{2}(1+2 \varepsilon)}-\frac{1+3 \varepsilon}{(1+2 \varepsilon)^{2}}-\frac{(1+\varepsilon)\left(2 \varepsilon^{2}-2 \varepsilon-1\right)}{\varepsilon^{2}(1+2 \varepsilon)^{2}}\right. \\
& \left.+\left(-\frac{4 \varepsilon^{2}}{3(1+2 \varepsilon)^{3}}\right)-\left(\frac{1+2 \varepsilon}{\varepsilon^{3}}-\frac{1}{2 \varepsilon}\right) \ln (1+2 \varepsilon)\right]\left(\frac{\mathrm{cm}^{2}}{\text { electron }}\right)
\end{aligned}
$$

A plot of the Compton energy transfer cross section $e^{\sigma_{t r}}$ is given in Fig. 2-9. The mean energy given to the recoil electron $\bar{T}$ is then given by:

$$
\bar{T}=h v \cdot \frac{e^{\sigma_{t r}}}{e^{\sigma}}
$$

The maximum energy transfer to the recoil electron is found by setting $\theta=\pi$ in Eq. $(2-2)$. Both the maximum $T_{\text {max }} / h v$ and mean $\bar{T} / h v$ fractions of the incident photon energy are plotted in Fig. 2-14 as a function of the incident photon energy $h v$. The mean Compton mass energy transfer coefficient $\frac{\bar{\sigma}_{t r}}{\rho}$ is given by:

$$
\frac{\bar{\sigma}_{t r}}{\rho}=\frac{\bar{T}}{h v} \cdot \frac{\sigma}{\rho}
$$

For a given photon energy $h v, \bar{T}$ is found from Fig. 2-14. 


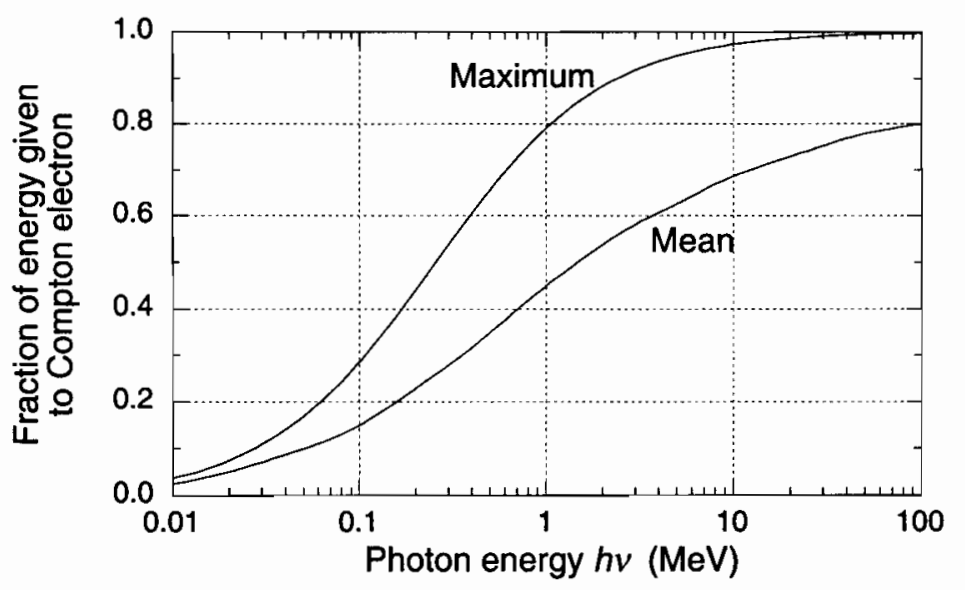

Figure 2-14. Maximum and mean fraction of the kinetic energy given to the recoil electron in a Compton interaction as a function of the incident photon energy $h v$.

\section{IV.I.3 Energy transfer in pair production}

The fraction of incoming photon energy transferred to kinetic energy given to charged particles $\left(\mathrm{e}^{-}, \mathrm{e}^{+}\right)$by a pair production event is calculated from Eq. (2-6) to be $\frac{h v-2 m_{o} c^{2}}{h v}$. Therefore, the mean mass energy transfer coefficient for pair production is given by:

$$
\frac{\bar{\kappa}_{t r}}{\rho}=\frac{\kappa}{\rho}\left(\frac{h v-2 m_{o} c^{2}}{h v}\right)
$$

\section{IV.I.4 Total mass energy transfer coefficient}

The total mass energy transfer coefficient, neglecting possible photonuclear reactions, is given by the sum of the mass energy transfer coefficients for the individual processes (photoeffect, Compton effect, and pair production):

$$
\frac{\bar{\mu}_{t r}}{\rho}=\frac{\bar{\tau}_{t r}}{\rho}+\frac{\bar{\sigma}_{t r}}{\rho}+\frac{\bar{\kappa}_{t r}}{\rho}
$$


Inserting Eq. (2-22), Eq. (2-27), Eq. (2-28) into Eq. (2-29), we obtain the total mean mass energy-transfer coefficient:

$$
\frac{\bar{\mu}_{t r}}{\rho}=\frac{1}{\rho h v}\left[\tau(h v-\delta)+\sigma \bar{T}+\kappa\left(h v-2 m_{o} c^{2}\right)\right]
$$

The mean energy transferred $\bar{E}_{t r}$ to charged particles through photon interactions is found by taking the ratio of Eq. (2-30) and Eq. (2-19) and multiplying by the photon energy $h v$ :

$$
\bar{E}_{t r}=\frac{\left(\frac{\bar{\mu}_{t r}}{\rho}\right)}{\left(\frac{\mu}{\rho}\right)} h v
$$

\section{IV.J. Total mass energy absorption coefficient}

The mass energy absorption coefficient $\left(\mu_{a b} / \rho\right)$ plays an important role in medical physics, since it leads to calculation of dose given to the medium, defined by the energy absorbed by unit mass in $\mathrm{J} / \mathrm{kg}$ (Ref. \#15). The mass energy-absorption coefficient is a direct function of the mass energy-transfer coefficient. The two coefficients are related through a quantity $g$ which corresponds to the average fraction of secondary-electron kinetic energy that is given up to radiative (bremsstrahlung) interactions with the medium:

$$
\frac{\mu_{a b}}{\rho}=\frac{\mu_{t r}}{\rho}(1-g)
$$

The radiative interactions within the medium are the bremsstrahlung for electrons and in-flight annihilation for positrons. Means for evaluating the bremsstrahlung fraction $g$ will be described in Chapter 3. As a rule of thumb, for low $h v$ and $Z, g \rightarrow 0$, since only a very small amount of secondary-electron kinetic energy is lost to radiative interactions and therefore $\mu_{a b} / \rho \approx \mu_{t r} / \rho$. As $h v$ and $Z$ increase, $g$ will also gradually increase; for example ${ }^{3}$ in lead at $h v=10 \mathrm{MeV}, g=0.37$. Similarly to the mean energy-transfer, the mean 
energy absorbed is found by taking a ratio of Eq. (2-32) and Eq. (2-19) and multiplying by the photon energy $h v$ :

$$
\bar{E}_{a b}=\frac{\left(\frac{\bar{\mu}_{a b}}{\rho}\right)}{\left(\frac{\mu}{\rho}\right)} h v
$$

\section{IV.K. Coefficients for compounds and mixtures}

For mediums that are mixtures of elements and compounds, the mass attenuation and energy-transfer coefficients are found from the Bragg rule ${ }^{3}$, such that:

$$
\left(\frac{\bar{\mu}}{\rho}\right)_{\text {total }}=\left(\frac{\bar{\mu}}{\rho}\right)_{a} W_{a}+\left(\frac{\bar{\mu}}{\rho}\right)_{b} W_{b}+\ldots
$$

where $W$ is the weight fraction of the elements $(a, b, \ldots)$ of the medium. This relation applies directly for the mass attenuations and energy-transfer coefficients. For the mass energy-absorption coefficient, it is a good approximation as long as the radiation yield ( $g$ value) is small, since electrons created by interaction with element $a$ will not only interact with element $a$ but also with element $b, c, \ldots$. Therefore, we can write an exact solution as

$$
\begin{aligned}
\left(\frac{\bar{\mu}_{a b}}{\rho}\right)_{\text {total }}= & \left(\frac{\bar{\mu}_{t r}}{\rho}\right)_{a}\left(1-W_{a} g_{a}-W_{b} g_{b}-\ldots\right) W_{a} \\
& +\left(\frac{\bar{\mu}_{t r}}{\rho}\right)_{b}\left(1-W_{a} g_{a}-W_{b} g_{b}-\ldots\right) W_{b}+\ldots
\end{aligned}
$$

Values of the various mass coefficients, as well as the $g$ values, are available from the litterature. ${ }^{16-19}$ 


\section{SUMMARY}

High energy photons, used for applications in medical physics, are primarily in the range of energies between 1 and $25 \mathrm{MeV}$. The three primary interactions between these photons and typical human tissues are photoelectric effect, Compton effect, and pair production. The relative importance of these interactions is a function of the photon energy $h v$ and the atomic number $Z$ of the absorbing tissues.

Following Compton, photoeffect and triplet production interaction, an orbital electron is ejected leaving the atom in an excited state with an electron shell vacancy. The excess energy will be released in the form of characteristic photons or by Auger electrons until the Coulomb field of the ionized atom captures a thermal electron to fill the vacancy.

Each interaction process has a probability which is directly related to the interaction cross section from which the various interaction coefficients are derived. There is an interaction coefficient for each possible process and the total interaction coefficient is a simple addition of each of these coefficients. The total coefficient can be expressed as linear, mass attenuation coefficient, mass energy-transfer coefficient and mass energy-absorption coefficient. The mass-energy absorption coefficient is the most important coefficient, since it is directly related to the absorbed dose in the absorbing material. 


\section{References}

1 George Neilson Whyte, Principles of radiation dosimetry (Wiley, New York, 1959).

2 J. R. Greening, Fundamentals of radiation dosimetry (A. Hilger in collaboration with The Hospital Physicists' Association, Bristol, England, 1981).

3 Frank H. Attix, Introduction to radiological physics and radiation dosimetry (Wiley \& Son, New York, 1986).

4 C. Michael Lederer, Virginia S. Shirley, and Edgardo Browne, Table of isotopes, 7th / ed. (Wiley, New York, 1978).

5 E. H. S. Burhop, The Auger effect and other radiationless transitions (University Press, Cambridge, 1952).

6 Pierre Marmier and Eric Sheldon, Physics of nuclei and particles (Academic Press, New York, 1969).

7 Robley Dunglison Evans, The atomic nucleus (R.E. Krieger, Malabar, Florida, 1982).

8 John Howard Hubbell, "Photon cross sections, attenuation coefficients, and energy absorption coefficients from $10 \mathrm{keV}$ to $100 \mathrm{GeV}$," Report NSRDS-NBS29, U.S. National Bureau of Standards, Washington D. C. (1969).

9 R. H. Pratt, “Atomic Photoelectric Effect at High Energies,” Phys. Rev. 117, 1017-1102 (1960). 
10 Harold Elford Johns and John Robert Cunningham, The physics of radiology, 4th ed. (Charles C. Thomas, Springfield, I1l., U.S.A., 1983).

11 E. F. Plechaty, D. E. Cullen, and R. J. Howerton, Tables and graphs of photon interaction cross sections form $1.0 \mathrm{keV}$ to $100 \mathrm{MeV}$ derived from LLL evaluated nuclear data library, UCRL-50400 (University of California, Lawrence Livermore Laboratory, Springfield, 1975).

12 John Howard Hubbell, W. J. Veigele, E. A. Briggs et al., "Atomic form factors, incoherent scattering functions and photon scattering cross section," J. Phys. Chem. Ref. Data 4, 471 (1975).

13 O. Klein and Y. Nishina, "Über die Streuung von Strahlung durch freie Elektronen nach der neuen relativistischen Quantendynamik von Dirac," Physik 52, 853-868 (1929).

14 H. Bethe and W. Heitler, "On the stopping of fast particles and on the creation of positive electrons," Proc. Roy. Soc. A. 146, 83-112 (1934).

15 ICRU, "Radiation quantities and units," ICRU Report 33, International Commission on Radiation Units and Measurements, Washington, D.C. (1980).

16 John Howard Hubbell, "Photon mass attenuation and energy-absorption coefficients from $1 \mathrm{keV}$ to $20 \mathrm{MeV}$," Int. J. Appl. Rad. Isot. 33, 1269 (1982).

17 John Howard Hubbell, H. A. Gimm, and I. Øverb $\varnothing$, "Pair, triplet and total cross sections for $1 \mathrm{MeV}-100 \mathrm{GeV}$ photons in elements $\mathrm{Z}=1-100$.," J. Phys. Chem. Ref. Data 9, 1023 (1980). 
18 William Herbert McMaster and Lawrence Radiation Laboratory., Compilation of x-ray cross sections (University of California Lawrence Radiation Laboratory, Livermore, California, 1969).

19 E. Storm and H. I. Israel, Photon cross sections from $1 \mathrm{keV}$ to $100 \mathrm{MeV}$ for elements from $Z=1$ to $Z=100$ (Academic Press, New York, 1970). 


\section{Chapter 3}

\section{Interactions of electrons with matter}

This chapter describes the various interactions by which electrons and positrons experience a change in energy and direction in passing through matter. In contrast to photons which can travel through a medium without interacting at all or interact in one or only a few events to lose all their energy, electrons or positrons lose their kinetic energy almost continuously as they slow down in matter. The Coulomb electronic field produces a force that makes the electrons interact with orbital electrons or nuclei along their path. Particles having kinetic energies of the order of $\mathrm{MeV}$ will experience some 100000 collisions before they are completely absorbed by the material. Therefore, their behavior in terms of trajectories and energy loss is described by statistical theories of multiple scattering (MS), including Rutherford cross section as well as Molière ${ }^{1,2}$, Mott $^{3}$ and Goudsmit-Saunderson MS distributions ${ }^{4,5}$.

In the first approximation, the electron and positron penetration into medium is described by the "continuous slowing down approximation" (CSDA) which assumes that the particles lose their energy linearly and continuously as they penetrate into the medium. The mean depth at which these particles come to rest and get absorbed is called the CSDA range. The range depends on the electron initial kinetic energy as well as on the composition of the absorber.

Electron and positron interactions with media, especially water and tissue, are very important in radiation dosimetry, since the energy they transfer to the medium is ultimately absorbed and determines the dose that is given to the medium. 


\section{TYPES OF INTERACTIONS}

Essentially, three interactions contribute to the slowing down of electrons as they pass through an absorber. They are:

Inelastic scattering on atomic orbital electrons constitutes the principal mode of interaction for the electrons. It leads to excitations and ionizations of atoms of the medium.

(ii) Elastic scattering on atoms in which the incident electron is scattered without any change in energy.

(iii) Inelastic nuclear scattering which causes energy loss through production of bremsstrahlung.

The electron Coulomb-force interactions can be described in terms of the relative size of the classical impact parameter $b$ with respect to the size of the classical atomic radius $a$, as illustrated in Fig. 3-1. The three possible cases are: "soft" collisions for which $b » a$, "hard" collisions for which $a-b$, and radiative collisions for which $b \ll a$.

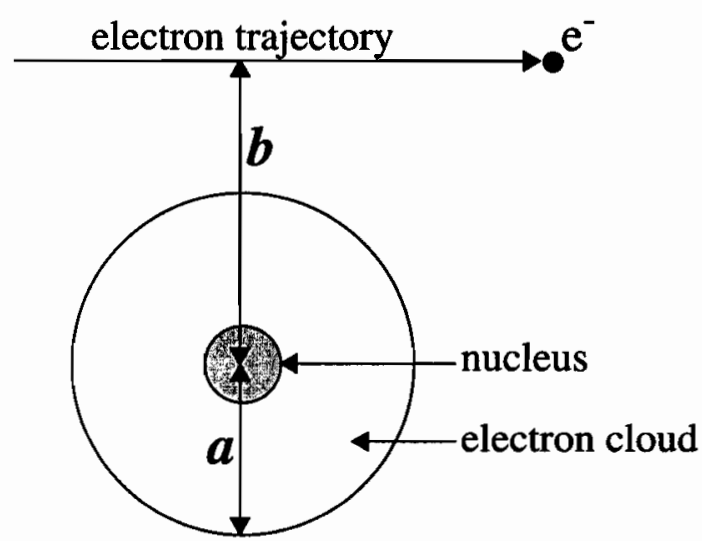

Figure 3-1. Parameters for an electron collision with atoms. ( $a$ ) is the classical radius of the atom and $(b)$ is the classical impact parameter.

\section{I.A. Soft collisions $(b>>a)$}

Soft collisions are produced by interactions of the electron Coulomb-force with the atom as a whole and this happens when the impact parameter $b$ is much larger than the 
atom radius $a$. The Coulomb force distorts the atom; in solids and liquids this distortion could produce a polarization or density effect which is important in radiation dosimetry. In general, the distortion results in either an excitation to a higher energy level of the atom or sometimes in an ejection of a valence shell electron. A soft collision is the most probable interaction of an energetic electron with an absorbing medium, since $b$ has a high probability to be greater than $a$. The electron loses a very small amount of energy during individual soft collisions (a few eV), however, it undergoes numerous soft collisions which combined account for about half of the total energy lost in the travelled path of an electron through an absorber.

\section{I.B. Hard collisions $(b \sim a)$}

When the impact parameter $b$ is on the order of the atomic radius $a$ the electron most likely interacts with a single atomic electron which receives most of the incident electron's kinetic energy. The result is the ejection of this atomic electron (called $\delta$ ray) with enough kinetic energy to travel further on a path of its own, while undergoing additional Coulomb collisions with the absorber atoms. In radiation dosimetry, the energy transferred to the $\delta$ ray is not considered to be absorbed locally.

In addition, if a hard collision causes an ejection of an inner-shell electron, the collision will be followed by emission of characteristic photons or by ejection of Auger electrons. Therefore some of the energy transferred to the medium by a hard collision will be carried away by photons or $\delta$ rays and will not contribute to the locally absorbed dose.

Given the size of $a$, the probability for a hard collision to occur is much smaller than that for a soft collision. However, the fraction of the incident electron energy transferred to the medium by a single hard collision is much higher than that for a single soft collision, and on average the total amount of energy transferred from an electron or a positron to the medium by hard collisions and by soft collisions is comparable. The probability for a hard collision depends on the quantum mechanical spin of the incident charged particles and therefore, the linear rate at which the particle loses its energy (stopping power) is different for a positron in comparison with an electron (see Section III). 


\section{I.C. Radiative collisions $(b<<a)$}

As the classical impact parameter $b$ gets much smaller than the classical atomic radius $a$, the incident electron is likely to interact with the nuclear Coulomb-field. In most of these interactions, $\sim 98 \%$ of these events, the electron will simply undergo an elastic collision, from which no secondary particles are created and no excitation of the atom takes place $^{6}$. This type of interaction only results in deflecting the electron from its original direction.

However, in the remaining $\sim 2 \%$ of the electron-nucleus interactions, the electron is scattered inelastically on an atomic nucleus, and experiences an important change of direction followed by an emission of a high energy photon. The electron is deflected and slowed down in the field of the nucleus and the emitted photons can carry away up to $100 \%$ of the kinetic energy of the electron. The emitted photons are called bremsstrahlung (which means "braking radiation" in German) and they are likely to carry their energy outside the absorbing medium, when their energy is high enough to escape the medium.

\section{ENERGY TRANSFER TO THE MEDIUM}

\section{II.A. Collisional energy loss}

An electron of kinetic energy $T_{e}$ - will lose its energy in a medium through a large number of soft collisions or a few hard collisions with the atomic electrons of the absorber medium. The classical calculation of this collisional energy loss by charged ions, colliding with atomic electrons, was developed by $\mathrm{Bohr}^{7,8}$ and Bethe $\mathrm{e}^{9,10}$. When an ion of charge Ze and velocity $v$ interacts with an atomic electron, assumed to be free and at rest, there will be a transfer of momentum $\Delta p$ :

$$
\Delta p=\int_{-\infty}^{\infty} F_{c} d t \equiv \int_{-\infty}^{\infty} \frac{Z e^{2}}{\left(x^{2}+b^{2}\right)} \frac{d x}{v} \cong \int_{-\infty}^{\infty}\left(F_{c}\right)_{\perp} \frac{d x}{v}=\frac{2 Z e^{2}}{v b}
$$


with $F_{c}$ the Coulomb force between the negative electron of charge $e$ and the positive ion, which is at position $x$. Assuming that $b$ does not change, the parallel component of $F_{c}$ cancels out, and only the perpendicular component $\left(\left(F_{c}\right)_{\perp}=F_{c}\left(b /\left(x^{2}+b^{2}\right)^{1 / 2}\right)\right)$ is considered with $v$ a constant and $d t$ replaced by $d x / v$. The non-relativistic energy transferred to the atomic electron can then be written as:

$$
\Delta T_{e^{-}}=\frac{\Delta p^{2}}{2 m_{0}} \cong \frac{2 z^{2} e^{4}}{m_{0} v^{2} b^{2}}
$$

It is now interesting to take a sum over all the electrons at an impact parameter $b$. We let $n^{0}$ be the number of atoms of atomic number $z$ per unit volume. Therefore there are $n^{0} z$ electrons per unit volume, and ion travelling a distance $d x$ will encounter $2 \pi b d b d x n^{0} z$ electrons, and lose energy as follows:

$$
d T_{e^{-}\{b \rightarrow(b+d b)\}}=\Delta T_{e^{-2}} \cdot \pi b n^{0} z \cdot d b \cdot d x .
$$

The rate of energy loss per unit path length, $-d T / d x$, is found by integrating Eq. (3-3) over $b$ from a minimum $b_{\min }$ to a maximum $b_{\max }$ value:

$$
-\frac{d T}{d x}=\frac{4 \pi Z^{2} e^{4}}{m_{0} v^{2}} n^{0} z \int_{b_{\min }}^{b_{\max }} \frac{d b}{b}=\frac{4 \pi Z^{2} e^{4}}{m_{0} v^{2}} n^{0} z \ln \frac{b_{\max }}{b_{\min }} .
$$

The choice of $b_{\min }$ and $b_{\max }$ must be based on quantum-mechanical and relativistic considerations ${ }^{11}$. Here we are mainly concerned with orbital electrons and we will only consider electrons as incident particles, therefore, $Z$ will simply become 1 .

As the velocity of the incident particle approaches $c$, Eq. (3-4) should become constant. However, as illustrated in Fig. 3-2, the electron collisional stopping power, $(d T / d x)_{c}$, increases slightly at relativistic electron energies. This increase is a result of a contraction of the electric field, enabling distant interactions that will be more probable. From Eq. (3-2) we note that the amount of energy transferred varies as the inverse of the square of the impact parameter. In the case of electron-electron scattering, the maximum 
energy that can be transferred is given by $\Delta T_{e^{-}}=(\gamma-1) m_{o} c^{2}$, and with this energy value we can calculate $b_{\min }$ with the help of Eq. (3-2) as:

$$
b_{\min }=\frac{1}{\beta} \sqrt{\frac{2}{(\gamma-1)}} \frac{e^{2}}{m_{o} c^{2}},
$$

with $\gamma \equiv\left(1-\beta^{2}\right)^{-1 / 2}$, and $\beta=v / c$. The formulation of the collisional stopping power for electron and positron that accounts for the relativistic effect and the density effect, will be discussed in Section III.A

\section{II.B. Radiative energy loss}

An accelerated charged particle radiates energy as predicted by classical electromagnetism ${ }^{12}$. The Larmor equation, for a non-relativistic accelerated charge particle, states that the rate of energy loss $d E / d t$ is given by:

$$
\frac{d E}{d t}=\frac{e^{2} a^{2}}{6 \pi \varepsilon_{o} c^{3}}
$$

with $a$ the charged particle acceleration proportional to $z Z / M$, where $M$ is the mass of the accelerated charged particle, $z$ the charge of the particle, and $Z$ the atomic number of the nucleus. Therefore, the rate of energy loss to bremsstrahlung is proportional to the square of the product of the nuclear and incident particle charge divided by the mass of the charged particle:

$$
\frac{d E}{d t} \propto\left(\frac{z Z}{M}\right)^{2}
$$

Hence, the rate of energy loss to radiation should be more important for a light particle, such as an electron, passing through a high $Z$ absorber. This is illustrated in Fig. 3-2, where the larger the $Z$ of the absorber material, the larger is the radiative stopping power. We also see from this plot that the radiative energy loss is roughly proportional to the electron 
kinetic energy, and that the radiative contribution to the energy loss becomes important at energies above $10 \mathrm{MeV}$. The radiative loss becomes more important than collisional loss above electron kinetic energies of $10 \mathrm{MeV}$ for lead, $20 \mathrm{MeV}$ for copper, and $60 \mathrm{MeV}$ for water.

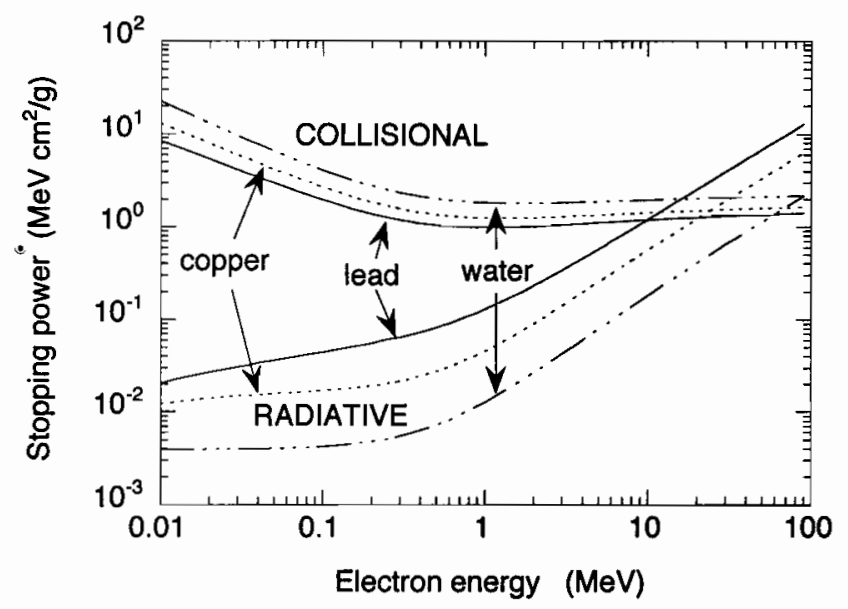

Figure 3-2. Plot of collisional and radiative electron mass stopping power in copper, lead, and water.

\section{ELECTRON STOPPING POWERS}

The average linear rate of energy loss of an electron in a medium (in $\mathrm{MeV} \mathrm{cm}$ ) is of primary importance in radiation physics and dosimetry. This quantity, called electron stopping power $S$ of the particular medium, is often normalized by the density of the medium, to obtain the mass stopping power $(\mathrm{S} / \rho)$, with units of $\mathrm{MeV} \mathrm{cm}^{2} \mathrm{~g}^{-1}$. It is convenient to separate stopping powers into two quantities: collisional stopping power $(S / \rho)_{c o l}$ and radiative stopping power $(S / \rho)_{\text {rad }}$, such that the total stopping power represents the sum of the two:

$$
\left(\frac{S}{\rho}\right)_{\text {total }}=\left(\frac{S}{\rho}\right)_{c o l}+\left(\frac{S}{\rho}\right)_{\text {rad }}
$$

The collisional stopping power corresponds to the rate of energy loss from soft and hard collisions, described in Section II.A, whereas the radiative stopping power is a result of energy loss from radiative interactions, discussed in Section II.B. It is important to differ- 
entiate between the two quantities, since their contribution to the absorbed dose in the medium will be different: energy given to collisions contributes along the area surrounding the ion track of the electron to the dose, whereas energy radiated is carried away from the point of interaction by the bremsstrahlung photons and does not contribute locally to the dose.

The accurate knowledge of stopping powers is very important for the determination of dose to the medium. Numerous authors have investigated this topic ${ }^{9-11,13,14}$. Report 37 of the International Commission on Radiation Units and Measurements ${ }^{15}$ reviews the most recent formulations of stopping power and values.

\section{III.A. Mass collisional stopping power}

Collisional mass stopping power can be defined as:

$$
\left(\frac{S}{\rho}\right)_{c o l}=\frac{N_{A} Z}{A} \int_{\mathrm{W}_{\min }}^{W_{\max }} W \frac{d \sigma}{d W} d W
$$

where $N_{A}$ is the Avogadro's number, $A$ the atomic mass of the medium in $\mathrm{g} / \mathrm{g}$-atom, $Z$ the atomic number of the medium, and $d \sigma / d W$ the differential electronic cross section for inelastic scattering (ionization or excitation). The result of the interaction is an energy loss $W$, between a minimum value $W_{\min }$ below which no excitation or ionization can happen, and a maximum possible value $W_{\max }$. It is also convenient to separate the soft and hard collisions in relationship with energy deposition location; the hard collisions create $\delta$ rays that may deposit their energy at a distance from their creation point. Therefore:

$$
\begin{aligned}
\left(\frac{S}{\rho}\right)_{c o l} & =\frac{N_{A} Z}{A}\left[\int_{0}^{W_{c}} W \frac{d \sigma}{d W} d W+\int_{W_{c}}^{W_{\max }} W \frac{d \sigma}{d W} d W\right] \\
& =\left(\frac{S}{\rho}\right)_{c o l}^{W<W_{c}}+\left(\frac{S}{\rho}\right)_{c o l}^{W_{c}>W_{c}},
\end{aligned}
$$

with $W_{c}$ an arbitrary energy cutoff value that separates soft and hard collisions, greater than the mean binding energy of the atomic electrons of the medium. 
The Bethe stopping power formula ${ }^{9,10,15}$ is widely used in medical physics. The collisional stopping power for electrons and positrons of energy $T$, in a medium of density $\rho$, is given by:

$$
\left(\frac{S}{\rho}\right)_{c o l}=\frac{2 \pi r_{e}^{2} m_{0} c^{2}}{\beta^{2}}\left(\frac{N_{A} Z}{A}\right)\left[\ln \left(T / I^{2}\right)+\ln (1+\tau / 2)+F^{ \pm}(\tau)-\delta-\frac{C}{Z}\right]
$$

with

$$
F^{-}(\tau)=\left(1-\beta^{2}\right)\left[1+\frac{\tau^{2}}{8}-(2 \tau+1) \ln 2\right]
$$

for electrons and

$$
F^{+}(\tau)=2 \ln 2-\left(\frac{\beta^{2}}{12}\right)\left[23+\frac{14}{(\tau+2)}+\frac{10}{(\tau+2)^{2}}+\frac{4}{(\tau+2)^{3}}\right]
$$

for positrons, where $\tau \equiv T /\left(m_{0} c^{2}\right), r_{0}$ the classical electron radius, $N_{A} Z / A$ the number of electrons per gram of medium, $I$ the mean excitation energy, $\delta$ the density-effect correction parameter and $C / Z$ the shell correction. The $F^{ \pm}$factor takes into account the difference of charge and spin between electrons and positrons. Basically, large energy losses are treated according to the Møller cross section for electrons, and to Bhabha cross sections for positrons ${ }^{16}$. For positrons, the maximum energy transferred to an atomic electron is greater than the energy that can be transferred from an incident electron because the incident and atomic electron are identical particles.

The mean excitation energy I corresponds to the mean value of all ionization and excitation energies of the atoms in the medium. In general, $I$ cannot be calculated from atomic theory to a useful degree of accuracy, and is determined experimentally. An extensive review of I-values is provided by Berger and Seltzer ${ }^{17}$.

The density effect parameter $\delta$ takes into account the polarization of the medium by the incident particles and it decreases the stopping power values, since the polarization decreases the Coulomb field near the incident particle track. The density effect increases with particle momentum, as relativistic effects make distant collisions more probable. This 
effect is negligible at low incident electron kinetic energies or in gases, where atoms are sufficiently separated, so that each atom is considered independent of the next. However, for high enough electron energies, the polarization effect becomes important, even for gases. The density effect can be computed from the knowledge of the dielectric response of the medium. Sternheimer provided a theory ${ }^{18}$ to calculate $\delta$ and he later devised a simple yet accurate fitting formula ${ }^{19}$ based on the numerical values of $\delta$ at various energies:

$$
\delta=\left(\begin{array}{ll}
4.6052 X+C, & X>X_{1} \\
4.6052 X+a\left(X_{1}+X\right)^{m}+C, & X_{0}<X<X_{1} \\
0 \text { for non-conducting materials, } & X<X_{0} \\
\delta\left(X_{0}\right) 10^{2\left(X-X_{0}\right)} \text { for conducting materials, }, & X<X_{0}
\end{array}\right.
$$

with $X \equiv \log _{10}(\tau(\tau+2))^{1 / 2}, C=-2 \ln \left((2 \pi I) /\left(h \omega_{p}\right)\right)$, $h \omega_{p} /(2 \pi)=28.816(\rho Z / A)^{1 / 2}$ the plasma energy in $\mathrm{eV}$ and $X_{0}, X_{1}, a, m$, and $C$ fitted parameters determined for 278 different materials. Figure 3-3 shows a plot of Sternheimer density effect for water, graphite, and polystyrene, as a function of incident electron energy ${ }^{15}$. As expected, the density effect increases with electron energy and with the density of the medium.

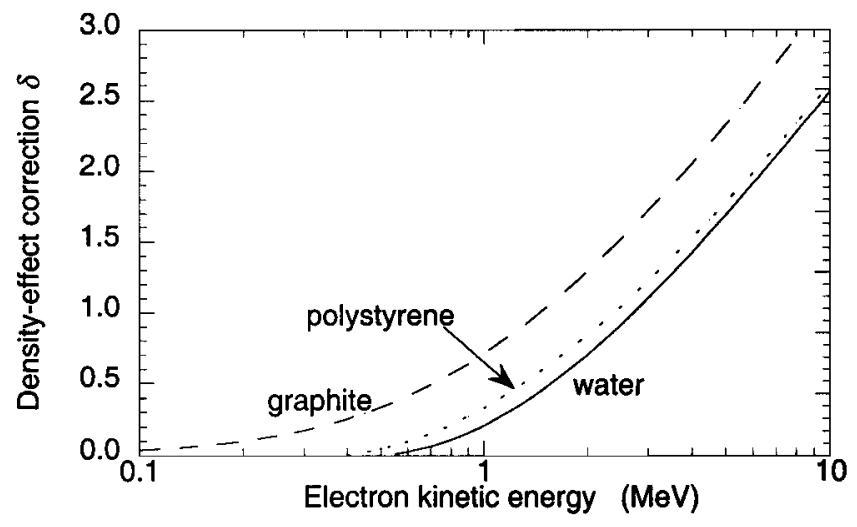

Figure 3-3. Sternheimer correction factor $\delta$ for density-effect for electrons in graphite, polystyrene and water as a function of incident electron energy. 
The shell correction parameter $C / Z$ is used to correct for loss of interactions with the lower shells, $K$ or $L$, for which the assumption that the velocity of the target electron is much lower than that of the incident electron ${ }^{6}$ does not hold. As the energy of the incoming electron is decreased, its probability of interaction with the lower shells decreases and the stopping power is reduced.

\section{III.B. Mass radiative stopping power}

The mass radiative stopping power $(S / \rho)_{\text {rad }}$ gives the rate in $\mathrm{MeVcm}^{2} \mathrm{~g}^{-1}$ of bremsstrahlung production by electrons or positrons, and is given by:

$$
\left(\frac{S}{\rho}\right)_{r a d}=\sigma_{0} \frac{N_{A} Z^{2}}{A}\left(T+m_{0} c^{2}\right) \bar{B}_{r}
$$

with $\sigma_{0}=(1 / 137)\left(e^{2} /\left(m_{0} c^{2}\right)\right), T$ the kinetic energy of the incident electron or positron, and $\bar{B}_{r}$ a function of $Z$ and $T\left(\bar{B}_{r}\right.$ is $16 / 3$ for $T \ll 0.5 \mathrm{MeV}, 6$ for $T=1 \mathrm{MeV}, 12$ for 10 $\mathrm{MeV}$ and 15 for $100 \mathrm{MeV}$ (Ref. \#O). Extensive tables of radiative stopping powers are given by Berger and Seltzer ${ }^{17}$.

\section{RESTRICTED MASS STOPPING POWER}

Collisional stopping power can be divided into a soft and a hard collision component as indicated in Eq. (3-10). If the higher energy cutoff $\Delta$ of the sum of the two integrals is $W_{\max }$, the stopping power $\left(\frac{L}{\rho}\right)_{\infty}$ is called the unrestricted collisional stopping power:

$$
\left(\frac{S}{\rho}\right)_{c o l}=\left(\frac{L}{\rho}\right)_{\Delta=W_{\max }}=\left(\frac{L}{\rho}\right)_{\infty}
$$


Calculating dose to the medium (as described in Chapter 4) with the unrestricted collisional stopping power may produce an overestimate of the dose, if the region of interest for the calculation is small. As we have mentioned previously, some $\delta$ rays produced by hard collisions do not deposit their energy locally. In the region of interest we must have a $\delta$-ray equilibrium, so that for each $\delta$ ray escaping the region, another one created in another region enters that first region. It is useful to define a restricted stopping power, $(L / \rho)_{\Delta}$, that includes all soft collisions and the hard collisions that produce secondary electrons having a kinetic energy less than some cutoff value $\Delta$. Therefore, the choice of the cutoff value is based on the geometry of the region of interest such that the energy of the cutoff corresponds to the energy of an electron having a mean range equal to the size of the region.

The restricted mass stopping power for electrons and positrons is given by ${ }^{15}$ :

$$
\left(\frac{L}{\rho}\right)_{\Delta}=\frac{2 \pi r_{e}^{2} m_{0} c^{2}}{\beta^{2}}\left(\frac{N_{A} Z}{A}\right)\left[\ln \left(T / I^{2}\right)+\ln (1+\tau / 2)+\mathrm{G}^{ \pm}(\tau, \eta)-\delta\right]
$$

This expression is the same as Eq. (3-11) but the $G$ factors are given by:

$$
\begin{aligned}
G^{-}(\tau, \eta) & =-1-\beta^{2}+\ln (4(1-\eta) \eta)+\frac{1}{(1-\eta)} \\
& +\left(1-\beta^{2}\right)\left[\frac{t^{2} \eta^{2}}{2}+(2 \tau+1) \ln (1-\eta)\right]
\end{aligned}
$$

for electrons and by:

$$
\begin{aligned}
G^{+}(\tau, \eta)=\ln 4 \eta-\beta^{2} & \cdot\left[1+\left(2-\xi^{2}\right) \eta-\left(3+\xi^{2}\right)\left(\frac{\xi \tau}{2}\right) \eta^{2}\right. \\
& \left.+(1+\xi \tau)\left(\frac{\xi^{2} \tau^{2}}{3}\right) \eta^{3}-\left(\frac{\xi^{3} \tau^{3}}{3}\right) \eta^{4}\right]
\end{aligned}
$$

for positrons with $\xi=(\tau+2)^{-1}, G^{-}(\tau, 1 / 2)=F^{-}(\tau)$, and $G^{+}(\tau, 1)=F^{+}(\tau)$. 


\section{RAdiation yield}

The total energy fraction of the initial kinetic energy of the incident electron that is transferred to radiation by bremsstrahlung is called the radiation yield $Y(T)$. At a given energy $T$, the instantaneous radiation yield $y(T)$ is given by:

$$
y(T)=\frac{(S / \rho)_{\text {rad }}}{(S / \rho)_{t o t}} .
$$

The radiation yield $Y\left(T_{0}\right)$ for an electron of initial energy $T_{0}$ is calculated by:

$$
Y\left(T_{0}\right)=\frac{\int_{T_{0}}^{Q} y(T) d T}{\int_{T_{0}} d T}=\frac{1}{T_{0}} \int_{0}^{T_{0}} y(T) d T .
$$

The $g$ factor of Eq. (2-32) is an average of $Y\left(T_{0}\right)$ for all the initial energies $T_{0}$ of the electrons and positrons present in a radiation beam. Therefore $\mathrm{g}$ is given by:

$$
g=\frac{\int_{0}^{T_{\max }} \Phi_{T} Y\left(T_{0}\right) d T}{\int_{T_{0}}^{T_{\text {max }}} \Phi_{T} \mathrm{~d} T}
$$

with $\Phi_{T}$ the total secondary electron fluence spectrum in the medium produced by a photon beam.

\section{STOPPING POWER RATIOS}

For ionization chamber dosimetry, it is useful to define collisional mass stopping power ratios as: 


$$
\frac{\left(\frac{S}{\rho}\right)_{\text {medium 1 }}}{\left(\frac{S}{\rho}\right)_{\text {medium 2 }}}=\left(\frac{S}{\rho}\right)_{\text {medium 2 }}^{\text {medium 1 }}
$$

This quantity is of primary importance in the Bragg-Gray and Spencer-Attix cavity theories for the determination of the $\operatorname{dose}^{20}$ (see Chapter 4). The Spencer-Attix theory ${ }^{21}$, as formulated by Nahum ${ }^{22}$, takes into account the cutoff energy, discussed previously, and proposes a dose equation for which the mean restricted mass stopping power ratio is given by:

$$
\left(\frac{S}{\rho}\right)_{\mathrm{b}}^{\mathrm{a}}=\frac{\int_{\int_{\max }}^{T_{\max }} \Phi_{T}\left(\frac{L}{\rho}\right)_{\Delta, \mathrm{a}} \mathrm{d} T+\left(\Phi_{T, \mathrm{a}}(\Delta)\right)\left(\frac{S(\Delta)}{\rho}\right)_{\mathrm{a}}}{\int_{\Delta}^{\max } \Phi_{T, \mathrm{a}}\left(\frac{L}{\rho}\right)_{\Delta, \mathrm{b}} \mathrm{d} T+\left(\Phi_{T, \mathrm{a}}(\Delta)\right)\left(\frac{S(\Delta)}{\rho}\right)_{\mathrm{b}}},
$$

where $\Phi_{T}$ is the total electron fluence spectrum, $(S(\Delta) / \rho)$ is the unrestricted stopping power evaluated at energy $\Delta$, and $\Phi_{T, \mathrm{a}}(\Delta)$ is the total electron fluence spectrum also evaluated at the threshold energy $\Delta$.

\section{STOPPING POWER OF COMPOUNDS}

The various stopping power types in mixtures, elements or chemical compounds are approximated through the Bragg's additive rule ${ }^{15}$ which assumes that each atom contributes almost independently to the stopping power, resulting in the stopping power for the mixture simply given by a weighted addition:

$$
\left(\frac{S}{\rho}\right)_{\text {mix }}=w_{Z_{1}}\left(\frac{S}{\rho}\right)+w_{Z_{2}}\left(\frac{S}{\rho}\right)+\ldots
$$

where $w_{Z j}$ is the weight fraction of an element of atomic number $Z_{j}$. Density correction $\delta$ and mean ionization energy $I$ can also be obtained with the same rule. 


\section{ENERGY ABSORBED BY THE MEDIUM}

The dose absorbed by the medium is the amount of energy absorbed by unit mass of medium in units of Gray (Gy), with $1 \mathrm{~Gy} \equiv 1 \mathrm{~J} / \mathrm{kg}$, see Chapter 4 Section I.C.

For a monoenergetic electron beam of initial energy $T$, passing across a thin slab of medium, the absorbed dose $D$ to the medium will be given by:

$$
D=1.602 \times 10^{-10} \Phi_{T}\left(\frac{L}{\rho}\right)_{\Delta}^{T} \quad \text { (in Gy) }
$$

with $1.602 \times 10^{-10}$ the conversion from $\mathrm{MeV} / \mathrm{g}$ to $\mathrm{Gy}, \Phi_{T}$ the monoenergetic electron fluence $\left(\mathrm{cm}^{-2}\right)$, and $(L / \rho)_{\Delta}^{T}$, the mass restricted collisional stopping power ratio in $\left(\mathrm{MeV} \cdot \mathrm{cm}^{2} \cdot \mathrm{g}^{-1}\right)$. For Eq. (3-26) to be valid, it is assumed that the collisional stopping power remains constant and is only a function of the initial electron energy $T$. Also, charged particle equilibrium of $\delta$-rays must exist and radiative photons should not contribute to the dose, and finally scattering must be negligible. The last assumption is the weakest for an electron beam, and in practice, the dose equation has to be corrected to account for that. Generally, radiotherapy beams are not monoenergetic, and the dose has to be calculated from a polyenergetic spectrum of electrons $\Phi_{e}(T)\left(\mathrm{MeV}^{-1} \mathrm{~cm}^{-2}\right)$. Integrating Eq. (3-26) over all electron energies yields:

$$
D=1.602 \times 10^{-10} \int_{0}^{\mathrm{T}_{\max }} \Phi_{\mathrm{e}}(T)\left(\frac{L(T)}{\rho}\right)_{\Delta} \mathrm{d} T=1.602 \times 10^{-10} \Phi_{\mathrm{e}}\left(\frac{\bar{L}}{\rho}\right)_{\Delta}
$$

with $(\bar{L} / \rho)_{\Delta}$, the mean restricted stopping power.

\section{SUMmaRY}

There are three different methods by which electrons and positrons interact with the medium: soft collisions $(b » a)$, hard collisions $(b \sim a)$, and radiative collisions $(b \ll a)$ with $a$ the mean atomic radius and $b$ the classical impact parameter. Soft collisions are the 
most probable interactions but each soft collision interaction transfers only a small amount of energy to the medium and involves electron Coulomb-force interactions between the incident particle and the atomic electron cloud as a whole. Hard collisions are less probable but each one results in a much greater energy transfer and involves Coulomb-force interactions between the incident particle and an individual orbital electron. Over all, a comparable amount of energy is lost by all soft and hard collisions. For small impact parameters ( $b$ «a) the electron will interact with a nucleus. Most of these interactions, about 98\%, scatter the electron elastically; the remaining $2 \%$ give rise to emission of photons (bremsstrahlung).

Multiple scattering theories are used to estimate deflections and energy loss by the electrons and positrons. Bethe's formula, with various correction factors, is used to estimate stopping power as the rate of kinetic energy loss per unit pathlength of the particle. Total stopping power is divided into a collisional and a radiative part. Energy loss to collisions contributes locally to the dose, except for $\delta$ rays which may carry energy far from the interaction location. To account for this local loss of energy, a restricted stopping power is defined with a cutoff energy $\Delta$, above which the energy is considered to be transported away by $\delta$ rays. The choice of the cutoff is based on the dimensions of an ionization chamber sensitive volume.

The dose is defined as the amount of energy absorbed by the medium per unit volume. It can be calculated by the product of the stopping power and electron fluence for a monoenergetic beam. However, in general, for a polyenergetic beams, it should be a sum over each electron energy of the spectrum to obtain the total dose absorbed locally. 


\section{References}

1 G. Z. Molière, "Theorie der Streuung schneller geladener Teilchen I: Einzelstreuung am abgeschirmten Coulomb-Feld," Z. Naturforsch 2a, 133 (1947).

2 G. Z. Molière, "Theorie der Steuung schneller geladener Teilchen. II. Mehrfach-und Vielfachstreuung," Z. Naturforsch 3a, 97 (1948).

3 N. F. Mott, "The Scattering of Fast Electrons by Atomic Nuclei," Proc. Roy. Soc. A. A124, 426 (1929).

4 S. Goudsmit and J. L. Saunderson, "Multiple Scattering of Electrons," Phys. Rev. 57, 24-29 (1940).

5 S. Goudsmit and J. L. Saunderson, "Multiple Scattering of Electrons II," Phys. Rev. 58, 36-42 (1940).

6 Frank H. Attix, Introduction to radiological physics and radiation dosimetry (Wiley \& Son, New York, 1986).

7 N. Bohr, "On the Theory of the Decrease of Velocity of Moving Electrified Particles on Passing through Matter," Phil. Mag. 25, 10 (1913).

8 N. Bohr, "Kgl. Danske Videnskab. Selskab," Mat.-Fys. Medd. 18 (8) (1948).

9 H. Bethe and W. Heitler, "On the stopping of fast particles and on the creation of positive electrons," Proc. Roy. Soc. A. 146, 83-112 (1934).

10 H. A. Bethe, "Zur Theorie des Durchgangs schneller Korpuskularstrahlen durch Materie," Ann. Physik 5, 325 (1930).

11 Pierre Marmier and Eric Sheldon, Physics of nuclei and particles (Academic Press, New York, 1969). 
12 John David Jackson, Classical Electrodynamics , 3rd ed. (John Whiley, New York, 1999).

13 M. S. Livingston and H. A. Bethe, "Nuclear physics," Rev. Mod. Phys. 9, 245 (1937).

14 E. J. Williams, "The passage of $\alpha$ and $\beta$ particles through matter and Born's Theory of collisions," Proc. Roy. Soc. A. 135, 108-142 (1932).

15 ICRU, "Stopping powers for electrons and positrons," ICRU Report 37, International Commission on Radiation Units and Measurements, Bethesda, Maryland (1984).

16 M. J. Berger and R. Wang, "Multiple-Scattering Angular Deflections and Energy-Loss Straggling.," in Monte Carlo Transport of Electrons and Photons, edited by Theodore M. Jenkins, Walter R. Nelson and Alessandro Rindi (New York, 1988), pp. 21-56.

17 M. J. Berger and S. M. Seltzer, "Stopping powers and ranges of electrons and positrons," Report NBSIR 82-2550-A, National Bureau of Standards, Washington D. C. (1983).

18 R. M. Sternheimer, "The Density Effect for the Ionization Loss in Various Materials," Phys. Rev. 88, 851-859 (1952).

19 R. M. Sternheimer, "Density Effect for the Ionization Loss of Charged Particles in Various Substances," Atom. Data and Nucl. Data Tables 30, 261 (1984).

20 F. H. Attix, "A simple derivation of Ngas, a correction in Awall, and other comments on the AAPM Task Group 21 protocol," Med. Phys. 11 (5), 725-728 (1984).

21 L. V. Spencer and F. H. Attix, "A theory of cavity ionization," Radiat. Res. 3, 239-254 (1955).

22 A. E. Nahum, "Water/Air Mass Stopping Power Ratios for Megavoltage Photon and Electron Beams," Phys. Med. Biol. 23, 24 (1978). 


\section{Chapter 4}

\section{Measurement of absorbed dose}

In radiation oncology, accurate determination of the dose delivered to a tumor and healthy surrounding tissue is of primary importance for the outcome of a treatment. Practical clinical dosimetry today is based on the absorbed dose, and accurate measurement of absorbed dose represents one of the major responsibilities of clinical medical physicists. Information about the optimal tumour dose for treating various cancers is based on medical experience gained during the past century from around the world. Therefore, sophisticated international radiation dosimetry protocols are in place with the goal of ensuring accurate and uniform dose measurement. However, these protocols present a restricted approach, since they only define measurement of the dose under a specified set of reference conditions; the dose to water delivered by accelerator or cobalt- 60 beams incident on a water phantom.

To understand the basis of the radiation dosimetry protocols, a review of the quantities used to characterize a radiation beam as well as a description of the mechanisms in which energy is transferred and then absorbed by the medium is given below.

\section{BASIC QUANTITIES USED IN RADIATION DOSIMETRY}

\section{I.A. Fluence}

For a monoenergetic photon beam, the particle fluence $\phi$ is defined as the number of photons $d N$ intersecting a sphere of cross sectional area $d A$ per unit time ${ }^{1}$ : 


$$
\phi=\frac{d N}{d A}
$$

with units of $\mathrm{m}^{-2}$ and the geometry illustrated in Fig. 4-1. Particle fluence is constant even if the beam has an oblique incidence, whereas the planar fluence, defined as the number of particles crossing a plane per unit area, decreases with oblique incidence of the beam.

Most clinical photon beams are composed of photons of various energies, and the fluence becomes a function of the photon energies $h v$ :

$$
\phi(h v)=\frac{d N(h v)}{d A}
$$

(a)

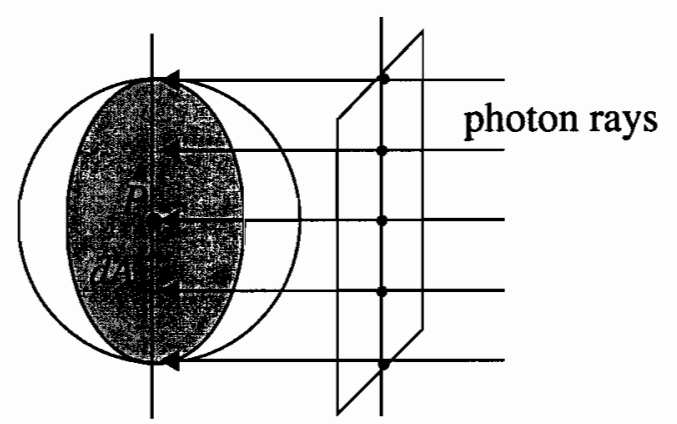

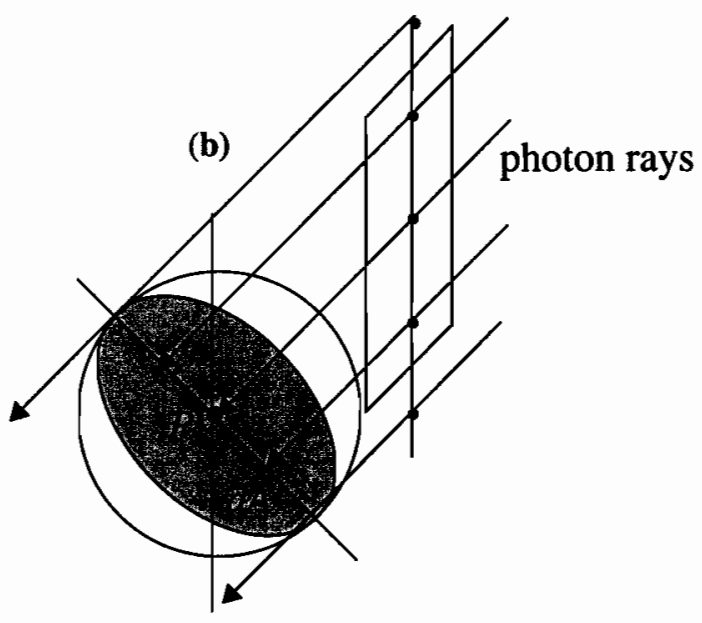

Figure 4-1. Radiation photon field incident on a sphere of cross sectional area $d A$ around point $P$ for (a) horizontal incidence, and (b) oblique incidence. Particle fluence is the same in (a) and (b), however, the planar fluence decreases for oblique incidence.

A photon beam can also be characterized by the photon energy fluence $\psi$ which relates the amount of energy carried by the photon beam per cross-sectional area $\mathrm{d} A$ :

$$
\psi=\frac{d N(h v) \cdot h v}{d A}
$$

The distribution which gives the number of photons that have a given energy is called an energy spectrum. Characterization of a radiation beam in terms of fluence is the basis of the 
Bragg-Gray cavity theory, discussed in Section II.A. However, experimental knowledge of the exact beam fluence or spectrum is extremely difficult to obtain and Monte Carlo techniques are often used to calculate the fluence and spectrum of a radiation beam.

\section{I.B. Kinetic energy released in medium}

The kerma $K$ (kinetic energy released in medium) is a quantity relevant to indirectly ionizing radiations, such as photons which transfer their energy to the absorbing medium in a two step process. The first step consists of the interaction of the photons with the absorber medium, discussed in Chapter 1 , which results in a transfer of energy to electrons that are set in motion in the medium. Kerma is expressed as the mean energy transferred, $d \bar{E}_{t r}$, given by Eq. (2-31), per mass element $d m$ of the medium as defined by ICRU $^{1}$ :

$$
K=\frac{d \bar{E}_{t r}}{d m}
$$

with units of $\mathrm{J} / \mathrm{kg}$. The second step, discussed in Chapter 3, is the actual energy transfer from the electron to the medium through excitation and ionization of atoms composing the medium.

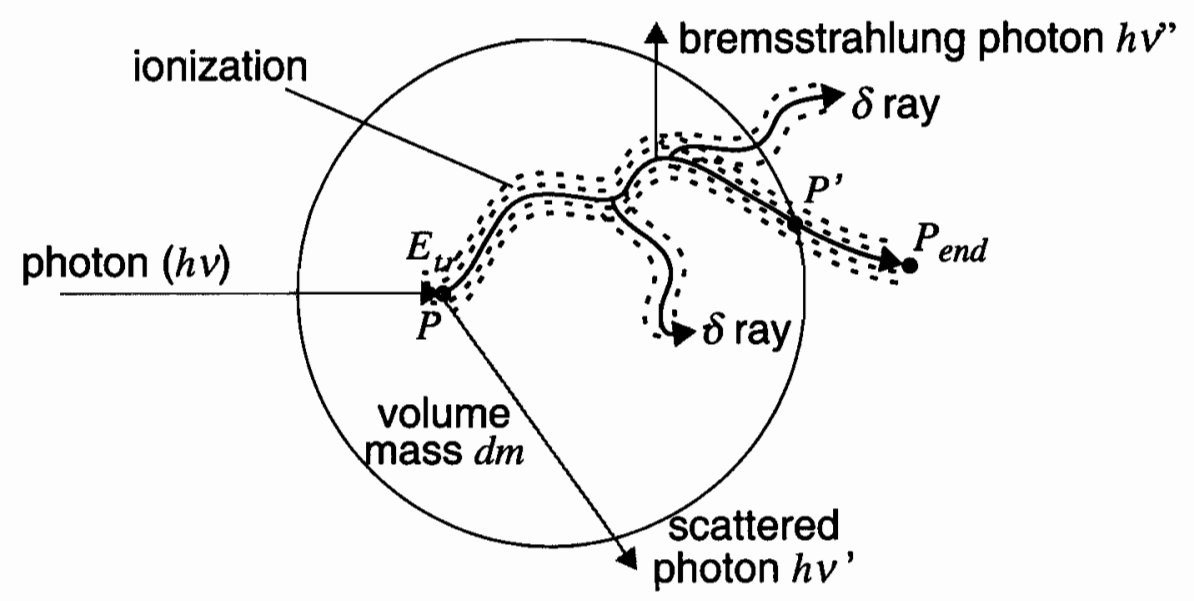

Figure 4-2. Transfer of energy from a photon to the medium. The photon interacts at point $P$, and transfers some of its energy in the form of electron's kinetic energy. The electron, in turn, gives its energy to the medium through small collisions along its track, from $P$ to $P_{\text {end }}(R e f . \# 3)$. One of the $\delta$ rays carries its energy out of the volume mass $d m$. 
Kerma is the quantity that relates most explicitly the description of the radiation and its effects. Kerma can also be expressed as a function of the photon fluence as:

$$
K=\phi\left(\frac{\mu}{\rho}\right) \bar{E}_{t r}
$$

where $(\mu / \rho)$ is the photon mass attenuation coefficient of the medium. The product $\phi(\mu / \rho)$ yields the number of photon interactions per unit mass of material. The kerma must always be defined with respect to the specific material in which the interactions are taking place (e.g., air-kerma, water kerma, etc.). For realistic beams the photon fluence is composed of a spectrum of photon energies. Integrating over the energy range of the photons, from 0 to a maximum value $h v_{\max }$, the kerma becomes:

$$
K=\int_{h v=0}^{h v=h v_{\max }} \frac{d \phi(h v)}{d h v}\left(\frac{\mu(h v)}{\rho}\right) \bar{E}_{t r}(h v) \cdot d h v .
$$

As mentioned in Chapter 3, once the electron is set in motion in the medium, it can lose energy through collisional interactions (excitation, ionization, $\delta$ ray production) and radiative bremsstrahlung interactions. Therefore, the kerma can be subdivided into a radiative and a collisional component. This distinction is important when relating the kerma to the absorbed dose.

\section{I.C. Absorbed dose}

The absorbed dose is the most important quantity in radiation dosimetry, defined by the ICRU ${ }^{1}$ as:

$$
D=\frac{d \bar{E}_{a b}}{d m}
$$

where $\bar{E}_{a b}$ is the mean energy absorbed, as defined in Eq. (2-33), in mass element $d m$ from ionizing radiation. The size of $d m$ has to be small enough to represent the dose value at a point, however, not too small to avoid significant statistical fluctuations from the energy 
deposition process. It has the same basic units as the kerma, $\mathrm{J} / \mathrm{kg}$, however, the special SI unit for dose is the gray (Gy), with $1 \mathrm{~Gy}=1 \mathrm{~J} / \mathrm{kg}$.

Dose and kerma are very similar quantities. They both express a quantity of energy per unit mass. The difference between the two comes from where this energy is deposited within the medium. For kerma, it does not matter whether the charged particles slow down inside the volume of interest or not; only the energy transferred to charged particles in the volume of interest is important. For the absorbed dose, on the other hand, only the energy deposited in the medium by the secondary electrons within the volume of interest is considered.

In Fig. 4-2, a photon of initial energy $h v$ impinges onto a volume of mass $d m$. A Compton interaction occurs and a scattered photon of energy $h v^{\prime}$ escapes the volume. The Compton electron with kinetic energy $E_{t r}=h v-h v$ ' deposits some of its energy along the path $P$ to $P_{\text {end }}$ through collisions. A bremsstrahlung photon $\left(h v^{\prime \prime}\right)$ is emitted by this electron and escapes the volume. A $\delta$ ray electron is also produced and this electron deposits its energy along its track within the volume of mass $d m$. The kerma for this situation is simply $E_{t r} / d m$. However, the dose to the volume is calculated considering only the energy given to the medium by the secondary electron along the path $P$ to $P^{\prime}$, excluding all energy that escapes the volume, i.e., excluding the energy of the Compton photon ( $\left.h v^{\prime}\right)$, the energy of the bremsstrahlung photon ( $\left.h v^{\prime \prime}\right)$, the energy lost by the electron along the $P^{\prime}$ to $P_{\text {end }}$ track and part of the energy of the second $\delta$ ray which escapes the volume $d m$.

For volumes which are large compared to the tracklength, the collisional kerma and absorbed dose are virtually identical. Since absorbed dose also includes energy deposition within the volume by electrons set in motion outside that volume this tends to balance the energy deposited outside the volume by those electrons starting inside the volume, which is the requirement for electronic equilibrium.

\section{I.D. Charged particle equilibrium (CPE)}

The concept of charged particle equilibrium (CPE) has important implications in radiation therapy. Since kerma and absorbed dose do not occur at the same point, a similar 
relation to Eq. (4-6) cannot be applied to calculate the absorbed dose unless electronic equilibrium exists at the point of calculation. For electronic equilibrium to exist, the number of secondary electrons entering and stopping inside the volume of interest must equal to the number of secondary electrons created within that volume and escaping that same volume. This is illustrated in Fig. 4-3, for two different cases without, (a), and with, (b), attenuation of the photon beam with depth. In (a), the number of electrons set in motion in each voxel is the same and the average energy given to the electron is the same and at depth of electronic equilibrium, the number of electrons stopping is equal to the number of electrons set into motion in a given voxel. Therefore, the dose equals the collisional kerma beyond the build up region.

The buildup region can be explained by the fact that the electrons set in motion near the surface deposit their energy deeper into the medium, therefore, the dose only reaches a plateau in the electronic equilibrium region. If bremsstrahlung photons are neglected, the dose is exactly equal to the total kerma, but if they are considered and attenuation of the photon beam is neglected the dose can be expressed in terms of kerma as follows:

$$
D=\phi\left(\frac{\mu}{\rho}\right) \bar{E}_{a b}=K(1-g)=K_{c o l}
$$

where $\bar{E}_{a b}$ is the mean energy absorbed per photon interaction, and $g$ is the fraction of electron's energy lost via radiative processes, as defined in Chapter 2, Section IV.H., and $K_{c o l}$ is the collisional kerma which does not contain contribution of bremsstrahlung photons.

In (b), exponential attenuation of the photon beam is accounted for. Under that condition, a strict electronic equilibrium is never attained, since the photon beam is attenuated and lower and lower number of electrons is set into motion with depth. Therefore, the kerma will decrease continuously with depth. The absorbed dose, on the other hand, will first increase in the build up region to reach a point of dose maximum at depth $d_{\max }$. At a slightly greater depth, equal to the range of the secondary particles created at the surface of the medium, the dose and kerma curves become parallel and both decrease exponentially, under a transient electronic equilibrium condition, separated by a nearly constant factor $\beta$. 
(a)

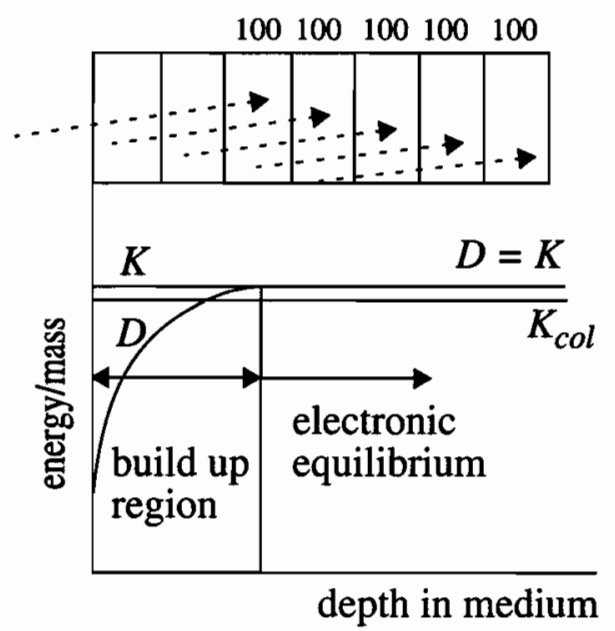

(b)

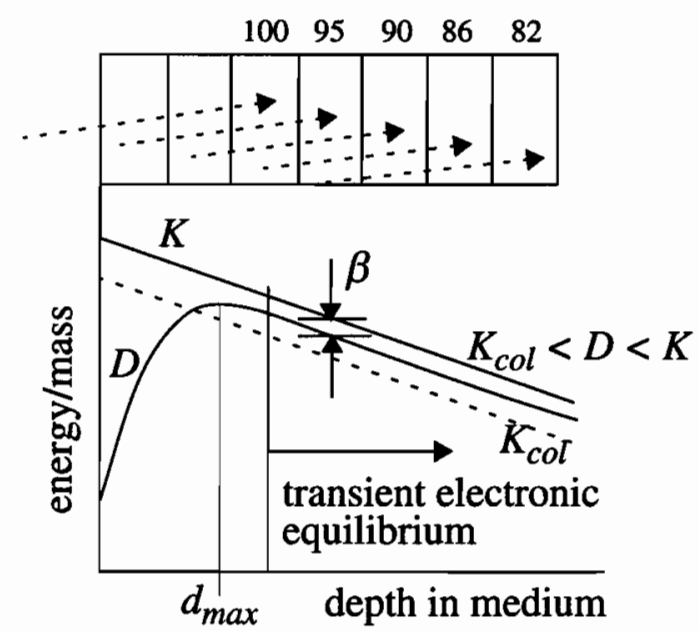

Figure 4-3. Illustration of the difference between the kerma and the absorbed dose, without (a) and with (b) attenuation of the photon beam ${ }^{2}$. In (a), the kerma is equal to the dose for depths deeper than the depth where electronic equilibrium is reached, whereas in (b) there never is a strict equilibrium and the kerma is always greater than the dose. Collisional kerma is less than total kerma because of energy escaping the volume of interest as bremsstrahlung photons.

Under the condition of transient electronic equilibrium the dose and the kerma are related by the following expression:

$$
D(d)=\phi(d)\left(\frac{\mu}{\rho}\right) \bar{E}_{a b} \beta=K(d) \beta(1-g)=K_{c o l}(d) \beta
$$

\section{Cavity theory}

When using a dosimeter to measure the dose in a medium, the dosimeter yields a reading that is related to the dose absorbed in its own medium. Cavity theories were developed to determine the dose to medium from the signal read by the dosimeter embedded in a phantom material. The most common dosimeter for which cavity theory is applied is the air-filled ionization chamber. The chamber is usually inserted into a dosimetric phantom in order to determine the absorbed dose from an ionization measurement. The presence of the air cavity of the chamber perturbs the dose measurement and must hence be 
accounted for. Many parameters have to be accounted for, such as the energy spectrum and angular distribution of the particles in the medium as well as the dosimeter size and its composition. Cavity theory is used to convert the absorbed dose measure in the dosimeter material to the absorbed dose given to the medium in which the dosimeter is embedded.

Assuming that all radiative photons escape the volume of interest and that secondary electrons are absorbed close to their creation point, similarly to equation Eq. (3-27), the dose $D_{m}$ to the medium $m$ is related to the fluence $\Phi_{\mathrm{T}}$ of the primary electrons of kinetic energy $T$ as:

$$
D_{m}=\Phi_{T}\left(\frac{S_{\mathrm{T}}}{\rho}\right)_{\mathrm{col}}^{m}
$$

where $\left(S_{\mathrm{T}} / \rho\right)_{\text {col }}^{m}$ is the unrestricted collisional stopping power for the medium. This relation assumes that all electrons have the same kinetic energy. If the electron beam passes through an interface between two media ( $c$ and $m$ ), as illustrated in Fig. 4-4, the ratio of the doses on each side of the interface is given by:

$$
\frac{D_{\mathrm{c}}}{D_{m}}=\frac{\left(S_{T} / \rho\right)_{c o l}^{c}}{\left(S_{T} / \rho\right)_{c o l}^{m}}=\left(\frac{S}{\rho}\right)_{m}^{c}
$$

with $(S / \rho)_{m}^{\mathrm{c}}$ the ratio of mass collisional stopping powers in media $c$ and $m$ for electrons of kinetic energy $T$. This relation assumes that the fluence $\Phi_{\mathrm{T}}$ is not modified by the interface and that backscatter radiation can be neglected.

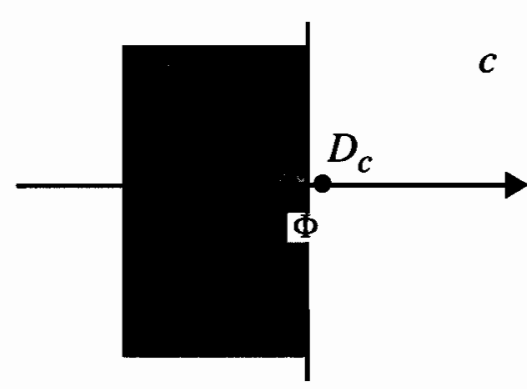

Figure 4-4. An electron beam going through an interface between medium $m$ and $c$ of the interface. The fluence is assumed to be identical in the two media, therefore, the absorbed doses on each side are related through Eq. (4-11). 
For a spectrum of electron energies, $\left(S_{\mathrm{T}} / \rho\right)_{\mathrm{col}}^{m}$ is replaced by the average mass collision stopping power given by:

$$
\left(\frac{\bar{S}}{\rho}\right)_{\mathrm{col}}^{m}=\frac{\int_{0}^{\mathrm{T}_{\max }} \Phi(T)(S(T) / \rho)_{\mathrm{col}}^{m} d T}{\int_{0}^{\mathrm{T}_{\max }} \Phi(T) d T}=\frac{D}{\Phi}
$$

where $\Phi(T) d T$ is the fluence of electrons with energies between $T$ and $T+d T$ and a ratio similar to that of Eq. (4-11) can be taken to relate the doses in $c$ and $m$.

\section{II.A. Bragg-Gray cavity theory}

W. H. Bragg ${ }^{4}$ and L. H. Gray ${ }^{5}$ developed the Bragg-Gray cavity theory as follows. Consider a homogeneous medium $m$ which contains a thin cavity filled with a medium $c$, as illustrated in Fig. 4-5.

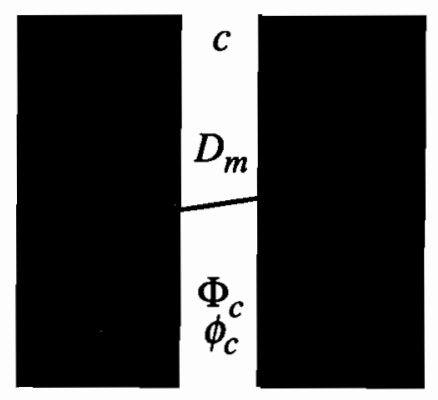

Figure 4-5. A cavity filled with medium $c$ within a homogeneous medium $m$.

For the Bragg-Gray theory to hold two conditions must be respected:

- first Bragg-Gray condition: the thickness of the cavity $c$ is assumed to be small in comparison with the range of charged particles incident on the cavity, such that the presence of the cavity does not perturb the charged-particle fluence in the medium $m$, i.e., $\Phi_{m}(T)=\Phi_{c}(T)$. In addition, the photon fluence $\phi$ is not disturbed by the presence of the cavity and therefore the scattering properties of the medium $c$ must be similar to those of medium $m$. 
- second Bragg-Gray condition: the absorbed dose in the cavity is assumed to be deposited entirely by the charged particles crossing it. Therefore the electrons crossing the cavity originate outside the cavity and no electrons are stopped within the cavity. In addition, no photon interactions occur inside the cavity.

When the two Bragg-Gray conditions hold, the electron fluence $\Phi(T)$ will not change across the cavity and similarly to Eq. (4-11) the ratio of the dose to medium $m$ and dose to medium $c$ can be expressed as:

$$
\frac{D_{m}}{D_{\mathrm{c}}}=\frac{(\bar{S} / \rho)_{\mathrm{col}}^{m}}{(\bar{S} / \rho)_{\mathrm{col}}^{\mathrm{c}}}=(\overline{\bar{S}})_{\mathrm{c}}^{m}
$$

where the unrestricted collisional stopping powers are averaged over the spectrum of primary electron energies. The theory also requires that a charged-particle equilibrium or transient charged particle equilibrium exists in the cavity.

For an air ionization chamber, the cavity is open to ambient air. The dose absorbed by the air cavity $D_{c}$ can be determined by measuring the charge $Q$ produced in the gas using the following relationship:

$$
D_{\mathrm{c}}=\frac{Q}{m_{\mathrm{c}}} \bar{W}_{\text {air }}
$$

where $\bar{W}_{\text {air }}$ is $33.97 \mathrm{~J} / \mathrm{C}$, as discussed in Chapter 1 , and $m_{c}$ is the mass in $\mathrm{kg}$ of the air in the cavity in which the charge $Q$ in coulombs was produced. Under Bragg-Gray conditions, Eq. (4-14) can be substituted in to Eq. (4-13) to yield the dose to the medium in the immediate vicinity of the cavity:

$$
D_{m e d}=\frac{Q}{m_{\mathrm{c}}} \bar{W}_{a i r}\left(\frac{\bar{S}}{\rho}\right)_{g}^{m}
$$

For a given cavity size, the requirements for Bragg-Gray cavity to hold will vary with radiation energy, however, the Bragg-Gray cavity theory does not take into account the size of the cavity. 


\section{II.B. Electron spectra in medium}

As mentioned previously, the calculation of electron or photon fluence spectra at each point within a medium is a very complex task, since it implies solving the Bolzmann transport equation which expresses the conservation of energy throughout the medium and in general no mathematical solutions are possible.

\section{II.C. CSDA electron fluence}

Consider a uniform mono-energetic electron fluence with energy $T_{0}$ within a medium. If charged particle equilibrium exists and bremsstrahlung radiation is neglected, the dose deposited by $N$ charged particles of energy $T_{0}$ can be stated as:

$$
D_{m}=N T_{0}
$$

The dose can also be expressed by a similar expression to the integral form of Eq. (3-27) and can be equated to the dose from Eq. (4-16) to give:

$$
D_{m}=\int_{0}^{T_{o}} \Phi_{T}^{m}(T) \frac{S_{m}(T)}{\rho} d T=N T_{0}
$$

Differentiating Eq. (4-17) with respect to $T_{0}$ yields for $\left(T \leq T_{0}\right)$ :

$$
\Phi_{T}^{m}(T)=\frac{N}{S_{m}(T) / \rho}
$$

indicating that the equilibrium spectrum for an initially monoenergetic source of charged particles is directly proportional to the number of particles released per unit mass and is inversely proportional to the mass stopping power in the medium in which the particles slow down and stop. Using this electron spectrum, it is possible to express the dose to the cavity by: 


$$
D_{\mathrm{c}}=\int_{o}^{T_{o}} \frac{N}{S_{m}(T) / \rho} S_{\mathrm{c}}(T) / \rho d T
$$

resulting in the following expression for $D_{c} / D_{m}$ :

$$
\frac{D_{c}}{D_{m}}=\frac{1}{T_{0}} \int_{0}^{T_{0}} \frac{S_{\mathrm{c}}(T) / \rho}{S_{m}(T) / \rho} \rho d T .
$$

This equation is a more general form of equation Eq. (4-11) in the charged particles equilibrium condition, as long as the energy deposited by delta electrons is ignored.

To generalize, if energy escaping through bremsstrahlung photons is considered, the total energy deposited by the mono-energetic source is given by:

$$
D_{m}=N T_{0}\left(1-Y_{m}\left(T_{0}\right)\right)
$$

where $Y_{m}\left(T_{0}\right)$ is the radiation yield of an electron with energy $T_{0}$. The dose to the medium is then given by:

$$
D_{\mathrm{m}}=\int_{0}^{T_{0}} \Phi_{\mathrm{T}}^{\mathrm{m}}\left(S_{m}(T) / \rho\right)_{c o l l} d T
$$

whereas the dose to the cavity is given by:

$$
D_{\mathrm{c}}=\int_{0}^{T_{o}} \Phi_{\mathrm{T}}^{\mathrm{m}}\left(S_{\mathrm{c}}(T) / \rho\right)_{c o l l} d T
$$

The equilibrium fluence relation is as explained before:

$$
\Phi_{\mathrm{T}}^{\mathrm{m}}=\frac{N}{S_{m}(T) / \rho}
$$

and the ratio of the doses $D_{c} / D_{m}$ is then given by: 


$$
\frac{D_{\mathrm{c}}}{D_{\mathrm{m}}}=\frac{1}{T_{0}\left(1-Y_{m}\left(T_{0}\right)\right)} \int_{o}^{T_{o}} \frac{\left(S_{\mathrm{c}}(T) / \rho\right)_{\text {coll }}}{\left(S_{m}(T) / \rho\right)} \rho d T .
$$

\section{II.D. Electron spectra including secondary electrons}

When considering the production of secondary electrons in the slowing down of the primary electrons along their tracks, a similar approach as above can be followed, except that the conservation of energy equation now incorporates the cross section for hard collisions (Møller cross section). This approach was followed by Spencer ${ }^{6}$ in 1955 and following the same procedure, the equilibrium fluence is given by:

$$
\underset{T}{\Phi^{m, \delta}}=\frac{N R\left(T, T_{0}\right)}{S_{m}(T) / \rho}
$$

where $R\left(T, T_{0}\right)$ is the ratio of the electron fluence that includes $\delta$ rays to the primary electron fluence. Monte Carlo techniques are used to calculate electron equilibrium spectra by simulating the electron tracks according to the probability distribution functions obtained from the total and differential cross sections. Monte Carlo calculations often use a condensed history transport model, i.e., they assume that the electrons are transported in steps instead of simulating each interaction which is too intensive to perform. The size of the steps is determined from the probability of an elastic event and the probability of a hard collision. Since hard collisions imply a large transfer of the electron energy, between hard collisions the energy is lost by CSDA. The electron energy in the process of slowing down is distributed in discrete energy bins to form a spectrum.

The calculation of electron slowing down spectra from a mono energetic primary electron beam can be extended to calculate the spectrum of electrons resulting from an initial photon beam. For each primary electron that results from a photon interaction an electron spectrum is calculated. The contributions of all the primary electrons are scored to obtain the final spectrum. 


\section{II.E. Spencer-Attix cavity theory}

Spencer and Attix ${ }^{6}$ expanded the Bragg-Gray theory by showing that the effects of secondary electrons ( $\delta$ rays) must be taken into account in cavity theory. As incident electrons are slowed down in the medium, several low energy secondary electrons are produced resulting in an enhancement of the low energy portion of the spectra, as discussed in the previous section. The same basic assumptions of the Bragg-Gray theory also apply to the Spencer-Attix cavity theory; the two Bragg-Gray conditions must be met and the energy given to bremsstrahlung photons is negligible. Moreover, the Spencer-Attix cavity theory also requires that the cavity does not perturb the total electron fluence (including $\delta$ rays), whereas the Bragg-Gray approach only assumes this for the primary electron spectrum.

Spencer-Attix cavity theory allows the generation of $\delta$ electrons in the electron slowing down process, and the final spectrum takes into account all their contributions. The theory defines a threshold energy $\Delta$ which separates the electrons into two groups:

1. Slow electrons: their energy is lower than $\Delta$ and is deposited locally. It is impossible for them to cross the cavity and they do not contribute to the cavity dose.

2. Fast electrons: their energy is greater than $\Delta$ and they have enough energy to cross the cavity and deposit some of their energy within that cavity.

The choice of $\Delta$ is closely related to the size of the cavity, since it is defined as the energy of the electron with range equal to the dimension of the cavity. The calculation of the dose with the Spencer-Attix cavity theory will take into account the fluence spectrum which includes $\delta$ electrons with energies larger than $\Delta$ and less than $T_{\delta} / 2$, where $T_{0} / 2$ is the maximum energy transfer possible in a single electron-electron interaction. All electrons of the spectrum, including secondaries, are allowed to slow down and deposit their energy in the medium following the stopping power. However, the energy losses must be restricted to a minimum threshold value $\Delta$ and therefore the restricted stopping power, $L_{\Delta}(T) / \rho$, has to be used. The dose ratio medium to cavity is given by:

$$
\frac{D_{m}}{D_{c}}=\frac{\int_{\Delta}^{T_{0}} \Phi_{T}^{m, \delta} L_{\Delta, m}(T) / \rho d T+\mathrm{TE}_{m}}{\int_{\Delta}^{T_{0}} \Phi_{T}^{m, \delta} L_{\Delta, c}(T) / \rho d T+\mathrm{TE}_{c}}=\left(\frac{\bar{L}_{\Delta}}{\rho}\right)_{c}^{m}
$$


where $T E_{m}$ and $T E_{c}$ are the track-end terms. Track-end terms account for the energy deposited at the end of tracks by electrons that have an initial energy between $\Delta$ and $2 \Delta$ which can have an energy drop below $\Delta$ and therefore have to deposit their total energy locally. Track-end terms were approximated by $\mathrm{Nahum}^{7}$ as:

$$
\mathrm{TE}_{m}=\Phi_{T}^{m, \delta}(\Delta) \frac{\mathrm{S}_{\mathrm{m}}(\Delta)}{\rho} \Delta
$$

and

$$
\mathrm{TE}_{\mathrm{c}}=\Phi_{T}^{m, \delta}(\Delta) \frac{\mathrm{S}_{\mathrm{c}}(\Delta)}{\rho} \Delta
$$

These equations indicate that the portion of the track where the energy of the electron is below $\Delta$ deposits an amount of energy per unit mass equal to the product $\Delta$, and the total electron spectrum with the unrestricted stopping powers both evaluated at the threshold $\Delta$. Unrestricted stopping power has to be used for the TE terms, since an electron with energy $\Delta$ can transfer a maximum energy $\Delta / 2$. The track-end energy deposition contributes from $5 \%$ to $10 \%$ of the total dose.

\section{II.F. Cavity theory for photon beams}

Bragg-Gray and Spencer-Attix cavity theories both require that no electrons are created by photon interactions within the cavity. However, the production of electrons in the cavity can be significant when irradiating with a photon beam, especially for large cavities and lower photon beam energies. Under charged particle equilibrium conditions, for very large cavities or at very low energies, the dose ratio medium to cavity becomes a function that depends purely on the ratio of the mass energy absorption coefficients:

$$
\frac{D_{m}}{D_{c}}=\left(\frac{\bar{\mu}_{a b}}{\rho}\right)_{c}^{m} .
$$


For intermediate conditions, Burlin ${ }^{8}$ developed a weighted method in which the ratio of dose to medium to dose to cavity is given by:

$$
\frac{D_{c}}{D_{m}}=d\left(\frac{\bar{L}_{\Delta}}{\rho}\right)_{m}^{c}+(1-d)\left(\frac{\bar{\mu}_{a b}}{\rho}\right)_{m}^{c}
$$

where $d$ is a parameter that corresponds to the average value of the electron fluence reduction in the medium, and is defined as:

$$
d=\frac{\int_{0}^{L} \Phi_{m}^{e} e^{-\beta l} d l}{\int_{0}^{L} \Phi_{m}^{e} d l}
$$

with $l$ the distance of any point in the cavity to the wall, $L$ the mean cavity size, and $\beta$ the effective attenuation coefficient which represents the reduction in particle fluence from the medium through $(1-d)$, and the increase in fluence through interactions within the cavity. This two-component model is also used for chamber wall correction factors that could be applied to Spencer-Attix cavity theory.

\section{Calibration PROtocols}

The goal of clinical dosimetry calibration protocols is to determine the absorbed dose to water delivered by a clinical radiation beam under a specific set of reference conditions in order to deliver accurately a prescribed dose to a patient's planning target volume. The International Commission on Radiation Units and Measurements (ICRU) ${ }^{9}$ recommends an overall accuracy in the dose delivery of $\pm 5 \%$ based on an analysis of dose response data and an evaluation of errors in dose delivery in a clinical setting.

The calibration of an external radiation beam is the dose rate given in cGy/min for $\mathrm{x}$-ray and isotope teletherapy machines and in $\mathrm{cGy} /$ monitor unit (cGy/MU) for linacs at depth of dose maximum in a water phantom for a nominal source to surface distance (SSD) and a nominal field size of $10 \times 10 \mathrm{~cm}^{2}$ on the phantom surface, as illustrated in Fig. 4-6. 
The calibration of clinical photon and electron beams is normally carried out with ionization chambers which have calibration factors determined either in air or in water, traceable to a national primary standards dosimetry laboratory. A chamber can be directly calibrated at a national laboratory or can be cross-calibrated with another ionization chamber (a local secondary standard calibrated at a national standards laboratory).

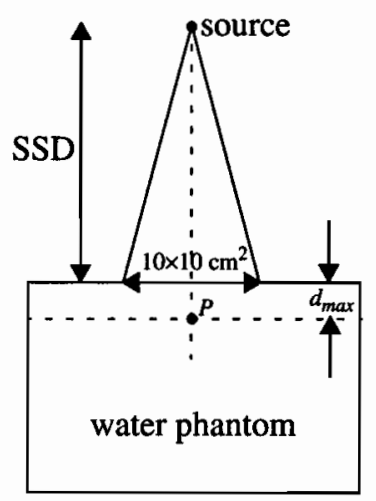

Figure 4-6. Basic beam output calibration setup. The point of calibration $P$ is at depth $d_{\max }$ in a water phantom that is irradiated by a $10 \times 10 \mathrm{~cm}^{2}$ field produced by a source located at a distance SSD from the surface of the phantom.

Historically there are three main protocol types; listed chronologically:

1. procedures based on exposure measurements in a phantom $\left(C_{\lambda}\right)$ (before 1980)(Ref. \#9).

2. procedures based on exposure in air or air-kerma in air calibrations and Spencer-Attix cavity theory (between 1980 and 2000) (AAPM TG-21 (Ref. \#10), AAPM TG-25

(Ref. \#11) and IAEA TRS-277 (Ref. \#12-14)).

3. procedures based on absorbed dose calibrations (after 1999) (IAEA TRS-398 (Ref. \#15), AAPM TG-51 (Ref. \#16)).

The first approach is now only used for kilovoltage $x$-ray dosimetry, because of conceptual difficulties. The two latter protocol-types make use of Spencer-Attix cavity theory to determine the absorbed dose to water and the dose determination procedure depends on the chamber dimensions, radiation type, and energy used. They cover one or more of several distinct beam types and energy ranges, such as: (i) low energy (superficial) 
$\mathrm{x}$-ray beams; (ii) medium energy x-ray beams (orthovoltage); (iii) megavoltage $\mathrm{x}$-ray beams; (iv) electron beams below $10 \mathrm{MeV}$; and ( $v$ ) electron beams equal or above $10 \mathrm{MeV}$.

\section{III.A. Equipment}

The most commonly used equipment to calibrate a clinical radiation beam is an ionization chamber system. This system consists of a suitable ionization chamber, an electrometer, and a power supply connected in an electrical circuit in which the chamber acts as a capacitor and the electrometer measures the charge created by radiation and collected in the sensitive volume of the chamber.

As illustrated in Fig. 4-7, the sensitive air volume of an ionization chamber is defined by three electrodes: (i) the polarizing electrode connected directly to the power supply; (ii) the measuring electrode which is connected to ground through a low impedance electrometer; (iii) the guard electrode which is directly grounded firstly to define the sensitive volume and secondly to prevent measurement of leakage currents.

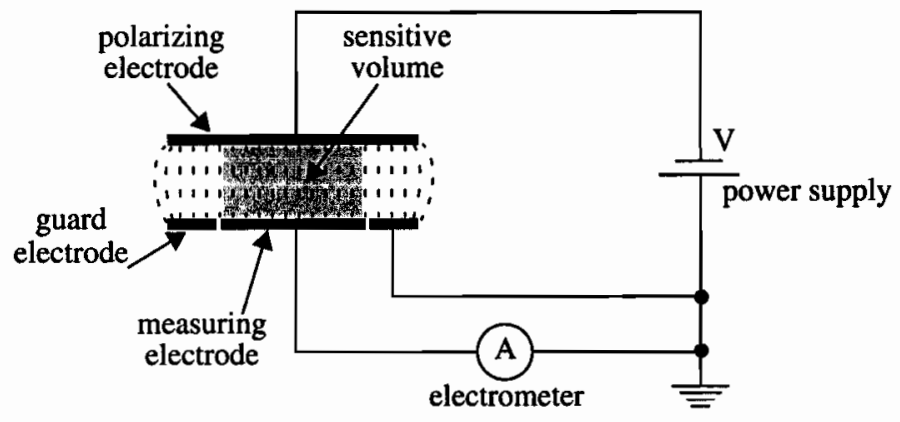

Figure 4-7. Schematic diagram of an ionization chamber circuitry for a typical radiation dosimetry system.

From a geometrical point-of-view there are two types of ionization chambers used for radiation dosimetry calibration: cylindrical and parallel-plate chambers. Cylindrical chambers are the most commonly used chambers for orthovoltage and megavoltage $\mathrm{x}$-ray beams and electron beams of $9 \mathrm{MeV}$ and above, while parallel-plate chambers are used for superficial $x$ ray and low energy electron beams. 
The charge that is produced by radiation in the sensitive volume of the chamber is very small and must be measured by a very sensitive charge or current measuring device (electrometer) with a high input impedance $\left(\geq 10^{14} \Omega\right)$. The power supply is either a stand alone unit or is part of the electrometer device, and must provide constant potential but variable in magnitude and in polarity.

Protocols ultimately yield the value of absorbed dose in a water equivalent phantom. However, clinical dosimetric measurements are often carried out in more practical solid materials such as polystyrene, Lucite, and Solid Water ${ }^{\mathrm{TM}}$. For photon dosimetry an equivalent water material should have the same energy absorption coefficient, and mass stopping power as water. For electron dosimetry, the phantom material should have the same linear stopping power and linear scattering power as water. However, the use of water is generally recommended as phantom material for calibration of photon and electron beams. Standard calibration is performed at a depth of $10 \mathrm{~cm}$ in phantom for photon beams, and for electron beams, at a depth $d_{r e f}$, defined as $d_{r e f}=0.6 R_{50}-0.1(\mathrm{~cm})$, where $R_{50}$ is the depth in water at which the percent depth dose of a $10 \times 10 \mathrm{~cm}^{2}$ field falls at $50 \%$ of the dose maximum, as shown in Fig. 1-3 (B). For electron beams with energy greater than 20 $\mathrm{MeV}$, a $20 \times 20 \mathrm{~cm}^{2}$ field is required. The phantom should be at least $5 \mathrm{~cm}$ larger than the $10 \times 10 \mathrm{~cm}^{2}$ field in all directions.

\section{III.B. Beam quality specification}

The absorbed dose at the reference point is calculated from the measured signal produced by radiation in the ionization chamber at that point. The raw charge or current signal has to be multiplied by various dosimetric physical quantities to yield the actual machine output. The physical quantities depend not only on the chamber geometry and properties but also on radiation beam type and energy. A precise method for specifying the beam quality $Q$ of a radiation beam has to be followed. The $Q$ value of a radiation beam is be used to select the proper correction factors that are provided in dosimetry protocols. 


\section{III.B.1 Photon beams}

For low and medium energy $\mathrm{x}$ rays, called superficial and orthovoltage beams, respectively, the beam quality specifiers are the tube potential, the total filtration, and a measurement of the first half-value layer under narrow beam geometry conditions. For high energy $x$ rays (megavoltage) either the tissue-phantom ratio (TPR) or the percentage depth dose at depth of $10 \mathrm{~cm}$ in water for a $10 \times 10 \mathrm{~cm}^{2}$ field are used for the beam quality specification. The choice depends on the particular radiation dosimetry protocol used.

\section{III.B.2 Electron beams}

The electrons generated by a linear accelerator are essentially monoenergetic as they exit the waveguide. The electrons, while travelling through the various components of the treatment machine head, such as the scattering foil, the collimators, the ion chamber and the electron applicator as well as air, will undergo scattering and lose energy. Thus, the beam reaching the patient surface is composed of a spectrum of electron energies. The mean energy at the phantom surface $\bar{E}(0)$ and the most probable energy on the phantom surface $E_{p}(0)$ are approximated by empirical formulae ${ }^{17}$ as:

$$
\bar{E}(0)=C_{0} R_{50},
$$

and

$$
E_{p}(0)=C_{1}+C_{2} R_{p}+C_{3} R_{p}^{2}
$$

where $C_{0}=2.33 \mathrm{MeV} / \mathrm{cm}, C_{1}=0.22 \mathrm{MeV}, C_{2}=1.98 \mathrm{MeV} / \mathrm{cm}$, and $C_{3}=0.0025 \mathrm{MeV} / \mathrm{cm}^{2}$, and $R_{50}$ and $R_{p}$, are, respectively, the depth of the $50 \%$ dose point and the practical range of the electron, as it was illustrated in Fig. 1-3 (B), both measured in water phantom. If a plastic phantom is used for the measurements, the depths have to be corrected with a material scaling factor $\left(C_{p l}\right)$ such that:

$$
R_{\text {water }}=R_{\text {plastic }} C_{p l}
$$


IAEA (TRS-381) protocol defines $C_{p l}$ as the ratio of the average electron penetration in water and plastic and equivalently AAPM TG-25 defines it as the effective density.

The mean electron beam energy at depth in phantom $\bar{E}(z)$ is approximated by the Harder relationship as:

$$
\bar{E}(z)=\bar{E}(0)\left[1-\frac{z}{R_{p}}\right]
$$

where $\bar{E}(0)$ and $R_{p}$ were defined above. Harder's relation is used for electron beams with energies less than $10 \mathrm{MeV}$ or for small depths at higher beam energies. Tabulated data of $\bar{E}(z) / \bar{E}(0)$ versus the scaled depth $z / R_{p}$ are used to obtain $\bar{E}(z)$ when Harder's relation is not applicable ${ }^{12}$. All recent electron dosimetry protocols use $R_{50}$ for the determination of the beam quality conversion factor.

\section{III.C. Basic calibration factor}

As already noted, clinical dosimetry protocols are based on either in-air or in-water calibration factors. The calibration of radiotherapy beams, including cobalt- 60 gamma-ray beams, megavoltage x-ray beams and electron beams, is based on a chamber calibration obtained in a cobalt- 60 gamma-ray beam traceable to a national standards laboratory.

In-air calibration factors are based on either exposure measurements in air to provide exposure calibration factors $N_{X}$ or on air-kerma in air measurements to provide air-kerma calibration factors $N_{K}$. The two factors are related through the following relationship:

$$
N_{K}=N_{X} \bar{W}_{a i r} \frac{1}{1-g}
$$

where $\bar{W}_{\text {air }}$ is the mean energy required to produce an ion pair in air $(33.97 \mathrm{eV})$, and $g$ is the bremsstrahlung fraction ( 0.3 for cobalt radiation), as defined in section Section IV.C of Chapter 1 and Section IV.J of Chapter 2, respectively. 
The calibration factor in water $N_{D}$ is usually measured in a cobalt-60 beam and is based on a measurement response to dose in water. The absorbed dose to air chamber factor $N_{D \text {,air }}$, denoted $N_{g a s}$ in AAPM TG-21, is determined from $N_{K}$ from the following relationship:

$$
N_{D, \text { air }}=N_{K}(1-g) k_{a t t} k_{m}
$$

where $k_{m}$ is a factor correcting for the lack of air equivalence of the ionization chamber material at the calibration in the cobalt- 60 gamma-ray beam and $k_{a t t}$ is a correction factor taking into account the attenuation and scatter of photons in the ionization chamber walls and build up cap, and $g$ is again the bremsstrahlung fraction.

$N_{D, a i r}$ is a unique characteristic of each ionization chamber, since it is closely related to the sensitive air volume $V$ of the particular chamber. When a charge $Q$ is produced in the air mass $m_{a i r, c}$ of the chamber cavity, the absorbed dose to air in the cavity is given by:

$$
D_{a i r, c}=\frac{Q}{m_{a i r, c}} \bar{W}_{a i r}=\frac{Q}{\rho_{a i r, c} V} \bar{W}_{a i r}
$$

where $\rho_{a i r, c}$ is the density of air in the cavity. The air volume $V$ is generally not known to the required accuracy for calibration, and for that reason, it is indirectly determined through the chamber calibration in a standard cobalt-60 beam. The ratio $D_{a i r, c} d Q$ is assumed to be constant at cobalt-60 energy and $N_{D \text {,air }}$ is then defined as:

$$
N_{D, a i r}=\frac{1}{\rho_{\text {air }} V} \bar{W}_{\text {air }}
$$

Since $\bar{W}_{\text {air }}$ is generally assumed to be constant for all beam energies used in radiotherapy, $N_{D, \text { air }}$ is also constant for all beam energies and is an indirect measure of the chamber cavity volume. Thus, $N_{D \text {, air }}$ can be used for calibrating the chamber at beam energies other than cobalt- 60 .

Many measurement parameters influence the reading of the chamber. They have to be accounted for through the use of various correction factors in order to apply the basic calibration factor $N_{i}$ and finally obtain an absolute measure of the absorbed dose. 


\section{III.D. Chamber reading correction factors}

\section{III.D.1 Temperature and pressure correction}

Temperature and pressure variations affect the mass of air inside an ionization chamber. Since the total charge produced in the cavity of an ionization chamber depends on the mass of air in the cavity, the collected charge depends on the air temperature and pressure. The basic calibration factor of the chamber is obtained at the reference temperature $T_{0}$ and pressure $P_{0}$, with $P_{0}=101.3 \mathrm{kPa}$ and $T_{0}=20^{\circ} \mathrm{C}$ in Europe and $T_{0}=22^{\circ} \mathrm{C}$ in North America. The correction factor $C_{T, P}$ normalizes the chamber readings to the same reference conditions by scaling the reciprocal of the air density $1 / \rho_{\text {air }}$ of Eq. (4-40) by:

$$
C_{T, P}=\frac{P_{0}}{P}\left[\frac{273.15+T}{273.15+T_{0}}\right]
$$

where $T$ and $P$ are the ambient temperature in degrees Celsius and pressure in kilopascals during the beam output calibration.

\section{III.D.2 Polarity effect}

During irradiation, high energy electrons can cause various effects on the collecting electrode, the stem, or the cable of the ionization chamber. These effects yield spurious ionization signals affecting the measured signal. Low energy electrons that are deposited directly onto the collecting electrode or in the central wire of the cables will contribute to the ionization current by adding to, or subtracting from the measured current, depending on the polarity of the chamber at the time of measurement. Moreover, high energy electrons may cause the emission of secondary electrons from any of the components of the chamber that will also alter the charge reading. To account for these effects the chamber signal should be measured for both polarities of the biasing voltage. If a polarity effect is observed, the mean of the absolute values of the readings should be used as the measured signal, such that: 


$$
M_{c o r r}=\frac{\left|M_{+}\right|+\left|M_{-}\right|}{2}
$$

where $\left|M_{+}\right|$and $\left|M_{-}\right|$are the absolute values of the electrometer readings for positive and negative polarities, respectively. This method corrects well for all spurious currents that do not change the sign when the polarity is changed; for example, the irradiation of the stem and cable of the ionization chamber. However, other effects which change sign with a change in polarity cannot be accounted for with the simple signal averaging technique. An extensive review of polarity effects was given by Boag ${ }^{18}$. A change of polarity on the chamber requires that the conditions of charge equilibrium should be reestablished by conditioning the chamber through pre-irradiation and waiting several minutes before the next measurement.

\section{III.D.3 Ionic recombination correction factor $\mathbf{P}_{\text {ion }}$ •}

This factor accounts for the loss of ions in the chamber sensitive volume due to initial and general recombination as well as ionic diffusion against the electric field ${ }^{18}$. A two-voltage technique is generally used to determine the correction factor $P_{\text {ion }}$ by measuring chamber signal at two different voltages, $V_{H}$ and $V_{L} . P_{i o n}$ at the normal operating voltage $V_{H}$ is then given by: ${ }^{14}$

$$
P_{i o n}=a_{0}+a_{1}\left(\frac{\bar{M}_{H}}{\bar{M}_{L}}\right)+a_{2}\left(\frac{\bar{M}_{H}}{\bar{M}_{L}}\right)^{2}
$$

where $a_{i}$ are tabulated constant values for pulsed and pulsed-scanned radiation beams, and $\bar{M}_{H}$ and $\bar{M}_{\mathrm{L}}$ are the mean of the absolute values of the collected chamber signals at bias voltages $V_{H}$ and $V_{L}$, respectively. The ratio $V_{H} / V_{L}$ should be equal or larger than $3^{19,20}$ and $V_{H}$ should not be too high in order to avoid an over estimate the measured signal ${ }^{21,22}$ through charge multiplication effects in the sensitive volume of the chamber or radiation induced conduction effects. Details on ionic recombination are given in Chapter 7. After a change of bias voltage, it is important to reestablish the charge equilibrium in the chamber circuit, and a pre-irradiation of the chamber is recommended before the next measurement. 
It is recommended not to use ionization chambers that have a large ion recombination correction factor $(>2 \%)$. This correction depends on the dose to the air in the ion chamber per pulse of radiation. Therefore, the effect varies with dose rate and pulse rate. The variation is likely to affect measurement of depth doses, if an instrument with a large value of $P_{\text {ion }}$ is used.

\section{III.D.4 Air humidity correction}

A humidity correction factor is generally not needed because the basic calibration factor is referred to a relative humidity of $50 \%$ and the chamber is used in a relative humidity between $20 \%$ to $80 \%$ range for which the effect of humidity change is negligible, as discussed in section Section IV.C of Chapter 1.

\section{III.D.5 Leakage}

The leakage current is the signal measured by the electrometer when bias voltage is applied to the chamber but there is no irradiation. This current should always be evaluated and compared to the signal obtained during a given irradiation, and it should always be less than $0.1 \%$ of the signal read during irradiation. When the leakage current exceeds $0.1 \%$ of the signal measured with beam-on, a correction should be made to account for the extra signal.

\section{III.E. Output Calibration}

\section{III.E.1 Output calibration of low energy (superficial) x-ray beams (10 kVp-100 kVp)}

For superficial x-ray beams, the dose rate is determined at the surface $(d=0)$ of a water equivalent phantom. Protocols require the use of parallel plate ionization chambers that have thin entrance windows to simplify the positioning at the phantom surface. The appropriate air-kerma in air calibration factor $N_{K}$ of the chamber must be known, or the exposure calibration factor $N_{x}$, if known, could be used to calculate $N_{K}$, with 
$N_{K}=N_{X} \bar{W}_{a i r}$. Finally, the absorbed dose to water at the surface of the phantom, in the absence of the chamber, is given by:

$$
D_{w a t}(0)=M N_{X} B\left(\frac{\mu_{a b}}{\rho}\right)_{a i r}^{w a t}
$$

where $M$ is the measured chamber current or charge corrected for temperature, pressure and ionic recombination, $\mathrm{B}$ is the back-scatter factor, and $\left(\bar{\mu}_{a b} / \rho\right)_{a i r}^{\text {wat }}$ is the ratio of averaged mass energy absorption coefficients, water to air.

\section{III.E.2 Output calibration of medium energy (orthovoltage) x-ray beams (100 kVp-300 kVp)}

The absorbed dose can be determined at a given depth $d$ in a water phantom (usually $d=2 \mathrm{~cm}$ ) with a calibrated cylindrical ionization chamber by using the air-kerma calibration factor $N_{K}$ of the chamber in the following relationship:

$$
D_{w a t}(d)=M N_{K}\left(\frac{\bar{\mu}_{a b}}{\rho}\right)_{a i r}^{w a t} p_{u}
$$

where $p_{u}$ is the chamber perturbation correction factor and $\left(\mu_{a b} / \rho\right)_{a i r}^{\text {wat }}$ is the ratio of averaged mass energy absorption coefficients, water to air.

\section{III.E.3 Output calibration of megavoltage photon beams based upon air-kerma calibration factor}

Cylindrical ionization chambers are used to calibrate megavoltage photon beams at a given depth $d$ of $5 \mathrm{~cm}$ or $10 \mathrm{~cm}$ in a water phantom. The air-kerma in air calibration factor $N_{K}$ obtained in a cobalt-60 beam at a standards laboratory is used. Tissue-phantom ratio at depth of $20 \mathrm{~cm}$ and $10 \mathrm{~cm}\left(T P R_{20,10}\right)($ Ref. \#14) or the percentage depth dose at depth $10 \mathrm{~cm}(P D D(10))($ Ref. \#15) yields the beam quality. A cavity theory (Bragg-Gray or Spencer-Attix) is used to determine the absorbed dose rate at the point of measurement at depth $d$ in the phantom. The following relationship is used: 


$$
D_{\text {wat }}(d)=M N_{D, a i r}\left(\frac{\bar{L}}{\rho}\right)_{a i r}^{\text {wat }} p_{\text {wall }}
$$

where $M$ is the corrected (for temperature, pressure, polarity and recombination) measured chamber current or charge, $(\bar{L} / \rho)_{\text {air }}^{\text {wat }}$ is the restricted stopping power ratio between water and air chosen according to the determined beam quality, and $p_{\text {wall }}$ is a perturbation factor, based on the Burlin theory of Eq. (4-31) that takes into account the wall chamber effects and the electron fluence perturbation due to the insertion of an air cavity into the water phantom. $p_{\text {wall }}$ is estimated from:

$$
p_{\text {wall }}=\frac{\alpha\left(\frac{S}{\rho}\right)_{\text {air }}^{\text {wall }}\left(\frac{\mu}{\rho}\right)_{\text {wall }}^{\text {wat }}+\tau\left(\frac{S}{\rho}\right)_{\text {air }}^{\text {sleeve }}\left(\frac{\mu}{\rho}\right)_{\text {sleeve }}^{\text {wat }}+(1-\alpha-\tau)\left(\frac{S}{\rho}\right)_{\text {air }}^{\text {med }}}{\left(\frac{S}{\rho}\right)_{\text {air }}^{\text {water }}}
$$

where $\alpha$ and $\tau$ are estimated from the thicknesses of the chamber wall $\left(t_{\text {wall }}\right)$ and the sleeve $\left(t_{\text {sleeve }}\right)$ in $\mathrm{g} / \mathrm{cm}^{2}$ in the following relationship:

$$
\begin{aligned}
& x=1-e^{-11.88 t_{\text {wall }}} \\
& \text { and } \tau=e^{-11.88 t_{\text {wall }}}-e^{-11.88\left(t_{\text {wall }}+t_{\text {sleeve }}\right)} .
\end{aligned}
$$

Since the chamber volume is cylindrical, the effective point of measurement has to be corrected. It is located in front of the chamber centre to correct for the gradient of the electron fluence within the chamber cavity.

\section{III.E.4 Output calibration of megavoltage electron beams based upon air-kerma calibration factor}

For electron beam with energies equal to or above $10 \mathrm{MeV}$ a cylindrical ionization chamber is used at depth of dose maximum in a water phantom. At energies below 10 $\mathrm{MeV}$, a plane-parallel plate chamber is used in a plastic phantom. The calibration is based on the air-kerma in air calibration factor $N_{K}$ obtained in a cobalt-60 beam at the standards laboratory. The beam quality is specified by the mean electron energy on the phantom sur- 
face $\bar{E}(0)$. Again, Bragg-Gray or Spencer-Attix cavity theory is used to determine the absorbed dose rate at the reference point in the phantom:

$$
D_{w a t}(d)=M N_{D, a i r}\left(\frac{\bar{L}}{\rho}\right){ }_{a i r}^{w a t} p_{c a v}
$$

where $M$ is the temperature, pressure, polarity and recombination corrected chamber reading, $(\bar{L} / \rho)_{\text {air }}^{\text {wat }}$ is the restricted stopping power ratio between water and air, and $p_{c a v}$ is the cavity perturbation factor given by: ${ }^{14}$

$$
p_{c a v}\left(\bar{E}_{0}, r\right)=1-0.02155 r e^{-0.1224 \bar{E}_{z}}
$$

where $r$ is the cavity radius in $\mathrm{mm}$ and $\bar{E}_{z}$ is the mean electron energy at depth $z$ defined in Eq. (4-36).

\section{III.E.5 Output calibration of megavoltage photon beams based upon dose to water calibration factor (AAPM TG-51 protocol)}

A cylindrical ionization chamber is used at the reference depth $d$ (normally $d=10 \mathrm{~cm}$ ) in a water phantom. The basic output calibration factor is based on a dose to water chamber calibration factor $N_{D, w}, C o$ obtained from a standards laboratory with the chamber irradiated in a cobalt-60 beam at a reference depth $d_{r e f}$ in a water phantom. The absorbed dose to water $D_{w, C o}$ at the reference depth in phantom in a cobalt-60 beam, in the absence of the ionization chamber, is given by:

$$
D_{w, Q}=M_{C o} N_{D, w, C o}
$$

where the $M_{C o}$ is the chamber signal corrected for ambient temperature, pressure, polarity, as well as recombination loss $\left(P_{\text {ion }}\right)$.

When the ionization chamber is used in a beam quality $Q$, which is different from the calibration cobalt- 60 beam quality, the absorbed dose is given by:

$$
D_{w, Q}=M_{Q} N_{D, w, C o} k_{Q, C o}
$$


where $k_{Q, C o}$ is a correction factor that takes into account the difference between the reference beam quality $C o$ and the user beam quality $Q$. The chamber signal $M_{Q}$ must be corrected to the reference values (temperature, pressure, ion recombination, polarity effect, and leakage)

The beam quality $Q$ of a megavoltage photon beam is specified either with a ratio of tissue-phantom ratios $^{14}\left(T P R_{20,10}(Q)\right)$ or with the percentage depth dose ${ }^{15}$ $P D D(10,10 \times 10, S S D, Q)$. The ratio of TPR is given by:

$$
T P R_{20,10}=\frac{T P R(20,10 \times 10, Q)}{\operatorname{TPR}(10,10 \times 10, Q)}
$$

and is measured at depths of $20 \mathrm{~cm}$ and $10 \mathrm{~cm}$ in a water phantom with a field size of $10 \times 10 \mathrm{~cm}^{2}$ at the source-axis distance $S A D$ (from the source to the point of measurement in phantom).

The $P D D(10,10 \times 10, S S D, Q)$, on the other hand, is measured at a depth of 10 $\mathrm{cm}$ in a water phantom at nominal source-surface distance $S S D$ and a field size of $10 \times 10 \mathrm{~cm}^{2}$.

IAEA dosimetry protocols ${ }^{15,23}$ recommend the use of the ratio of TPRs, while the AAPM TG-51 (Ref. \#16) protocol recommends the use of the PDD for beam quality specification. In practice, the two approaches yield essentially the same results.

The beam quality correction factor $k_{Q, C_{o}}$ is defined as a ratio, at beam quality $Q$ and $\mathrm{Co}$, of the calibration factors in terms of the absorbed dose to water of the ionization chamber as:

$$
k_{Q, C o}=\frac{N_{D, w, Q}}{N_{D, w, C o}}=\frac{D_{w, Q} / M_{Q}}{D_{w, C o} / M_{C o}} .
$$

The beam quality correction factors should be measured directly for each ionization chamber at the same beam quality of the local clinical beams. In practice this is not possible and the factors are calculated from the Bragg-Gray theory as: 


$$
k_{Q, C o}=\frac{\left[\left(\frac{S}{\rho}\right)_{a i r}^{w a t}\right]_{Q}\left[\bar{W}_{a i r}\right]_{Q} p_{Q}}{\left[\left(\frac{S}{\rho}\right)_{\text {air }}^{\text {wat }}\right]_{C o}\left[\bar{W}_{a i r}\right]_{C o} p_{C o}}
$$

Assuming that $\left[\bar{W}_{a i r}\right]_{Q}=\left[\bar{W}_{a i r}\right]_{C o}$, we can write $k_{Q, C o}$ as follows:

$$
k_{Q, C o}=\frac{\left[\left(\frac{S}{\rho}\right)_{\text {air }}^{\text {wat }}\right.}{]_{Q} p_{Q}}
$$

showing that $k_{Q, C o}$ depends only on quotients of water to air stopping power ratios and perturbation factors at beam qualities $Q$ and $C o$. The relationship between $N_{K}$ and $N_{D \text {,air }}$ of the IAEA TRS- $277^{12}$ and TRS- $381^{23}$ protocols, and the new $N_{D, w, C o}$ formalism of IAEA TRS- $398^{15}$ is given, for megavoltage photon beams, by:

$$
N_{D, w, C o}=N_{D, \text { air }}\left[\left(\frac{S}{\rho}\right)_{\text {air }}^{\text {wat }}\right] p_{C o}
$$

where $p_{C o}$ is the overall perturbation factor given by:

$$
p_{C o}=\left[p_{d i s} p_{\text {wall }} p_{c a v} p_{c e l}\right]_{C o}
$$

where $p_{\text {dis }}$ is a factor that accounts for the effect of replacing a volume of water with the chamber cavity; $p_{\text {wall }}$ is a factor that corrects for the response of the ionization chamber for the non-water equivalence of the chamber wall and any waterproofing material; $p_{c a v}$ is a factor that corrects for the response of the ionization chamber for effects related to the air cavity, predominantly the in-scattering of electrons that makes the electron fluence inside the cavity different from that in water in the absence of the cavity; and finally $p_{c e l}$ is a factor that corrects for the response of the chamber for the effect of the central electrode during in-phantom measurements. 


\section{III.E.6 Output calibration of megavoltage electron beams based upon dose to water calibration factor (AAPM TG-51 protocol)}

The output calibration factor is based upon a dose to water chamber calibration factor $N_{D, w, C o}$ obtained from a standards laboratory with the chamber irradiated in a reference beam of quality $Q_{o}$. Generally that quality factor is for a cobalt-60 gamma-ray beam but dosimetry protocols also allow its use for calibration of electron beams. Again, a distinction is made for electron beams of energies below and above and equal to $10 \mathrm{MeV}$. For electron beam energies below $10 \mathrm{MeV}$ a plane-parallel plate chamber must be used and is also recommended for energies higher than $10 \mathrm{MeV}$. The reference point of measurement is taken to be on the inner surface of the entrance window, at the center of the electrode. The use of a water phantom is recommended, and the phantom should extend to at least 5 $\mathrm{cm}$ beyond all four sides of the largest field size. The use of a plastic phantom is allowed for energies below $10 \mathrm{MeV}$, but measuring depths must be scaled accordingly.

The beam quality specifier for electron beams is $R_{50}$, the depth at which $P D D$ is half the maximum value. When measuring $R_{50}$, the field size must be at least $10 \times 10 \mathrm{~cm}^{2}$ for $R_{50}<7 \mathrm{~g} / \mathrm{cm}^{2}$ and at least $20 \times 20 \mathrm{~cm}^{2}$ for $R_{50}>7 \mathrm{~g} / \mathrm{cm}^{2} . R_{50}$ is measured with a plane-parallel chamber in a water phantom.

The output calibration must be carried out in a water phantom at a reference depth $z_{\text {ref }}$ given by:

$$
\dot{z}_{r e f}=\left(0.6 R_{50}-0.1\right) \mathrm{g} / \mathrm{cm}^{2}
$$

with $R_{50}$ in $\mathrm{g} / \mathrm{cm}^{2}$. The reference depth $z_{r e f}$ is close to the depth of dose maximum $z_{\max }$ for beam qualities $R_{50}<4 \mathrm{~g} / \mathrm{cm}^{2}\left(E_{0}<10 \mathrm{MeV}\right)$ and is deeper than $z_{\max }$ for higher electron beam energies.

Finally, the absorbed dose to water at the reference depth $z_{\text {ref }}$ in an electron beam of quality $Q$, in the absence of the chamber is given by;

$$
D_{w, Q}=M_{Q} N_{D, w, C o} k_{Q, C o}
$$


where $M_{Q}$ is again the corrected chamber signal, and $k_{Q, C o}$ is the chamber correction factor that corrects the differences between the reference beam quality of cobalt-60 $(\mathrm{Co})$ and the measured electron beam quality $Q$.

The calculated values of $k_{Q, C o}$ are available as a function of $R_{50}$ in the dosimetry protocol documents for a wide variety or clinically used plane-parallel and cylindrical ionization chambers.

\section{SUMmARY}

Ionizing radiation beams transfer directly or indirectly some of their energy to the medium in which they travel. Some of that energy is eventually absorbed by the medium and accurate measurement of absorbed dose is the main goal of radiation dosimetry. For indirectly ionizing particles, such as photons, kerma is the quantity that relates most explicitly the description of the radiation and its effects. Kerma can also be directly related to the absorbed dose under charge particle equilibrium conditions.

Cavity theories were developed for relating the absorbed dose to a small cavity to the dose to the medium in the presence of the cavity. Bragg-Gray cavity theory requires that the cavity dimensions be small relative to the range of the charged particles generated in the vicinity of the cavity and that no charged particles are generated or stopped within the cavity. The main Bragg-Gray theory limitation is that it does not take into account secondary electrons, $\delta$ rays, created in the medium. Spencer-Attix cavity theory has the same basic assumptions and requirements as the Bragg-Gray cavity theory but also includes the effects of $\delta$-ray electrons by incorporating them into the equilibrium charged particle spectrum. Spencer-Attix defines a threshold energy value $\Delta$ that categorizes electrons into two different groups, slow electrons and fast electrons, in relation to their ability to travel across the cavity (fast) or not (slow).

The goal of calibration protocols is to determine accurately the absorbed dose to water delivered by a clinical radiation beam under a specific set of reference conditions in order to deliver accurately a prescribed dose to a patient's tumour volume. Various recognized protocols provide a method of calibrating a radiation beams with an ionization cham- 
ber using a basic output calibration factor obtained from a standards laboratory. The basic output calibration factor is generally obtained in a cobalt- 60 reference beam. The value of the basic calibration factor $N$ is directly related to the sensitive volume of a particular chamber which is difficult to determine accurately by other means. The concept of the procedure is either based upon an air-kerma calibration factor (AAPM TG-21, AAPM TG-25, IAEA TRS-277) or upon an absorbed dose to water calibration factor (AAPM TG-51, IAEA TRS-398).

Numerous correction factors have to be applied in dosimetry protocols when calculating the dose to account for polarity effects, reference temperature and pressure, ion recombination, and finally fluence correction for the presence of the chamber wall, cavity, and electrodes. 


\section{References}

1 ICRU, "Radiation quantities and units," ICRU Report 33, International Commission on Radiation Units and Measurements, Washington, D.C. (1980).

2 Harold Elford Johns and John Robert Cunningham, The physics of radiology, 4th ed. (Charles C. Thomas, Springfield, Ill., U.S.A., 1983).

3 D. W. O. Rogers, "Ionizing Radiation Dosimetry and Medical Physics," Physics in Canada 51, 178-181 (1995).

4 W. H. Bragg, "Consequences of the corpuscular hypothesis of the gamma and $\mathrm{x}$ rays, and the ranges of beta rays," Phil. Mag. 20, 385 (1910).

5 L.H. Gray, “Absorption of penetrating radiation," Proc. Roy. Soc. A. (London) 122, 647 (1929).

6 L. V. Spencer and F. H. Attix, "A theory of cavity ionization," Radiat. Res. 3, 239-254 (1955).

7 A. E. Nahum, "Extension of the Spencer-Attix Cavity Theory to the 3 media situation for Electron beams," in Dosimetry in Radiotherapy, edited by IAEA (IAEA, Vienna, 1988), Vol. 1, pp. 87-115.

8 T. E. Burlin, "The measurement of exposure dose for high energy radiation with cavity ionization chambers," Phys. Med. Biol. 3, 197-206 (1959).

9 ICRU, "Determination of absorbed dose in a patient irradiated by beams of X or gamma rays in radiotherapy procedures," ICRU Report 24, International Commission on Radiation Units and Measurements, Washington D.C. (1976).

10 AAPM Task Group 21 of the Radiation Therapy Committee, "A protocol for the determination of absorbed dose from high-energy photon and electron beams," Med. Phys. 10, 741-771 (1983). 
11 Task group 25 American Association of Physicist in Medicine, "Clinical electron beam dosimetry," Med. Phys. 18, 73-109 (1991).

12 International Atomic Energy Agency, Absorbed dose determination in photon and electron beams: An international code of practice (IAEA Technical Reports Series No. 277, Vienna, 1987).

13 Institute of Physics \& Engineering in Medicine \& Biology, "The IPEMB code of practice for the determination of absorbed dose for $\mathrm{x}$ - rays below $300 \mathrm{kV}$ generating potential $(0.035 \mathrm{~mm} \mathrm{Al}-4 \mathrm{~mm} \mathrm{Cu} \mathrm{HVL} ; 10-300 \mathrm{kV}$ generating potential). Institution of Physics and Engineering in Medicine and Biology," Phys. Med. Biol. 41 (12), 2605-2625 (1996).

14 Institute of Physics \& Engineering in Medicine \& Biology, "The IPEMB code of practice for electron dosimetry for radiotherapy beams of initial energy from 2 to $50 \mathrm{MeV}$ based on an air-kerma calibration. Institution of Physics and Engineering in Medicine and Biology," Phys. Med. Biol. 41 (12), 2557-2603 (1996).

15 International Atomic Energy Agency, Absorbed Dose Determination in External Beam Radiotherapy based on Absorbed-Dose-to-Water Standards: An international Code of Practice for Dosimetry, Draft V.7 (IAEA Technical Reports Series No. 398, Vienna, 2000).

16 P. R. Almond, P. J. Biggs, B. M. Coursey et al., "AAPM's TG-51 protocol for clinical reference dosimetry of high-energy photon and electron beams," Med. Phys. 26 (9), 1847-1870 (1999).

17 F. M. Khan, K. P. Doppke, K. R. Hogstrom et al., "Clinical electron-beam dosimetry: report of AAPM Radiation Therapy Committee Task Group No. 25," Med. Phys. 18 (1), 73-109 (1991).

18 J. W. Boag, "Ionization chambers," in The dosimetry of ionizing radiation, edited by K. R. Kase, B. E. Bjärngard and F. H. Attix (Academic Press, Orlando, 1985), Vol. 2, pp. 169-243. 
19 R. J. Schulz, P. R. Almond, G. Kutcher et al., "Clarification of the AAPM Task Group 21 protocol," Med. Phys. 13 (5), $755-759$ (1986).

20 M. S. Weinhous and J. A. Meli, "Determining Pion, the correction factor for recombination losses in an ionization chamber," Med. Phys. 11 (6), 846-849 (1984).

21 F. DeBlois, C. E. Zankowski, and E. B. Podgorsak, "Saturation current and collection efficiency for ionization chambers in pulsed beams," Med. Phys. 27 (5), 1146-1155 (2000).

22 C. E. Zankowski and E. B. Podgorsak, "Determination of saturation charge and collection efficiency for ionization chambers in continuous beams," Med. Phys. 25 (6), 908-915 (1998).

23 International Atomic Energy Agency, The use of plane parallel ionization chambers in high energy electron and photon beams: An international code of practice for dosimetry (IAEA Technical Reports Series No. 381, Vienna, 1997). 


\section{Chapter 5 \\ Experimental apparatus and techniques}

\section{Phantom-EMBEDdED EXTRAPOLATION CHAMBER}

\section{I.A. General design of the chamber}

The original phantom-embedded extrapolation chamber (PEEC) was designed by Zankowski and Podgorsak..$^{1-5}$ It has a parallel-plate geometry and the gap between the PEEC electrodes can be varied continuously from a fraction of a $\mathrm{mm}$ to about $1 \mathrm{~cm}$. The variation in electrode separation results in a variable sensitive air-volume which defines a variable Bragg-Gray cavity. The ionization charge collected for various PEEC air volumes produces ionization gradient data which can be used with a modified Spencer-Attix relation, Eq. (1-14), to determine the absorbed dose in Solid Water ${ }^{\mathrm{TM}}$ for megavoltage photon and electron beams. The PEEC chamber was originally made entirely of Solid Water ${ }^{\mathrm{TM}}$ material (model 457; Gammex-RMI, Middleton, WI) with a composition and radiological properties reviewed by various investigators. ${ }^{6-10}$ We modified the original PEEC design in three directions: the chamber piston was motorized to automatize the data collection, heterogeneous phantom materials were incorporated into the chamber to allow dose measurements in materials other than Solid Water ${ }^{\mathrm{TM}}$, and finally, the entrance window and polarizing electrode were modified in order to allow us to investigate the effect of the electrode materials and electrode thicknesses on the measured dose.

The PEEC schematics and its standard electrical operational diagram are shown

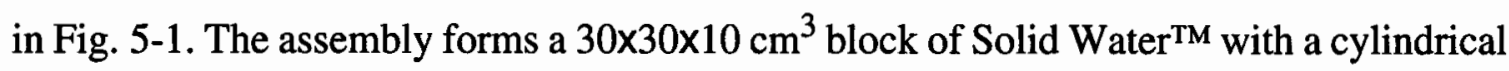
aperture of $7 \mathrm{~cm}$ diameter bored parallel to the direction of the beam in the center of the 
chamber. The cylinder bore is filled by a movable cylindrical piston also made of Solid Water ${ }^{\mathrm{TM}}$, shown in grey. The entrance window is a thin cylinder of $2 \mathrm{~mm}$ thickness and $9.7 \mathrm{~cm}$ diameter, while the top of the movable piston is a cylinder of $2 \mathrm{~cm}$ thickness and $7 \mathrm{~cm}$ diameter. Both pieces, shown in darker grey, are removable and available in Solid Water $^{\mathrm{TM}}$ as well as in bone-equivalent material (model SB3; Gammex-RMI, Middleton, WI). The principal characteristics of these two materials are summarized in Table 5-1.11 An aluminum frame, mounted on the bottom of the phantom body, holds the mechanical controls and electronic circuit of the piston positioning system.

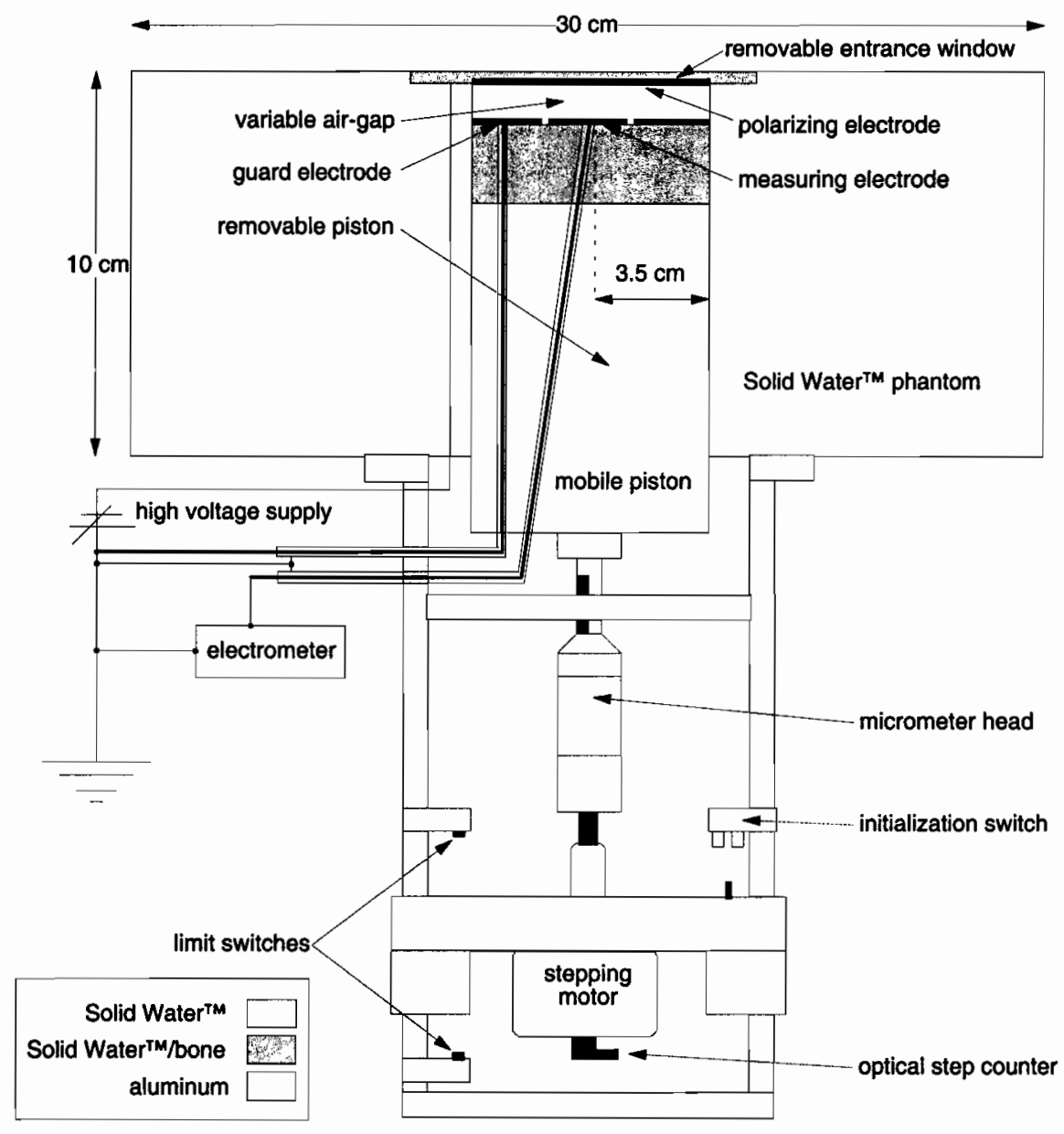

Figure 5-1. Schematic representation of the PEEC. The Solid Water ${ }^{\mathrm{TM}}$ material is shown in light grey and the removable parts, entrance window and piston, are shown in darker grey. 
A photograph of the PEEC is shown in Fig. 5-2 with the bone-equivalent material parts installed. A Lucite frame holds the whole chamber assembly and the various connectors for signal, high voltage, and stepping motor control, and also protects the piston electronic circuit and switches.

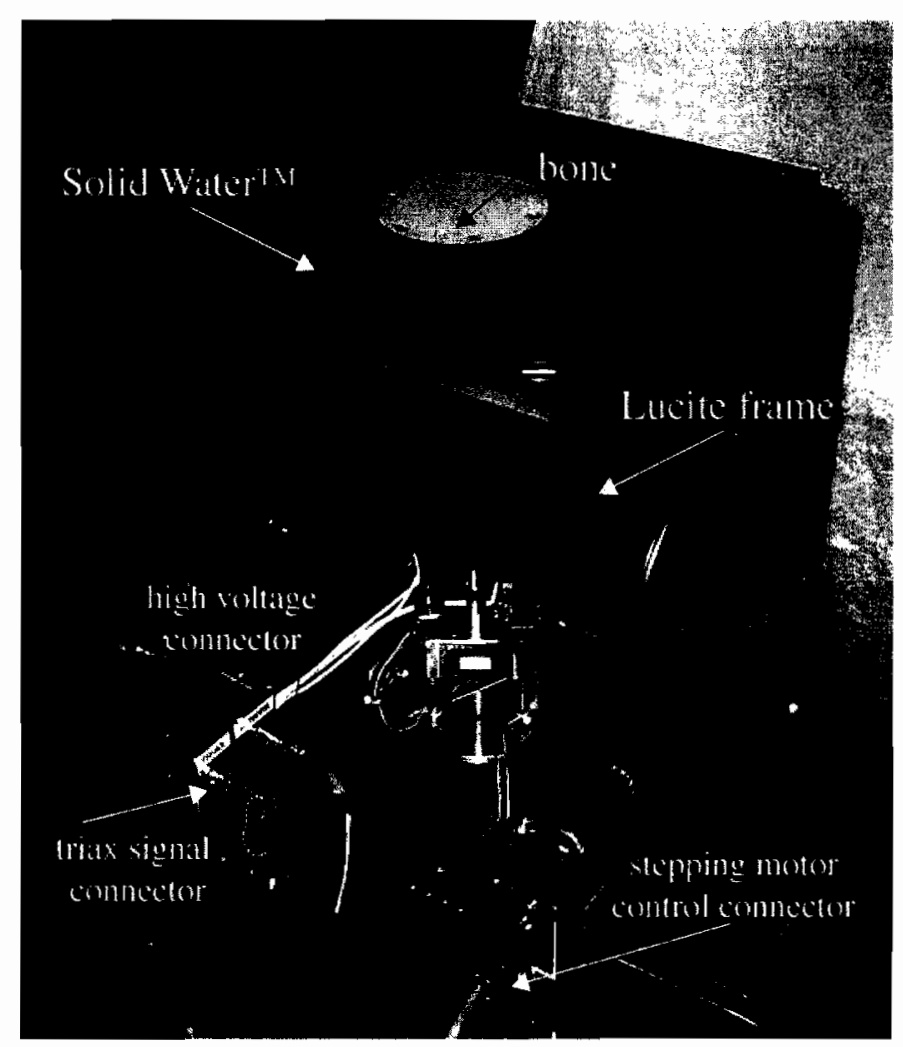

Figure 5-2. Photograph of the PEEC with the bone entrance window installed. The Lucite frame and the high voltage, signal and stepping motor connectors are also shown.

A thin layer ( $~ 0.05 \mathrm{~mm}$ ) of graphite dag is spray-painted directly onto the inside of the entrance window and on the top of the movable piston to form the polarizing, measuring, and guard electrodes, shown in black in Fig. 5-1. Polarizing electrodes are also available as thin $(0.025 \mathrm{~mm})$ metal sheets (steel 301 and brass) clamped onto the back of the entrance window by a circular piece of bone material, as shown in Fig. 5-3. The physical properties of the electrode materials are also summarized in Table 5-1. The sensitive variable air volume is defined by the separation between the two electrodes and the radius of the measuring electrode. The air gap is varied by moving the piston. 


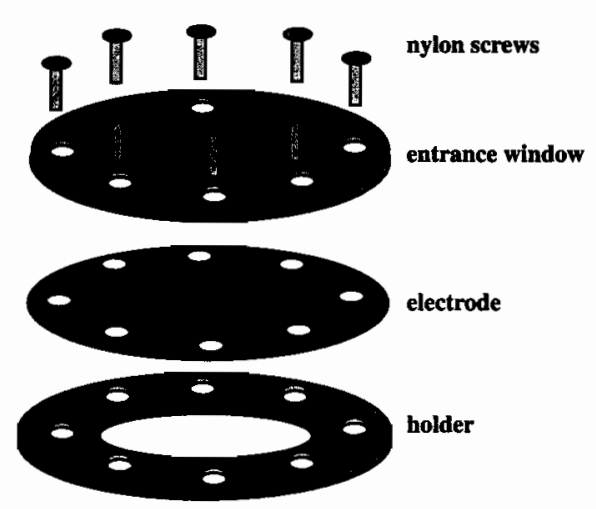

Figure 5-3. The entrance window with the polarizing electrode holder.

Table 5-1. Tissue-equivalent plastic and electrode material properties. $Z_{\text {eff }}$ is calculated from the formula given in Johns ${ }^{12}$.

\begin{tabular}{|c|c|c|c|c|c|c|}
\hline & Graphite & $\begin{array}{c}\text { Solid } \\
\text { Water }^{\mathrm{TM}} \\
\text { RMI model } \\
457\end{array}$ & $\begin{array}{c}\text { Hard Bone } \\
\text { RMI model } \\
\text { SB3 }\end{array}$ & Aluminum & $\begin{array}{c}\text { Steel } \\
\text { model } 301\end{array}$ & Brass \\
\hline$\rho\left(\mathrm{g} / \mathrm{cm}^{3}\right)$ & 1.7 & 1.035 & 1.840 & 2.702 & 7.880 & 9.210 \\
\hline$Z_{e f f}$ & 6 & 7.935 & 13.927 & 13.000 & 25.730 & 29.300 \\
\hline composition & $\%$ & $\%$ & $\%$ & $\%$ & $\%$ & $\%$ \\
\hline $\mathrm{H}$ & & 8.090 & 3.100 & & & \\
\hline $\mathrm{C}$ & 100.000 & 67.220 & 31.260 & & 0.150 & \\
\hline $\mathrm{N}$ & & 2.400 & 0.990 & & & \\
\hline $\mathrm{O}$ & & 18.84 & 37.570 & & & \\
\hline $\mathrm{Ca}$ & & 2.320 & 27.030 & & & \\
\hline $\mathrm{Cl}$ & & 0.130 & 0.050 & & & \\
\hline $\mathrm{Mn}$ & & & & & 2.000 & \\
\hline $\mathrm{Si}$ & & & & & 1.000 & \\
\hline $\bar{P}$ & & & & & 0.045 & \\
\hline $\mathbf{S}$ & & & & & 0.030 & \\
\hline $\mathrm{Cr}$ & & & & & 16.000 & \\
\hline $\mathrm{Ni}$ & & & & & 6.000 & \\
\hline $\mathrm{Fe}$ & & & & & 74.775 & \\
\hline $\mathrm{Al}$ & & & & 100.000 & & \\
\hline $\mathrm{Cu}$ & & & & & & 70.000 \\
\hline $\mathrm{Zn}$ & & & & & & 30.000 \\
\hline
\end{tabular}


Following a standard ionization chamber configuration, the measuring electrode, also called collecting electrode, of the chamber is connected to ground through a calibrated electrometer (model 35617; Keithley, Cleveland, $\mathrm{OH}$ ) and the guard ring is connected to ground directly. Two variable voltage power supplies (model 248; Keithley, Cleveland, $\mathrm{OH}$ and model 412B; John Fluke, Seattle, Washington) were used for the current and charge measurements. The model 248 and model $412 \mathrm{~B}$ provide a variable bias of up to $\pm 5000 \mathrm{~V}$ and up to $\pm 2100 \mathrm{~V}$, respectively.

The variable sensitive volume design of the PEEC allows a direct measurement of $\mathrm{d} Q / \mathrm{d} m$ for use in Eq. (1-14). Since the use of the modified Spencer-Attix equation does not require a determination of the sensitive air-mass for absolute dose measurement, no dose calibration factors, such as $N_{g a s}$ or $N_{D, w}^{C o}$, are required from standard laboratories. The guarded parallel-plate design of the PEEC eliminates the use of replacement correction factors $p_{r e p l}$ and $P_{g r}^{Q}$ associated with cylindrical ionization chambers and water phantoms, described in the AAPM TG-21 (Ref. \#13) protocol and in the AAPM TG-51 (Ref. \#14) protocol for the calibration of high-energy photon and electron beams. This assumption was verified experimentally by comparing the PEEC response with that of a standard Farmer chamber and theoretically with Monte Carlo calculations through calculating the fluence correction factors for the PEEC chamber geometry (Chapter 6) over a range of electrode separations between 0.5 to $5 \mathrm{~mm}$. There is also no need for correction factors to account for differences in the wall and medium material, since the PEEC is embedded within the phantom material; a very important PEEC feature considering that no correction factors are available for cylindrical chambers in heterogeneous phantoms. Moreover, to minimize the amount of backscatter from extracameral non-phantom materials, all metallic components of the PEEC, principally the motion-controlled piston assembly, were located at least 10 $\mathrm{cm}$ from the chamber sensitive volume. The piston housing and the stepping motor housing, which are mounted at the bottom of the phantom body, were made of aluminum $(Z=$ 13) to produce minimal backscatter into the chamber sensitive air volume.

The use of the bone-equivalent entrance window and piston top approximates a bone-equivalent phantom and allows a direct measurement of the dose-to-bone. It is assumed that all backscatter radiation contributing to the dose is produced in the bone material and that lateral scatter is negligible in the chamber geometry. These assumptions were 
verified with Monte Carlo calculations by simulating the chamber as if it was made entirely of bone material and comparing the results to those measured with the actual experimental bone/Solid Water ${ }^{\mathrm{TM}}$ design (Chapter 8 ).

Finally, we found that the presence of the graphite electrodes in the bone phantom perturbs the beam fluence in a non-negligible manner and a correction factor must be applied in order to obtain the dose to the bone material. The electrode perturbation effect is analysed in detail in Chapter 8 where the effect produced by various electrode materials (graphite, steel 301, brass, and aluminum) and thicknesses is studied as a function of beam energy by means of measurements as well as Monte Carlo simulations. We also verified that the graphite electrodes had no effect on the measured dose in the case of PEEC measurements performed in Solid Water ${ }^{\mathrm{TM}}$.

\section{I.B. Electrode construction}

The electrode design for the phantom-embedded extrapolation chamber was studied in detail by Zankowski. ${ }^{3}$ The measuring and polarizing electrodes are designed to be interchangeable easily. The component that holds the collecting electrode consists of a $7 \mathrm{~cm}$ diameter, $0.5 \mathrm{~cm}$ thick disc of Solid Water ${ }^{\mathrm{TM}}$ for measurements in Solid Water ${ }^{\mathrm{TM}}$ and of a $7 \mathrm{~cm}$ diameter, $2 \mathrm{~cm}$ thick disc of bone-equivalent material for measurements in bone phantom. The components are attached to the piston with nylon screws. Small spring-loaded gold-plated pins are pushed through small holes, so that the flat closed surfaces of the pins are levelled with the disc surface on which the electrode is spray-painted. A lathe was used to etch a small circular groove into the graphite layer to produce two independent conducting surfaces: the collecting electrode and the guard electrode. There is one pin for the central collecting electrode and one pin for the guard electrode. The guard electrode prevents leakage currents from reaching the measuring electrode and also defines the chamber sensitive volume. Small pins, welded to triaxial shielded cables, connect tightly to the electrode pins to form a reliable electrical connection.

The entrance window is formed with $9.7 \mathrm{~cm}$ diameter, $4 \mathrm{~mm}$ thick discs made of Solid Water or bone. The central area of the discs is reduced to a $2 \mathrm{~mm}$ thickness over a 7.3 
$\mathrm{cm}$ diameter onto which the polarizing electrode is spray-painted. The entrance window covers the cylindrical aperture and is attached to the top portion of the phantom body with nylon screws. The high-voltage connection to the polarizing electrode is accomplished with a spring-loaded brass pin, touching the polarizing electrode surface.

The electrode surfaces consist of either thin uniform layers of graphite dag (Aquadag; Acheson colloids (Canada) Limited, Ontario), spray-painted with a pressurized airbrush (Eclipse; Iwata, Japan) directly onto the tissue-equivalent material surface or various metal sheets clamped onto the entrance window, as illustrated in Fig. 5-3.

The graphite dag has a low atomic number $(Z=6)$ and is a good conductor for use in radiation dosimetry with Solid Water ${ }^{\mathrm{TM}}$ material. The dag is applied progressively as thin $\sim 10 \mu \mathrm{m}$ layers, each sanded and polished to ensure uniform deposition of the graphite and to remove surface irregularities resulting in the spray-painting process. The electrode should have a resistance below $30 \Omega$ across the electrode diameter corresponding to a thickness of graphite of less than $0.05 \mathrm{~mm}$.

Metal sheets we also used for polarizing electrodes and are made of thin $(0.025 \mathrm{~mm})$ steel 301 and brass material (Shim and Metal Processing, Toronto, Canada), with exact composition shown in Table 5-1. Their resistance is typically $<10 \Omega$ across the diameter of the electrode. They are first glued onto the entrance window with traces of highly compressible lithium base grease (Lubetech Petroleum, Chicago, IL) and then clamped by an annulus-shaped piece of bone material, as shown in Fig. 5-3. This system allows an easy interchange of the polarizing electrodes in studies of electrode material effects on dose measurements.

\section{COMPUTER-CONTROLLED SYSTEM}

The operation of the PEEC was automated to simplify the measurement process. A personal computer (486 PC compatible) was used to remotely control the operation of the PEEC setup. The various interfaces and components of the PEEC controls are shown schematically in Fig. 5-4. Computer programs were written in Basic language (QuickBasic $^{\mathrm{TM}}$ v.4.5; Microsoft, Redmond, WA) to give the user an interface for controlling both the 
in-house-built stepping motor assembly which varies the sensitive volume and a GPIB (IEEE Std 488.2-1992; Institute of Electrical and Electronic Engineers, New York, NY) interface which controls all standard IEEE-488 instruments. The programs are menu-driven and allow the user to visualize and analyze the results on screen and also to write them to text files for further analysis.

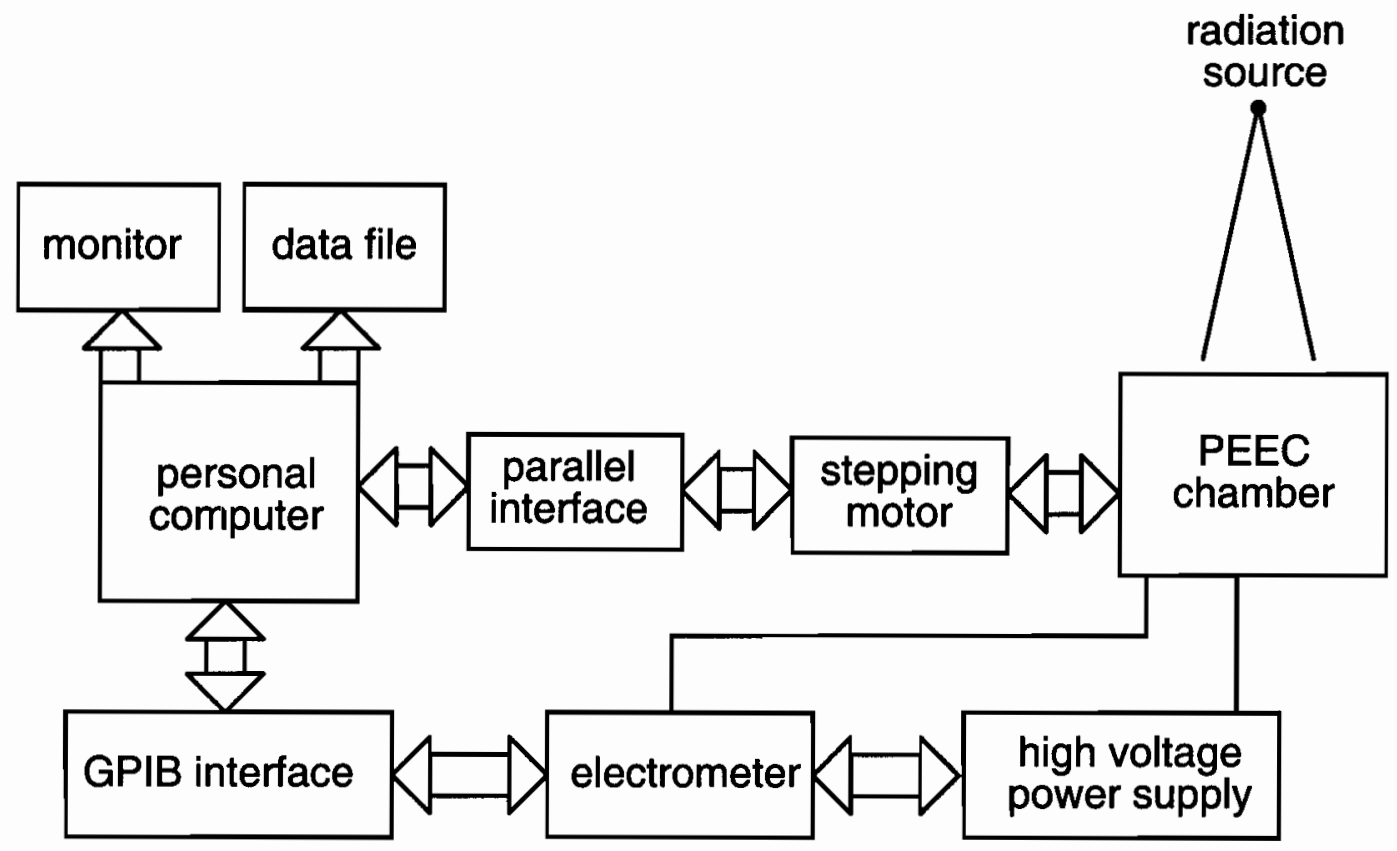

Figure 5-4. Schematic diagram of the PEEC computer control system.

\section{II.A. GPIB interface}

A miniature serial to IEEE 488 controller (Micro 488/p-901 rev 1.0; IOtech Inc., Cleveland, $\mathrm{OH}$ ) is used to convert RS-232 serial port commands from the host computer into an IEEE-488 bus "talker", "listener", and "controller" for up to 8 IEEE-488 instruments on a daisy chain cable at a maximum distance of 50 feet. Both the high voltage power supply (Keithley 248) and the electrometer (Keithley 35617) are controlled on the IEEE bus. A photograph of the computer-control instruments is shown in Fig. 5-5. 
Depending on the type of measurements performed, various custom programs were used to control the instruments. The basic set of the IEEE-488 commands used for interfacing with various instruments through the microcontroller is given in Appendix 1.

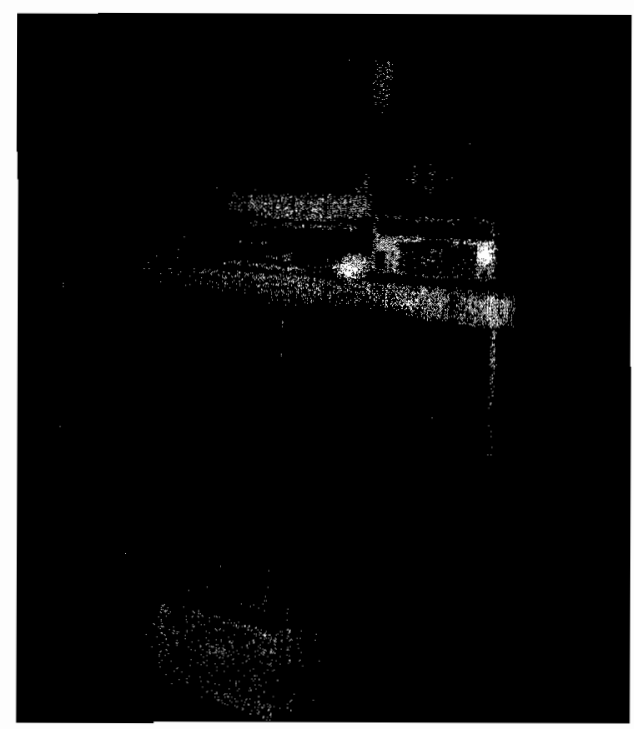

Figure 5-5. Computer system to remotely control the PEEC: a 486 personal computer controls the electrometer (Keithley 35617 ) the high voltage power supply (Keithley 248) on the IEEE-488 interface and the stepping motor interface through the parallel port.

\section{II.B. Piston remote control}

The piston is moved using a Quick Basic ${ }^{\mathrm{TM}}$ computer program that controls a stepping motor, which is directly connected to the micrometer head which in turn is attached to the bottom of the piston through a ball-bearing mechanism. The stepping motor holder sits on two vertical cylindrical tracks on which it slides as the motor shaft rotates the micrometer in one direction or the other. An aluminum box contains the printed circuit and power supply for the stepping motor. The motor control box is connected to the computer parallel port through a standard parallel cable. The chamber controls for the stepping motor are connected to the motor control box through a 9-wire, shielded cable that is 10 meters long to allow the connection between the chamber which is in the treatment room and the 
control system which is in the linac control room. The movement of the motor holder is electronically limited by two optical limit switches, shown schematically in Figure 5-1. These limit switches can be moved along the cylindrical tracks and tightened into appropriate positions with a set screw. The position of switches is chosen to safely operate the motor by avoiding electrode collision.

The motor rotates through one step when receiving a sequence of pulses. The precise position of the motor within one rotation is monitored constantly by an optical step counter attached to the shaft of the motor. If the monitored motor position is incorrect the program stops and an error message is given to the user. The stepping motor undergoes 200 steps per revolution and one revolution of the micrometer corresponds to a displacement of $0.5 \mathrm{~mm}$, therefore the electrode separation can be controlled theoretically up to a precision of $2.5 \mu \mathrm{m}$. The motor is first initialized to a "zero" position corresponding to a relative displacement $z=0$ of the electrode. This position is reached when the motor holder hits the optical initialization switch. This switch is also movable along one of the cylindrical tracks and fastened with a set screw to select the minimal operational distance between the electrodes which is selected to correspond to a separation $d$ of $\sim 0.5 \mathrm{~mm}$. The control program allows the user to move the electrodes to any relative position $z$ up to a maximum distance, defined by the lower limit switch which is set at $d \approx 10 \mathrm{~mm}$. The complete schematic of the electronic circuit used to control the stepping motor through the parallel port as well as an example program in QuickBasic ${ }^{\mathrm{TM}}$ to control the motor are given in Appendix 2.

The linearity of the relation between the position requested by the program and the micrometer reading, as well as the reproducibility of the motion, were verified extensively. Figure 5-6 shows the position of the micrometer as a function of the software-requested position. The relative agreement on the motion is nearly perfect with a slope of $1.000 \pm 0.001$, indicating that the estimate on the error position due to the finite steps of the motor of $2.5 \mu \mathrm{m}$ is acceptable. However, a small deviation from linearity is observed for very low positions of the micrometer which are actually less than 0 on the micrometer readout. To avoid this problem the chamber was operated relatively far from that position. Reproducibility of relative positions, when going in one direction of movement is excellent; however, if one has to go back in position and then forward, a small hysteresis effect on the motion of the micrometer due to some "play" in the mechanism may 
produce small differences in readout. To avoid the hysteresis problem, the chamber was always operated by moving the piston in the same direction. In situations that required a return travel, a new initialization to the $z=0$ position was made.

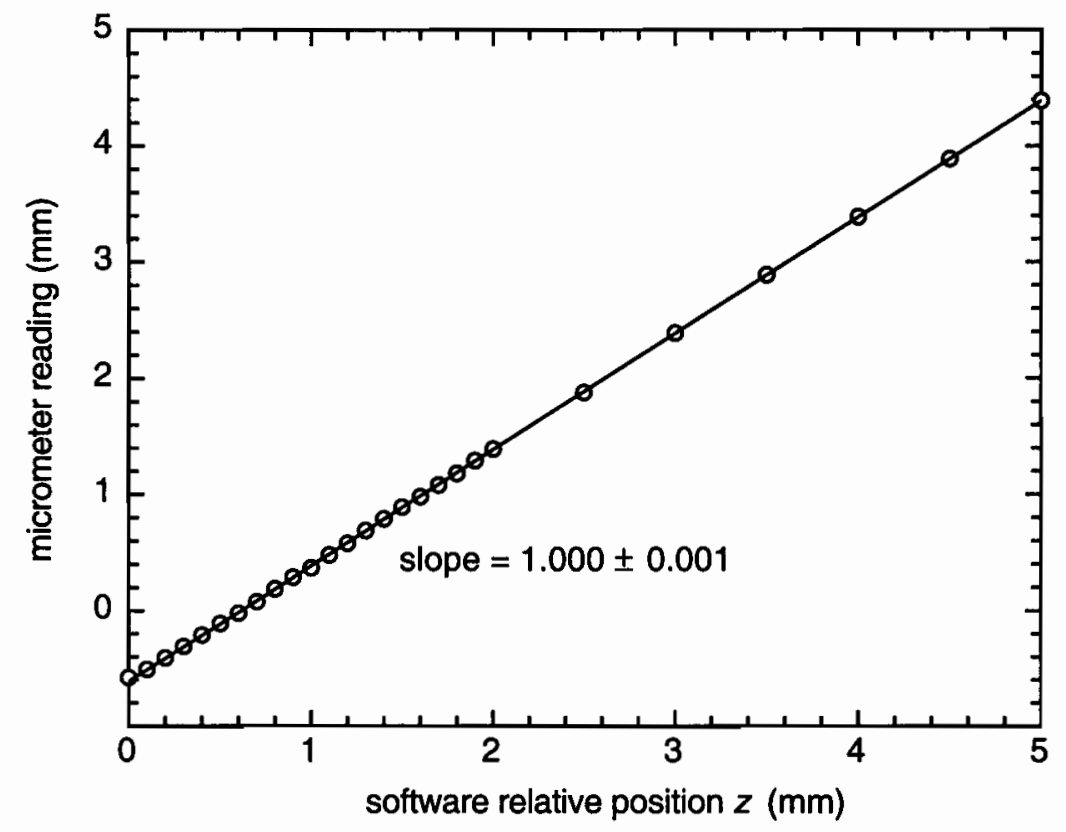

Figure 5-6. Relative position of the micrometer as a function of the software requested position. The agreement is excellent except for the first point at which the micrometer is positioned at its lower limits.

\section{II.C. Electrode area determination}

Successful determination of absolute dose with the PEEC depends on the precise knowledge of the effective area $A$ of the measuring electrode. The physical measurement of the area of the measuring electrode might differ slightly from the effective electrode area because of the finite width of the groove which separates the measuring and guard electrodes and the uncertainty in the determination of the electric field lines in the groove. An electrical method for the determination of area $A$ is therefore preferred.

The capacitance $C$ of a parallel plate ionization chamber, with sufficiently large guard and collecting electrodes, is given by: 


$$
C=\frac{\Delta Q}{\Delta V}=\varepsilon_{o}\left(\frac{A}{d}\right)
$$

where $\varepsilon_{\mathrm{o}}$ is the electrical permittivity of vacuum $\left(8.854 \times 10^{-12} \mathrm{~F} / \mathrm{m}\right), d$ the separation between the electrodes, and $\Delta Q$ the variation in charge measured by the electrometer for a change in polarizing voltage $\Delta V$. Since $d$ is not known accurately, the capacitance $C$ of the extrapolation chamber is measured as a function of the relative electrode separation $z$ and the collecting electrode area $A$ is determined from a plot of the inverse capacitance $1 / C$ vs. $z$ which results in a straight line with a slope of $\varepsilon_{0} A^{-1}$, as shown in Fig. 5-7. The $x$-intercept value $(z \approx-0.65 \mathrm{~mm}$ ) could be used to calibrate the absolute electrode separation in the computer control program; however, the use of Eq. (1-14) for dose measurements makes this piece of information superfluous. The procedure for area determination was repeated for both polarities and before every use of the chamber, and the average of all obtained values is $4.61 \pm 0.01 \mathrm{~cm}^{2}$ for the Solid Water ${ }^{\mathrm{TM}}$ PEEC graphite electrode and $8.019 \pm 0.015 \mathrm{~cm}^{2}$ for the bone PEEC graphite electrode.

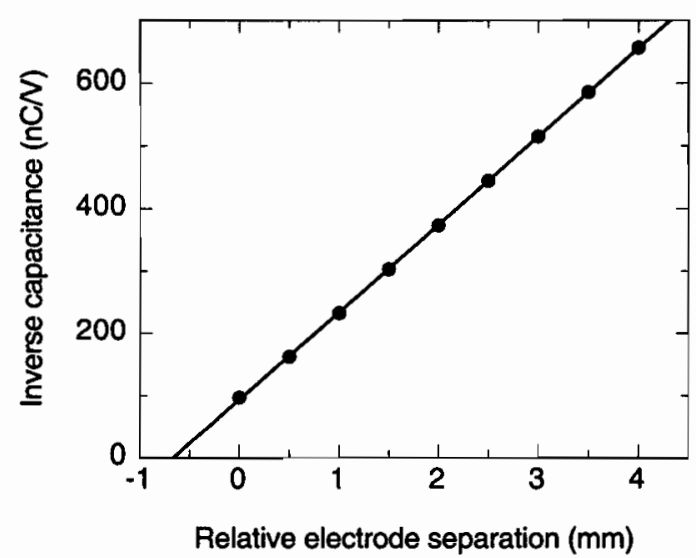

Figure 5-7. Inverse capacitance as a function of relative electrode separation measured with the extrapolation chamber with the bone material in place. The slope of the least-squares fit to measured data is $\left(\varepsilon_{0} A\right)^{-1}$ from which the effective collecting electrode area $A$ is calculated. 


\section{II.D. Ionization gradient determination}

The ionization gradient $\mathrm{d} Q / \mathrm{d} z$ of Eq. (1-14) is determined from a measured $Q$ vs. $z$ relationship. A typical example of this relationship in the relative electrode separation range from about 0 to $3.5 \mathrm{~mm}$ is shown in Figure 5-8 for the $9 \mathrm{MeV}$ electron beam. The chamber readings were corrected for the chamber recombination losses and the saturation charges, and represent the mean values for positive and negative chamber polarities. A least-squares fit to the measured data in this range yields a straight line.

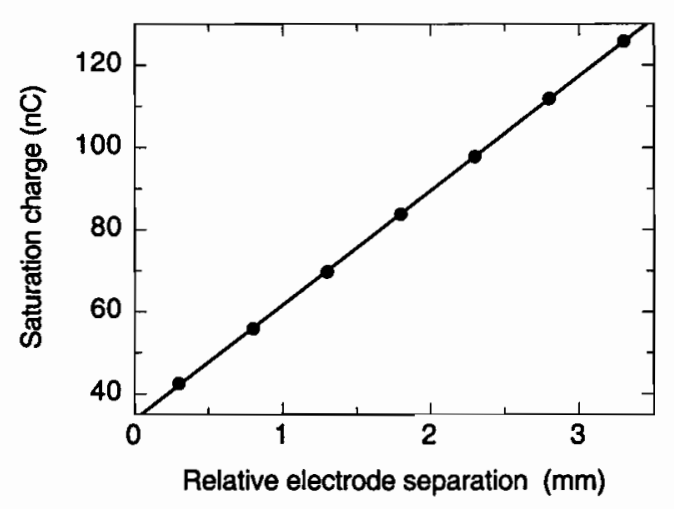

Figure 5-8. Saturation charge as a function of the relative electrode separation measured with the extrapolation chamber for a $9 \mathrm{MeV}$ electron beam and the bone piston top and entrance window.

\section{II.E. Linearity of the piston control system}

The use of the area determination through a capacitance measurement technique allows further investigation on the linearity of the remote control system. The area $A$ of the collecting electrode was determined from the measured capacitance of 100 different electrode separations, ranging from $\sim 1$ to $4 \mathrm{~mm}$. The capacitance $C$ at each electrode separation was obtained from 100 different measured $\Delta Q / \Delta V$ points, with $\Delta V$ ranging from 4 to 400 $\mathrm{V}$ and a reference voltage of $\pm 50 \mathrm{~V}$ depending on the polarity. The area determination was 
carried out for positive and negative electrode bias polarities following the method described above using Eq. (5-1). In order to evaluate any possible systematic positioning errors introduced by the piston control system, the determined area was used to calculate a capacitance value $C^{\prime}$ at each gap of the previous measurement point by means of Eq. (5-1). The percentage residuals between $C$ and $C^{\prime}$ are plotted in Fig. 5-9.

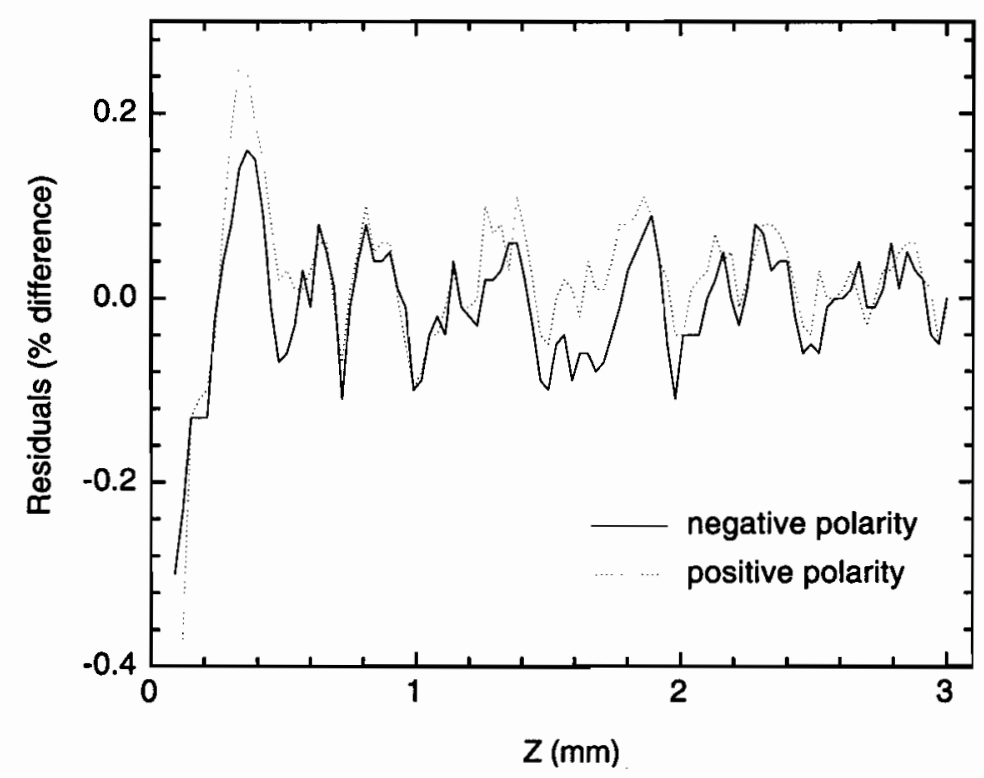

Figure 5-9. Plot of the residuals between $C$ and $C^{\prime}$.

Very small variations, within $0.2 \%$, are noticed when plotting the percentage residuals between the measured capacitance $C$ at each point and the expected capacitance $C^{\prime}$ calculated from the area determined using all points. A periodic pattern is observed and the pattern is similar for both polarities indicating a slight non linearity between each step of the stepping motor. Moreover, the period is equal to the motion corresponding to a full turn of the stepping motor $(0.5 \mathrm{~mm})$. This behavior is possibly produced by mechanical resistance to the piston motion or by an inherent non-linear motion of the micrometer screw. However, the electrode positioning error is essentially negligible and will not be considered. 


\section{II.F. PEEC chamber leakage measurement}

In order to verify that leakage current does not adversely influence our results, chamber leakage current was measured as a function of the PEEC polarizing voltage. For a typical PEEC electrode spacing of $2.5 \mathrm{~mm}$, the polarizing voltage was first increased from 50 and $800 \mathrm{~V}$ (scan up) and then decreased (scan down) from 800 to $50 \mathrm{~V}$ and the cycle was then repeated. 1000 current readings were acquired at each polarizing voltage to obtain a good average of the leakage current at a particular voltage. The steps in voltage were $10 \mathrm{~V}$. Results are shown in Fig. 5-10.

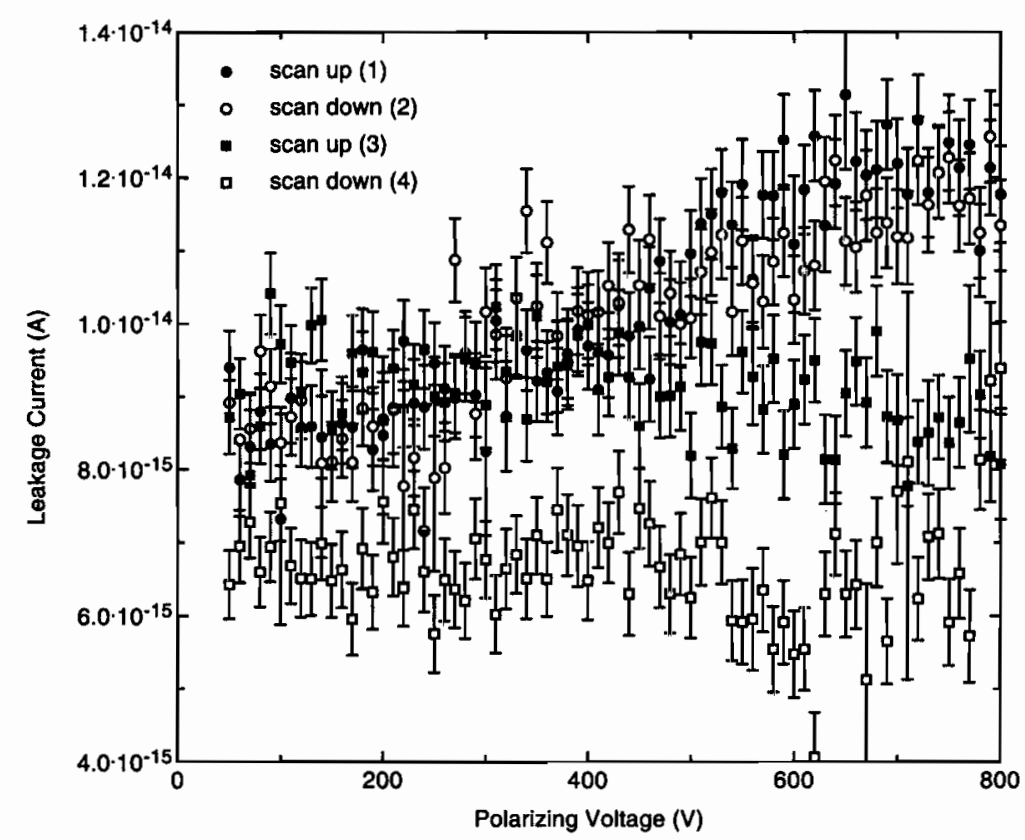

Figure 5-10. PEEC chamber leakage current as a function of polarizing voltage.

While the first scan produces an increase in the leakage current with voltage, subsequent scanning stabilizes the current at the initial low value irrespective of the polarizing voltage. We thus conclude that the leakage currents, typically on the order of $10^{-14} \mathrm{~A}$, can be neglected when measuring the radiation signal with the PEEC, since dosimetric readings measured with the $\mathrm{PEEC}$ are typically $10^{-9} \mathrm{~A}$, i.e., five orders of magnitude higher than the leakage level of $10^{-14} \mathrm{~A}$. 


\section{II.G. Calibration of electrometer and power supplies}

In order to determine an absolute dose reading, it is important to measure the collected charge accurately. The response of the 35617 Keithley electrometer was verified with a calibrated picoampere current source (model 261; Keithley, Cleveland, Ohio) with a calibration factor traceable to a standards laboratory (National Research Council, Ottawa, Canada). The current source was connected to the input of the relay switch which had its output connected directly to the electrometer. The current source was set to deliver $\pm 1.000 \mathrm{nA}$ during a programmed interval of 100.0 seconds to produce a total output of $100.0 \mathrm{nC}$ to the electrometer. A universal timer which includes a relay triggered by an electronic pulse-counter was used for the timing device. The pulse counter is driven by a synchronized internal clock, accurate to better than $\pm 1 \mathrm{~ms}$. The error on the timing circuit is less than $2 \mathrm{~ms}$, therefore negligible over a $100 \mathrm{~s}$ range. The electrometer was found to be precise to within $\pm 0.02 \%$, which is well below the maximum acceptable error in absolute dose measurement.

Both voltage power supplies were verified to deliver voltages within their specifications with a high precision voltmeter (model 2000; Keithley, Cleveland, Ohio) following the calibration procedure described in their respective manuals. The error on the bias voltage was well below the error that would affect the current measurements, since near saturation the current is varying very slowly with a change of voltage.

\section{II.H. Collection potential}

Ionic recombination normally plays a non-negligible role in radiation dosimetry. Every electrode separation of the PEEC has a different recombination correction factor for each different megavoltage radiation beam energy and type (continuous photon, pulsed photon, and pulsed electron beam). Following careful measurement of saturation curves, a deviation from the Boag theory for continuous and pulsed beams ${ }^{15-18}$ at high bias voltages was observed and a modified theory is proposed for both continuous ${ }^{1,19}$ and pulsed radiation ${ }^{20}$ beams to account for radiation induced leakage. The choice of optimum polarizing potential range in which the chamber should be operated is selected from the range of 
collection efficiencies $f$ in which the saturation curves follow Boag theory, i.e., in the range $0.7 \leq f \leq 0.9$. An electric field strength of $200 \mathrm{~V} / \mathrm{mm}$ is sufficient to operate the PEEC in the optimum charge collection region for all electrode separations, as demonstrated by measuring full saturation curves ${ }^{1}$ (see Chapter 7 ).

\section{THERMOLUMINESCENT DOSIMETRY}

For experimental verification of dose measurements with the PEEC thermoluminescent dosimetry (TLD) techniques were used in addition to Farmer type ionization chambers. The TLD procedures consisted of a TLD reader (model 3500; Harshaw Chemical Company, Solon, $\mathrm{OH}$ ) and dosimeters in the form of TLD chips with dimensions of

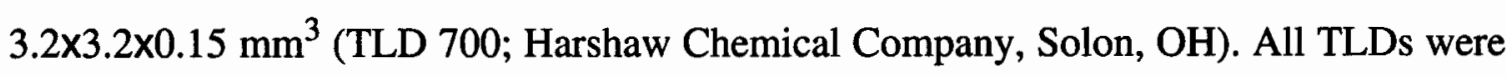
individually calibrated at each energy at the dose maximum in Solid Water before and after depth dose measurements. At least six TLDs were used to determine the dose at each depth. The reproducibility expressed as the sample standard deviation on a dose measurement at a given depth amounts typically to $0.5 \%$. The average TLD reading was converted to dose to the material of interest (bone) by using the Spencer-Attix stopping power ratio of the material to TLD determined in a separate calculation with Monte Carlo techniques.

\section{Measurement OF dose-to-Bone With the PEEC}

The phantom-embedded extrapolation chamber was used to determine the absorbed dose to phantom material from various high energy photon and electron beams which are in clinical use at the McGill University Health Center (MUHC) in Montreal. Dose measurements were carried out in Solid Water ${ }^{\mathrm{TM}}$ and in bone-equivalent material. The dose measurements made in Solid Water ${ }^{\mathrm{TM}}$ were compared to measurements conducted using calibrated Farmer-type chambers in Solid Water ${ }^{\mathrm{TM}}$ as well as to Monte Carlo calculations. The dose measurements made in bone-equivalent material were compared to doses determined with Monte Carlo calculations and with TLD measurements. 
Dose measurements were carried out with a cobalt-60 gamma source (T-780; AECL, Ottawa Ontario), with x-ray beams in the energy range from 6 to $18 \mathrm{MV}$, and with electron beams in the nominal energy range from 9 to $15 \mathrm{MeV}$. All high-energy $\mathrm{x}$-ray beams were provided by a Clinac- 2300 linac (Varian; Palo Alto, CA) and the electron beams were provided by a Clinac-2300 and by a Clinac-18 linac (Varian; Palo Alto, CA). Output measurements were measured in phantom at a depth of $5 \mathrm{~g} / \mathrm{cm}^{2}$ for photon beams and at the depth of dose maximum $d_{\max }$ for the given electron beam. Percentage depth dose curves for both Solid Water ${ }^{\mathrm{TM}}$ and bone-equivalent material were measured and calculated by Monte Carlo simulations for all beams (see Chapter 6). The reference point of measurement for the PEEC is the proximal surface of the polarizing electrode, i.e., the surface of the graphite dag layer on the entrance window. The desired depths of measurement were achieved by placing slabs of the desired material on top of the chamber and accounting for the thickness of the entrance window $(2 \mathrm{~mm})$.

Measurements with the PEEC were carried out for various electrode separations and bias voltages chosen to satisfy the Bragg-Gray conditions. Electric fields were on the order of $\sim 200 \mathrm{~V} / \mathrm{mm}$, with 1.5 to $3.0 \mathrm{~mm}$ electrode separation as suggested by Monte Carlo calculations (see Chapter 8). For each particular electrode separation, ionization values $Q$ were measured for at least 3 different bias voltages $V$ selected such that the collection efficiency was in the $0.7<f<0.9$ range. Those values were plotted as $1 / \mathrm{Q}$ vs. $1 / \mathrm{V}$ or $1 / \mathrm{Q}$ vs. $1 / \mathrm{V}^{2}$, for pulsed and continuous beams, respectively, and a linear extrapolation to the y-intercept $1 / Q_{\text {sat }}$ of the data was made to obtain the saturation charge $Q_{\text {sat }}$, hence correcting the readings for charge recombination (see Chapter 7). At least four of these $Q_{\text {sat }}$ values were obtained and plotted as a function of the relative electrode separation $z$. The slope of this relationship yields the $\mathrm{d} Q / \mathrm{d} z$ value used in the dose equation Eq. (1-14). All ionization measurements were corrected for temperature and pressure and repeated at least 4 times resulting in a standard error that is less than $0.1 \%$.

Values of the mean restricted stopping power $(\bar{L} / \rho)_{\text {air }}^{\text {med }}$ required in Eq. (1-14) were calculated with Monte Carlo techniques using realistic beam modelling with SPRRZnrc, as described in Chapter 6. 
Correction factors were applied to account for the electrode material and scatter deficit of the bone PEEC. Chapter 8 presents the dose determination results as well as the electrode effect and scatter deficit study.

\section{SUMMARY}

The phantom-embedded extrapolation chamber (PEEC) is a variable air-volume parallel plate ionization chamber that forms part of a Solid Water ${ }^{\mathrm{TM}}$ water-equivalent phantom. Replacing the entrance window and the top of the chamber piston by similar components made of bone-equivalent material, we approximate a bone-equivalent phantom. Relative electrode separations can be varied from $0.5 \mathrm{~mm}$ to $10 \mathrm{~mm}$ with a precision of \pm 2.5 $\mu \mathrm{m}$ by means of a stepping motor, remotely controlled by a personal computer. The IEEE-488 measurement instruments are also controlled by the computer. The PEEC design minimizes unwanted backscatter due to non-phantom materials through limiting the use of metal near the sensitive air volume. The electrode area, required for dose calculation, is determined from a simple capacitance measurement to a precision of better than $0.20 \%$. 


\section{References}

1 C. E. Zankowski and E. B. Podgorsak, "Determination of saturation charge and collection efficiency for ionization chambers in continuous beams," Med. Phys. 25 (6), 908-915 (1998).

2 C. E. Zankowski, S. Vatnitsky, J. Siebers et al., "Proton beam output measurement with an extrapolation chamber," Med. Dosim. 23 (4), 288-291 (1998).

3 C. E. Zankowski, "Calibration of photon and electron beams with an extrapolation chamber," Ph.D. Thesis, McGill University, 1997.

4 C. E. Zankowski and E. B. Podgorsak, "Ionization gradient chamber in absolute photon and electron dosimetry," Radiology and Oncology (Slovenia) 30, 138-141 (1996).

5 C. E. Zankowski and E. B. Podgorsak, "Calibration of photon and electron beams with an extrapolation chamber," Med. Phys. 24 (4), 497-503 (1997).

6 C. Constantinou, F. H. Attix, and B. R. Paliwal, "A solid water phantom material for radiotherapy x-ray and gamma-ray beam calibrations," Med. Phys. 9 (3), 436-441 (1982).

7 C. Constantinou, N. F. Kember, G. Huxtable et al., "Physical measurements with a high-energy proton beam using liquid and solid tissue substitutes," Phys. Med. Biol. 25 (3), 489-499 (1980).

8 A. K. Ho and B. R. Paliwal, "Stopping-power and mass energy-absorption coefficient ratios for Solid Water," Med. Phys. 13 (3), $403-404$ (1986). 
9 A. K. Ho, B. R. Paliwal, and F. H. Attix, "Charge storage in electron-irradiated phantom materials," Med. Phys. 13 (1), 99-100 (1986).

10 C. S. Reft, "Output calibration in solid water for high energy photon beams," Med. Phys. 16 (2), 299-301 (1989).

11 Gammex RMI (Private communications).

12 Harold Elford Johns and John Robert Cunningham, The physics of radiology, 4th ed. (Charles C. Thomas, Springfield, Ill., U.S.A., 1983).

13 AAPM Task Group 21 of the Radiation Therapy Committee, "A protocol for the determination of absorbed dose from high-energy photon and electron beams," Med. Phys. 10, 741-771 (1983).

14 P. R. Almond, P. J. Biggs, B. M. Coursey et al., “AAPM's TG-51 protocol for clinical reference dosimetry of high-energy photon and electron beams," Med. Phys. 26 (9), $1847-1870$ (1999).

15 J. W. Boag, "Ionization measurements at very high intensities I. Pulsed radiation beams," Br. J. Radiol. 23, 601-611 (1950).

16 J. W. Boag, "The saturation curve for ionization measurements in pulsed radiation beams," Brit. J. Radiol. 25, 649-650 (1952).

17 J. W. Boag, "Ionization chambers," in The dosimetry of ionizing radiation, edited by K. R. Kase, B. E. Bjärngard and F. H. Attix (Academic Press, Orlando, 1985), Vol. 2, pp. 169-243. 
18 J. W. Boag and J. Currant, "Current collection and ionic recombination in small cylindrical ionization chambers exposed to pulsed radiation,” Br. J. Appl. Phys. 53, 471-478 (1980).

19 C. L. Yang, D. W. Rogers, K. R. Shortt et al., "Ion recombination in ion chambers in continuous radiation," proceedings of the $45^{\text {th }}$ scientific meeting of the COMP, Sherbrooke, June 16-19, 23-25 (1999).

20 F. DeBlois, C.E. Zankowski, and E. B. Podgorsak, "Saturation current and collection efficiency for ionization chambers in pulsed beams," Med. Phys. 27 (5), 1146-1155 (2000). 


\section{Chapter 6}

\section{Monte Carlo calculations}

\section{INTRODUCTION}

The Monte Carlo method provides a numerical solution to a problem obtained through modelling objects interacting together or with their environment following simple rules of interactions. The first historical reference to the Monte Carlo method is from Comte de Buffon ${ }^{1}$ in 1777, who evaluated the probability of tossing a needle onto a ruled sheet with a Monte Carlo-like method. The needle of length $L$, tossed randomly on a plane ruled with parallel lines of distance $d$ apart $(d>L)$, has a probability $p=2 L / \pi d$ to fall on a line. In 1886 , Laplace $^{2}$ proposed a similar procedure to determine the value of $\pi$ that can be illustrated by the following situation: darts are randomly thrown onto a figure composed of a square of side $d$ which encloses a quarter circle of radius $d$. The ratio of hit events within the quarter circle to the total number of events slowly converges to $\pi / 4$. Several other early applications of Monte Carlo methods are reviewed by Kalos and Whitlock ${ }^{3}$.

Monte Carlo methods were first suggested as a modern research tool during the second world war for the development of thermonuclear weapons ${ }^{4,5}$ when Ulam and von Neumann performed direct simulation of the probabilistic problems concerned with random neutron diffusion in fissile material ${ }^{6}$. The two physicists were the first to associate the name Monte Carlo to that type of numerical technique by reference to the famous city in the Monaco principality and are therefore considered pioneers in the development of modern Monte Carlo techniques. Subsequently the Monte Carlo method was applied by Goldberger ${ }^{7}$ to study nuclear disintegrations produced by high-energy particles. Later, Wilson ${ }^{8}$ in 1952 was the first to apply Monte Carlo techniques to study particle shower production, which served as a basic for Monte Carlo methods applied in the field of medical 
radiation physics. The first reports of digital computer-based Monte Carlo methods are from Butcher and $\mathrm{Messel}^{9}$ and, independently, Varfolomeev and Svetlolobov ${ }^{10}$.

Monte Carlo calculations are often used to model probabilistic events or processes. The core of a Monte Carlo simulation is the accurate knowledge of the probability distributions that govern the phenomena that one desires to model. The state of the system is first defined, and then updated at each step of the calculation, by randomly sampling the probability distributions that apply to the specific state of the system. The random sampling is performed with the help of a computer pseudo-random number generator (RNG) code that simulates the stochastic nature of the interactions. The evolution of a system from its initial state to its final state is called its history. As the history is performed some physical microscopic and macroscopic quantities can be calculated. Summing those quantities over a large number of histories gives average values and the standard deviations of those quantities. These values can be verified or inferred experimentally through measurements.

The heart of a Monte Carlo simulation is the pseudo-random number generator since its quality in terms of distribution and periodicity determines how well the random nature of the physical interactions is modelled. Pseudo-random number generation is an active area of research for which extensive reviews are available in the literature ${ }^{11-18}$. One important feature of the RNG is the periodicity. A RNG produces a precise sequence of pseudo-random numbers which depends on the initial seed of the sequence; a given seed always produces the same sequence hence the same simulation results. This is useful for debugging computer code simulations and for testing reproducibility of results.

In the field of radiotherapy physics and radiation dosimetry, Monte Carlo methods have been used for modelling electron and photon interactions with matter for the past 30 years and a good review of the techniques and methods is given by Jenkins et al. ${ }^{19}$. The characteristics of radiation beams used in radiation therapy and produced by medical linear accelerators, cobalt-60 machines, or brachytherapy systems, must be known very precisely. The treatment machine head and phantom or patient geometry have to be known precisely to follow properly the location of interactions and their results. Secondly, a precise knowledge of the probability distributions governing individual interactions of photons and electrons, described in Chapter 2 and Chapter 3, in various materials is required to simulate the random trajectories and energy depositions of individual particles. Each simulated particle 
produces one history; a collection of many millions of histories will yield knowledge on macroscopic measurable quantities, such as the absorbed dose in the patient or phantom, the relative contribution of the particle type to the dose, the relative contribution of the various components of the linear accelerator to the dose, and many other quantities, many of them actually impossible to measure physically. The statistical uncertainty of a simulation depends on $N$, the number of particle histories simulated, and generally decreases as $N^{-1 / 2}$.

A typical Monte Carlo software package for modelling radiation in medical physics will have the following components:

1. interaction cross-section data for photons and electrons/positrons

2. particle transport algorithms

3. geometry modelling

4. simulation data analysis

Many software packages are available for doing Monte Carlo simulations applied to medical radiation physics. Berger and Seltzer developed a "Class-I" Monte Carlo system, the ETRAN (Electron TRANsport) ${ }^{20}$ code from which various other codes, such as CYLTRAN, Integrated TIGER Series (ITS) ${ }^{21}$, and $\mathrm{MCNP}^{22}$, were developed. In a "Class I" MC system the particles move on a pre-determined energy loss grid. For our work, we used codes that are based on the EGS (Electron-Gamma-Shower) series ${ }^{23}$. We used the EGS4/PEGS4 ${ }^{24}$ MC code system along with the BEAM ${ }^{25}$ user code and the most recent EGSnrc code ${ }^{26-29}$. EGS4/EGSnrc-based MC systems are Class-II codes for which a threshold energy value is used to separate discrete or "catastrophic" interactions, which happen when the particle's energy is above the threshold, to continuous slowing down approximation (CSDA) model when the particle's energy is below the threshold.

\section{Egs Monte Carlo Code System}

EGS (Electron Gamma Shower) is a Monte Carlo computer code system which simulates the interaction of photons, electrons and positrons in matter. This code was originally developed at the Stanford-Linear Accelerator Centre (SLAC) by Ford and Nelson ${ }^{23}$. 
The EGS4 system, is a coupled photon-electron Monte Carlo code developed by W.R. Nelson, H. Hirayama, and D.W.O. Rogers ${ }^{24}$ with the PEGS4 (Preprocessor for EGS4) cross section preparation package. Those programs come with a MORTRAN3 pre-compiler which converts MORTRAN3 code into FORTRAN 77 code. EGS4 is programmed entirely in MORTRAN3 that was specifically created for that simulation package ${ }^{24}$.

\section{II.A. PEGS4}

One of the most important inputs for Monte Carlo simulations of medical radiation transport simulations is the interaction cross section data used in sampling the outcome of every individual particle step. All the cross-section data that EGS4 requires for the simulations is compiled by PEGS4, which generates cross section data for elements, compounds and mixtures from cross section tables of all elements with atomic numbers ranging from 1 to 100 . The basic tables are provided by Berger and Seltzer ${ }^{30}$ and are also the ones adopted by ICRU Report \# 37 (Ref. \#31). The cross sectional data for the material (medium) in question is calculated through a piece wise linear fit of the log of the cross section data over a large number of energy intervals (few $\mathrm{keV}$ to several thousand $\mathrm{GeV}$ ). The cross section files are specially formatted for direct use by EGS4. A given medium file prepared by PEGS4, provided its energy range is adequate, can be used by different simulations and only has to be generated once.

\section{II.B. EGS4/EGSnrc}

The EGS4/EGSnrc code is valid for particle energies ranging from about $1 \mathrm{keV}$ to several thousand $\mathrm{GeV}$ for photons, and from approximately $10 \mathrm{keV}$ to several thousand $\mathrm{GeV}$ for electrons and positrons. EGS4/EGSnrc has been tested extensively for the energy range of interest in medical physics. Complete bibliography of benchmarking and novel applications of the EGS system in the medical physics field is available on the web at the following URL address:

http://www.irs.inms.nrc.ca/inms/irs/papers/egs.biblio/egs.biblio.html 
When a particle is generated, it produces a cascade shower that includes the initial particle and all of its progeny as it travels through the medium. For a particular shower, many particle interaction processes will occur and the code will accurately simulate the physical situation if all of these processes are taken into account. For electron and positron transport, EGS considers the following: bremsstrahlung production, positron annihilation, Møller ( $\left.\mathrm{e}^{-}\right)$and Bhabha $\left(\mathrm{e}^{+}\right)$scattering as well as continuous energy loss between successive discrete interactions for electrons travelling through a medium. For photon transport Compton scattering, photo effect, pair production, and Rayleigh scattering are the processes taken into account.

EGS4 and EGSnrc use the "Lagged-Fibonacci Method" pseudo random number generator proposed by George Marsaglia and Arif Zaman ${ }^{12}$. EGSnrc also allows the use of "Luxury Pseudorandom Numbers" based on a "subtract-and-borrow" random number generator proposed by Marsaglia and Zaman, implemented by James ${ }^{18}$ and later improved by Luescher $^{13}$.

The particle transport in EGS4 is based on the mean free path $\lambda$, the average distance a particle will travel before suffering an interaction. The mean free path depends on the total molecular cross-section $\sigma_{t}$, which is proportional to the probability of interaction and is given by

$$
\lambda=M / N_{a} \rho \sigma_{t}
$$

where $M$ is the molecular mass, $N_{a}$ is Avogadro's number, and $\rho$ is the medium's physical density. The number of mean free paths $N_{\lambda}$ between two positions $x$ and $x_{o}$ is given by

$$
N_{\lambda}=\int_{\mathrm{x}_{0}}^{\mathrm{x}}[\lambda(x)]^{-1} \mathrm{dx}
$$

where $N_{\lambda}$ is sampled with the help of an RNG and is used to find the next location of interaction. The mean free path changes if either the particle enters a new medium or undergoes an interaction. At the next interaction location, the type of interaction process is determined randomly from weighted individual interaction cross-sections. The chosen interaction pro- 
cess, at the location in question, will determine parameters such as recoil and scatter directions and energy transfers.

For photon transport, the interaction cross-sections are sufficiently small that particle transport can be approximated by assuming that photons travel in a straight line with constant energy between interactions. EGS4/EGSnrc samples $N_{\lambda}$ to calculate $\lambda$ and to evaluate $N_{\lambda} \lambda$. When the particle enters a new medium, $\lambda$ is re-evaluated. $N_{\lambda}$ is decremented by the proper amount according to the number of $\lambda$ 's traversed during the transport step. The whole process is repeated until $N_{\lambda}=0$, which determines when the particle will interact.

For charged particles (electrons and positrons) the interaction cross section becomes very large as the energy of the particle approaches zero. For this reason, simulation at every interaction becomes impractical because hundreds of thousands of interactions would have to be modelled. EGS4/EGSnrc uses threshold energies, labelled AE for electrons and AP for photons, to solve this problem. The threshold energies include the rest mass of the given particle. As long as a bremsstrahlung process or a Møller interaction results in the creation of a secondary particle above the threshold, its interactions are treated as discrete whereas when it is lower than the threshold it is treated in a continuous manner by grouping many interactions together. This technique, called class II "condensed history" method $(\mathrm{CH})$, was first proposed by Berger ${ }^{32}$ and is justified, since a large number of small momentum transfers occur and therefore the deviation from the average is small. In this method, the cumulative effect of individual interactions is taken into account by sampling the change of the particle's energy and direction of motion at the end of the step from appropriate multiple scattering distributions. This, however, can introduce a step size-dependent artifact as is the case in EGS4. EGSnrc, however, is an updated version of EGS4 that allows for an artifact-free $\mathrm{CH}$ simulation of electron transport ${ }^{27-29}$. EGSnrc achieves this greater accuracy by offering improved algorithms to calculate multiple scattering parameters, electron step, energy loss and exact boundary crossing algorithm. Most of these corrections are included in a new version of PRESTA (Parameter Reduced Electron Step Algorithm) (PRESTA-II), the algorithm used for determining the parameters for electron transport. EGSnrc code also incorporates electron spin effects for electron elastic scattering which were shown to be non-negligible for simulations in which the contribution 
of backscatter radiation is important, including simple percent depth dose curve calculations $^{26}$.

EGS4 and EGSnrc have the same program flow chart and components, illustrated in Fig. 6-1. They are composed of an EGS core code layer that the user does not have to modify and a user code layer with which the user normally interacts. All input parameters of the simulation are entered in the MAIN subroutine and the user specifies how to handle the geometry of the system in the HOWFAR subroutine. All simulation outputs are obtained from the AUSGAB routine. Many thoroughly tested user codes such as DOSRZ, FLURZ, XYZDOS, etc., are available offering standard templates for simulations. The user who wishes to use them has to provide an input file that contains control values for the simulation specifying all input and output parameters for the simulation.

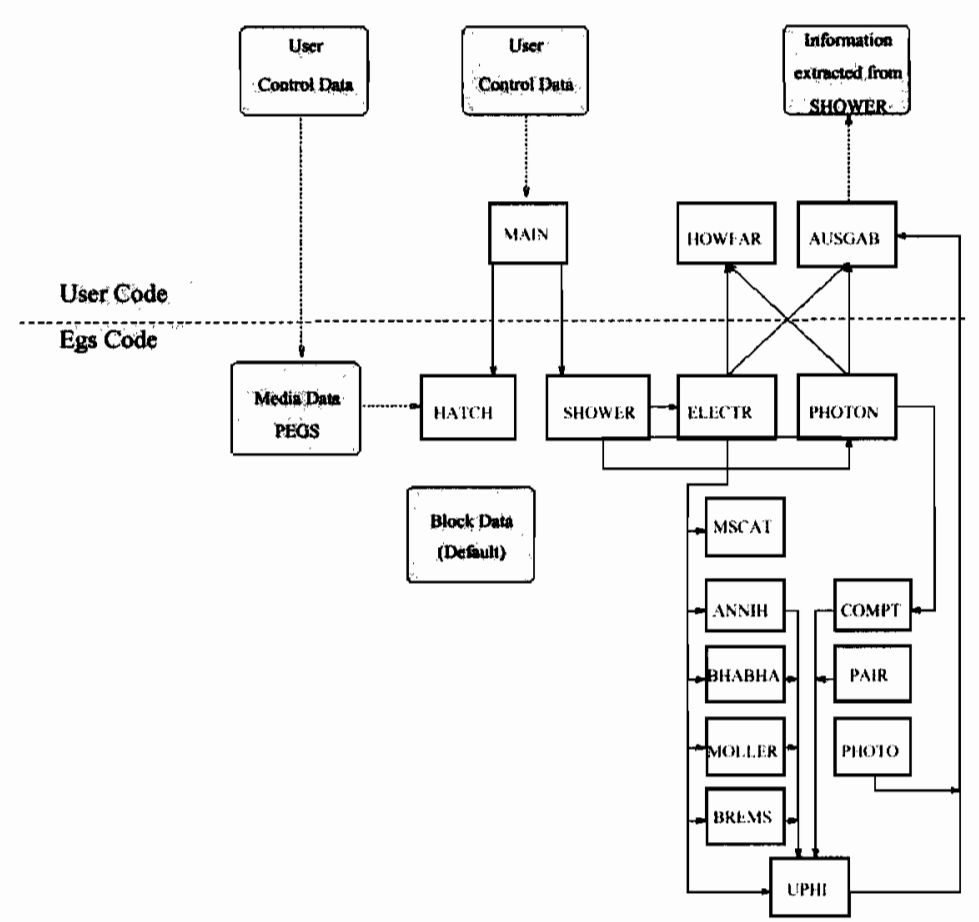

Figure 6-1. EGS program flow chart separating user code from core code (from Bielajew ${ }^{33}$ ). 


\section{II.B.1 EGS4/EGSnrc User Codes}

Many user codes are available for EGS4/EGSnrc. We mainly used three of them: DOSRZnrc and SPRRZnrc ${ }^{26,34}$ and DOSXYZ ${ }^{25}$. The codes simulate the passage of an electron or photon beam in a generalized geometry, either right cylindrical (RZ) or Cartesian (XYZ). DOSRZnrc and DOSXYZ are optimized to score absorbed dose in the defined geometry, while SPRRZnrc is used to calculate mean restricted stopping power ratios, $(\bar{L} / \rho)_{\text {med }_{2}}^{\text {med }}$, in the defined geometry.

The input parameters for execution of these programs are obtained from an input file in which the user specifies the geometry (size and material composition, etc.), the source type (energy, location, shape, etc.), and all of the Monte Carlo calculation parameters (number of histories, random number seeds, cut-off energies, variance reduction techniques to use, etc.) and finally the desired output quantities and formats.

\section{II.B.2 Variance reduction techniques}

The efficiency $\varepsilon$ of a Monte Carlo calculation is often formulated as:

$$
\varepsilon=\frac{1}{T s^{2}}
$$

where $T$ is a measure of the computing time and $s^{2}$ is the estimated variance associated with the calculated result. To increase efficiency, one has to reduce the variance for the same computing time. There is a trade-off between reducing the variance and computer time so that running more histories will reduce the variance but will require a longer computing time (unless the simulation is run on a faster computer). Often it is too costly, if not impossible, to run a sufficient number of histories to obtain the desired variance, and variance reduction techniques have to be used. Variance reduction techniques make calculations more efficient by reducing the time it takes to calculate a result with a given variance $s^{2}$. Some variance reduction techniques require various approximations and thus the gain in computing time may result in less accurate calculated results.

For electron transport simulation, many checks have to be made to verify whether or not the electron is crossing a region boundary. The number of checks can be 
reduced by keeping the total distance to the closest boundary when the electron enters a new region and at each step of transport the value is decreased by the step amount. This variance reduction technique speeds up calculations when electrons are transported in large regions and is included into the PRESTA algorithm of EGS4/EGSnrc. PRESTA saves computing time by employing small steps only in the vicinity of the boundaries and large electron steps when the electron is far from boundaries. When an electron cannot reach a boundary of another geometrical element from its actual location, the history of this electron can be terminated on the spot and its energy deposited locally. This technique is called range rejection. One error introduced by this technique is that subsequent bremsstrahlung photons emitted from that electron that may have escaped the actual geometrical region are lost.

One of the main problems for photon transport is that for a given region of interest efficiency is lost because many of the photons will travel across that region without interacting. A solution to this problem is to use a technique called photon forcing. This technique forces photons to interact and reduce their statistical weight for the calculation using a probability distribution based on the total number of mean free paths along the direction of motion of the photon to the end of the geometry.

Russian roulette and particle splitting are used when the region of interest of a given application comprises only a fraction of the geometry of the simulation. One photon can be "split" into many photons as it approaches a region of interest, these photons will later play "Russian roulette" as they leave that region to determine which one will survive, again with appropriate particle weight carried along.

Secondary particle enhancement is another similar variance reduction technique. Instead of creating one secondary particle, $N$ of them are created with a statistical weight of $1 / N$ and each of them will have a different energy and creation angle sampled from a known distribution. This method is more sophisticated than the splitting method where $N$ identical particles are produced. However, energy conservation is violated for the incident particle history but, over many interactions, energy conservation is preserved on average since Russian roulette is played. A typical application of that method is bremsstrahlung splitting which is available in the EGS4/EGSnrc codes. 


\section{BeAm Monte Carlo Code System}

BEAM is a Monte Carlo simulation system ${ }^{25}$ for modelling standard radiotherapy units including high-energy electron and photon beams, cobalt-60 beams and orthovoltage units. The BEAM computer code incorporates the EGS4 Monte Carlo computer code and is basically a front-end to the EGS4 code system. It presents an easy user interface to input the geometry, the physical parameters of the simulation and the means of analyzing and outputting the results.

The use of the BEAM code simplifies greatly the modelling of treatment machines by predefining a large variety of elementary geometric structures, called component modules (CM). The beam scripts automatically create an EGS4 user code for a given treatment machine based on the geometry entered by the user using the $\mathrm{CM}$ as building blocks. The CM are specifically coded so as to mimic various components of treatment machines such as jaws, applicators, cones, mirrors, ion chambers, etc. The code uses many variance reduction techniques such as range rejection, Russian roulette, bremsstrahlung splitting and photon forcing. It can analyze and output many different quantities and information calculated by the simulations. It has been extensively tested over the past years and a complete list of publications is available online at the following URL:

http://www.irs.inms.nrc.ca/inms/irs/BEAM/bibliog/omega_pubs.html

Simulations of treatment machines are normally performed to produce a phase-space file which contains the list of all particle characteristics which have reached a particular plane (at a particular $z$ position). The stored characteristics of a particle are the type of particle, its energy, its location on the phase-space plane $(x, y)$, its velocity vector $\left(v_{x} v_{y}, v_{z}\right)$ and the latch. The latch is an 8 bit variable which is a powerful feature of the BEAM system. It allows storing the number of the last region of interaction of the particles or flags for regions of interest where the particle has interacted. Using the latch one can determine or differentiate the contribution to the phase-space or to an anteriorly calculated dose from particles created or scattered from various regions of the CMs of the accelerator. 
The creation of phase-space is a very useful feature, since a given phase-space can be "reused" as a particle source input in further simulations, either for another BEAM code or with EGSnrc or EGS4 user code. Typically a phase-space which is statistically representative of a treatment machine beam contains many millions of particles and will take many megabytes of disk space.

When using the phase-space as a source input for other simulations, the phase-space particles can be reused without any loss of accuracy since each history will have a different random number seed. For beams which present a symmetry, for example, an open "non-modulated" rectangular field, all created particles can be re-localized by symmetry into the 3 other quarters of the field. The beam package includes a program called beamdp that can analyze the content of a phase-space file. It can calculate from the phase-space various parameters, such as energy fluence, particle fluence, spectral distribution, mean energy, angular distribution and weight distribution. A flow chart of the BEAM/EGS4 and EGSnrc to model a radiotherapy treatment machine is given in Fig. 6-2.

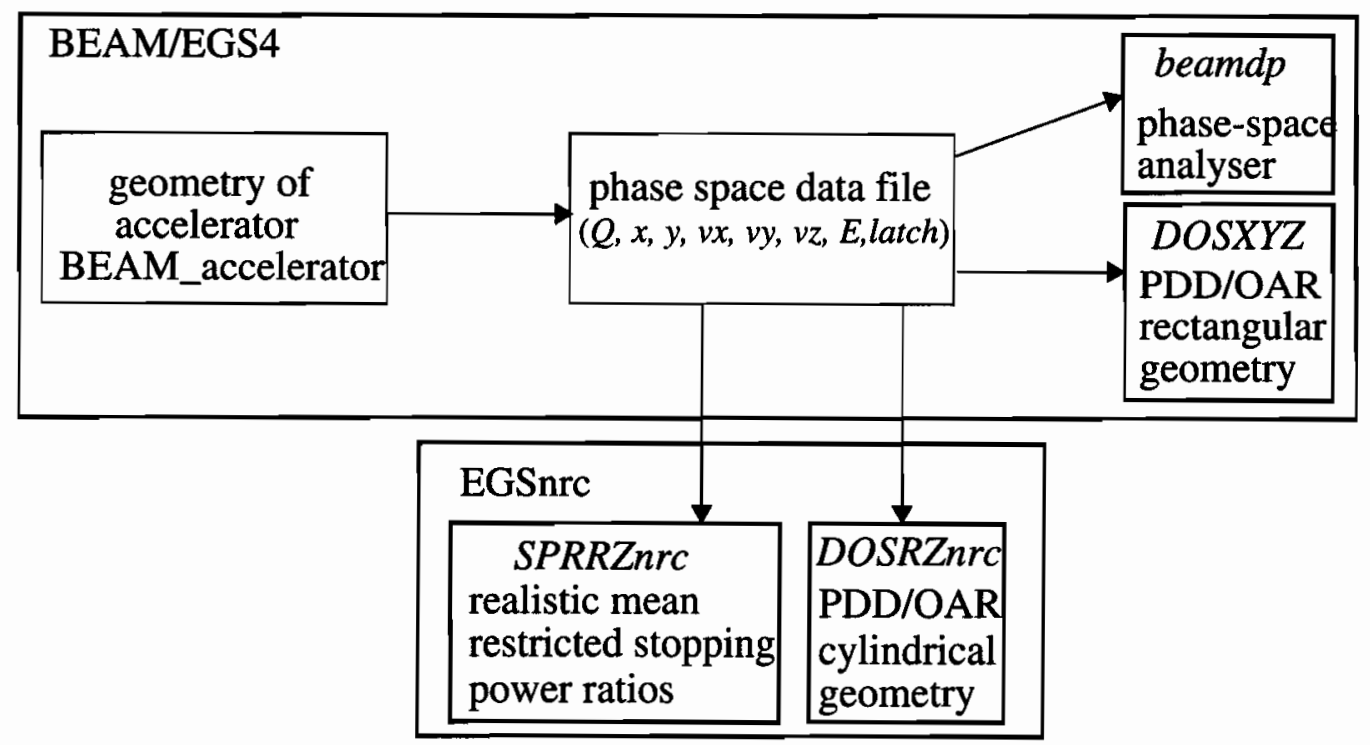

Figure 6-2. Flow chart of simulation process for a radiotherapy treatment machine. 


\section{Monte Carlo: Montreal General Hospital THERAPY MACHINES}

Some of the radiotherapy units available at the Montreal General Hospital were modelled using the BEAM Monte Carlo code system. First, the exact dimensions and compositions of the radiotherapy machine treatment heads were carefully entered in the BEAM input files using appropriate component modules to match the geometry. Three different types of machines were modelled: an isotope machine (cobalt-60), a medical linear accelerator in an electron mode (Varian Clinac-18), and a medical linear accelerator in a photon mode (Varian Clinac-2300 C/D). The isotope machine was modelled from first principles by generating the photons from sampling the exact ${ }^{60} \mathrm{Co}$ spectrum source and considering the exact source and source enclosure dimensions. For a medical linear accelerator, the Monte Carlo simulations typically begins at the exit window of the accelerator where a narrow circular beam of previously accelerated electrons with energy $E_{o}$ is taken for the source of the simulations. Extensive discussions of medical linear accelerator principles are available in the literature ${ }^{35,36}$. Typical treatment head geometries and designs are illustrated in Fig. 6-3 for the photon and electron treatment modes. In the photon mode, photons are produced by bremsstrahlung emission in the x-ray target. The forward peaked x-ray beam produced is later filtered by the flattening filter in order to achieve a flat dose distribution at depth in the patient or phantom. In the electron mode, there is no $\mathrm{x}$-ray target and the electron beam is scattered by a thin metallic foil (scattering foil) to produce a flat beam at the position of the patient. Applicators or electron cones are used to collimate the highly scattered electrons in order to produce a practical collimated clinical beam.

All EGS4/EGSnrc Monte Carlo calculations were performed using PEGS4 data

files that were created with ICRU-37 data and density corrections ${ }^{31}$. Cut-off values of $10 \mathrm{keV}$ for electrons (ECUT) and photons (PCUT) were used for BEAM, DOSXYZ, and DOSRZnrc calculations. 
(A)

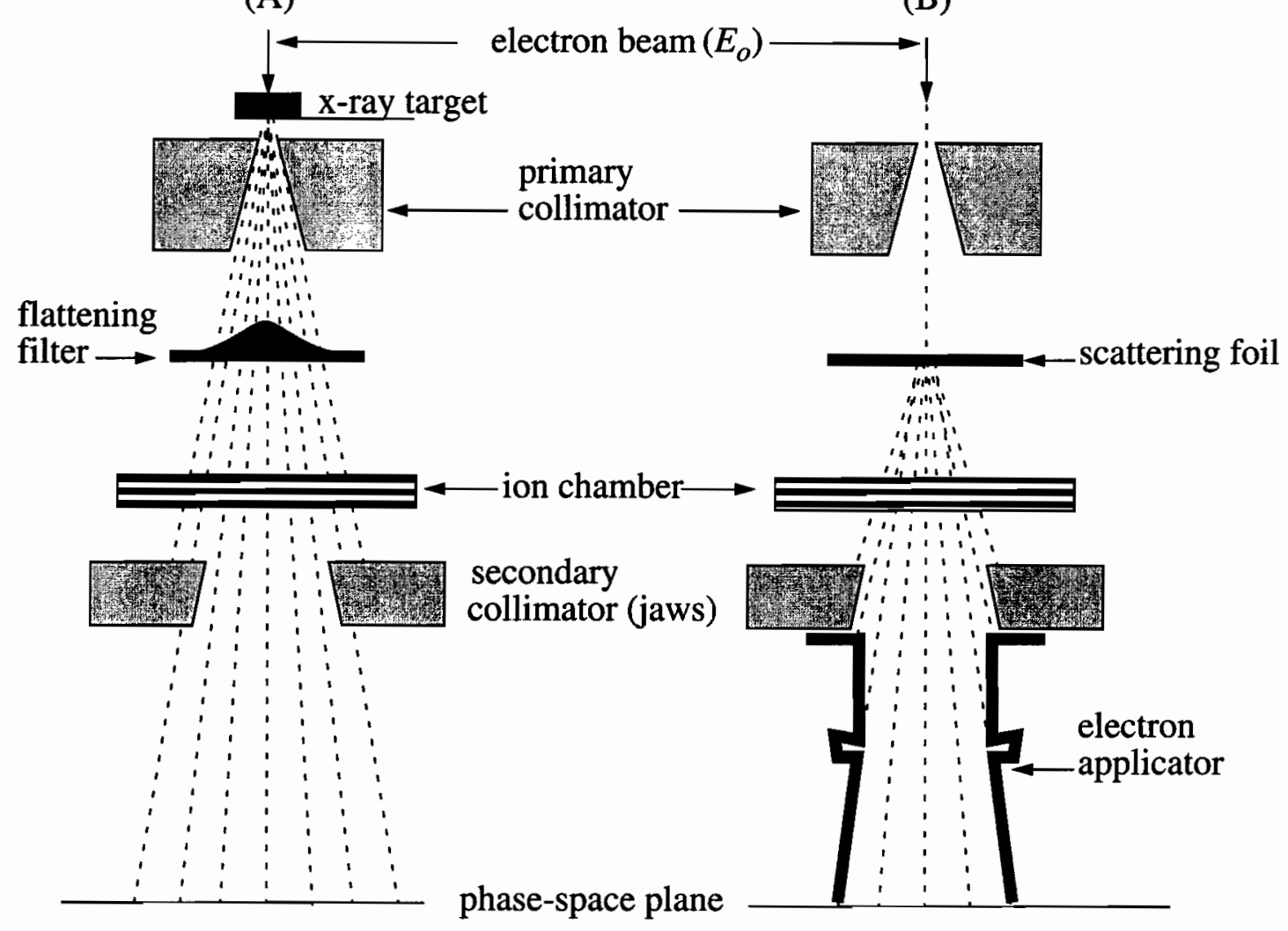

Figure 6-3. Treatment heads of medical linear accelerators in photon mode (A) and in electron mode (B). In photon mode, an $\mathrm{x}$-ray target and a flattening filter are used and in the electron mode a scattering foil and an electron applicator are required.

\section{IV.A. Varian Clinac-18}

When simulating a linear accelerator with the BEAM code, not only the geometry of the given medical linear accelerator has to be known, but also the initial kinetic energy of the electrons as they enter the treatment head $E_{o}$ must be optimized for the calculations to match the particular linac beam output. The standard procedure used to validate a beam simulation is to produce percent depth dose curves with EGSnrc and to compare them with measured data. In general, the initial kinetic energy $E_{o}$ of the electron beam is adjusted for the simulated $R_{50}$ value of the PDD curve (see Fig. 1-3) to match the measured 
one. This procedure is based on trial and error, and can be guided by a generalized relationship between $R_{50}$ and $E_{o}$ in water that was originally given by $\operatorname{Ding}^{37}$ and revised by Rogers $^{38}$ using EGSnrc which now includes the effect of electron spin on elastic multiple scattering. A given initial electron energy $E_{o}$ is specified, the simulation is performed and the calculated $R_{50}$ value is compared to the measured one and $E_{o}$ is adjusted accordingly. The process is repeated until good agreement is reached. The calculated beam profiles (off-axis ratios) can also be compared to measurements to ensure that the geometry is properly modelled.

The initial electron energies for the simulation of the $9 \mathrm{MeV}$ and $15 \mathrm{MeV}$ electron beam of the Montreal General Hospital's Varian Clinac-18, are 9.4 and $14.9 \mathrm{MeV}$, respectively. The initial electron beam is assumed to be a monoenergetic pencil beam of $0.1 \mathrm{~cm}$ radius. The $R_{50}$ values for the $10 \times 10 \mathrm{~cm}^{2}$ beams (applicator $10 \times 10 \mathrm{~cm}^{2}$ and jaws at $15 \times 15 \mathrm{~cm}^{2}$ ) in water for 9.4 and $14.9 \mathrm{MeV}$ are 3.45 and $5.72 \mathrm{~cm}$, respectively. Figure 6-4 shows a 3-D representation of the Clinac-18 in the electron mode, where the scattering foils and the electron applicators are seen. Some particle histories are also shown as lines.
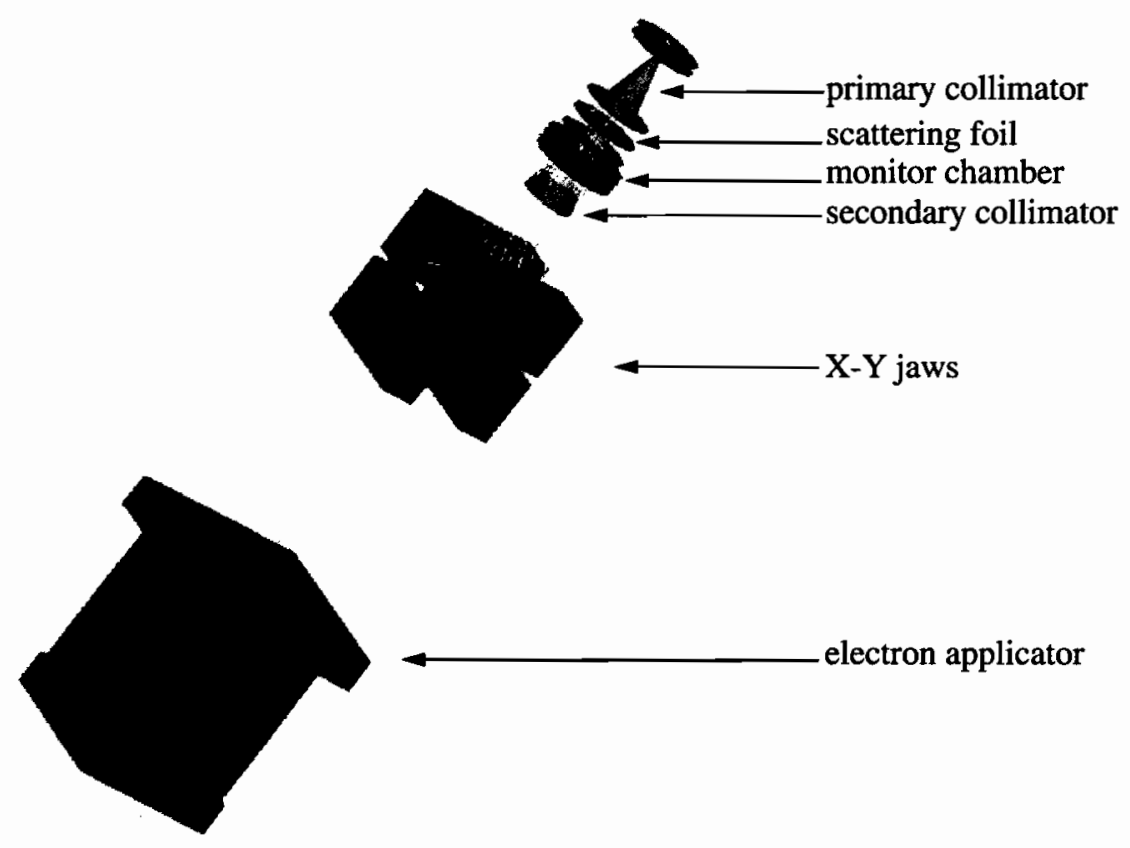

Figure 6-4. 3-D view of the Clinac 18 treatment machine head in electron mode 
Phase-space files for the two electron beams were obtained at SSD $=100 \mathrm{~cm}$ for both 9 and $15 \mathrm{MeV}$ electron beams. About 4000000 particles were accumulated into the phase-space files. Phase-space files for $15 \times 15 \mathrm{~cm}^{2}$ open fields, without electron applicator, were also generated for both electron energies. Figure 6-5 shows PDDs curves calculated with DOSRZnrc and measured with a Roos chamber (PTW-34001 Roos Chamber; PTW, Freiburg Germany) used in a 3-D RFA water tank (300 scanning water tank; Scanditronix, Uppsala, Sweden) for the $9 \mathrm{MeV}$ and $15 \mathrm{MeV} 10 \times 10 \mathrm{~cm}^{2}$ beams. Spin effect was turned on into the DOSRZnrc code and PRESTA-II was used for electron transport. Measurements were corrected for the variation of stopping power with depth. The relative error on measured and calculated values is less than $0.2 \%$ and both curves are identical within errors.

(A)

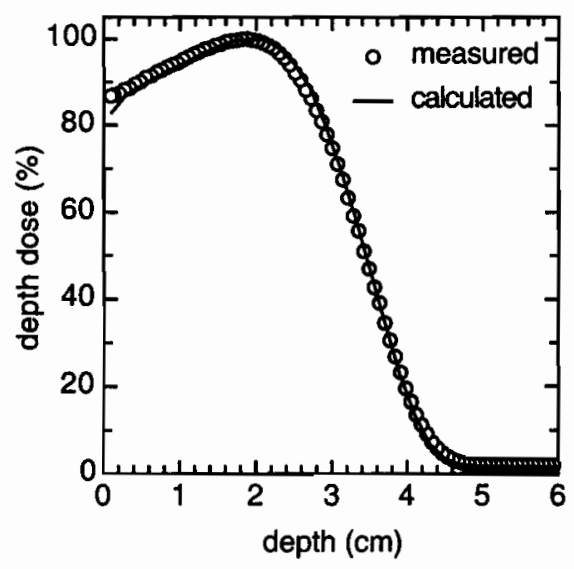

(B)

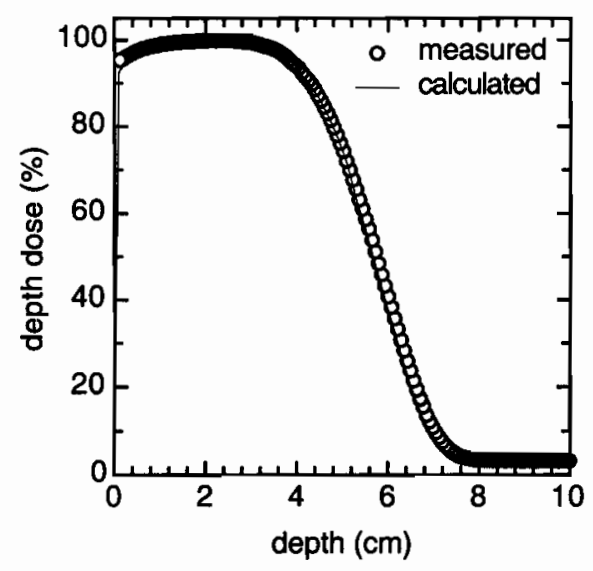

Figure 6-5. Percent depth doses measured in water with RFA and Roos chamber (circle dots) and calculated with DOSRZnrc (lines) for $9 \mathrm{MeV}$ (A) and for $15 \mathrm{MeV}$ (B) beams with $10 \times 10 \mathrm{~cm}^{2}$ applicators.

Figure 6-6 shows beam profiles at various depths measured with the RFA water tank with electron diodes (model F1421; Scanditronix, Uppsala, Sweden) and calculated (with DOSXYZ) for the $9 \mathrm{MeV} 10 \times 10 \mathrm{~cm}^{2}$ electron applicator. Data is normalized to a $100 \%$ at $d_{\max }$. The agreement between calculated and measured points is very good (less than $0.5 \%$ difference) which indicates that the geometry was properly modelled.

Figure 6-7 and Figure 6-8 show the percent depth doses measured with TLD techniques using $0.15 \mathrm{~mm}$ thick LiF chips (TLD-700; Harshaw Chemical Company, Solon, $\mathrm{OH})$ and calculated with DOSRZnrc in various materials for both 9 and $15 \mathrm{MeV} 15 \times 15 \mathrm{~cm}^{2}$ 
beams without an electron applicator. Data is normalized to a $100 \%$ at $d_{\max }$ for Solid Water ${ }^{\mathrm{TM}}$ (black square point). The calculated depth doses differ by less than $1 \%$ for aluminum, copper and bone material and by less than $2 \%$ for lung-equivalent material. The composition of the materials is given in Chapter 5, Table 1. These graphs illustrate and validate the possibilities of calculating the dose in various materials, which is important for PEEC measurement verifications. These data were used to obtain electron fluence ratios for various materials of clinical interest, relative to Solid Water ${ }^{\mathrm{TM}}{ }^{39}$.

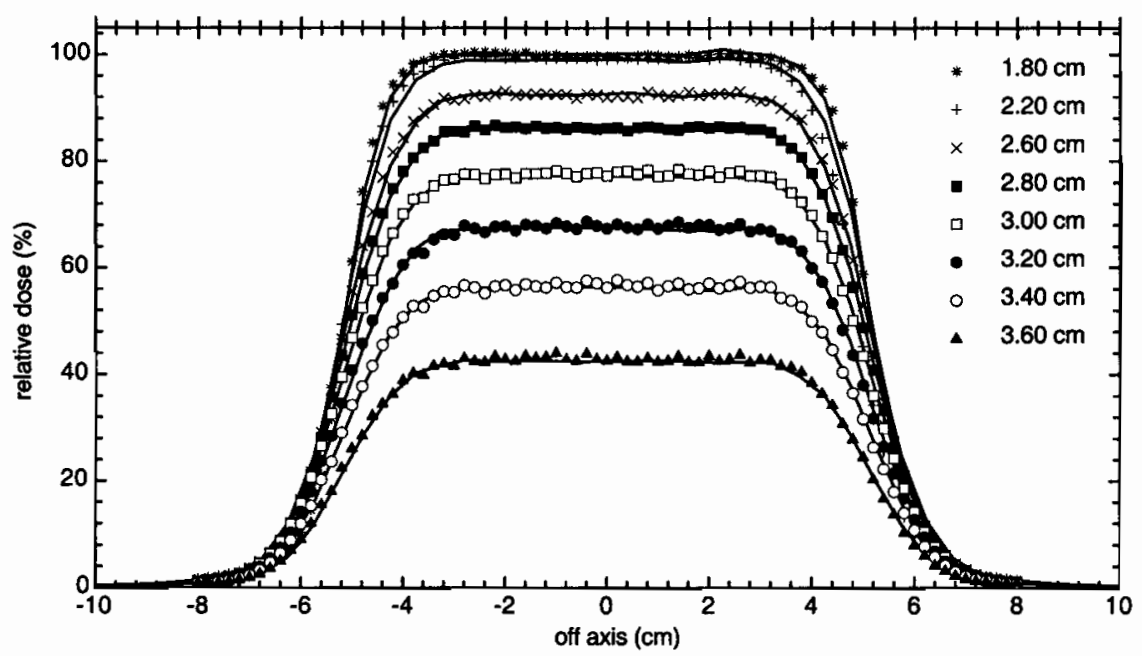

Figure 6-6. Profiles measured with RFA and electron diodes (dots) and calculated with DOSXYZ (lines) in water for $9 \mathrm{MeV}$ beam with $10 \times 10 \mathrm{~cm}^{2}$ applicator.
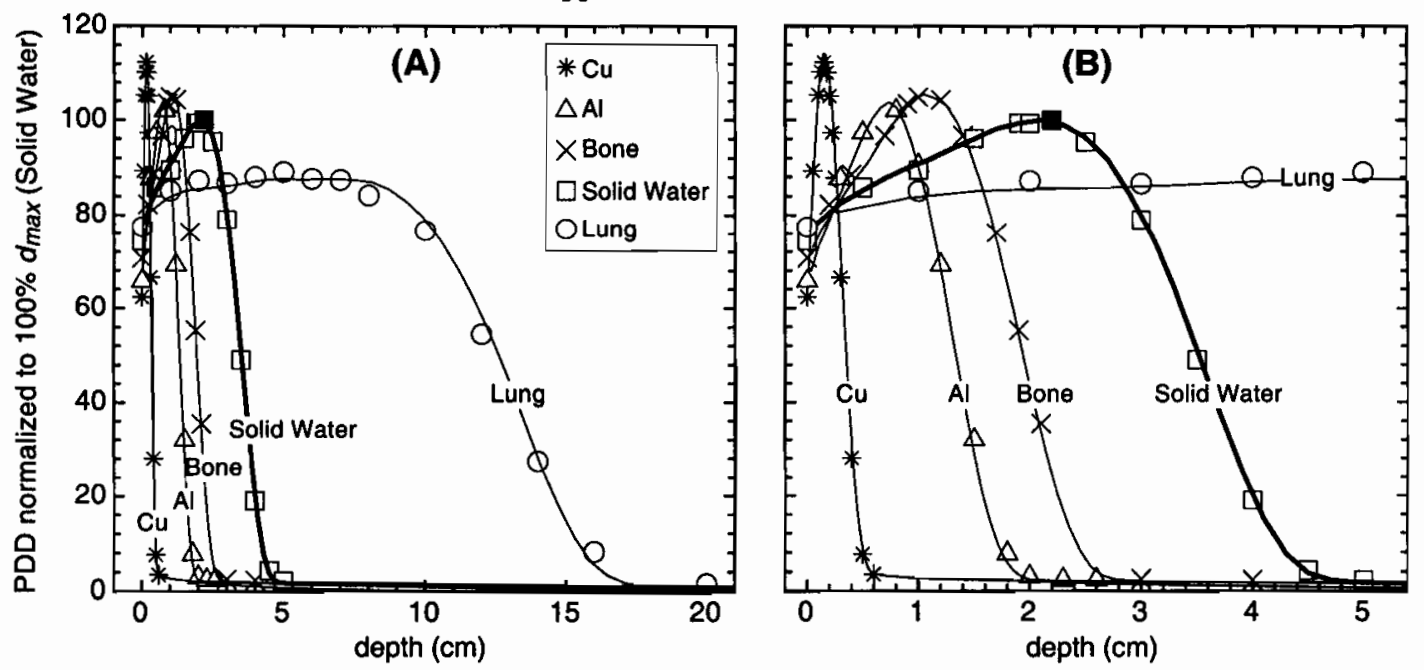

Figure 6-7. Percent depth doses measured with TLDs (dots) and calculated with DOSRZnrc (lines) in various materials for a $9 \mathrm{MeV} 15 \times 15 \mathrm{~cm}^{2}$ beam without electron applicator. Values are normalized to $100 \%$ to $d_{\max }$ in Solid Water ${ }^{\mathrm{TM}}$ (black square points). 

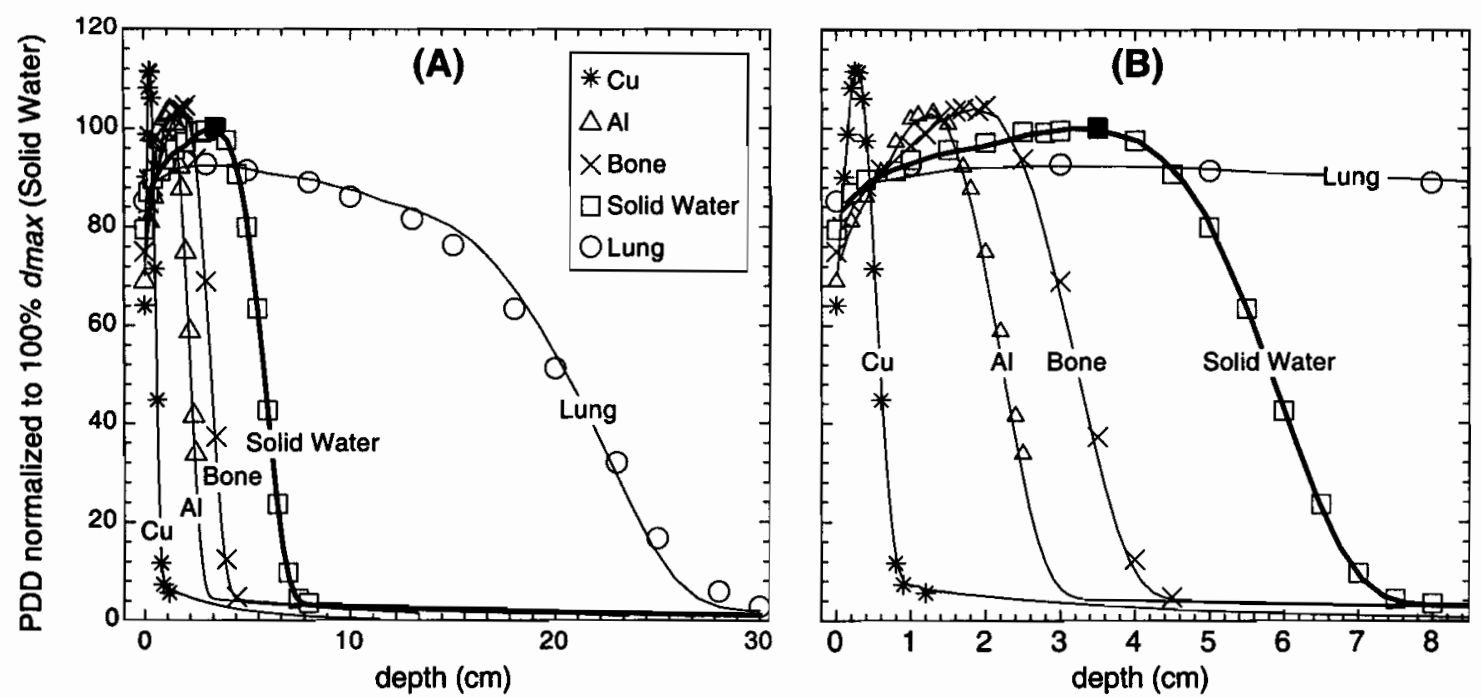

Figure 6-8. Percent depth doses measured with TLDs (dots) and calculated with DOSRZnrc (lines) curves in various materials for a $15 \mathrm{MeV} 15 \times 15 \mathrm{~cm}^{2}$ beam without electron applicator. Values are normalized to $100 \%$ to $d_{\max }$ in Solid Water ${ }^{\mathrm{TM}}$ (black square points).

\section{IV.B. Clinac-2300 C/D}

The 6 and $18 \mathrm{MV}$ photon beam treatment head geometries of the Montreal General's Varian Clinac-2300 C/D were also modelled with the BEAM code system. It was found that the optimized energies of the initial electron beams are respectively 6 and 17.8 $\mathrm{MeV}$. The initial electron beams were assumed to be monoenergetic electron pencil beams of $0.1 \mathrm{~cm}$ radius striking the x-ray target. A 3-D view of the machine is shown in Fig. 6-9(A) with the position of the phase-space relatively to the source at SSD $=100 \mathrm{~cm}$. Figure 6-9(B) shows a zoomed view of the components with some particle histories represented by lines. 


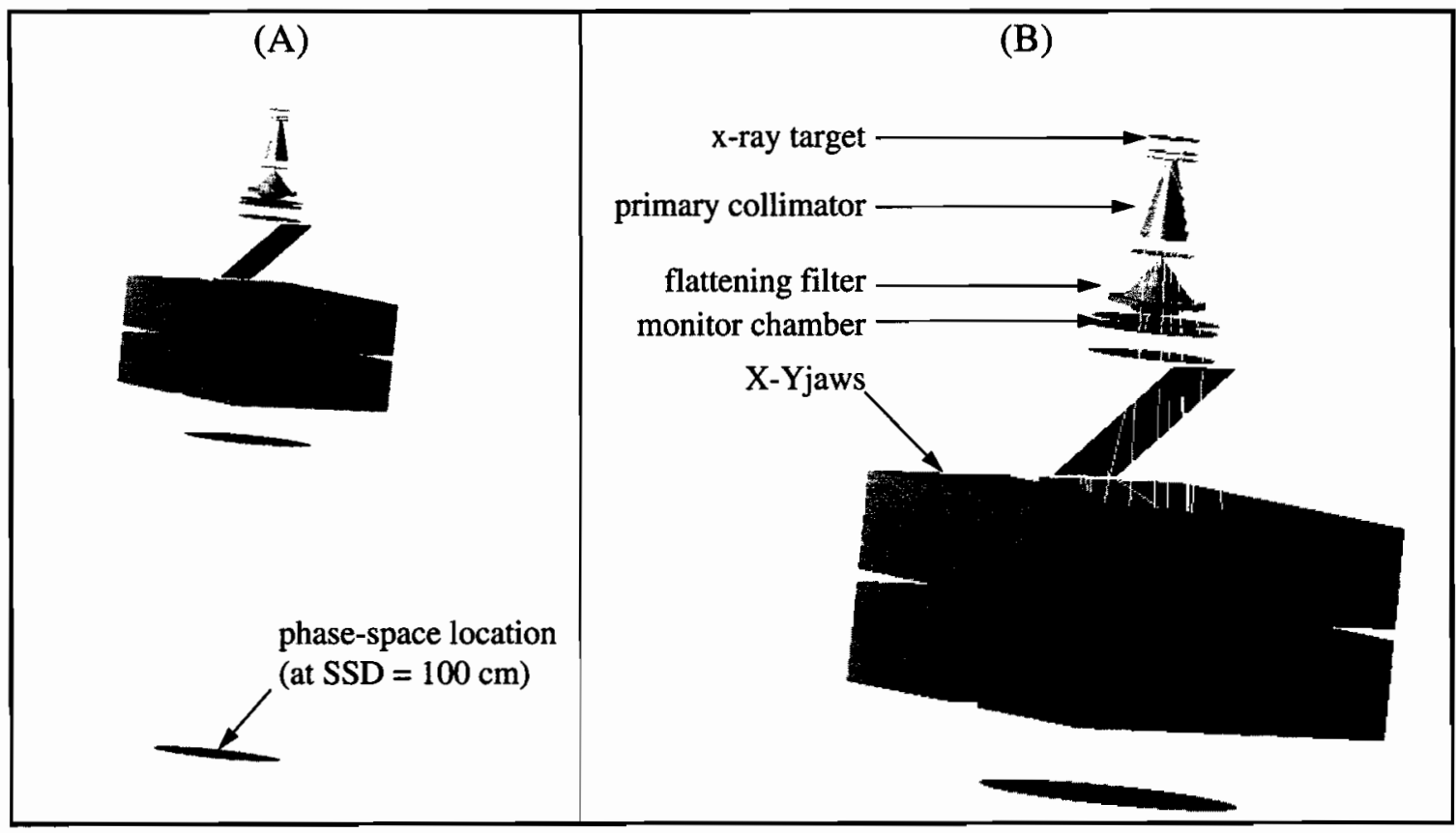

Figure 6-9. 3-D representation of the Clinac $2300 \mathrm{C} / \mathrm{D}$ treatment head components in photon mode. The location of the phase-space is shown in (A) and a zoom view on the component is shown in (B) with some particles histories represented by lines.

Phase-spaces for $10 \times 10 \mathrm{~cm}^{2}$ fields were obtained at $\mathrm{SSD}=100 \mathrm{~cm}$ using bremsstrahlung splitting variance reduction technique (factor of 20) for both the 6 and $18 \mathrm{MV}$ photon beams. About 4 million particles were accumulated into each phase-space. Figure 6-10 shows percent depth doses calculated with DOSRZnrc and measured with the PEEC for both Solid Water ${ }^{\mathrm{TM}}$ and bone-equivalent material. Spin effect was turned on for DOSRZnrc simulations and PRESTA-II was used for electron transport. The relative error on both measured and calculated values is less than $0.2 \%$ and both curves are identical within their error.

Calculated (DOSXYZ) and measured (RFA tank and ion chambers (RK ion chambers; Scanditronix, Uppsala, Sweden)) off-axis beam profiles are shown in Fig. 6-11 and Fig. 6-12 for both 6 and $18 \mathrm{MV} 10 \times 10 \mathrm{~cm}^{2}$ beams, respectively. All curves are normalized to $100 \%$ at $d_{\text {max }}$. The relative error on both measured and calculated values is less than 
$0.5 \%$ for the whole range of profiles and the difference between the measured and calculated values is less than $1 \%$ except for the penumbra region where a larger difference is observed. Penumbra is not of importance in this work, since we are mainly concerned with dose calculation along the central axis of single beams. The problem is caused by the difficulty of measuring the penumbra because of the variation of the ion chamber response. The measurement of penumbra is relevant only in some specialized treatment techniques in radiation therapy, such as intensity modulated radiation therapy (IMRT) and stereostactic radiosurgery. Therefore verification of IMRT fields with Monte Carlo simulations is important, if no accurate measurement techniques are available.
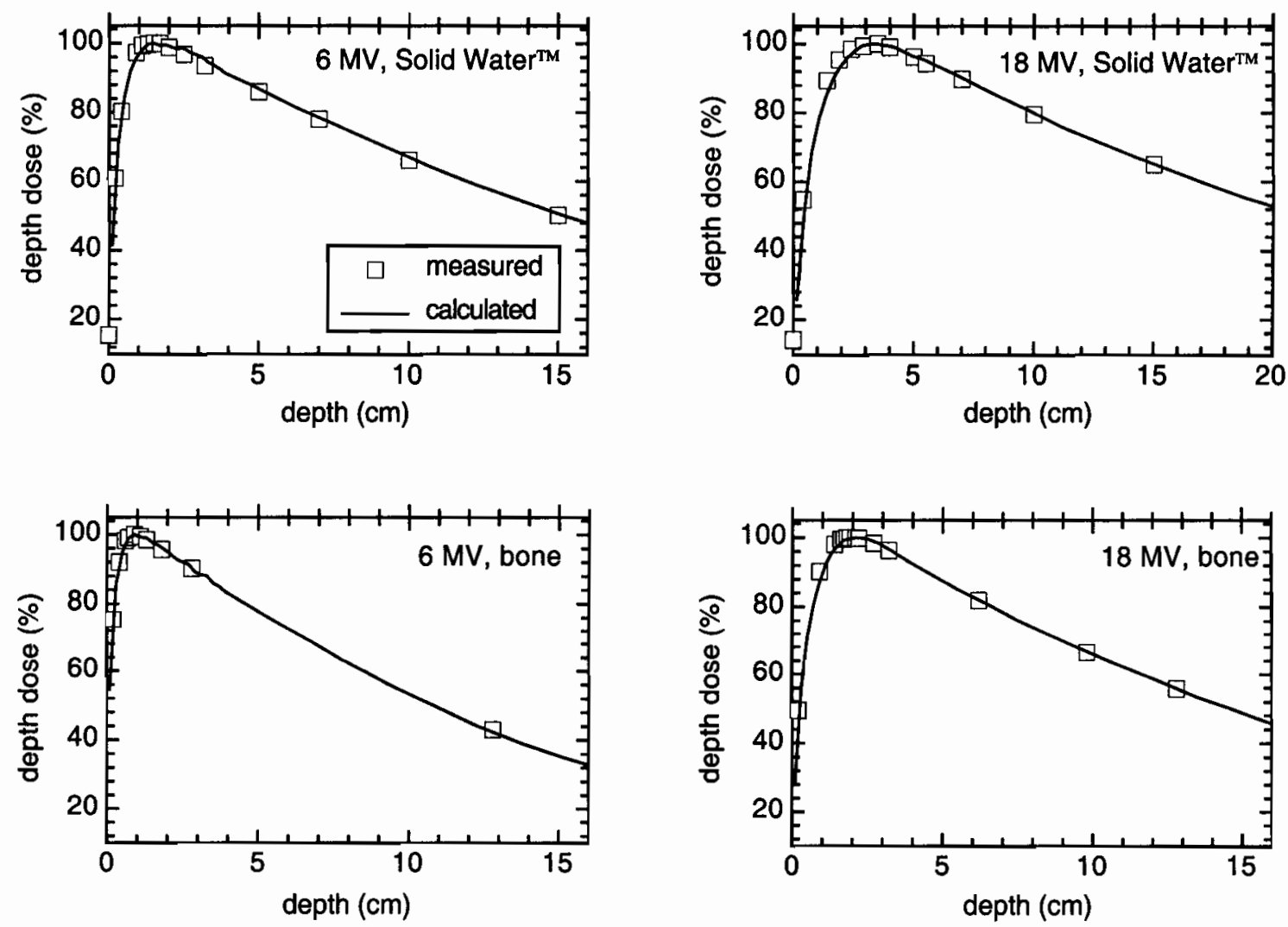

Figure 6-10. Percent depth dose curves for 6 and $18 \mathrm{MV} 10 \times 10 \mathrm{~cm}^{2}$ fields, SSD $=100 \mathrm{~cm}$, in Solid Water ${ }^{\mathrm{TM}}$ and bone-equivalent material, measured with PEEC (square dots) and calculated with DOSRZnrc (lines). 


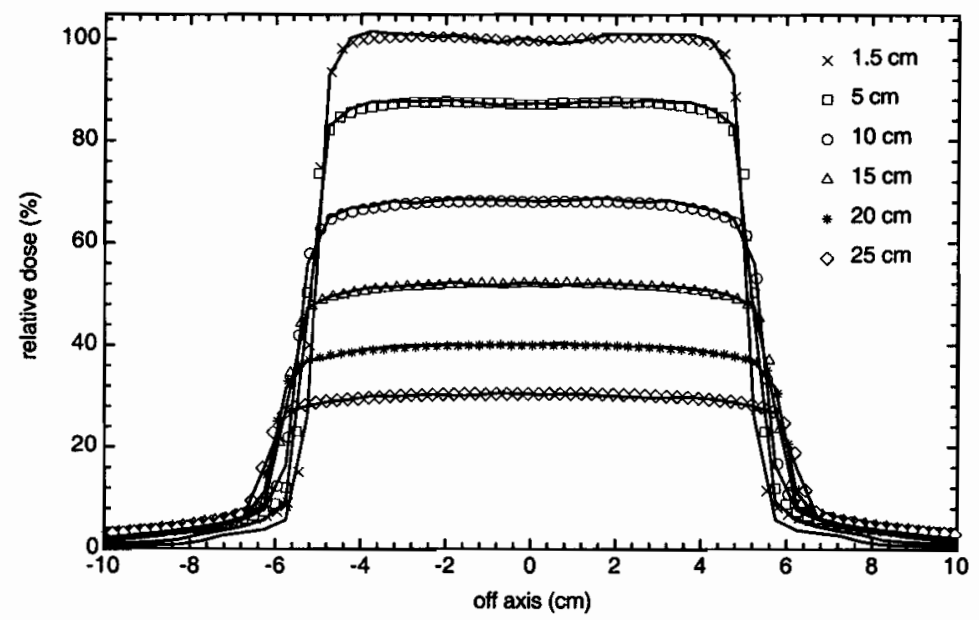

Figure 6-11. Off-axis profiles for the Clinac-2300 C/D $6 \mathrm{MV}$ beam $10 \times 10 \mathrm{~cm}^{2}$ measured with RFA and RK chambers and calculated with DOSXYZ (lines) in water.

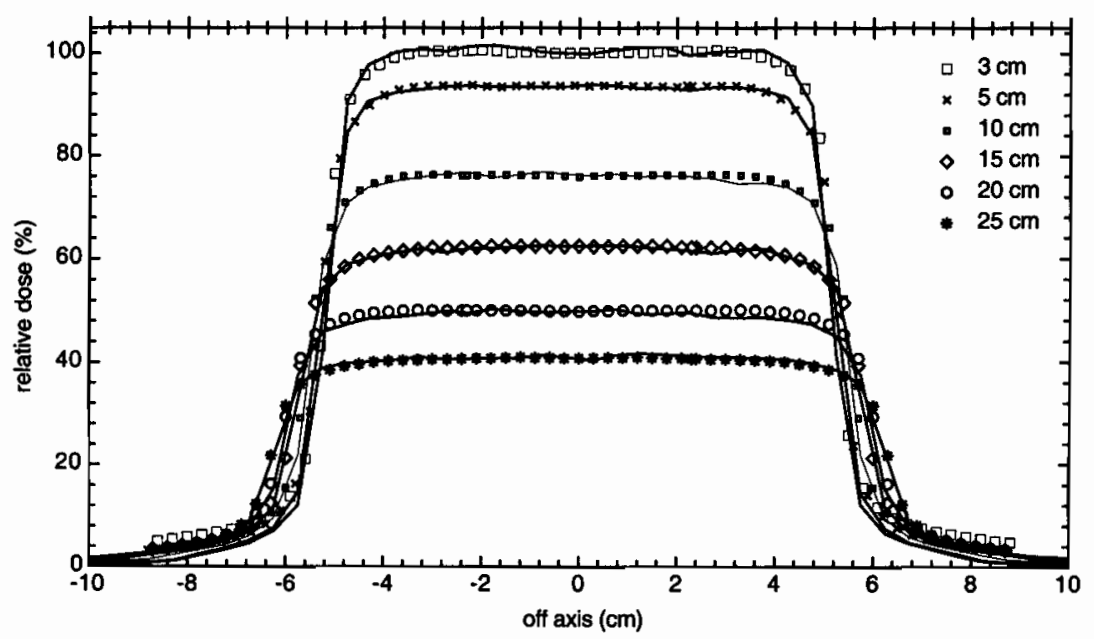

Figure 6-12. Off-axis profiles for the Clinac-2300 C/D $18 \mathrm{MV}$ beam $10 \times 10 \mathrm{~cm}^{2}$ measured with RFA and RK ion chambers (dots) and calculated with DOSXYZ (lines) in water.

\section{IV.C. Theratron T-780 (Cobalt-60 machine)}

The precise geometry of the T-780 cobalt-60 machine was used to create the beam model of the treatment machine. The exact dimensions and shape of the source were used to generate photons according to the ${ }^{60} \mathrm{Co}$ energy spectrum $(1.175 \mathrm{MeV}$ (99.88) and 1.335 MeV (100)). Figure 6-13 shows a side view and a 3-D representation of the geometry 
of the treatment head of the machine. The source is encapsulated into a lead housing and the beam is collimated by a series of lead and depleted uranium collimators. Figure 6-14 shows percent depth doses for Solid Water ${ }^{\mathrm{TM}}$ and bone-equivalent material, calculated with DOSRZnrc and measured with PEEC for a $10 \times 10 \mathrm{~cm}^{2}$ field at source to surface distance (SSD) of $100 \mathrm{~cm}$. The agreement between measured and calculated data is very good (within $1 \%$ ) and the relative error on both calculated and measured data is less than $0.5 \%$.

(A)

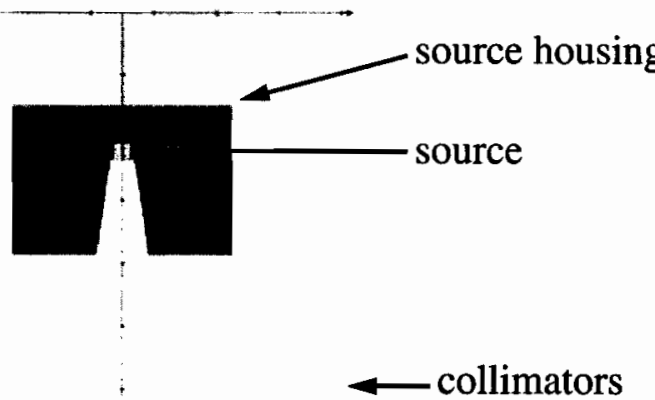

(B)

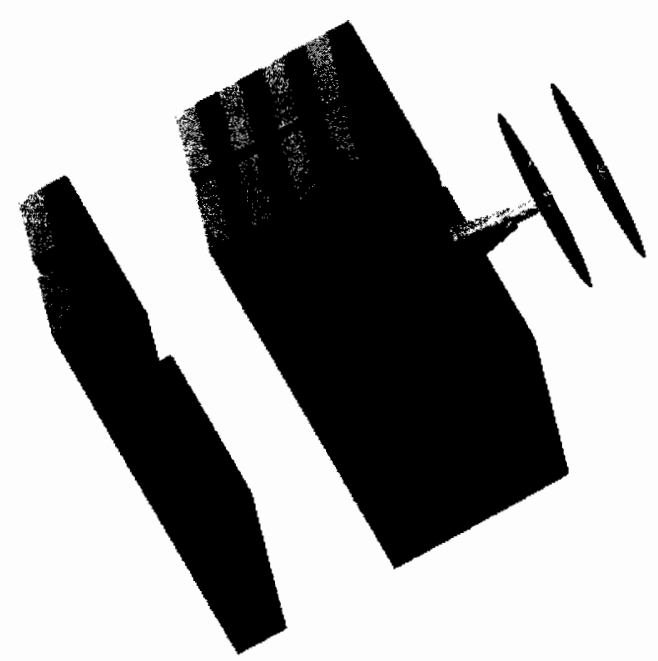

Figure 6-13. A side view (A) and a 3-D (B) representation of the T-780 treatment machine head.

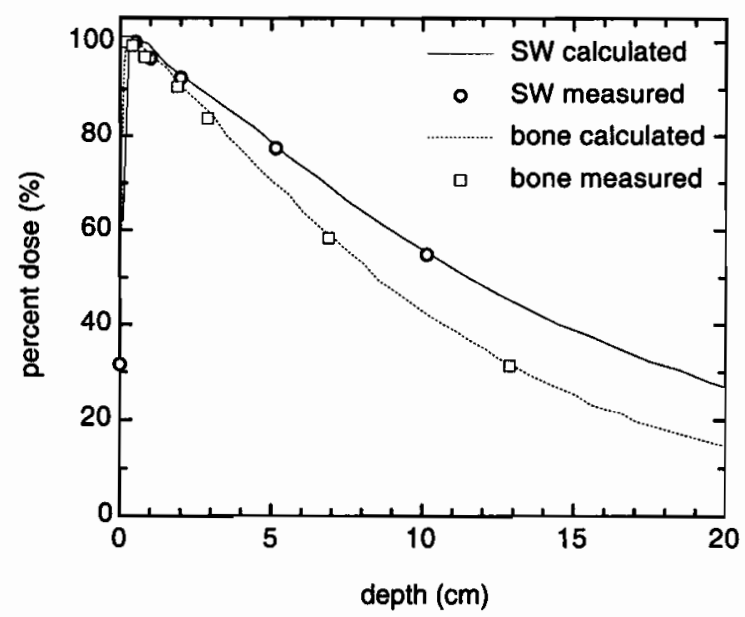

Figure 6-14. Percent depth dose curves for Solid Water ${ }^{\mathrm{TM}}$ and bone-equivalent material measured with PEEC (circles and squares) and calculated with Monte Carlo (lines). 


\section{IV.D. Clinical beam energy spectra}

For each clinical beam we calculated a phase-space file, which contains the important physical characteristics of millions of particles reaching the phase-space plane. The beam energy spectrum data can then be calculated from the phase-space file. We calculated beam energy spectra for the clinical beams used in our experiments. The spectra are shown in Fig. 6-15, Fig. 6-16, Fig. 6-17, Fig. 6-18, Fig. 6-19 for the Clinac-18, 9 and $15 \mathrm{MeV}$ electron beams, for the Clinac-2300 C/D, 6 and $18 \mathrm{MV}$ photon beams and for the T-780 cobalt -60 photon beam, respectively. For the electron beams we also show the associated photon contamination spectra since a typical clinical electron beam is also composed of a large number of low energy photons.
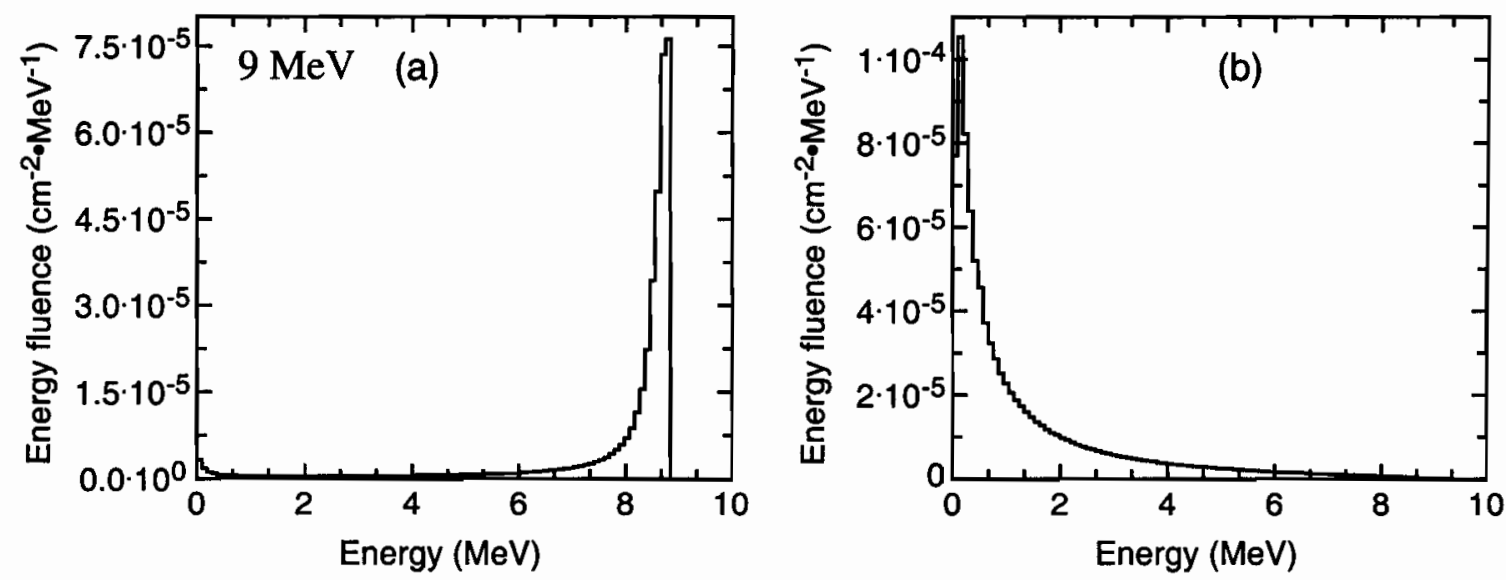

Figure 6-15. (a) Electron spectrum (Clinac-18) for a $9 \mathrm{MeV}$ electron beam (SSD $=100 \mathrm{~cm}$, field size $=10 \times 10 \mathrm{~cm}^{2}$ ) with (b) associated photon contamination spectrum. The spectra were calculated with Monte Carlo technique. 

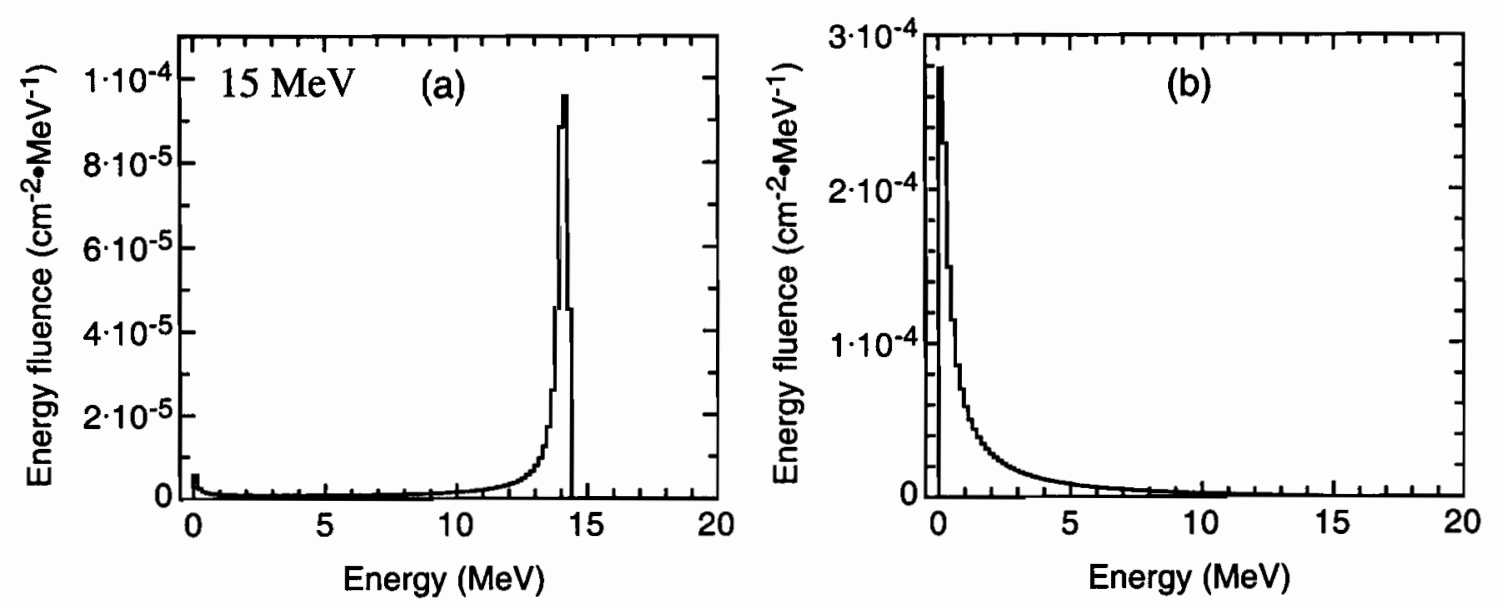

Figure 6-16. (a) Electron spectrum (Clinac-18) for a $15 \mathrm{MeV}$ electron beam ( $\mathrm{SSD}=100 \mathrm{~cm}$, field size $=10 \times 10 \mathrm{~cm}^{2}$ ) with (b) associated photon contamination spectrum. The spectra were calculated with Monte Carlo technique.

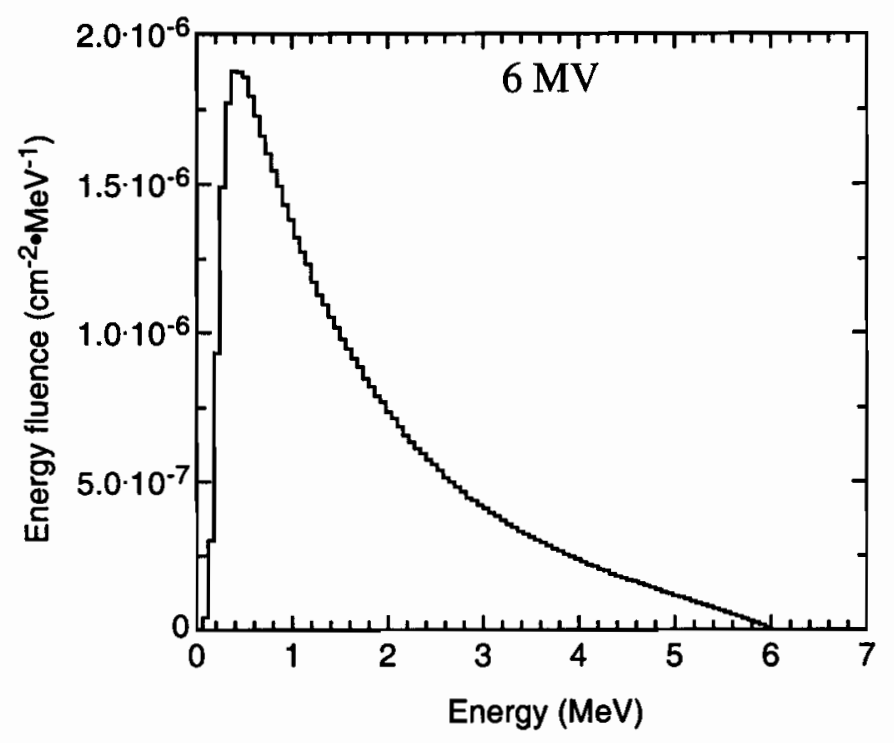

Figure 6-17. Photon spectrum (Clinac-2300 C/D) for a $6 \mathrm{MV}$ photon beam (SSD $=100 \mathrm{~cm}$, field size $=10 \times 10 \mathrm{~cm}^{2}$ ). The spectrum was calculated with Monte Carlo technique. 


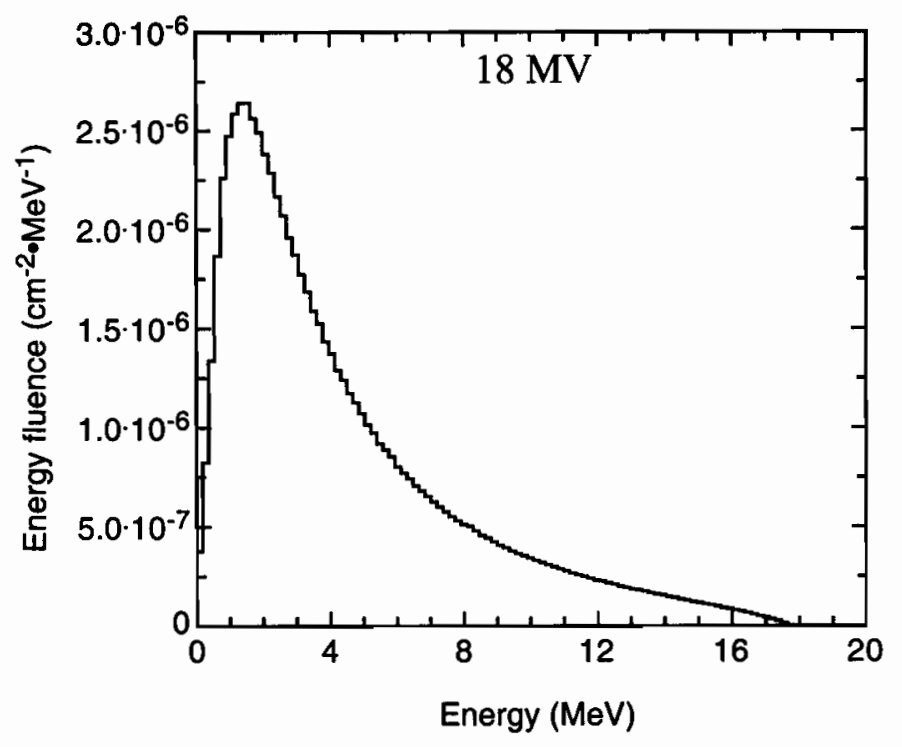

Figure 6-18. Photon spectrum (Clinac-2300 C/D) for a $18 \mathrm{MV}$ photon beam (SSD $=100 \mathrm{~cm}$, field size $=10 \times 10 \mathrm{~cm}^{2}$ ). The spectrum was calculated with Monte Carlo technique.

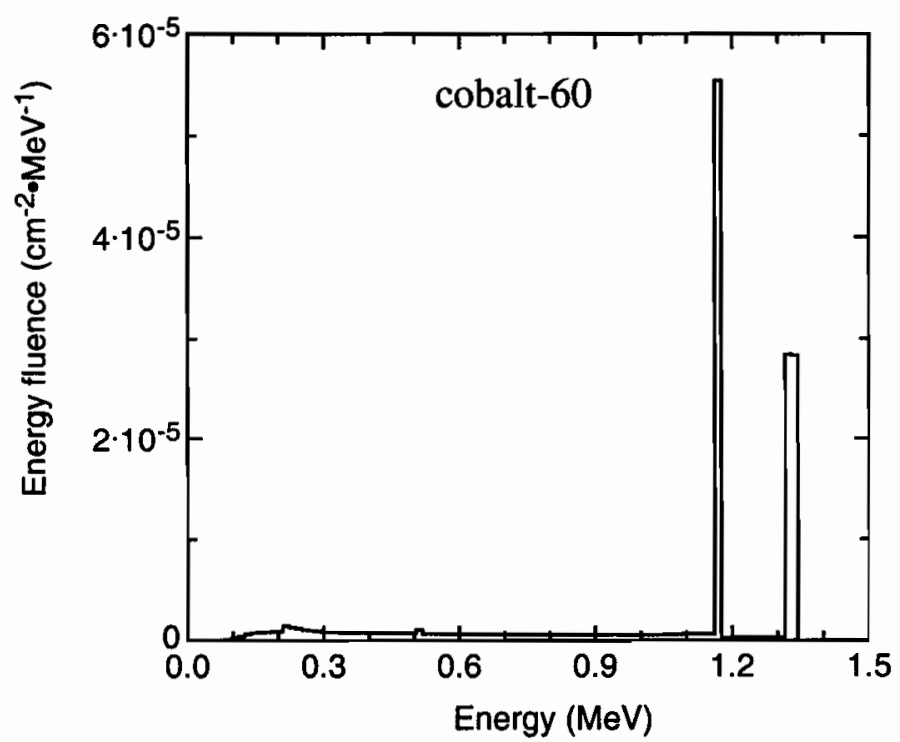

Figure 6-19. Photon spectrum (T-780) for a cobalt-60 photon beam (SSD $=80 \mathrm{~cm}$, field size $=10 \times 10 \mathrm{~cm}^{2}$ ). The spectrum was calculated with Monte Carlo technique. 


\section{IV.E. Mean restricted stopping power ratios}

SPRRZnrc/EGSnrc user code is used for calculating average restricted stopping power ratios. The configuration of interest, the PEEC sensitive volume, is an air cavity of size ranging from 2 to $3 \mathrm{~mm}$ in electrode separation. Therefore the threshold energy value should be between 9.1 to $11.3 \mathrm{keV}$, which represents the energy of secondary particles that would have just enough energy to cross a 2 and $3 \mathrm{~mm}$ cavity, respectively. The variation of mean restricted stopping power when varying the cut-off energy from 9 to $11 \mathrm{keV}$ is negligible and therefore we assumed a constant value of $10 \mathrm{keV}$ for the cut-off energy. PEGS4 cross section data with ICRU-37 density corrections ${ }^{31}$ was generated for Solid Water ${ }^{\mathrm{TM}}$, bone-equivalent material, and air with a cut-off of $10 \mathrm{keV}$. The cross section data was used along with the particular phase-space of the various beams as input to SPRRZnrc to calculate the mean restricted stopping power ratios as a function of depth for both material (SW and bone) to air.

\section{PEeC MONTE CARLo MOdel}

For performing Monte Carlo simulations within the PEEC geometry, a model of the chamber was produced using DOSRZnrc geometry and is illustrated in Fig. 6-20. Since the relevant measurements with PEEC are obtained along the central axis of the beam, and the PEEC lateral dimensions $\left(30 \times 30 \mathrm{~cm}^{2}\right)$ are larger than a typical calibration beam of $10 \times 10 \mathrm{~cm}^{2}$, it is a valid assumption to use a cylindrical model. Even though the PEEC has a square shape, no addition or lack of scatter is introduced by that assumption, since we are only concerned by calculations in regions along the central axis of the chamber or more specifically within the chamber air cavity. The specific materials for a given configuration of the chamber phantom and electrodes is taken into account as well as the specific electrode composition and thickness. The depth of measurement is adjusted so that the effective depth of measurement $d_{m}$ corresponds to the polarizing electrode surface, as shown in Fig. 6-20. The thickness of each electrode, $d_{c}$ and $d_{p}$, the thickness of the entrance window $d_{e}$, the size of the air cavity $d_{\text {air }}$, and the size of the piston add-on $t$ are all taken into con- 
sideration by the simulations. $r_{P E E C}$ is the size of the chamber and was assumed to be 15 $\mathrm{cm}$. $r_{\text {entry }}$ is the radius of the entrance window of the chamber, $r_{\text {elec }}$ is the radius of the polarizing electrode, $r_{\text {coll }}$ is the radius of the collecting electrode.

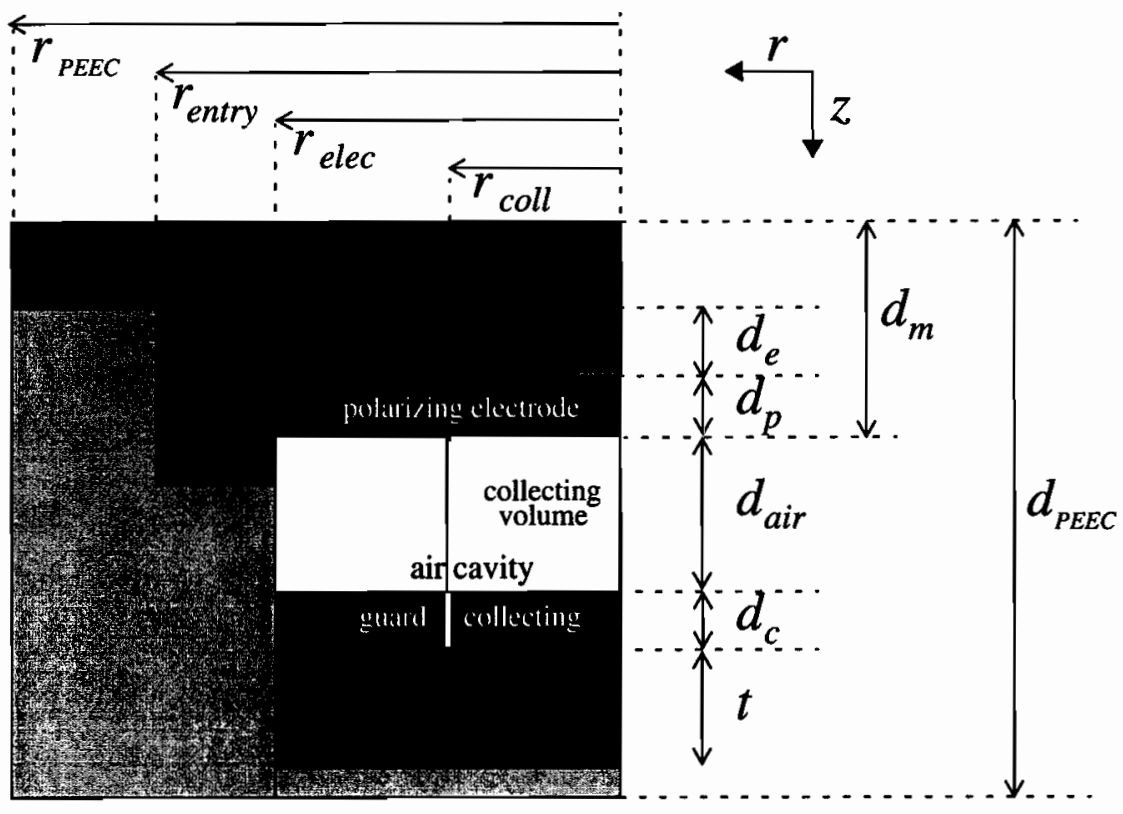

Figure 6-20. The simulated model of the PEEC in a right cylindrical geometry $(r, z)$ used for Monte Carlo with DOSRZnrc. The chamber is assumed to be cylindrical. The drawing is not to scale.

\section{Medical Physics Unit Monte Carlo computer CLUSTER}

Any quantity and its "type A" error calculated with BEAM/EGS4/EGSnrc Monte Carlo simulations is obtained through an average over a large number of particle histories. This principle enables parallel calculations, where a total number of histories to be simulated can be split into many smaller batches each of them having a different random number seed. Each batch can be run independently at a different time and on a different central processing unit (CPU). A cluster consisting of Pentium III duo-processor computers 
running Linux was assembled at the Medical Physics Unit. There are presently 10 machines (20 CPUs) in the cluster from which EGS4/EGSnrc simulations can be launched. A split number $n$ is given as a parameter of the simulation. The split number $n$ divides the total number of $N$ histories requested for the simulation and produces $n$ input files each of them having $N / n$ histories. These input files are used to start $n$ "jobs" distributed over the available CPUs of the cluster. Each input file uses different random seeds in order to produce different results for each batch, the random number generator providing a different random sequence for a different random seed. Jobs are distributed with the Network Queuing System (NQS) software ${ }^{40}$. At the completion of the jobs, the results of each batch are combined to obtain final results for $N$ histories and a reduction of computation time by a factor $\sim n$ is obtained.

Typical run time for a depth dose curve with relative uncertainties on the dose per incidence fluence less than $0.2 \%$, for a Solid Water ${ }^{\mathrm{TM}}$ or bone phantom with $2 \mathrm{~mm}$ voxels, is less than 2 hours using all 20 CPUs available. However when calculating dose to a small air voxel $(2 \mathrm{~mm})$ in geometries similar to Fig. 6-20, which include the contribution of the adjacent thin electrodes, the calculation time can extend up to 30 hours using all 20 CPUs. 


\section{References}

1 G. Comte de Buffon, "Essai d'arithmétique morale," in volume 4. Supplément à l'Histoire Naturelle (1777).

2 P. S. Laplace, "Theorie analytique des probabilités, Livre 2," in Oeuvres complètes de Laplace (L'académie des Sciences, Paris, 1886), Vol. 7, part 2, pp. 365-366.

3 Malvin H. Kalos and Paula A. Whitlock, Monte Carlo methods (J. Wiley \& Sons, New York, 1986).

4 Richard Rhodes, Dark sun: the making of the hydrogen bomb (Simon \& Schuster, New York, 1995).

5 Richard Rhodes, The making of the atomic bomb (Simon \& Schuster, New York, 1986).

6 S. M. Ulam and J. von Newmann, "On combination of stochastic and deterministic processes," Bull. Amer. Math. Soc. 53, 1120 (1947).

7 M. L. Goldberger, "The interaction of high energy neutrons and heavy nuclei," Phys. Rev. 74, 1269-1277 (1948).

8 R. R. Wilson, "Monte Carlo study of shower production," Phys. Rev. 86, 261-269 (1952).

9 J. C. Butcher and H. Messel, "Electron number distribution in electron-photon showers," Phys. Rev. 112, 2096-2106 (1958). 
10 A. A. Varfolomeev and I. A. Svetlolobov, "Monte Carlo calculations of electromagnetic cascades with account of the influence of the medium on bremsstrahlung," Soviet Physics JETP 36, 1263-1270 (1959).

11 G. Marsaglia and A. Zaman, “Toward a universal random number generator," Statistics and Probability letters 8, 35-39 (1990).

12 G. Marsaglia and A. Zaman, "A new class of random number generators," Annals of Applied Probability 1, 462-480 (1991).

13 M. Lüscher, “A portable high-quality random number generator for lattice field theory simulations," Computer Physics Communications 79, 100-110 (1994).

14 J. R. Ehrman, "The care and feeding of random numbers," SLAC VM Notebook, Module 19, SLAC Computing Services (1981).

15 D. E. Knuth, "Seminumerical algorithms," in The art of computer programming (Addison Wesley, Reading Mass., 1981), Vol. II.

16 D. E. Knuth, "Seminumerical algorithms," in The art of computer programming (Addison Wesley, Reading Mass., 1997), Vol. II.

17 F. James, "A review of pseudorandom number generators," CERN-Data Handling Division, Report DD/88/22 (1988).

18 F. James, "RANLUX: A FORTRAN implementation of the high-quality pseudo-random number generator of Lüsher," Computer Physics Communications 79, 111-114 (1994).

19 Theodore M. Jenkins, Walter R. Nelson, and Alessandro Rindi, Monte Carlo transport of electrons and photons (Plenum Press, New York, 1988). 
20 M. J. Berger and S. M. Seltzer, ETRAN, Monte Carlo code system for electron and photon transport through extended media, RISC computer code, package CCC-107 (Oak Ridge National Laboratory, Oak Ridge, TN, 1973).

21 J. A. Halblieb and T. A. Mehlhorn, "ITS: The Integrated TIGER Series of coupled electron/photon Monte Carlo transport codes," Sandia National Laboratory Report SAND 84-0573 (1984).

22 Judith F. Briesmeister, "MCNP - A General Monte Carlo N-Particle Transport Code," Report LA-13709-M, Los Alamos National Laboratory, Los Alamos (2000).

23 R. L. Ford and W. R. Nelson, "The EGS Code system - Version 3," Stanford Linear Accelerator Center Report SLAC-210 (Stanford Calif.) (1978).

24 W. R. Nelson, H. Hirayama, and D. W. O. Rogers, "The EGS4 Code system," Stanford Linear Accelerator Center Report SLAC-256 (Stanford Calif.) (1985).

25 D. W. Rogers, B. A. Faddegon, G. X. Ding et al., "BEAM: a Monte Carlo code to simulate radiotherapy treatment units," Med. Phys. 22 (5), 503-524. (1995).

D. W. O. Rogers, I. Kawrakow, J. P. Seuntjens et al., "NRC user codes for EGSnrc," Technical Report PIRS-702, National Research Concil of Canada, Ottawa, Canada (2000).

27 I. Kawrakow and D. W. O. Rogers, "The EGSnrc Code System: Monte Carlo simulation of electron and photon transport," Technical Report PIRS-701, National Research Concil of Canada, Ottawa, Canada (2000).

28 I. Kawrakow, "Accurate condensed history Monte Carlo simulation of electron transport. I. EGSnrc, the new EGS4 version,” Med. Phys. 27 (3), 485-498. (2000). 
29

I. Kawrakow, “Accurate condensed history Monte Carlo simulation of electron transport. II. Application to ion chamber response simulations," Med. Phys. 27 (3), 499-513. (2000).

30 M. J. Berger and S. M. Seltzer, "Stopping powers and ranges of electrons and positrons," Report NBSIR 82-2550-A, National Bureau of Standards, Washington D. C. (1983).

31 ICRU, "Stopping powers for electrons and positrons," ICRU Report 37, International Commission on Radiation Units and Measurements, Bethesda, Maryland (1984).

32 M. J. Berger, "Monte Carlo Calculation of the penetration and diffusion of fast charged particles," in Methods in Comput. Phys., edited by B. Alder, S. Fernbach and M. Rotenberg (Academic, New York, 1963), Vol. 1, pp. 135-215.

33 A. F. Bielajew, H. Hirayama, W. R. Nelson et al., "History, overview and recent improvements of EGS4," Technical Report PIRS-0436, National Research Concil of Canada, Ottawa, Canada (1994).

34 G. X. Ding, D. W. Rogers, and T. R. Mackie, "Calculation of stopping-power ratios using realistic clinical electron beams," Med. Phys. 22 (5), 489-501. (1995).

35 C. J. Karzmark, Craig S. Nunan, and Eiji Tanabe, Medical electron accelerators (McGraw-Hill Inc. Health Professions Division, New York, 1993).

36 E. B. Podgorsak, P. Metcalfe, and J. Van Dyk, "Medical Accelerators," in The modern technology of radiation oncology: a compendium for medical physicists and radiation oncologists, edited by J. Van Dyk (Medical Physics Pub., Madison, Wis., 1999), pp. $349-435$. 
37 G. X. Ding and D. W. Rogers, "Mean energy, energy-range relationships and depth-scaling factors for clinical electron beams," Med. Phys. 23 (3), 361-376. (1996).

38 D. W. O. Rogers, I. Kawrakow, and B. R. B. Walters, "Revised relationship between R50 and Eo for electron beams," proceedings of the $42^{\text {nd }}$ scientific meeting of the AAPM, Chicago, July 23-28, TU-E313-1, (2000).

39 M. Olivares, F. DeBlois, E. B. Podgorsak, and J. P. Seuntjens, "Electron Fluence Correction Factors for Various Materials in Clinical Electron Beams," Med. Phys. 28 (8), 1727-1734. (2001).

40 Stuart Herbert, Generic NQS v3.5 (http://www.gnqs.org/, Sheffield, UK, 1994-2000). 


\section{ChAPTER 7}

\section{Collection efficiency for ionization chambers in pulsed beams}

\section{INTRODUCTION}

Collection efficiency, an important parameter in absolute calibration of clinical megavoltage photon and electron beams with ionization chambers, is governed by ionic losses in the chamber sensitive volume through general recombination, initial recombination, and ionic diffusion against the electric field. The measurement of collection efficiency may be influenced adversely by charge multiplication and field emission in the chamber sensitive volume or by spurious signals produced by radiation in the chamber stem.

Procedures for determination of collection efficiency in ionization chambers have been studied for the past 100 years by numerous investigators. Boag ${ }^{1,2}$ is credited with the most notable contribution to the subject, both for continuous and pulsed radiation beams. His theoretical approach for air-filled ionization chambers exposed to continuous beams accounts for general recombination and ignores other possible charge-loss or charge-gain effects in the ionization chamber. The result for the near-saturation region is a linear relationship between $1 / I(V)$ vs. $1 / V^{2}$, with $I(V)$ the current measured in the chamber sensitive volume at a given chamber potential $V$. For pulsed radiation beams, on the other hand, Boag developed a model which accounts for general and initial recombination in the ionization chamber and results in a linear relationship between $1 / I(V)$ vs. $1 / V$. Extrapolation of the linear relationships to $1 / V^{2}=0$ and $1 / V=0$ yield the inverse of the chamber saturation current, $1 / I_{\text {sat }}$, for continuous and pulsed radiation beams, respectively. 
The assumption of a linear relationship of $1 / I(V)$ with $1 / V^{2}$ or $1 / V$ in the near-saturation region makes the determination of the saturation current $I_{\text {sat }}$ (and collection efficiency $f$ which is defined as $I(V) / I_{\text {sat }}$ ) simple in principle, since the linear relationship may be determined with only two measured points. This assumption forms the basis of the "two-voltage" techniques which are recommended by national and international radiation dosimetry protocols for determination of ionization chamber collection efficiencies in continuous as well as pulsed radiation beams.

Zankowski and Podgorsak ${ }^{3,4}$ have shown that for continuous radiation beams the linearity between $1 / I(V)$ and $1 / V^{2}$ actually breaks down in the extreme near-saturation region $(f>0.98)$ where chambers are normally operated when used in megavoltage machine output measurements. They observed the breakdown for various cylindrical and parallel plate ionization chambers and attributed the effect to a contribution of initial recombination and ionic diffusion, as well as to minute effects caused by the onset of charge multiplication in the chamber sensitive volume. Using an NE2571 chamber, they showed that this effect may cause an overestimate of the order of $0.4 \%$ in the determination of the chamber saturation current with the "two-voltage" technique.

Subsequently, Yang et al. ${ }^{5}$ have studied this effect on various commercial ionization chambers, also in continuous radiation beams, and observed a similar deviation from the linear relationship in the extreme near-saturation region. They speculated that at least a portion of the observed breakdown may be attributed to a chamber stem effect. Ideally, only the radiation interacting with the sensitive ionization chamber volume should contribute to the ionization reading. In practice, however, the radiation field will also irradiate the chamber stem and the adjoining cable, possibly increasing the measured signal because of radiation-induced conductivity or leakage occurring in the cable insulators inside the stem of the chamber. This spurious signal will likely be proportional to the polarizing voltage of the ionization chamber and will not only depend on the radiation field size but also on the beam geometry, chamber design, and radiation type.

In this chapter we show that, at high polarizing voltages, ionization chambers irradiated with pulsed radiation beams also show deviations from the assumed linear behavior, which for pulsed beams is $1 / Q$ vs. $1 / V$. This irregular behavior for chambers in pulsed radiation beams was first reported by Derikum and Roos ${ }^{6}$. Thus the standard 
"two-voltage" technique, just like in the case of continuous beams, may also produce an error in saturation currents and in absolute beam output calibrations. Similar to the case of continuous radiation beams, we attribute the breakdown in the $1 / I(V)$ vs. $1 / V$ linear relationship at least partially to the onset of charge multiplication in the ionization chamber sensitive volume at relatively high operating potentials applied to ionization chambers and partially to a chamber stem effect. Saturation currents and collection efficiencies for use with radiation dosimetry protocols can be determined from the measured data through incorporating into Boag's equation an exponential term which describes the charge multiplication and stem effect.

\section{MATERIAL AND METHODS}

\section{II.A. Ion collection efficiency for pulsed radiation beams}

Boag $^{7}$ has shown that the collection efficiency $f$ for ionization chambers in pulsed radiation beams may be expressed as:

$$
f=\frac{I(V)}{I_{\text {sat }}}=\frac{1}{u} \ln (1+u)
$$

where $u=\frac{\Lambda_{p}}{V}$, with $V$ the polarizing voltage and $\Lambda_{p}=\mu r d^{2}$. In the expression for $\Lambda_{p}$, $\mu$ is a constant equal to $3.02 \times 10^{10} \mathrm{VmC}^{-1}$ (ICRU 34) ${ }^{8} ; r$ the initial charge density per pulse; and $d$ the equivalent electrode spacing for the chamber, given by $(a-b)^{2} k_{c y l}^{2}$ for a cylindrical chamber where $k_{c y l}=\sqrt{\frac{(a+b) \ln (a / b)}{2(a-b)}}$ and $a$ and $b$ are the outer and inner electrode radius, respectively. Equation $(7-1)$ is valid when the radiation pulses are much shorter than the transit time of ions between the chamber electrodes so that there is no overlap of signal pulses. The transit time of ions is about $50 \mu \mathrm{s}$, while the linac pulses are on the order of a few microseconds.

For $f>0.9$ and for small charge densities $r$ the quantity $u$ becomes sufficiently small $(<0.1)$ to allow the following expansion of Eq. (7-1): 


$$
f^{-1}=\frac{u}{\ln (1+u)} \approx 1+\frac{u}{2}-\ldots
$$

or

$$
\frac{1}{I(V)} \approx \frac{1}{I_{s a t}}+\frac{\lambda_{p}}{V}
$$

with $\lambda_{p}=\frac{\Lambda_{p}}{2 I_{\text {sat }}}$. Thus, at a given polarizing voltage $V$ the inverse of the saturation current, $I_{\text {sat }}^{-1}$, and the collection efficiency $f$ can be determined by plotting the measured $1 / I(V)$ vs. $1 / V$ and extrapolating the data for $f>0.90$ to $1 / V=0$. A simplified version of this extrapolation is the so-called "two-voltage" technique 9,10 which is used to determine the collection efficiency and saturation current with ionization chamber currents measured in pulsed beams at only two polarizing voltage points in the near-saturation region. If $I_{H}$ and $I_{L}$ are the currents measured at a high polarizing voltage $V_{H}$ and a low polarizing voltage $V_{L}$, respectively, then $f\left(V_{H}\right)$, the collection efficiency at $V_{H}$, is given by:

$$
f\left(V_{H}\right)=\frac{I_{H}}{I_{s a t}} \approx \frac{I_{H} / I_{L}-V_{H} / V_{L}}{1-V_{H} / V_{L}}
$$

The AAPM TG-21 protocol $^{9}$ which deals with the calibration of photon and electron beams presents a further simplification of Eq. (7-4) using $V_{H}=2 V_{L}$ (normally $V_{L}$ and $V_{H}$ of 150 and $300 \mathrm{~V}$, respectively, are used for clinical beam calibration), so that Eq. (7-4) for pulsed radiation beams reduces to:

$$
f\left(V_{H}\right)=2-\frac{I_{H}}{I_{L}}
$$

or

$$
I_{s a t}=\frac{I_{H} I_{L}}{2 I_{L}-I_{H}}
$$

A subsequent clarification ${ }^{10}$ of the AAPM TG-21 protocol recommended the use of a polarizing voltage ratio $V_{H} / V_{L}$ greater than 2 for the determination of $f$ and $I_{s a t}$, mainly 
because in many commercially available instruments it is not possible to halve the chamber bias potential. For $\left(V_{H} / V_{L}\right)>2$ the clarification ${ }^{10}$ recommends the use of data and methods that were provided by Weinhous and Meli ${ }^{11}$.

\section{II.B. Equipment and techniques}

Saturation curves were measured for two pulsed photon radiation beams ( 6 and $18 \mathrm{MV}$ ) and two pulsed electron beams ( 9 and $18 \mathrm{MeV}$ ). All photon and electron pulsed beams were generated by a clinical linear accelerator (Clinac $2300 \mathrm{C} / \mathrm{D}$; Varian, Palo Alto, CA). Three commercial Farmer-type, $0.6 \mathrm{~cm}^{3}$ ionization chambers (one model PR0-6C; Capintec, Ramsey, NJ and two model NE2571; Nuclear Enterprises, Beenham, Reading, England) were used in our experiments; the first as the measurement chamber and the second two as both a reference chamber and a measurement chamber.

The measurement chambers (PR0-6C and NE2571) were operated in the standard configuration; the collecting (inner) electrode was grounded through an electrometer (model 35617; Keithley, Cleveland, $\mathrm{OH}$ ) and the polarizing (outer) electrode was biased with a variable, computer-controlled high voltage power supply (model 648; Keithley, Cleveland, $\mathrm{OH}$ ). The reference chamber (NE2571) was also operated in the standard configuration, and was grounded and biased by another electrometer (model 35040; Keithley, Cleveland, $\mathrm{OH})$. The measurement chamber polarizing potential was varied from 5 to 1050 $\mathrm{V}$ (for the PR0-6C chamber) and up to $700 \mathrm{~V}$ (for the NE2571 chamber), the reference chamber potential was kept constant at $-300 \mathrm{~V}$.

The two electrometers and the variable power supply were interfaced with a personal computer (IBM-PC compatible 486) with an IEEE-488 interface. An in-house developed computer program controlled the acquisition of saturation curve data, allowing a real time display and statistical treatment of the current or charge, measured with the measurement chamber at a given chamber polarizing voltage as a function of time.

In the photon beam mode the beam output of the linac is stabilized with the so-called "pulse width method" through varying individual pulse widths. This makes the linac output stable throughout the delivery of a given dose and allows the measurement of 
ionization chamber signals in the differential (current) mode. The chamber current readings (on the order of $1 \mathrm{nA}$ ) were measured every one second and averaged over at least 100 readings. We repeated the procedure when the standard deviation on the readings yielded a relative error greater than $0.1 \%$ of the average value. The readings of the measurement chamber were normalized to readings of the reference chamber to insure that minute variations in the linac output did not affect the measured signal.

In the electron beam mode, the output of the linac is stabilized with the so-called "pulse drop method" through dropping, as required, individual pulses in the pulse sequence. Therefore, the linac output and with it the current measured in the ionization chamber can vary quite significantly with time during a given irradiation. We therefore measured the collection efficiency in electron beams in the integral (charge) mode. The mean of at least 10 charge measurements was taken and the relative error on the average of the measurement was kept below $0.3 \%$.

The variations in the linac output were minimal during the time of measurement of a full saturation curve, with typical variations on the average measured current of less than $0.1 \%$ for photon beams and typical variations on the average measured charge of less than $0.3 \%$ for electron beams. The variations were accounted for through the use of the reference chamber.

Following the chamber polarizing voltage switching, a relatively long approach to a new stabilization current or charge value was observed, most likely because of polarization effects in the chamber thimble material. The time to attain a new current or charge level was inversely proportional to the dose rate and proportional to the polarizing voltage difference between the previous and new point of measurement as well as to the time of irradiation at the previous polarizing voltage level. Approach times for stabilization of up to 15 minutes at $400 \mathrm{cGy} / \mathrm{min}$ were observed. During a saturation curve measurement, we periodically re-stabilized the chamber to certain polarizing voltage values $(50,150,300 \mathrm{~V})$ to verify the reproducibility which was measured to be $0.1 \%$ and $0.3 \%$ for photon and electron beam measurements, respectively.

Saturation curves were obtained for both polarities of the polarizing voltage, and the polarity effect was negligible for polarizing voltages above $20 \mathrm{~V}$. Leakage current in the absence of radiation was linear with polarizing voltage and amounted to less than $0.1 \%$ 
of the measured current at the chamber potential of $1050 \mathrm{~V}$ for the lowest dose rate used (40 cGy/min).

Photon beam measurements with the PR0-6C chamber were carried out in a polystyrene phantom at a depth between 3.5 and $5 \mathrm{~cm}$. The source-chamber distance (SCD) and the linac dose rate setting were adjusted to obtain various dose rates ranging from 40 $\mathrm{cGy} / \mathrm{min}$ (long SCD, phantom on the treatment room floor, linac output set at $100 \mathrm{MU} / \mathrm{min}$ ) to $\sim 600 \mathrm{cGy} / \mathrm{min}$. Most photon beam measurements were carried out with the field size fixed at $10 \times 10 \mathrm{~cm}^{2}$ at $\mathrm{SCD}$; however, to evaluate the influence of field size on the saturation curve, we also measured saturation curves for $18 \mathrm{MV}$ pulsed photon beams with a fixed SCD of $100 \mathrm{~cm}$ and a dose rate of $400 \mathrm{cGy} / \mathrm{min}$ at two different field sizes: $10 \times 10 \mathrm{~cm}^{2}$ and $30 \times 30 \mathrm{~cm}^{2}$.

Electron beam measurements with the PR0-6C chamber were carried out with a $7 \mathrm{~mm}$ aluminum build-up cap in air. The use of a metallic build-up cap simplified the stabilization of the measured signal, since charge buildup in the standard plastic cap material caused by the electron beam was thereby avoided ${ }^{12}$. The field size was fixed to $10 \times 10 \mathrm{~cm}^{2}$ at a fixed SCD of $100 \mathrm{~cm}$.

Saturation curves were also measured in an $18 \mathrm{MV}$ pulsed photon beam with a Holt parallel plate chamber (Memorial Hospital, New York 10021) ${ }^{13}$ and an NE2571 ionization chamber. The Holt chamber was embedded in a polystyrene phantom with the polarizing electrode at a depth of $3 \mathrm{~cm}$. A source-surface distance of $100 \mathrm{~cm}$, dose rate of $400 \mathrm{cGy} / \mathrm{min}$, and two field sizes $\left(3 \times 3 \mathrm{~cm}^{2}\right.$ and $\left.15 \times 15 \mathrm{~cm}^{2}\right)$ were used. Saturation curves with the NE2571 chamber were measured at two different field sizes $\left(10 \times 10 \mathrm{~cm}^{2}\right.$ and $30 \times 30 \mathrm{~cm}^{2}$ ) at a dose rate of $\sim 400 \mathrm{cGy} / \mathrm{min}$. The chamber was placed in a SolidWater ${ }^{\mathrm{TM}}$ phantom at a depth of $5 \mathrm{~cm}$ and an SSD of $100 \mathrm{~cm}$ was used.

Finally, saturation curves were also measured in an $18 \mathrm{MV}$ pulsed photon beam with the phantom-embedded extrapolation chamber (PEEC) with a NE2571 ionization chamber as a reference chamber. The PEEC chamber was operated in Solid Water ${ }^{\mathrm{TM}}$ mode with the graphite electrodes with a gap of $2.5 \mathrm{~mm}$ and was positioned such the depth of measurement was $10 \mathrm{~cm}$. A source-surface distance of $100 \mathrm{~cm}$, dose rate of $400 \mathrm{cGy} / \mathrm{min}$, and two field sizes $\left(5 \times 5 \mathrm{~cm}^{2}\right.$ and $\left.10 \times 10 \mathrm{~cm}^{2}\right)$ were used. 


\section{RESULTS AND DISCUSSION}

In Fig. 7-1(a) we show an example of a saturation curve for an $18 \mathrm{MV}$ pulsed beam measured at a dose rate of $\sim 400 \mathrm{cGy} / \mathrm{min}$ with the cylindrical PR0-6C ionization chamber. The saturation curve data are plotted in the form $1 / I$ vs. $1 / V$ in the collection efficiency range $f>0.85$, corresponding to an applied polarizing voltage range from 10 to $1050 \mathrm{~V}$. The linearity of data points seems excellent, confirming the validity of Boag's equation [Eq. (7-3)]; however, at very high polarizing voltages, there is a deviation of data points from the linearity predicted by Eq. (7-3) similar to the previously found behavior in continuous radiation beams ${ }^{3}$. The solid line in Fig. 7-1(a) encompasses the data points which are in the polarizing voltage region between 20 and $100 \mathrm{~V}$, suitable for use in the linear fitting and subsequent linear extrapolation to $1 / V=0$.

A closer look at the high polarizing voltage region of Fig. 7-1(a) is shown in Fig. 7-1(b), with data from the shaded region of Fig. 7-1(a) covering $f>0.98$, plotted on an expanded scale. The extrapolation of the linear fit of Fig. 7-1(a) to $1 / V=0$ is shown with the solid line resulting in an $I_{s a t}$ of $1.500 \mathrm{nA}$. The dashed line, which represents the "two-voltage" technique using the standard 150 and $300 \mathrm{~V}$ as the two polarizing voltage points in Eq. (7-6), results in $I_{\text {sat }}=1.508 \mathrm{nA}$, exceeding the linearly extrapolated value of $1.500 \mathrm{nA}$ by about $0.5 \%$. The deviation of data points from linearity increases exponentially with an increasing polarizing voltage, i.e., with a decreasing $1 / V$, and the data points at polarizing voltages above $300 \mathrm{~V}$ actually exceed the saturation current obtained through the extrapolation of the linear fit of Fig. 7-1(a) toward $1 / V=0$.

Boag et al. ${ }^{14}$ have recently discussed the effects of free-electron collection on recombination correction to ionization measurements of pulsed radiation. Free electrons, by virtue of their higher mobility than that of ions, will affect the $1 / I$ vs. $1 / V$ linearity; however, they cannot produce currents which exceed the saturation current $I_{\text {sat }}$. 

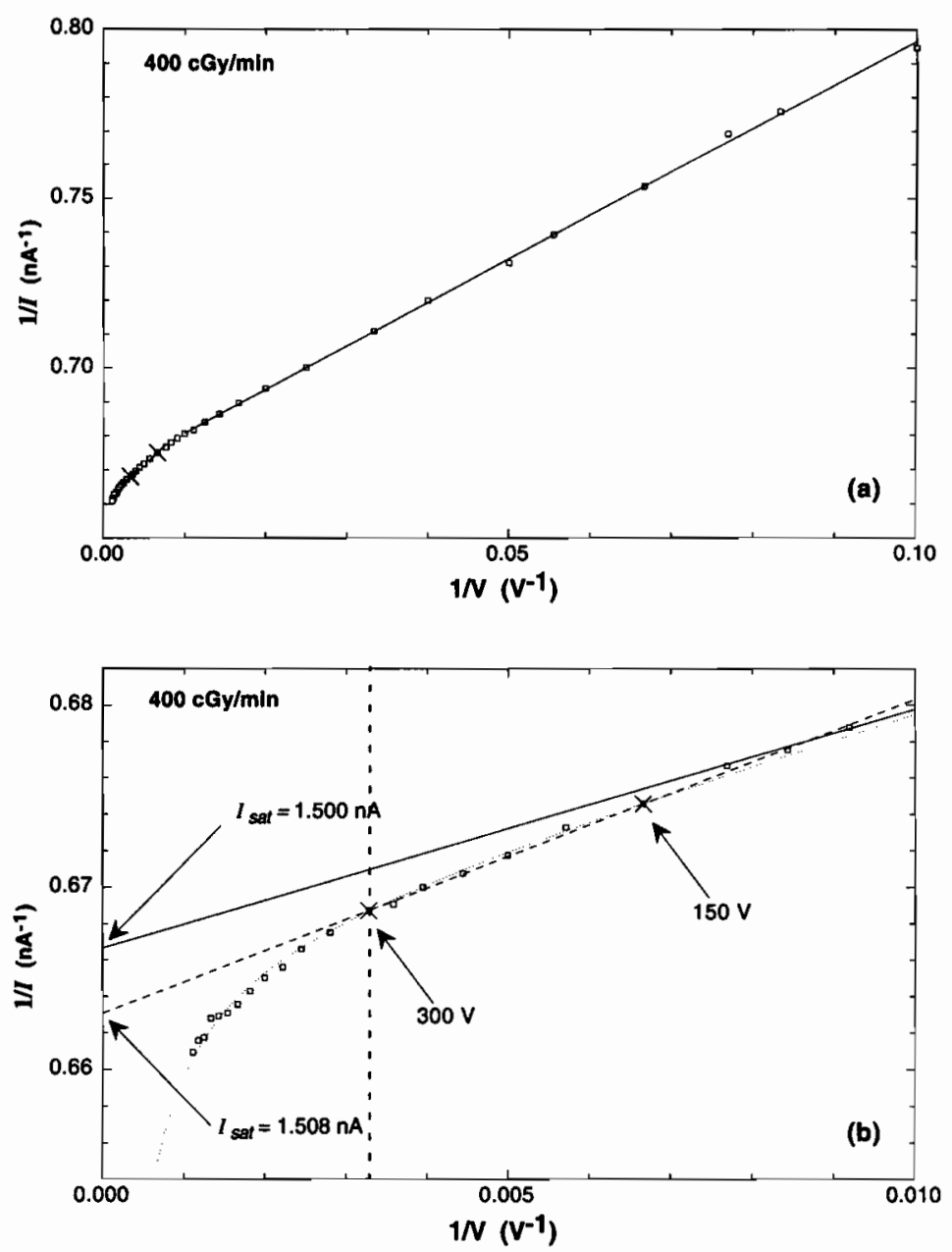

Figure 7-1. Saturation data measured with an ionization chamber (PR0-6C) in an $18 \mathrm{MV}$ pulsed photon beam at $\sim 400 \mathrm{cGy} / \mathrm{min}$. Part (a) shows data in the polarizing voltage region from $\sim 10$ to $\sim 1050 \mathrm{~V}$, with the solid line encompassing points used in linear extrapolation to $1 / V=0$. Part (b) shows the shaded region of part (a) on an expanded scale, with the solid line representing the linear extrapolation of data shown in part (a), the dashed line representing the "two-voltage" technique through 150 and $300 \mathrm{~V}$ points, represented by the $x$ points, and the dotted curve representing Eq. (7-7) with $\lambda_{p}=1.31 \mathrm{~V} / \mathrm{nA}$ and $\gamma=1.37 \times 10^{-5} \mathrm{~V}^{-1}$.

We now postulate that the deviation of data points from linearity arises from the onset of charge multiplication in the chamber sensitive volume or from some other polarizing voltage-dependent effect. Similarly to the situation with the continuous radiation 
beams ${ }^{3}$, we account for this effect through an exponential term $\mathrm{e}^{\gamma V}$, where $\gamma$ is a parameter. Equation (7-3) can then be written as follows:

$$
\frac{1}{I} \approx\left[\frac{1}{I_{\text {sat }}}+\frac{\lambda_{p}}{V}\right] e^{-\gamma V}
$$

The dotted curve in Fig. 7-1(b) represents a least square fit of measured data to Eq. (7-7) with $\gamma=1.48 \times 10^{-5} \mathrm{~V}^{-1}$ and $\lambda_{p}=1.29 \mathrm{~V} / \mathrm{nA}$. The curve agrees with measured data very well in the whole range of polarizing voltages used in the experiment (10 to $1050 \mathrm{~V}$ ), supporting the validity of combining the charge-loss effects of general recombination with the charge-gain effects modelled with the exponential term $e^{\gamma V}$. The $I_{\text {sat }}$ determined from Eq. (7-7) is equal to $1.500 \mathrm{nA}$ which is identical to the value determined through a linear fit to measured data using measured points at relatively low polarizing voltages (10 to $100 \mathrm{~V}$ ), and extrapolating to $V \rightarrow \infty$, i.e., $1 / V=0$.

The data of Fig. 7-1 were measured with an $18 \mathrm{MV}$ pulsed radiation beam at $\sim 400 \mathrm{cGy} / \mathrm{min}$ with a field size of $10 \times 10 \mathrm{~cm}^{2}$. Measurements of saturation curves for pulsed photon beams at other dose rates and other energies, as well as for electron beams of various dose rates and energies have all revealed behavior similar to that shown in Fig. 7-1; a breakdown of $1 / I$ vs. $1 / V$ linearity at large polarizing voltages, that is, in the polarizing voltage region where ionization chambers are normally operated in radiation dosimetry. Examples of saturation curves for various photon and electron beams (field size: 10x10 $\mathrm{cm}^{2}$ ) are shown in Fig. 7-2 and Fig. 7-3.

Figure 7-2 shows saturation curves for an $18 \mathrm{MV}$ beam for various dose rates in the range from 40 to $600 \mathrm{cGy} / \mathrm{min}$ and Figure 7-3 shows data for 6 and $18 \mathrm{MV}$ photon beams and 9 and $18 \mathrm{MeV}$ electron beams at a dose rate of $\sim 400 \mathrm{cGy} / \mathrm{min}$. The electron data, although measured in the charge mode, was converted to current for the analysis. The right-hand columns of Figures 7-2 and 7-3 show on an expanded scale the data of the left hand column, identified with the shaded area. As in Fig. 7-1, the solid lines in Figures 7-2 and 7-3 represent the linear fit of Eq. (7-3) to data measured at relatively low polarizing voltages; the dashed lines represent the "two-voltage" technique through the standard 150 and $300 \mathrm{~V}$ points [Eq. (7-6)]; and the dotted curves represent the fitting of Eq. (7-7) to mea- 
sured data. It is evident that the semi-empirical model, represented by Eq. (7-7), describes the measured data well not only in the extreme near-saturation region but generally in the whole collection efficiency range above 0.90 (polarizing voltage range from 20 to $1050 \mathrm{~V}$ ) for a large variety of beam energies and dose rates of pulsed photon and electron beams.
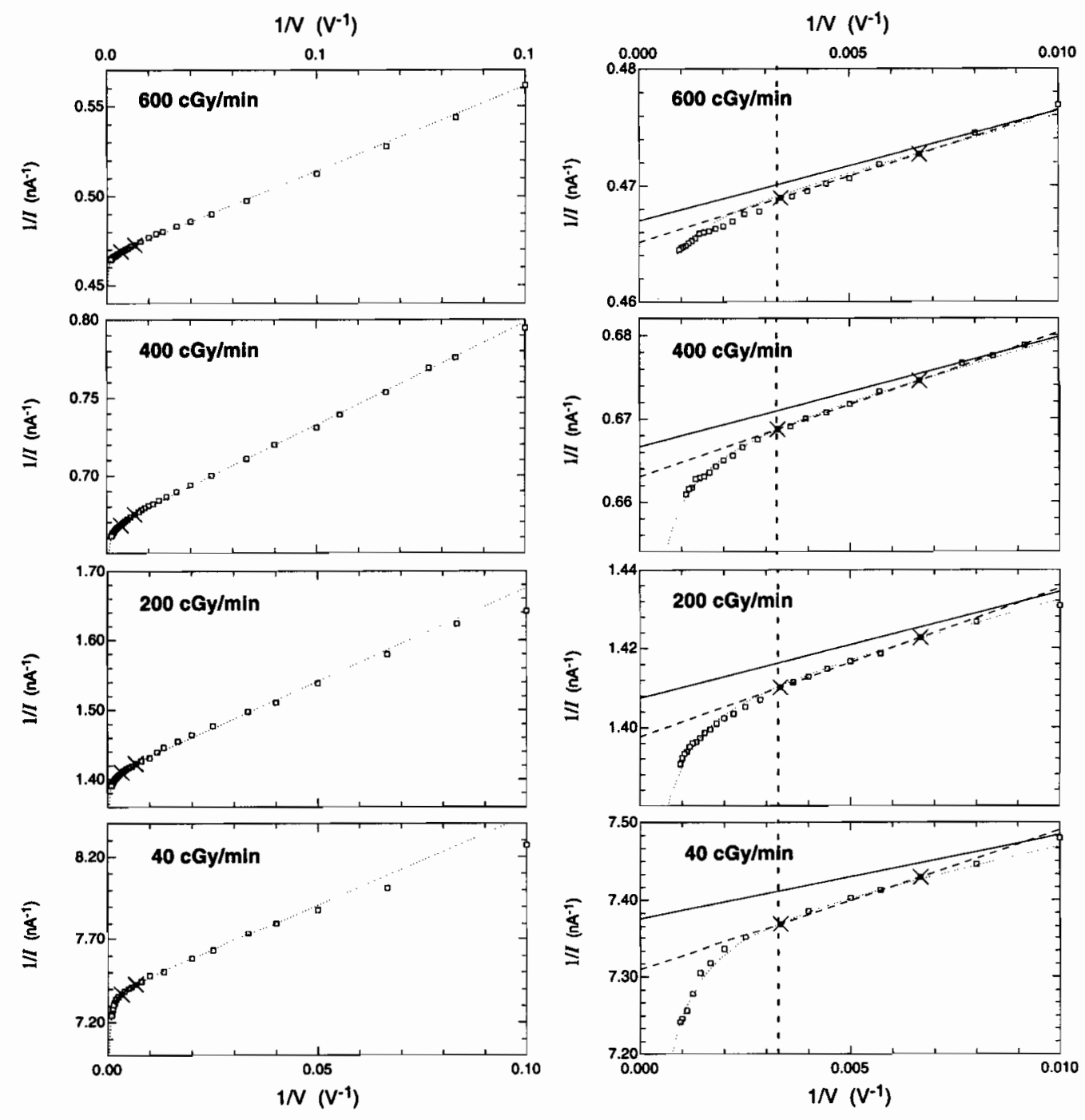

Figure 7-2. Saturation data measured with an ionization chamber (PR0-6C) in an $18 \mathrm{MV}$ pulsed photon beam at various dose rates in the polarizing voltage region from $\sim 10$ to $\sim 1050 \mathrm{~V}$. The graphs on the right represent data from the shaded region on the left. The dotted curves represent Eq. (7-7) with appropriate parameters $\lambda_{p}$ and $\gamma$ given in Table 7-1; the dashed lines represent the "two-voltage" technique; and the solid lines represent linear extrapolation of data in the region $0.90<f<0.98$ to $1 / V=0$. The symbols $\mathrm{X}$ represent the 150 and $300 \mathrm{~V}$ points on the graphs. 

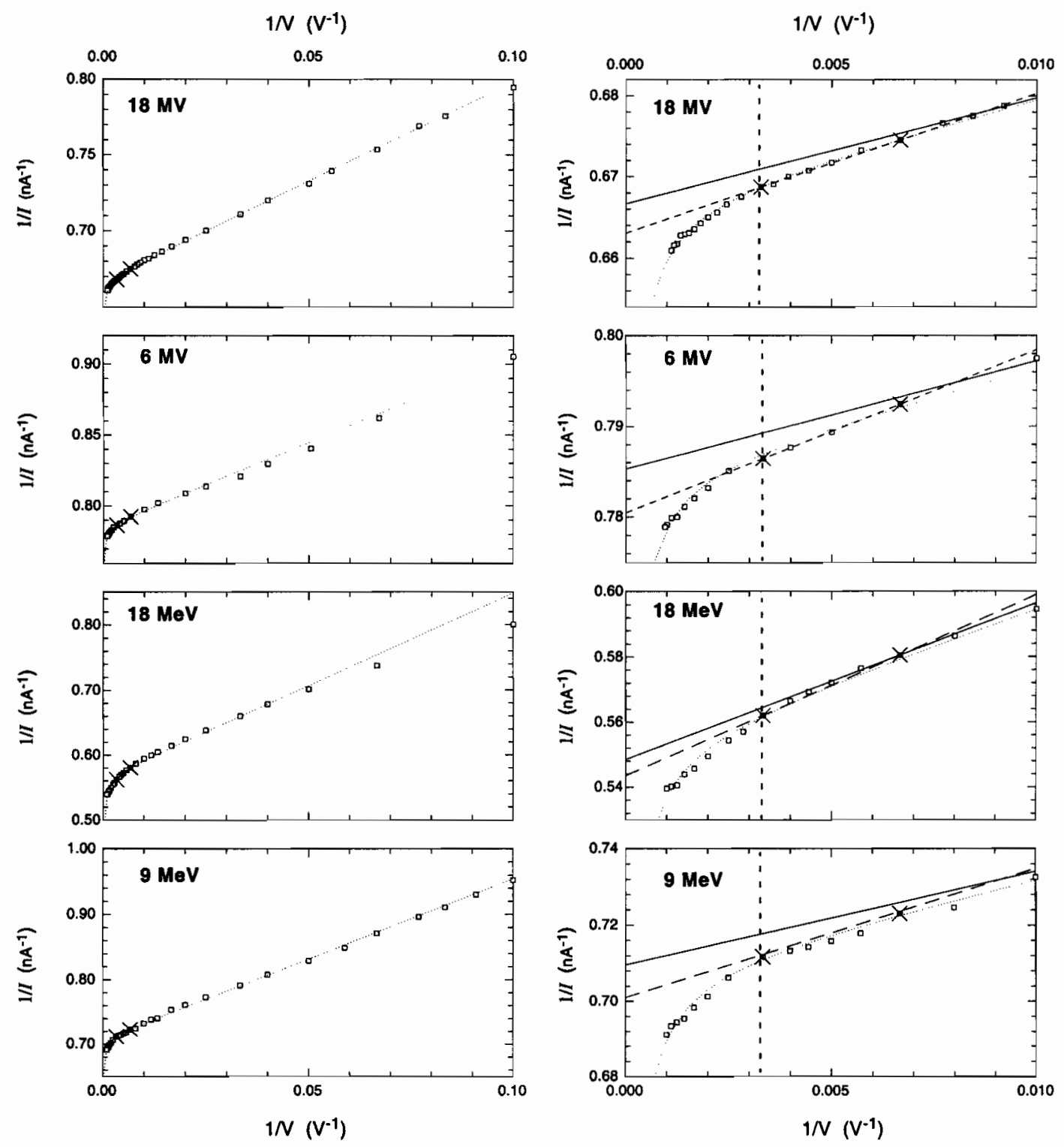

Figure 7-3. Saturation data measured with an ionization chamber (PR0-6C) in pulsed 6 and 18 MV photon beams and 9 and $18 \mathrm{MeV}$ electron beams at $\sim 400 \mathrm{cGy} / \mathrm{min}$ in the polarizing voltage region from $\sim 10$ to $\sim 1050 \mathrm{~V}$. The graphs on the right represent data from the shaded region on the left. The dotted curves represent Eq. (7-7) with parameters $\lambda_{p}$ and $\gamma$ given in Table 7-1; the dashed lines represent the "two-voltage" technique; and the solid lines represent linear extrapolation of data in the region $0.90<f<0.98$ to $1 / V=0$. The symbols $\mathrm{x}$ represent the 150 and $300 \mathrm{~V}$ points on the graphs. 
In Table 7-1 we give a summary of saturation current data for 6 and $18 \mathrm{MV}$ pulsed photon beams as well as for 9 and $18 \mathrm{MeV}$ pulsed electron beams at various dose rates. Saturation currents were determined with three methods: (a) extrapolation of linear low polarizing voltage $1 / I$ vs. $1 / V$ data to $1 / V=0$, (column 3 ); (b) standard "two-voltage" technique with $V_{L}=150 \mathrm{~V}$ and $V_{H}=300 \mathrm{~V}$, (column 4); and (c) fitting of measured data to Eq. (7-7), (column 5), using appropriate values for $\lambda_{p}$ and $\gamma$ listed in columns 6 and 8, respectively. Similarly to findings in Fig. 7-1, methods (a) and (c) give essentially the same results for $I_{\text {sat }}$, as shown in column 9 of Table 7-1 by the very small percent difference between saturation currents determined with the two methods. On the other hand, as shown in column 10 of Table 7-1, saturation currents determined with the "two-voltage" technique consistently exceed by $\sim 0.5 \%$ or more the saturation currents determined with Eq. (7-7), suggesting that the use of the "two-voltage" technique may introduce errors of the order of $0.7 \%$ in absolute dosimetry of pulsed photon and electron beams.

According to Eq. (7-3), the product $\lambda_{p} I_{\text {sat }}$ as well as the parameter $\gamma$ should be constant and independent of the dose rate for a given chamber and a given radiation beam. Columns (7) and (8) of Table 1 show that this may hold within the constraints of the experimental setup and measurement uncertainties. Column (8) actually indicates substantial variations in $\gamma$ with dose rate; however, the uncertainties on $\gamma$ are quite large, because it is determined from only a small number of measurements points at high polarizing voltages. The risk of chamber insulation or air breakdown obviously limits the polarizing voltage range for $\gamma$ measurement to relatively low values at which the breakdown in $1 / I$ vs. $1 / V$ is still not very pronounced.

Recently, Yang et al. ${ }^{5}$ speculated that the non-linearity in the $1 / I$ vs. $1 / V^{2}$ saturation curve data in continuous beams results not only from the onset of charge multiplication in the chamber sensitive volume but also from a chamber stem effect. To evaluate the possibility of the non-linearity in pulsed beams resulting from spurious signals originating in the chamber stem rather than from the chamber sensitive volume, we measured saturation curves with the PR0-6C chamber in an $18 \mathrm{MV}$ photon beam using three different field sizes: (1) an open $10 \times 10 \mathrm{~cm}^{2}$ field; (2) an open $30 \times 30 \mathrm{~cm}^{2}$ field; and (3) a $10 \times 10 \mathrm{~cm}^{2}$ field with the chamber stem and connector shielded with $20 \mathrm{~cm}$ thick lead blocks. The field arrangements are shown schematically in Fig. 7-4 for the two open fields in part (a) and for 
the shielded stem in part (b). The chamber was at a depth of $3.5 \mathrm{~cm}$ in a polystyrene phantom and the SCD was $100 \mathrm{~cm}$.

Table 7-1. Parameters of saturation curves measured with a cylindrical Farmer-type ionization chamber (PR-06) in 6 and $18 \mathrm{MV}$ pulsed photon beams and in $9 \mathrm{MeV}$ and $18 \mathrm{MeV}$ pulsed electron beams (all field sizes are $10 \times 10 \mathrm{~cm} 2$ ). Column 3 is obtained by a linear fit to the data, Column 4 is obtained by Eq. (7-6) and Column 5 is obtained by Eq. (7-7). Column 9 is found by $100 \frac{(\operatorname{col}(3)-\operatorname{col}(5))}{\operatorname{col}(5)}$ and Column 10 is found by $100 \frac{(\operatorname{col}(4)-\operatorname{col}(5))}{\operatorname{col}(5)}$.

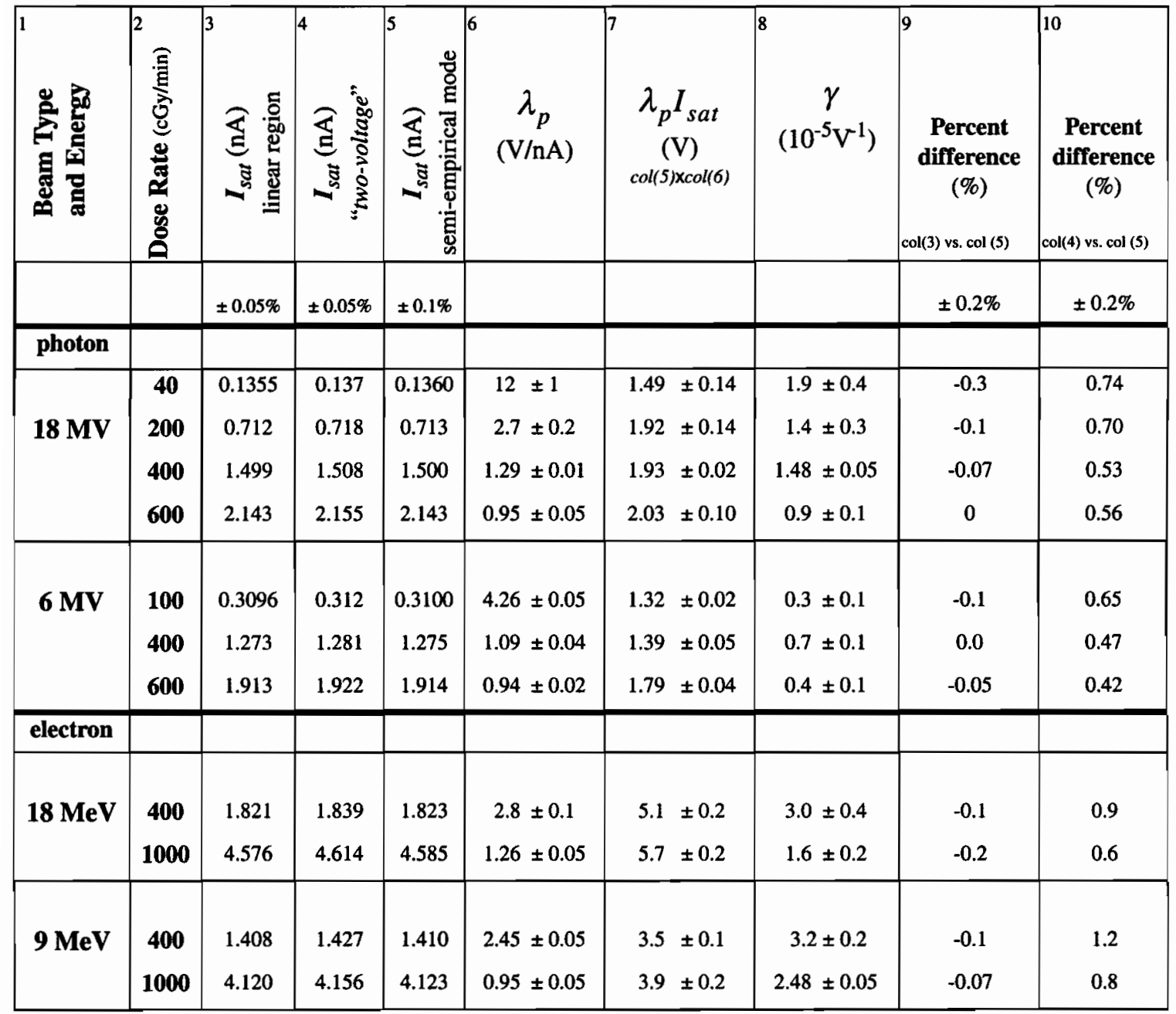




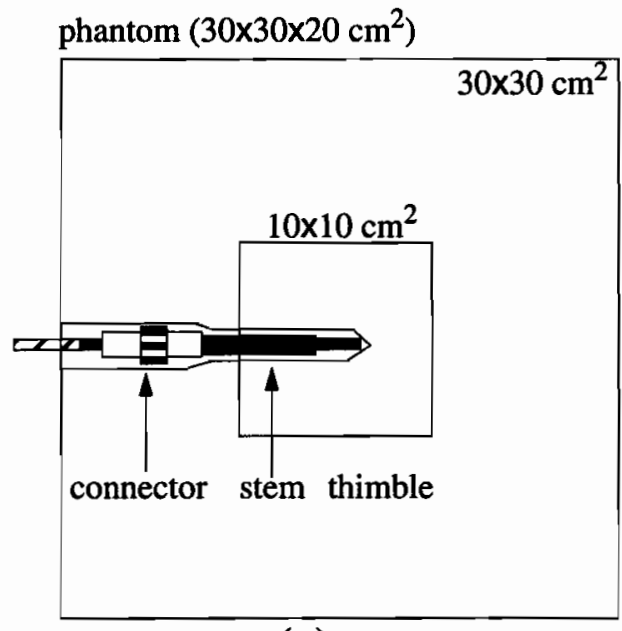

(a)

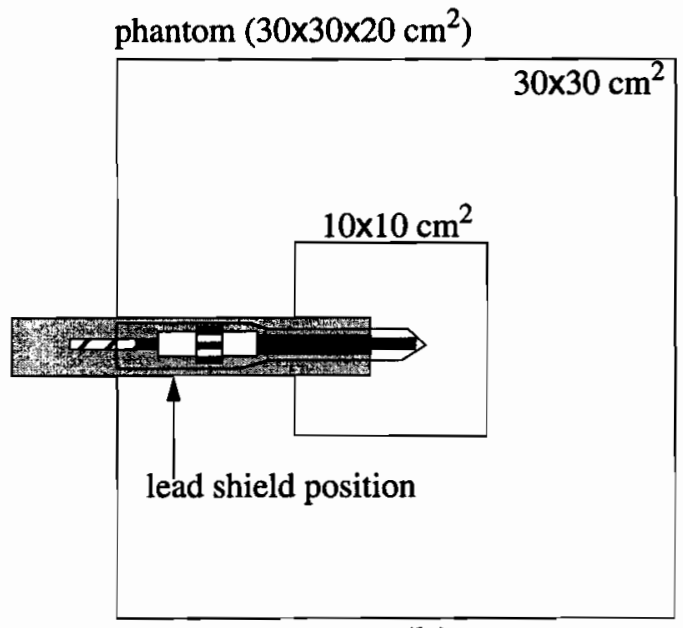

(b)

Figure 7-4. Schematic view of the three irradiation fields showing the position of the chamber, the field, and the shielding for measurements with the PR0-6C ionization chamber. Part (a) is for the $10 \times 10$ and $30 \times 30$ $\mathrm{cm}^{2}$ open fields, part (b) for the $10 \times 10 \mathrm{~cm}^{2}$ field with a shielded stem, connector and cable of the ionization chamber.

Figure 7-5(a) shows results of this experiment with saturation curves in the form $1 / I$ vs. $1 / V$ for the three fields of Fig. 7-4, and Table 7-2 lists the parameters associated with the three curves. The solid lines of Fig. 7-5(a) represent a fit to the linear portion of the saturation data; the dotted lines the "two-voltage" technique through the 150 and $300 \mathrm{~V}$ points; and the dashed curves the fit of measured data to Eq. (7-7) with parameters $\lambda_{p}$ and $\gamma$ given in Table 7-2. 


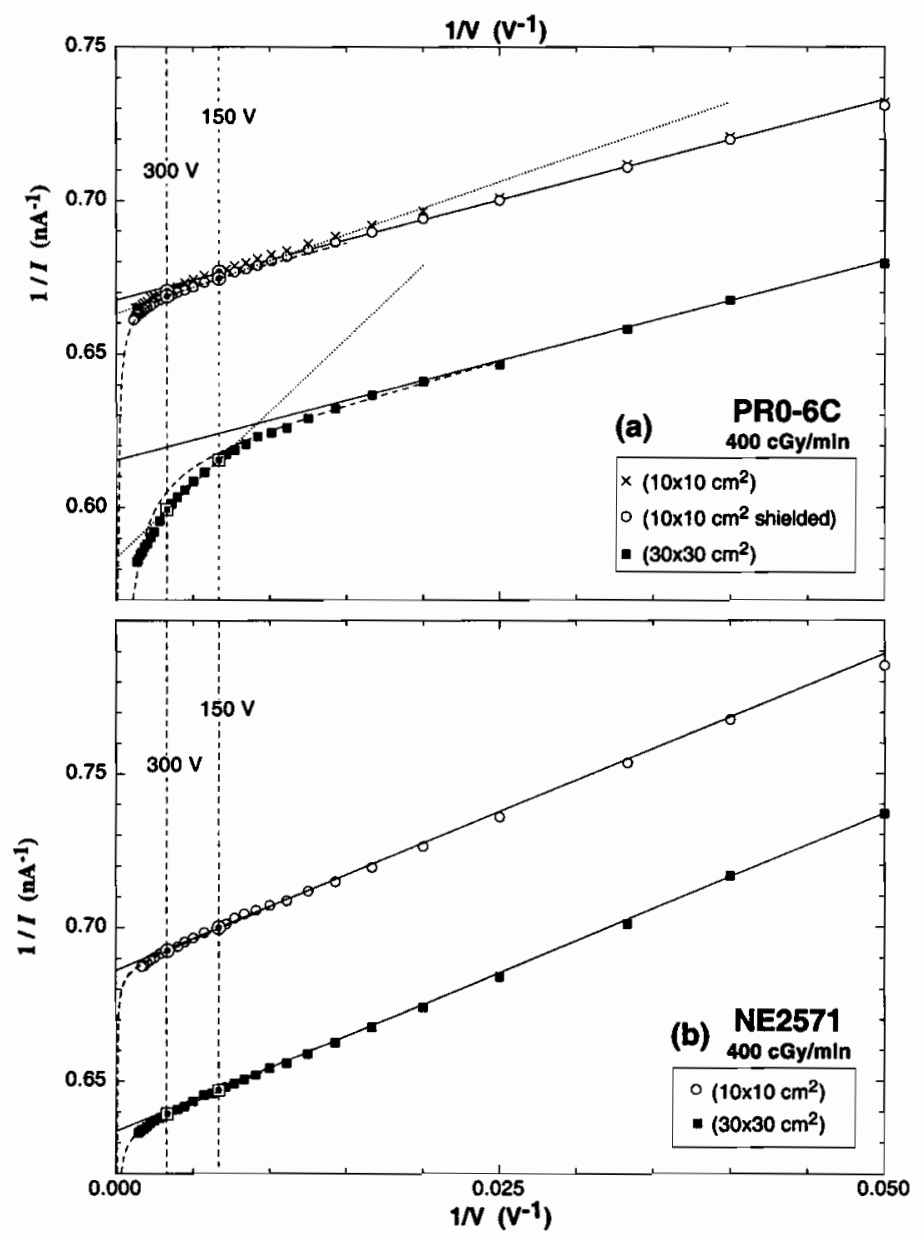

Figure 7-5. Saturation data measured with an ionization chamber (PR0-6C) in an $18 \mathrm{MV}$ pulsed photon beam at $\sim 400 \mathrm{cGy} / \mathrm{min}$ in the polarizing voltage range from $\sim 20$ to $\sim 1050 \mathrm{~V}$ with the three field arrangements shown in Fig. 7-4. The solid lines represent linear fits to the linear portion of the data; the dashed lines the fit of the data to Eq. (7-7); and the dotted lines the "two-voltage" technique. b) Saturation data measured with an ionization chamber (NE2571) in an $18 \mathrm{MV}$ pulsed photon beam at $400 \mathrm{MU} / \mathrm{min}$ in the polarizing voltage range from $\sim 20$ to $\sim 700 \mathrm{~V}$ for the two field sizes: $10 \times 10 \mathrm{~cm}^{2}$ and $30 \times 30 \mathrm{~cm}^{2}$. The solid lines are the linear fits to the linear portion of the data, the dashed lines are the fit of the data to Eq. (7-7). The position of the 150 and $300 \mathrm{~V}$ points are indicated with the two vertical dashed lines. The symbols $\square$ and $\odot$ represent the 150 and $300 \mathrm{~V}$ points on the graph. 
Table 7-2. Parameters of saturation curves measured with ionization chambers (PR0-6C, NE2571, Holt and PEEC chamber) in $18 \mathrm{MV}$ pulsed photon beams at a dose rate of $\sim 400 \mathrm{cGy} / \mathrm{min}$.

\begin{tabular}{|c|c|c|c|c|c|c|}
\hline Chamber & $\begin{array}{c}2 \\
\text { Field size } \\
\left(\mathrm{cm}^{2}\right)\end{array}$ & $\begin{array}{c}I_{\text {sat }}(\mathrm{nA}) \\
\text { extrapolated } \\
\text { linear region }\end{array}$ & $\begin{array}{c}I_{\text {sat }}(\mathrm{nA}) \\
\text { "two-voltage" } \\
\text { technique } \\
\text { Eq. }(7-6)\end{array}$ & \begin{tabular}{|c|}
5 \\
Percent \\
difference \\
$100 \frac{(\operatorname{col}(4)-\operatorname{col}(3))}{\operatorname{col}(3)}$
\end{tabular} & $\lambda_{\mathrm{p}}(\mathrm{V} / \mathrm{nA})$ & $10^{5} \gamma\left(V^{-1}\right)$ \\
\hline & & $\pm 0.05 \%$ & $\pm 0.05 \%$ & $\pm 0.15 \%$ & & \\
\hline & $10 \times 10$ & 1.499 & 1.508 & 0.53 & $1.29 \pm 0.01$ & $1.48 \pm 0.05$ \\
\hline PR0-6C & $\begin{array}{l}10 \times 10 \\
\text { shielded }\end{array}$ & 1.495 & 1.505 & 0.67 & $1.30 \pm 0.01$ & $1.38 \pm 0.08$ \\
\hline & $30 \times 30$ & 1.627 & 1.713 & 5.3 & $1.30 \pm 0.02$ & $8.8 \pm 0.1$ \\
\hline NE2571 & $\begin{array}{l}10 \times 10 \\
30 \times 30\end{array}$ & $\begin{array}{l}1.457 \\
1.576\end{array}$ & $\begin{array}{l}1.459 \\
1.583\end{array}$ & $\begin{array}{l}0.14 \\
0.44\end{array}$ & $\begin{array}{l}2.06 \pm 0.02 \\
1.99 \pm 0.01\end{array}$ & $\begin{array}{l}0.45 \pm 0.05 \\
0.78 \pm 0.03\end{array}$ \\
\hline Holt & $\begin{array}{l}3 \times 3 \\
15 \times 15\end{array}$ & $\begin{array}{l}1.772 \\
2.392\end{array}$ & $\begin{array}{l}1.776 \\
2.413\end{array}$ & $\begin{array}{l}0.23 \\
0.88\end{array}$ & $\begin{array}{l}0.483 \pm 0.002 \\
0.418 \pm 0.005\end{array}$ & $\begin{array}{l}0.89 \pm 0.03 \\
2.80 \pm 0.2\end{array}$ \\
\hline PEEC & $\begin{array}{l}5 \times 5 \\
10 \times 10\end{array}$ & $\begin{array}{l}1.688 \\
1.947\end{array}$ & $\begin{array}{l}1.691 \\
1.952\end{array}$ & $\begin{array}{l}0.17 \\
0.25\end{array}$ & $\begin{array}{l}0.353 \pm 0.005 \\
0.359 \pm 0.007\end{array}$ & $\begin{array}{l}0.40 \pm 0.02 \\
0.64 \pm 0.03\end{array}$ \\
\hline
\end{tabular}

The saturation curves for the $10 \times 10 \mathrm{~cm}^{2}$ open field and for the field with "shielded stem" are essentially identical, except for the $I_{\text {sat }}$ current which is slightly lower for the "shielded stem" curve because of the lower radiation level in the shielded region of the beam. Both saturation curves are characterized by similar $\gamma$ values, suggesting that the high polarizing voltage non-linearity in the $1 / I$ vs. $1 / V$ plot for the $10 \times 10 \mathrm{~cm}^{2}$ fields cannot be attributed to extracameral effects. The $30 \times 30 \mathrm{~cm}^{2}$ saturation curve data, on the other hand, exhibit a considerably more pronounced non-linearity in comparison with the two $10 \times 10 \mathrm{~cm}^{2}$ curves, suggesting a strong field size dependence of the saturation data. It 
thus appears that the non-linearity in the saturation curves originates not only through charge multiplication in the chamber sensitive volume which predominates at small radiation fields but also through extracameral radiation-induced spurious leakage charges. The latter effect becomes more pronounced as the field size increases and irradiates more of the chamber stem, connector and cable.

The charge multiplication depends exponentially on $V$, while the radiation-induced leakage currents will be linearly dependent on $V$. Thus Eq. (7-7) will approximate the measured data well when the charge multiplication predominates or when $W$ is small, since for small $\gamma$ the leading edge of the exponential function $e^{\gamma V}$ is linear. However, when the radiation-induced leakage currents predominate, then Eq. (7-7) will fail to predict the measured data at very large chamber potentials. This is shown in Fig. 7-5(a) for the $30 \times 30 \mathrm{~cm}^{2}$ saturation data which, for relatively low polarizing voltages, may be approximated adequately with a $\gamma$ of $8.8 \times 10^{-5} \mathrm{~V}^{-1}$ in Eq. (7-7), yet the approximation fails to predict the saturation data at polarizing voltages above $150 \mathrm{~V}$.

Table 7-2 also shows that the parameters $\lambda_{p}$ are similar for the three saturation curves of Fig. 7-5(a). The product $\lambda_{p} I_{\text {sat }}$ is constant within a few percent which indicates, as expected, that $\Lambda_{p}$ depends only on chamber characteristics and on initial charge density per pulse but not on field size. The field size dependence of the parameter $\gamma$, on the other hand, suggests that the breakdown in $1 / I$ vs. $1 / V$ linearity results from combined effects of charge multiplication in the chamber sensitive volume and extracameral polarizing voltage-dependent radiation-induced leakage current.

To evaluate the influence of chamber design on the breakdown in $1 / I$ vs. $1 / V$ linearity, we also measured saturation curves with an NE2571 cylindrical Farmer-type chamber and a Holt parallel-plate chamber. The NE2571 chamber was embedded in a Solid Water ${ }^{\mathrm{TM}}$ phantom at a depth of $5 \mathrm{~cm}$ and exposed to an $18 \mathrm{MV}$ photon beam at $\sim 400 \mathrm{cGy} / \mathrm{min}$ with two open field sizes: $10 \times 10 \mathrm{~cm}^{2}$ and $30 \times 30 \mathrm{~cm}^{2}$. Saturation data for the two field sizes are shown in Fig. 7-5(b), again with the solid lines representing the linear fit to low polarizing voltage data and the dashed curves representing the fit of Eq. (7-7) to measured data in the polarizing voltage range from 20 to $700 \mathrm{~V}$. The parameters of the two saturation curves are listed in Table 7-2. 
As shown in Fig. 7-5(b), the NE2571 chamber also exhibits non-linearity in the $1 / I$ vs. $1 / V$ data; however, in comparison with the PR0-6C chamber, the breakdown in linearity is much less pronounced and much less field size dependent. For the NE2571 chamber the discrepancy between $I_{\text {sat }}$ obtained through a linear extrapolation of low polarizing voltage data and with the "two-voltage" technique amounts to only $0.14 \%$ for the $10 \times 10$ $\mathrm{cm}^{2}$ field and $0.44 \%$ for the $30 \times 30 \mathrm{~cm}^{2}$ field; considerably lower than corresponding results obtained for the PR0-6C chamber.

A similar study was carried out with a Holt parallel-plate chamber embedded at a depth of $3 \mathrm{~cm}$ in a polystyrene phantom and irradiated with two fields (3x3 and $15 \times 15 \mathrm{~cm}^{2}$ ) in an $18 \mathrm{MV}$ beam at $\sim 400 \mathrm{cGy} / \mathrm{min}$. The saturation curves for the two fields are shown in Fig. 7-6, with the solid line representing the linear fit to low polarizing voltage data and the dotted line representing the "two-voltage" technique. Parameters of the two saturation curves are listed in Table 7-2. Again, the breakdown in the $1 / I$ vs. $1 / V$ linearity is evident at polarizing voltages above $150 \mathrm{~V}$, as are the field size independence of $\lambda_{p} I_{\text {sat }}$ and field size dependence of $\gamma$. The $\gamma$ value for the $15 \times 15 \mathrm{~cm}^{2}$ field is about three times larger than that for the $3 \times 3 \mathrm{~cm}^{2}$ field, suggesting a large contribution of polarizing voltage-dependent radiation-induced leakage to the signal measured at high polarizing voltages.

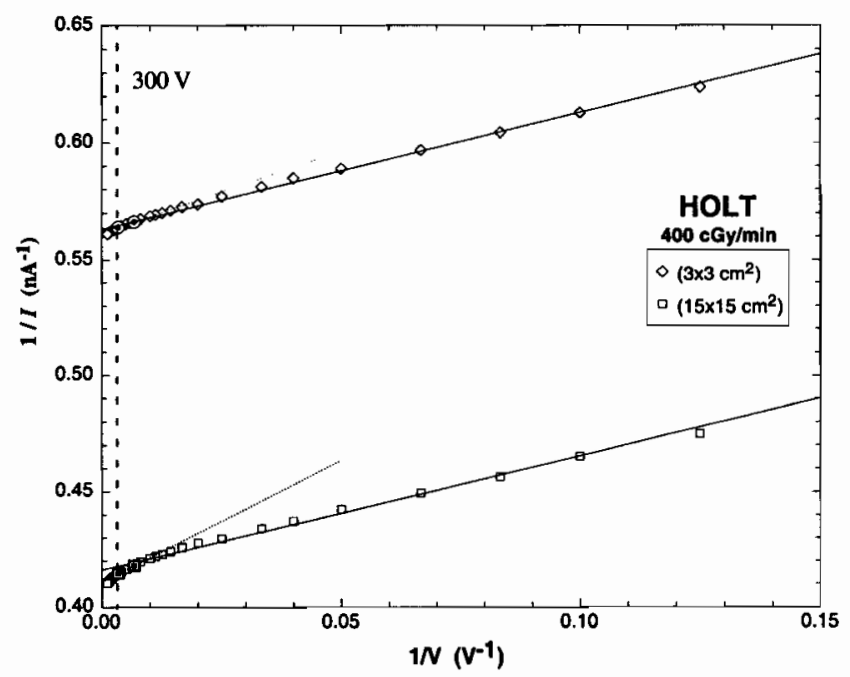

Figure 7-6. Saturation data measured with a Holt parallel plate ionization chamber in an $18 \mathrm{MV}$ pulsed photon beam at $\sim 400 \mathrm{cGy} / \mathrm{min}$ in the polarizing voltage region from 8 to $900 \mathrm{~V}$ for two fields: $3 \times 3 \mathrm{~cm}^{2}$ and $15 \times 15 \mathrm{~cm}^{2}$. The solid lines represent the linear fit to the linear portion of the data, the dashed lines the "two-voltage" technique. The symbols $\square$ and $\odot$ represent the 150 and $300 \mathrm{~V}$ points on the graph. 
Finally another similar study was carried out with the PEEC embedded at a depth of $10 \mathrm{~cm}$ in a Solid Water ${ }^{\mathrm{TM}}$ phantom and irradiated with two fields $\left(5 \times 5\right.$ and $\left.10 \times 10 \mathrm{~cm}^{2}\right)$ in an $18 \mathrm{MV}$ beam at $\sim 400 \mathrm{cGy} / \mathrm{min}$. The saturation curves for the two fields are shown in Fig. 7-7, with the solid line representing the fit of Eq. (7-7) to the data and the dotted line representing the "two-voltage" technique. Parameters of the two saturation curves are listed in Table 7-2. Again, the breakdown in the $1 / 1$ vs. $1 / V$ linearity is evident at polarizing voltages above $600 \mathrm{~V}$, as are the field size independence of $\lambda_{p} I_{\text {sat }}$ and field size dependence of $\gamma$. The $\gamma$ value for the $10 \times 10 \mathrm{~cm}^{2}$ field is about $50 \%$ larger than that for the $5 \times 5 \mathrm{~cm}^{2}$ field, suggesting a greater contribution of polarizing voltage-dependent radiation-induced leakage to the signal measured at high polarizing voltages.

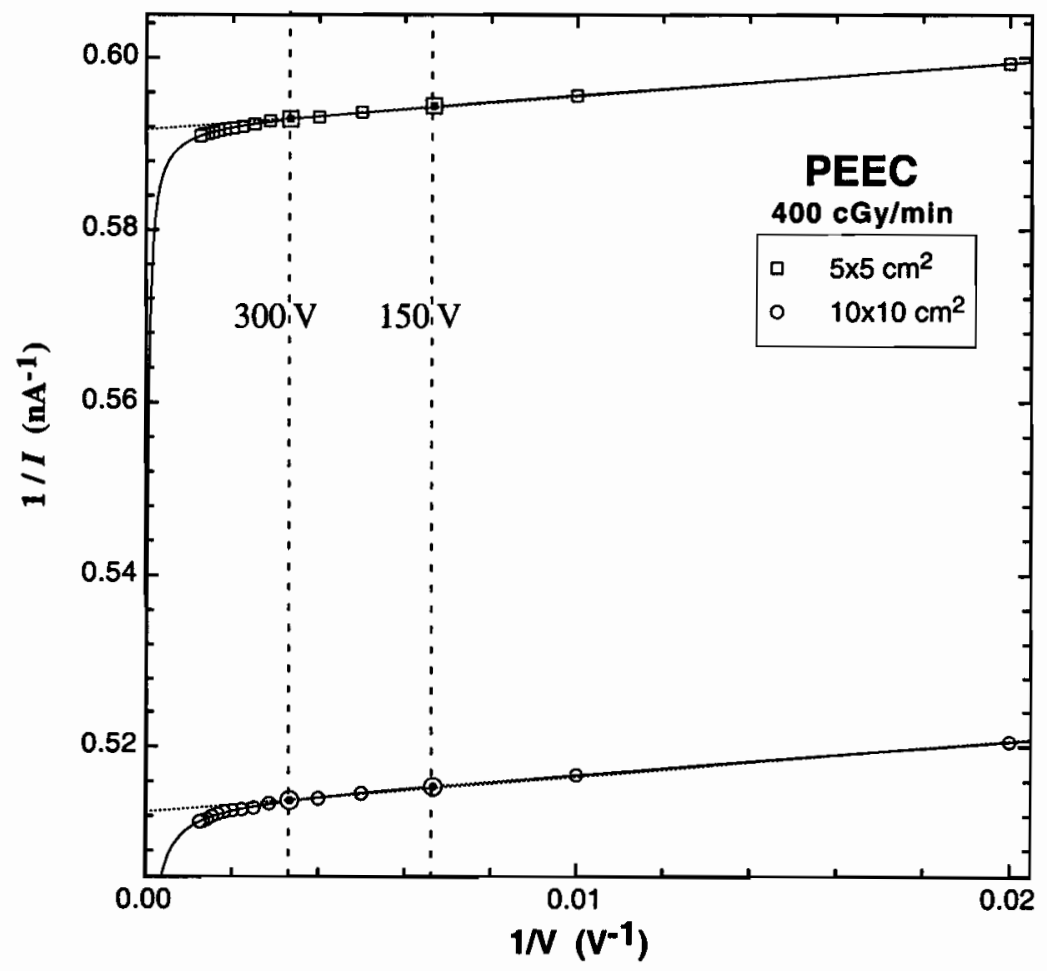

Figure 7-7. Saturation data measured with the Solid Water ${ }^{\text {TM }}$ PEEC parallel plate ionization chamber (with $2.5 \mathrm{~mm}$ electrode spacing) in an $18 \mathrm{MV}$ pulsed photon beam at $\sim 400 \mathrm{cGy} / \mathrm{min}$ in the polarizing voltage region from 50 to $800 \mathrm{~V}$ for two fields: $5 \times 5 \mathrm{~cm}^{2}$ and $10 \times 10 \mathrm{~cm}^{2}$. The dashed lines represent the linear fit to the linear portion of the data which is identical to the "two-voltage" technique result. The solid lines represent the fit to Eq. (7-7). The symbols $\square$ and $\odot$ represent the 150 and $300 \mathrm{~V}$ points on the graph. 
Figures 7-5 and 7-6 show results of saturation curve measurements in pulsed photon beams for three commercial ionization chambers. All chambers are suitable for use in absolute output measurements with existing radiation protocols and all exhibit a breakdown in the $1 / I$ vs. $1 / V$ linearity at standard chamber operating potentials. However, the severity of the breakdown depends on the type and design of the chamber as well as on the field size of the pulsed radiation beam.

The breakdown in the $1 / I$ and $1 / V$ relationship was even more pronounced for electron beams than for photon beams, as shown in Table I. However, our subsequent studies revealed that part of the breakdown in electron beams can be directly attributed to in-air measurements using an aluminium buildup cap with the chamber. This approach stabilized the chamber response to electron beams, but also caused a direct irradiation of a portion of the chamber stem. Since shielding of the stem decreased the $1 / I$ vs. $1 / V$ breakdown to the levels observed with the photon beams, we conclude that the breakdown in the near saturation region is of the same order of magnitude for both the photon and electron beams.

\section{Conclusions}

For ionization chambers exposed to pulsed megavoltage photon and electron beams, the linearity of the $1 / I$ and $1 / V$ plot, where $I$ and $V$ are the ionization chamber current and potential, respectively, breaks down in the polarizing voltage range where chambers are normally operated. The breakdown is caused by charge multiplication in the chamber sensitive volume and by leakage currents produced by radiation in the chamber stem, connector, and cable. The magnitude of this effect varies from one chamber to another and is also strongly field size dependent. The charge multiplication component depends exponentially on $V$, while the radiation-induced leakage component depends linearly on $V$. Both components are approximated with an exponential term $e^{\gamma V}$ in Boag's saturation curve equation. 
Depending on the particular ionization chamber model, the breakdown in the linearity between $1 / I$ and $1 / V$ may cause an overestimation of the radiation beam output by $\sim 0.7 \%$ for a $10 \times 10 \mathrm{~cm}^{2}$ field and by a few percent for large radiation fields when the standard "two-voltage" technique is used for determination of the saturation current. Therefore the "two-voltage" technique should be used with caution, and its reliability for a particular ionization chamber should be well established before the particular chamber is used in radiation dosimetry. Moreover, the choice of $300 \mathrm{~V}$ for the operating voltage might be excessive for certain ionization chambers, such as the Capintec PR0-6C. 


\section{References}

1 J. W. Boag, "Ionization chambers," in Radiation dosimetry, edited by F. H. Attix, W. C. Roesch and E. Tochilin (Academic Press, New York, 1966), Vol. 2, pp. 1-72.

2 J. W. Boag, "Ionization chambers," in The dosimetry of ionizing radiation, edited by K. R. Kase, B. E. Bjärngard and F. H. Attix (Academic Press, Orlando, 1985), Vol. 2, pp. 169-243.

3 C. E. Zankowski and E. B. Podgorsak, "Determination of saturation charge and collection efficiency for ionization chambers in continuous beams," Med. Phys. 25 (6), 908-915 (1998).

4 C. E. Zankowski, "Calibration of photon and electron beams with an extrapolation chamber," Ph. D. Thesis, McGill University, 1997.

5 C. L. Yang, D. W. Rogers, K. R. Shortt et al., "Ion recombination in ion chambers in continuous radiation," proceedings of the $45^{\text {th }}$ scientific meeting of the COMP, Sherbrooke, June 16-19, 23-25 (1999).

6 K. Derikum and M. Roos, "Measurement of saturation correction factors of thimble-type ionization chambers in pulsed photon beams," Phys. Med. Biol. 38 (6), 755-763 (1993).

7 J. W. Boag, "The saturation curve for ionization measurements in pulsed radiation beams," Brit. J. Radiol. 25, 649-650 (1952).

8 ICRU, “The dosimetry of pulsed radiation," ICRU Report 34, International Commission on Radiation Units and Measurements, Washington, D.C. (1982).

9 AAPM Task Group 21 of the Radiation Therapy Committee, "A protocol for the determination of absorbed dose from high-energy photon and electron beams," Med. Phys. 10, 741-771 (1983). 
10 R. J. Schulz, P. R. Almond, G. Kutcher et al., "Clarification of the AAPM Task Group 21 protocol," Med. Phys. 13 (5), $755-759$ (1986).

11 M. S. Weinhous and J. A. Meli, "Determining Pion, the correction factor for recombination losses in an ionization chamber," Med. Phys. 11 (6), 846-849 (1984).

12 J. A. Rawlinson, A. F. Bielajew, P. Munro et al., "Theoretical and experimental investigation of dose enhancement due to charge storage in electron-irradiated phantoms," Med. Phys. 11 (6), 814-821 (1984).

13 J. G. Holt, A. Buffa, D. J. Perry et al., "Absorbed dose measurements using parallel plate polystyrene ionization chambers in polystyrene phantoms," Int. J. Radiat. Oncol. Biol. Phys. 5 (11-12), 2031-2038. (1979).

14 J. W. Boag, E. Hochhauser, and O. A. Balk, "The Effect of Free-Electron Collection On the Recombination Correction to Ionization Measurements of Pulsed Radiation," Phys. Med. Biol. 41 (5), 885-897 (1996). 


\section{Chapter 8}

\section{Measurement of absorbed dose in bone-equivalent material}

\section{INTRODUCTION}

The phantom-embedded extrapolation chamber (PEEC) has many clinically relevant capabilities, one of them being the relative ease of measuring, in an absolute manner, the absorbed dose in tissue-equivalent materials. The modification of the chamber design, as described in Chapter 5, such that a given non-tissue-like material comprises the polarizing electrode window and the back of the collecting electrode, extended the capabilities of the PEEC to absolute dose determination in biological materials other than tissue. This chapter presents an analysis of factors which allow the use of the PEEC to measure dose directly in bone-equivalent material. It also gives results of our dose measurements in a bone-equivalent phantom.

The output calibration for a radiotherapy machine is generally measured as a dose rate, in cGy/min for radioisotope units and low energy $\mathrm{x}$-ray units and in cGy/MU for megavoltage linear accelerators. MU refers to "Monitor Unit", the basic unit of dose delivery in medical linacs. The dose rate is measured at a fixed depth in a water-equivalent phantom for a nominal field size (usually $10 \times 10 \mathrm{~cm}^{2}$ ) and a nominal source-phantom surface distance (usually $100 \mathrm{~cm}$ ). Dose rate at any other point in the treated volume is calculated using pre-measured physical parameters, such as percent depth dose and off-axis ratio, which relate the dose rate at the point of interest in the patient to the measured absolute dose rate at the reference point in a water-equivalent phantom. The ICRU ${ }^{1}$ recommended an overall accuracy in dose delivery to the target volume of $\pm 5 \%$, based on an analysis of dose response data and an evaluation of errors in dose delivery. This uncertainty in dose includes the treatment machine output calibration which should be precise to within $\pm 2 \%$ according 
to the $\mathrm{ICRU}^{2}$, the remaining $\pm 3 \%$ being uncertainties inherent to the measured physical parameters relating the dose rate at the reference point to the dose rate at arbitrary points in the treated volume.

Extrapolation chambers were first proposed for use in radiation detection by Failla $^{3}$ in 1937 and were subsequently used in measurement of surface doses in orthovoltage $^{3}$ and megavoltage ${ }^{4} \mathrm{x}$-ray beams and in dosimetry of $\beta$ rays ${ }^{5-7}$ and low-energy $\mathrm{x}$-rays. In 1955 , Genna and Laughlin ${ }^{8}$ proposed the use of extrapolation chambers in the measurement of radiotherapy machine output and calibrated a cobalt- 60 beam with a specially designed extrapolation chamber in a polystyrene phantom. Further measurements of megavoltage photon and electron beam outputs with an extrapolation chamber were carried out by Klevenhagen ${ }^{9}$ who built a Lucite-based PEEC and Zankowski and Podgorsak who used a Solid Water ${ }^{\mathrm{TM}}$ PEEC ${ }^{10-12}$. The use of a Solid Water ${ }^{\mathrm{TM}}$ PEEC allows a direct measurement of dose-to-Solid Water ${ }^{\mathrm{TM}}$, avoiding any corrections to the measured signal other than for charge losses within the chamber. Zankowski has shown that the uncalibrated PEEC can be used for radiation dosimetry with an accuracy similar to that obtained with calibrated ionization chambers used in conjunction with radiation dosimetry protocols ${ }^{13-15}$.

\section{Absorbed dose determination With the PEEC}

The PEEC design was described in detail in Chapter 5. In principle, determination of absolute dose at a given depth in a heterogeneous PEEC phantom is simple and based on first principles using the slope of the measured ionization as a function of the electrode separation (i.e., as a function of the sensitive air volume).

As described in Chapter 4, the Spencer-Attix air cavity relationship ${ }^{16}$ for determining the dose $D_{m e d}$ in phantom is as follows:

$$
D_{m e d}=\frac{Q}{m} \bar{W}_{\text {air }}(\bar{L} / \rho)_{\text {air }}^{\text {med }},
$$

where $Q$ is the charge collected under saturation conditions in the chamber sensitive air mass $m, \bar{W}_{a i r}$ is the mean energy required to produce an ion pair in air, and $(\bar{L} / \rho)_{\text {air }}^{\text {med }}$ is 
the ratio of restricted mass collisional stopping powers of the medium and air for the electron spectrum at the position of the air cavity. The sensitive air volume is assumed to satisfy the Bragg-Gray cavity condition which stipulates that the cavity is sufficiently small so that its presence does not perturb the charged particle field in the medium. Restricted mass stopping powers are averaged over the slowing down spectrum of all generations of electrons having kinetic energies in the range between a low energy limit $\Delta$ and the maximum electron energy. The value of $\Delta$ for the PEEC is taken as the minimum kinetic energy $(\approx 10 \mathrm{keV})$ required for an electron to be able to traverse a typical Bragg-Gray air cavity of 2 to $3 \mathrm{~mm}$ in width, as discussed in Chapter 6, Section IV.E.

For a small air cavity the ratio $Q / m$ of Eq. (8-1) is a constant as a function of the air mass $m$, so that it can be replaced by a relatively easily measurable quantity, $d Q / d m$. For a parallel-plate chamber, $m$ is given by $\rho z A$, where $A$ is the effective area of the collecting electrode, determined through techniques described in Chapter $5, z$ is the relative separation between the polarizing and measuring electrodes, and $\rho$ is the density of air ${ }^{17}$ in the cavity, corrected for temperature and pressure as follows:

$$
\rho=\left(\frac{P}{760}\right)\left(\frac{273.16}{273.16+\mathrm{T}}\right) \times 1.293 \times 10^{-3} \mathrm{~g} / \mathrm{cm}^{3}
$$

Equation (8-1) can now be rewritten as:

$$
D_{m e d}=\left(\frac{1}{\rho A}\right) \frac{d Q}{d z} \bar{W}_{a i r}(\bar{L} / \rho)_{\text {air }}^{\text {med }} .
$$

The derivative $d Q / d z$ in Eq. (8-3) is the slope of the $Q$ vs. $m$ relationship and is measured with the PEEC, as described in detail in Chapter 5.

\section{Precision of dose determination with the PEeC}

When using the PEEC, many parameters, such as the ionization gradient, air density, collecting electrode area, mean energy required to create an ion pair in air, and the 
mean mass restricted stopping power ratios, influence the uncertainty in the final measured dose value. This section presents a summary of the relative precision errors for all parameters in Eq. (8-3).

\section{III.A. Ionization gradient $d Q / d z$}

An absolute dose determination with the PEEC requires at least 8 ionization measurements to determine $d Q / d z$ and hence the dose by means of Eq. (8-3). Two ionization measurements are made at two different electrode spacings, for both polarities $(2 \times 2 \times 2=8)$ in order to determine the saturation charge $Q_{\text {sat }}$ through a two-voltage technique with the condition that measurements are made in the linear region of the saturation curve, as discussed in Chapter 7. This technique corrects for ionic recombination (initial and general recombination as well as diffusion) in the chamber sensitive volume; in dosimetry protocols this correction is normally accounted for by the parameter $p_{\text {ion }}$. The resulting $Q_{\text {sat }}$ values are used to calculate the slope $d Q / d z$. However, each ionization measurement is repeated at least three times or more, such that the standard error on each individual value is less than $0.1 \%$. This technique typically requires about 30 minutes over which changes in ambient temperature and pressure may occur. The observed variations in temperature and pressure were generally negligible, but sometimes they were on the order of $\sim 1{ }^{\circ} \mathrm{C}$ and $\sim 5 \mathrm{~mm} \mathrm{Hg}$, which could cause a $0.3 \%$ uncertainty in the determination of $Q_{\text {sat }}$. We therefore took the average of the temperature and pressure values at the beginning and at the end of the measurement procedure thereby minimizing the effects of any temperature and pressure change during the time of measurement.

We have shown in Chapter 5 that leakage contribution to the ionization measurements is negligible through the whole range of bias voltages under which the PEEC is operated. It was also shown in Chapter 5 that positioning errors caused by the non-linearity of the piston remote control system is less than $\pm 0.1 \%$. The overall uncertainty, obtained from the error on the slope $d Q / d z$ of the $d Q$ vs. $d z$ graph, is typically of the order of $\pm 0.1 \%$. 


\section{III.B. Air density $\rho$}

The air density depends on the air temperature and pressure through Eq. (8-2). Therefore, the temperature and pressure values were closely monitored when doing measurements with the PEEC to correct the air density values, as mentioned in Section III.A. We also assumed that the air humidity was constant during the time of measurement and therefore the error on the dose produced by the uncertainty in the air density value is deemed negligible.

\section{III.C. Electrode area $\boldsymbol{A}$}

An electrical method was used to determine the effective electrode area $A$, as described in Chapter 5. The same procedure was repeated many times for the same electrode over many weeks. The standard error on the calculated area was within $\pm 0.2 \%$ and therefore we assumed that this is the error introduced by this parameter. This uncertainty is caused by small imprecision in piston motion as well as by relative errors in the voltage provided by the power supply and possibly minute physical alterations in the collecting electrode edge which is etched onto the graphite layer to separate it from the guard electrode.

\section{III.D. Mean energy required to produce an ion pair in air, $\bar{W}_{\text {air }}$}

This value was assumed accurate for our work. Current clinical protocols also assume $\bar{W}_{\text {air }}$ to be accurate and therefore our dose determination is consistent with the current protocols. The currently accepted value is $\bar{W}_{\text {air }}=(33.97 \pm 0.05) \mathrm{J} / \mathrm{C}{ }^{18,19}$ for dry air. This quantity is mainly dependent on humidity which influences not only $\bar{W}_{\text {air }}$, but also the air density $\rho$ and the stopping power ratio $(\bar{L} / \rho)_{\text {air }}^{\text {med }}$, causing them to vary by anywhere up to $1 \%$. Historically, this caused some confusion in calibration protocols ${ }^{20}$ and at the present time a constant humidity correction factor $k_{h}=0.997$, applicable over a large range of humidities from $15 \%$ to $80 \%$, is used by the latest AAPM calibration protocol 
(TG-51) (Ref. \#15). It corrects the three quantities in the dose equation and is normally included in the basic output calibration of clinical ionization chambers. Therefore, for the PEEC we adopted the same approach and used a $-0.3 \%$ correction to the basic measurement readings after correcting for temperature and pressure.

\section{III.E. Mean restricted mass stopping power ratios $(\bar{L} / \rho)_{\text {air }}^{\text {med }}$}

There is agreement between various calculations at about the $0.1 \%$ level, if the same basic stopping power data are used. Current protocols are all based on stopping-power data from the ICRU Report 37 (Ref. \#19) which is based on the work of Berger and Seltzer at NIST ${ }^{21}$. The EGSnrc user code SPRRZnrc uses the beam phase spaces to calculate the mean mass restricted stopping power ratios (MRSPR) of all beams based on Berger and Seltzer formulation. The accuracy of our calculated mean restricted mass stopping power ratios (through SPRRZnrc) also depends on the accuracy at which we can determine the energy spectrum of the beam. Changing the initial energy of the electron beam in the BEAM simulation produces a different phase-space file. A $0.2 \mathrm{MeV}$ difference in the electron energy used for the accelerator simulation produces a variation of MRSPR less than $0.2 \%$.

The accuracy of the MRSPR also depends on the air cavity size of the PEEC. However, for a typical electron beam where one expects the most variation (almost constant with depth for a photon beam) we notice a negligible relative difference of less than $\pm 0.1 \%$, as presented in Section IV.B. Therefore, we assume the total uncertainty on the MRSPR to be $0.2 \%$.

\section{III.F. Overall precision of the PEEC}

The total uncertainty in the dose measurement with the PEEC is obtained through summing up the individual uncertainties in quadrature and then applying a square root to the sum. The individual components of the total uncertainty which were elucidated above are as follows: (1) determination of $d Q / d z$ including effects of air temperature and 
pressure $( \pm 0.1 \%)$; $(2)$ determination of collecting electrode area $( \pm 0.2 \%)$; $(3)$ mean mass restricted stopping power ratio $( \pm 0.2 \%)$; and they result in a total uncertainty of $\pm 0.3 \%$ in dose measurement in Solid Water ${ }^{\mathrm{TM}}$ material.

\section{ABSORBED DOSE DETERMINATION WITH THE HYBRID PEEC}

A measurement of the saturation charge $Q_{\text {sat }}$, corrected for recombination, as a function of electrode separation $z$ yields an accurate value of $d Q / d z$ and thus allows us to determine $D_{m e d}$ with the Solid Water ${ }^{\mathrm{TM}}$ PEEC. For measurement of dose to bone-equivalent material, the original PEEC design was modified by replacing the entrance-window and the material holding the collecting electrode by bone-equivalent material. The original assumption was that Eq. (8-3) could be applied directly to calculate dose-to-bone using mean mass restricted stopping power ratios bone to air.

In Table 8-1 we give results of our dose measurement in bone with the PEEC and a comparison with dose measurement in water for five clinical beams we studied: cobalt- 60 gamma rays (Theratron-780, AECL, Ottawa, ON); 6 and $18 \mathrm{MV}$ x rays (Clinac-2300 C/D, Varian, Palo Alto, CA); and 6 and $15 \mathrm{MeV}$ electrons (Clinac-18, Varian, Palo Alto, CA). Field size was $10 \times 10 \mathrm{~cm}^{2}, \operatorname{SSD} 100 \mathrm{~cm} .(\bar{L} / \rho)_{\text {air }}^{\text {bone }}$, given in column (2), was calculated for the respective points of interest. For photon beams the dose-to-bone $\left(D_{b o n e}\right)$ was measured with the hybrid PEEC at a depth of $6 \mathrm{~cm}$ and then corrected to $d_{\max }$ through appropriate percentage depth doses. For electron beams the bone dose was measured with the hybrid PEEC at $d_{\max }$

The measured doses to bone, given in column (4) of Table 8-1, are normalized to an exposure time of $1 \mathrm{~min}$ for cobalt beam and to $100 \mathrm{cGy}$ at depth of dose maximum in water for the linac photon and electron beams. The corresponding doses to water $\left(D_{\text {water }}\right)$ were measured with a calibrated cylindrical Farmer chamber (NE2571; Nuclear Enterprises, Beenham, Reading, England) in conjunction with the AAPM TG-51 dosimetry protocol $^{15}$ and are displayed in column (5) of Table 8-1. 
Table 8-1. Results of absolute calibration in bone-equivalent material carried out with a hybrid PEEC using graphite electrodes. Dose is calculated with Eq. (8-3), $\rho=1.293 \times 10^{-3} \mathrm{~g} / \mathrm{cm}^{3}, A=8.019 \mathrm{~cm}^{2}, \bar{W}_{\text {air }}=$ $33.97 \mathrm{~J} / \mathrm{C}$. $(\bar{L} / \rho)^{\text {bone }}$ is calculated with SPRRZnrc for each beam; $d Q / d z$ is measured at depth $d_{\max }$ for electron beams and at a depth of $6 \mathrm{~cm}$ for photon beams; ionization readings are corrected with the two voltage technique for ionic recombination and are also corrected for temperature, pressure and polarity effect. Dose (in cGy) is given at $d_{\max }$ (through appropriate percentage depth doses) for an exposure time of 1 minute for Co-60 (SSD $=80 \mathrm{~cm}$ ) and $100 \mathrm{cGy}$ in water for $10 \times 10 \mathrm{~cm}^{2}$ linac $(\mathrm{SSD}=100 \mathrm{~cm})$ beams. The graphite electrodes are $0.005 \mathrm{~cm}$ thick and a $2 \mathrm{~cm}$ bone thickness on the back of the collecting electrode is used.

\begin{tabular}{|c|c|c|c|c|c|c|c|}
\hline (1) & (2) & (3) & (4) & (5) & (6) & (7) & (8) \\
\hline $\begin{array}{l}\text { Beam } \\
\text { quality }\end{array}$ & $\begin{array}{c}(\bar{L} / \rho)_{\text {air }}^{\text {bone }} \\
\text { air depth } d_{\max } \text { ) }\end{array}$ & $\begin{array}{c}d Q / d z \\
\left.\text { (at depth } d_{\max }\right) \\
\left(10^{-5} \mathrm{C} / \mathrm{m}\right)\end{array}$ & $\begin{array}{c}\text { Dose bone } \\
D_{\text {bone }} \\
\\
\text { Eq. (8-3) } \\
\text { [PEEC] } \\
\text { (cGy) }\end{array}$ & $\begin{array}{c}\text { Dose Water } \\
D_{\text {water }}\left(d_{\max }\right) \\
\text { AAPM TG-51 } \\
\text { [Farmer] } \\
\text { (cGy) } \\
\quad \pm 1 \%\end{array}$ & $\begin{array}{l}\text { Measured } \\
\frac{D_{\text {bone }}}{D_{\text {water }}} \\
{\left[\frac{\operatorname{col}(4)}{\operatorname{col}(5)}\right]}\end{array}$ & $\begin{array}{c}\text { Monte Carlo } \\
\frac{D_{\text {bone }}}{D_{\text {water }}}\end{array}$ & $\begin{array}{c}\text { Difference } \\
{\left[\frac{\operatorname{col}(6)-\operatorname{col}(7)}{\operatorname{col}(7)}\right]} \\
(\%)\end{array}$ \\
\hline Co-60 & 0.994 & 3.039 & 98.97 & 109.2 & 0.906 & 0.949 & -4.5 \\
\hline $6 \mathrm{MV}$ & 0.984 & 2.944 & 94.9 & 100.0 & 0.949 & 0.976 & -2.8 \\
\hline $18 \mathrm{MV}$ & 0.959 & 3.195 & 100.4 & 100.0 & 1.004 & 1.042 & -3.7 \\
\hline $9 \mathrm{MeV}$ & 0.944 & 3.210 & 99.3 & 100.0 & 0.993 & 1.024 & -3.0 \\
\hline $15 \mathrm{MeV}$ & 0.933 & 3.206 & 98.0 & 100.0 & 0.980 & 0.993 & -1.3 \\
\hline
\end{tabular}

In column (6) of Table 8-1 we give the ratio $D_{\text {bone }} / D_{\text {water }}$ and note that the ratio deviates by a few percent from unity, with cobalt beam at 0.906 and the $18 \mathrm{MV}$ photon beam at 1.004 . These results seem reasonable, however, when we tried to confirm them with Monte Carlo calculations, we obtained considerably different results for $D_{\text {bone }} / D_{\text {water }}$, as evident from columns (7) and (8) of Table 8-1. For all beams the calculated data exceed the measured data. The discrepancy between measured results based on Eq. (8-3) and Monte Carlo results range from $-4.5 \%$ for the cobalt beam to $-1.3 \%$ for the $15 \mathrm{MeV}$ electron beam. These relatively large discrepancies observed between the measured and calculated $D_{\text {bone }} / D_{\text {water }}$ demonstrate that a direct application of Eq. (8-3) to signals measured with our hybrid PEEC does not lead directly to an absolute dose in bone.

To improve our understanding of this discrepancy we undertook a study of various parameters which might affect our dose-to-bone determination accuracy. These parameters were related to the chamber cavity as well as to the electrode configuration, and our study resulted in various correction factors which together correct the measured 
dose-to-bone and bring the result very close to Monte Carlo values, as shown in Section VI. below. In the following sections we discuss the techniques we used to evaluate the individual correction factors, accounting for: 1) scatter deficit, 2) air cavity perturbations, and 3) perturbations caused by electrodes.

The correction factors for scatter deficit, air cavity perturbations and electrode perturbations were calculated with Monte Carlo techniques; the last correction factor was also determined through relative air cavity dose measurements with various electrode combinations. These correction factors as well as the humidity correction factor $k_{h}$ are then taken into account in the dose equation through a multiplicative factor $C_{c h}$ which depends on the beam energy and the materials that constitute the electrodes. The inclusion of $C_{c h}$ into Eq. (8-3) gives the following relationship for the dose in bone-equivalent material:

$$
D_{m e d}=\left(\frac{1}{\rho A}\right) \frac{d Q}{d z} \bar{W}_{a i r}(\bar{L} / \rho){ }_{\text {air }}^{\text {bone }} C_{c h}
$$

An incorporation of appropriate $C_{c h}$ into the dose relationship results in dose-to-bone values that are very close to Monte Carlo-determined values allowing us to use the hybrid PEEC in measurement of absolute dose in heterogeneous materials.

\section{IV.A. Scatter correction}

The original PEEC was mainly composed of Solid Water ${ }^{\mathrm{TM}}$ (SW), a material that is often used as phantom material in calibration of megavoltage photon and electron beams. However, to extend measurement capabilities to heterogeneous materials and keep the costs reasonable only the entrance window and part of the back piston were replaced with the bone material. The construction of an entirely new chamber embedded in bone material would simply be too costly. Measurements made at a fixed depth, typically $6 \mathrm{~cm}$ for photon beams and at $d_{\text {max }}$ for electron beams, were initially assumed to be made completely in bone material. For this assumption to hold, all scatter produced by radiation within the hybrid chamber, made of SW with the bone material add-ons, must be equivalent to scatter 
that would be produced by a PEEC made entirely of bone material. The validity of this assumption could be verified experimentally by comparing the dose measured in our hybrid chamber, i.e., a chamber made of SW with some bone material components, to the dose measured in a chamber composed entirely of bone material. However, the latter option would require a costly building of a complete bone PEEC, and in addition, other parameters, such as the contribution from the electrodes, could alter the measurement results. We therefore rely on theoretical Monte Carlo calculations for an investigation of the scatter deficit resulting from the specific design of our hybrid chamber.

Monte Carlo calculations in bone material were carried out by modelling the two different geometries, i.e., a chamber fully composed of bone material and a SW chamber with bone material add-ons (entrance window and backing of collecting electrode). The geometry of the two setups is illustrated in Fig. 8-1. The chamber is modelled, as described in Chapter 6, Section V. For simulations intending to study the scatter effect from the particular geometry of the chamber, the model does not incorporate any electrodes to avoid effects of the electrodes on the measurement results. The schematics of Fig. 8-1(a) corresponds to our experimental hybrid chamber and that of Fig. 8-1(b) corresponds to the ideal homogeneous geometry, i.e., the PEEC chamber made entirely of bone-equivalent material.

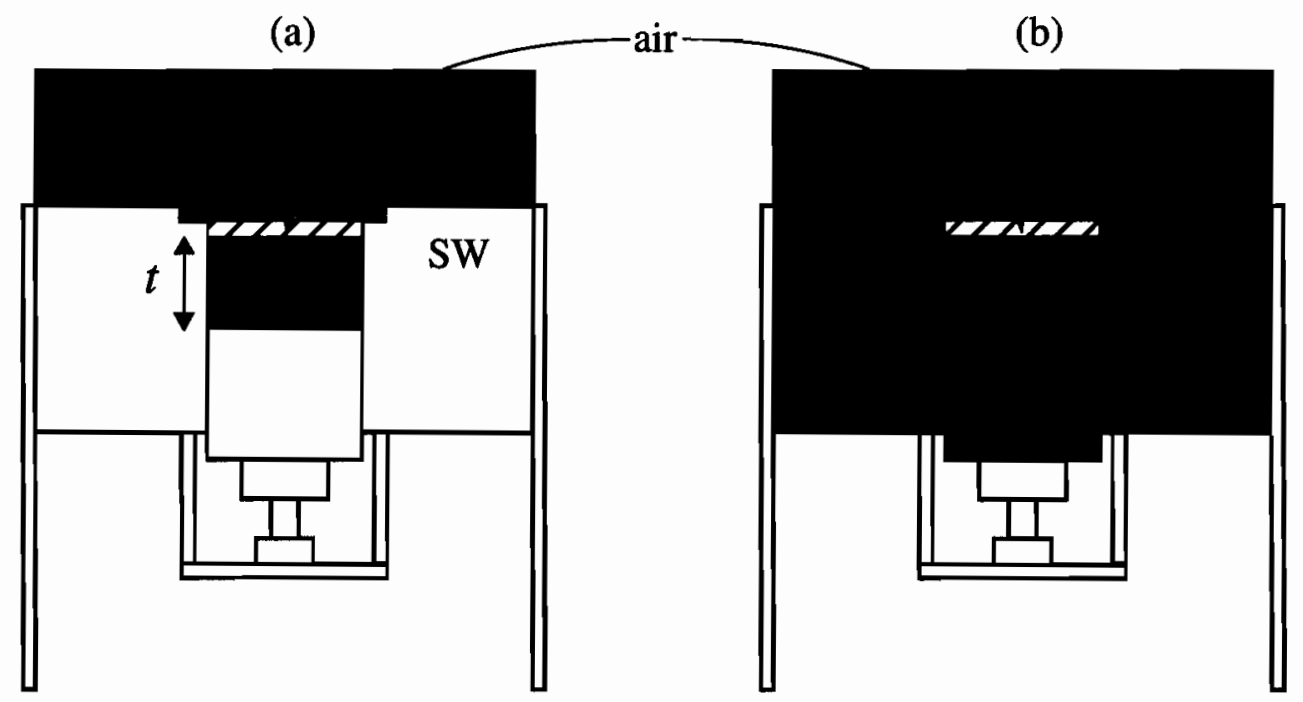

Figure 8-1. The simulation setup for scatter analysis of a bone-equivalent phantom: (a) hybrid chamber resulting from modification of the SW original chamber and (b) PEEC chamber built entirely from bone material. 
The dose to the air cavity was calculated for a typical air cavity thickness of 2 $\mathrm{mm}$ for a full bone PEEC and for our hybrid PEEC with three different thicknesses $t$ of bone material on the piston: $1 \mathrm{~cm}, 2 \mathrm{~cm}$, and $10 \mathrm{~cm}$. As far as experimental work is concerned, our dose measurements with the hybrid chamber were all carried out with a bone piston thickness of $2 \mathrm{~cm}$, as discussed in Chapter 5, Section I.B.

The calculated doses to the air cavity for the five clinical beams studied for the full bone PEEC and the hybrid PEEC are given in Table 8-2. The ratio $D(t) / D(\infty)$ which represents the dose to the air cavity for the hybrid chamber for a given bone thickness $t$ to the dose to the air cavity for the full bone PEEC $(t=\infty)$ is also given in order to show the effects of missing scatter in the hybrid chamber.

The results show that in comparison with a full bone ionization chamber, the hybrid design produces less scatter into the chamber sensitive volume, since the dose to the air cavity of the hybrid PEEC is $0.1 \%$ to $\sim 2 \%$ less than that for a full bone phantom, depending on beam energy but not on thickness of the bone layer in the hybrid chamber. We conclude that phantom backscatter is not an issue and that the lack of scatter is caused principally by changes in lateral scatter, since the dose values for thicknesses of 1,2 and $10 \mathrm{~cm}$ of bone material are all essentially identical within their error.

The amount of missing scatter for the hybrid design depends on beam quality; the lower the energy the larger the discrepancy between the dose-to-air cavity for bone-equivalent PEEC and that for the hybrid PEEC. For the actual $2 \mathrm{~cm}$ bone piston design of our experimental chamber, there is a $2 \%$ difference for the cobalt-60 gamma ray beam, a $1.5 \%$ difference for the $6 \mathrm{MV}$ photon beam, a $0.7 \%$ difference for the $18 \mathrm{MV}$ photon beam, a $1.1 \%$ difference for the $9 \mathrm{MeV}$ electron beam and a $0.7 \%$ difference for the $15 \mathrm{MeV}$ electron beam. These differences must be accounted for when determining the absolute dose in a bone phantom with our PEEC hybrid chamber, as discussed in section Section VI below. 
Table 8-2. Monte Carlo calculated ratios of dose-to-air in the chamber cavity for various thicknesses $t(1,2$, and $10 \mathrm{~cm})$ of bone material below the collecting electrode for the hybrid bone PEEC. The air cavity ( $2 \mathrm{~mm}$ thick) is at depth $d_{\max }$ for electron beams and at a depth of $6 \mathrm{~cm}$ for photon beams, field size $10 \times 10 \mathrm{~cm}^{2}$, SSD $80 \mathrm{~cm}$ for cobalt-60 beam and SSD $100 \mathrm{~cm}$ for linacs beams.

\begin{tabular}{|c|c|c|}
\hline $\begin{array}{l}\text { Beam } \\
\text { Quality }\end{array}$ & $\begin{array}{l}\text { Calculated dose* } \\
\text { (Gy) }\end{array}$ & $\begin{array}{l}\text { Calculated dose ratio } \\
\qquad \frac{D(t)}{D(\infty)}\end{array}$ \\
\hline & \multicolumn{2}{|c|}{ Full bone PEEC (bone thickness $t=\infty$ ) } \\
\hline Co-60 & $(2.995 \pm 0.013) \times 10^{-14}$ & 1.000 \\
\hline $6 \mathrm{MV}$ & $\left(2.315 \pm 0.011 \times 10^{-15}\right.$ & 1.000 \\
\hline $18 \mathrm{MV}$ & $(4.913 \pm 0.025) \times 10^{-15}$ & 1.000 \\
\hline $9 \mathrm{MeV}$ & $(1.1800 \pm 0.0025) \times 10^{-12}$ & 1.000 \\
\hline \multirow[t]{2}{*}{$15 \mathrm{MeV}$} & $(7.650 \pm 0.018) \times 10^{-13}$ & 1.000 \\
\hline & \multicolumn{2}{|c|}{ Bone thickness $t=1 \mathrm{~cm}$} \\
\hline Co-60 & $(2.917 \pm 0.012) \times 10^{-14}$ & $0.974 \pm 0.006$ \\
\hline $6 \mathrm{MV}$ & $(2.2676 \pm 0.0086) \times 10^{-15}$ & $0.979 \pm 0.006$ \\
\hline $18 \mathrm{MV}$ & $(4.910 \pm 0.027) \times 10^{-15}$ & $0.999 \pm 0.007$ \\
\hline $9 \mathrm{MeV}$ & $(1.167 \pm 0.021) \times 10^{-12}$ & $0.989 \pm 0.003$ \\
\hline \multirow[t]{2}{*}{$15 \mathrm{MeV}$} & $(7.562 \pm 0.017) \times 10^{-13}$ & $0.989 \pm 0.003$ \\
\hline & \multicolumn{2}{|c|}{ Bone thickness $t=2 \mathrm{~cm}$} \\
\hline Co-60 & $(2.936 \pm 0.009) \times 10^{-14}$ & $0.980 \pm 0.005$ \\
\hline $6 \mathrm{MV}$ & $(2.279 \pm 0.012) \times 10^{-15}$ & $0.985 \pm 0.007$ \\
\hline $18 \mathrm{MV}$ & $(4.880 \pm 0.019) \times 10^{-15}$ & $0.993 \pm 0.006$ \\
\hline $9 \mathrm{MeV}$ & $(1.167 \pm 0.002) \times 10^{-12}$ & $0.989 \pm 0.003$ \\
\hline \multirow[t]{2}{*}{$15 \mathrm{MeV}$} & $(7.599 \pm 0.020) \times 10^{-13}$ & $0.993 \pm 0.004$ \\
\hline & \multicolumn{2}{|c|}{ Bone thickness $t=10 \mathrm{~cm}$} \\
\hline Co-60 & $(2.956 \pm 0.012) \times 10^{-14}$ & $0.987 \pm 0.006$ \\
\hline $6 \mathrm{MV}$ & $(2.2767 \pm 0.0097) \times 10^{-15}$ & $0.983 \pm 0.006$ \\
\hline $18 \mathrm{MV}$ & $(4.892 \pm 0.023) \times 10^{-15}$ & $0.996 \pm 0.007$ \\
\hline $9 \mathrm{MeV}$ & $(1.1667 \pm 0.0019) \times 10^{-12}$ & $0.989 \pm 0.003$ \\
\hline $15 \mathrm{MeV}$ & $(7.579 \pm 0.018) \times 10^{-13}$ & $0.991 \pm 0.003$ \\
\hline
\end{tabular}

*air cavity dose per incident particle crossing the plane at SSD. 


\section{IV.B. Air-cavity perturbations}

In general, ionization chambers are not ideal Bragg-Gray cavities and, as discussed in Chapter 4, clinical protocols incorporate a variety of correction factors to correct for deviations from the Bragg-Gray cavity. The deviations are due to the shape of the cavity and its surrounding material causing a perturbation in the electron fluence. In addition, the actual point at which the cavity is measuring the dose is not defined uniquely. It is generally accepted that for large parallel-plate chambers the point of measurement is on the inside face of the front window of the chamber. For other types of chambers, such as cylindrical Farmer-type chambers, a gradient correction factor $p_{g r}$ is applied to the measured signal. This factor is normally included in the replacement correction factor, $p_{\text {repl }}$, which also includes a fluence correction factor $p_{f l}$ that corrects for the presence of the cavity in the phantom. The gradient correction factor $p_{g r}$ for a cylindrical chamber is a function of the radius of the cavity and the local ionization gradient. For our PEEC, which is a parallel-plate chamber, we assumed that the measurement point was on the interior surface of the polarizing electrode.

Previous work with the original PEEC assumed that no cavity or wall corrections were required. Absolute dose measurements carried out with the Solid Water ${ }^{\mathrm{TM}}$ PEEC agreed very well ${ }^{10-12}$ with doses determined through standard calibration protocols ${ }^{13,22}$. However, as discussed in the beginning of this section, for determination of dose-to-bone there is a considerable discrepancy between the measured dose (Eq. (8-3)) and the predicted dose calculated with Monte Carlo techniques, especially for low energy photon beams.

Zankowski $^{11}$ determined experimentally that the PEEC cavity provided a good Bragg-Gray cavity approximation when the electrode spacing was between 2 to $3 \mathrm{~mm}$. Therefore, the PEEC air cavity that is used for our dose measurement has a variable thickness in the range from 2 to $3 \mathrm{~mm}$. For this range of electrode spacings it is also possible to simulate the dose given to the air cavity and compare the calculated value with that obtained for the cavity completely filled with the material of interest.

The ratio of dose to the air cavity to dose to an equivalent Solid Water ${ }^{\mathrm{TM}}$ voxel, centered at the polarizing electrode position (depth of measurement), was calculated as a 
function of electrode separation, and the results are shown in Fig. 8-2 for a $9 \mathrm{MeV}$ electron beam. For the range of electrode separations used in our measurements ( 2 to $3 \mathrm{~mm}$ ) the dose ratio is essentially constant and agrees well with the mean restricted stopping power ratio with a cut-off $\Delta$ of $10 \mathrm{keV}\left[\left.(\bar{L} / \rho)_{\text {air }}^{\text {sw }}\right|_{\Delta=10 \mathrm{keV}}=0.965\right]$. As expected, for larger (above $3 \mathrm{~mm}$ ) and for smaller (below $1 \mathrm{~mm}$ ) cavity gaps, the dose ratio is no longer constant. A similar behavior was observed with other beam energies for Solid Water ${ }^{\mathrm{TM}}$ and bone PEEC. Based on these results we used our hybrid PEEC also with gaps in the range between 1 and $3 \mathrm{~mm}$.

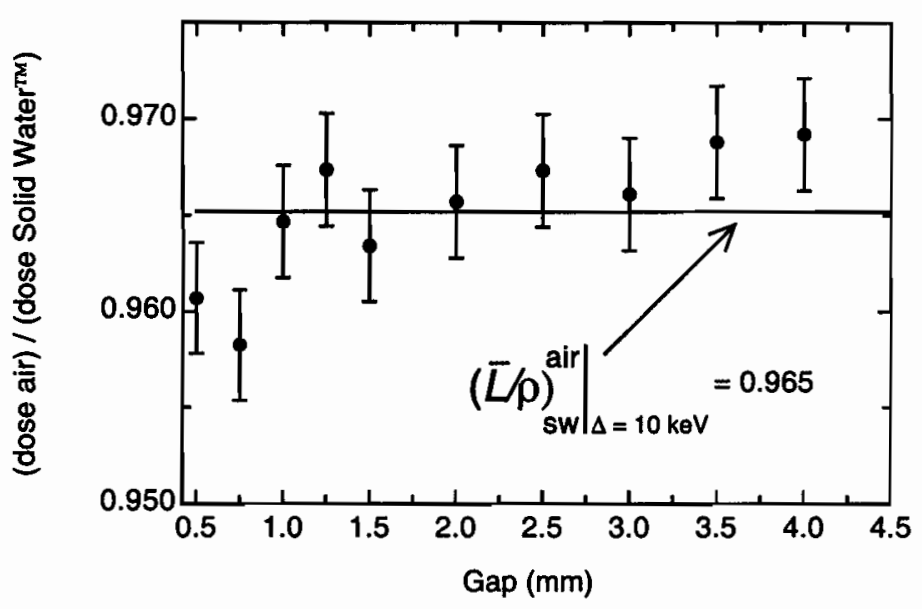

Figure 8-2. Air cavity perturbation factor for $9 \mathrm{MeV}$ for the Solid Water ${ }^{\mathrm{TM}}$ PEEC.

\section{IV.C. Correction for chamber wall}

In ionization chamber dosimetry protocols, there is also a correction for the presence of a cavity wall of a different material than the material of the phantom. The correction factor is called $p_{\text {wall }}$ and its formulation is given in Eq. (4-47). In electron beams $p_{\text {wall }}$ has traditionally been assumed to be 1.00 . To model the effect of the wall material on the electron spectrum in the cavity, Nahum has developed a theory ${ }^{23}$ that agrees qualitatively with experimental data. This model shows that the wall effect should be less than $1 \%$. However, more recently, Klevenhagen ${ }^{24,25}$ and $\mathrm{Hunt}^{26}$ have demonstrated for parallel-plate chambers that backscatter radiation coming into the cavity depends on the material behind the air cavity. This backscatter radiation produces a non-negligible change in the ionization 
measurement. Preliminary Monte Carlo calculations of this effect were obtained by $\mathrm{Ma}^{27}$ for commercial parallel-plate chambers, showing an effect on the order of 1 to $2 \%$.

Since our chamber is embedded into the measurement material, either bone for the hybrid chamber or Solid Water ${ }^{\mathrm{TM}}$ for the original chamber, no wall correction factor is required for absolute dose determination with the two extrapolation chambers.

\section{IV.D. Correction for electrode perturbation}

The original PEEC graphite electrodes have a different atomic number and density $\left(Z=6\right.$ for graphite, $\left.\rho=1.7 \mathrm{~g} / \mathrm{cm}^{3}\right)$ than the bone-equivalent material $\left(Z_{e f f}=13.927\right.$, $\rho=1.84 \mathrm{~g} / \mathrm{cm}^{3}$ ). Therefore, their presence in the chamber is likely to affect the electron fluence in the chamber cavity. We used Monte Carlo techniques to evaluate the extent to which the composition of the collecting and polarizing electrodes affect the dose to the air cavity of the chamber.

\section{IV.D.1 Evaluation of graphite electrodes for the Solid Water ${ }^{\text {TM }}$ PEEC}

Zankowski $^{11}$ in his work assumed that graphite electrodes do not have any adverse effects on absolute dose measurement with the Solid Water ${ }^{\text {TM }}$ PEEC and confirmed the assumption through a comparison of doses determined with the SW PEEC with those determined with calibrated Farmer chambers in conjunction with dosimetry protocols. We confirmed the validity of this assumption using Monte Carlo techniques, discussed in Chapter 6; a direct experimental verification is not possible, since the charges in the chamber cannot be collected without suitable electrodes. The SW PEEC model, shown in Fig. 6-20, was used with DOSRZnrc to calculate the dose to the air cavity without any electrodes and the dose to the air cavity with graphite electrodes, both collecting and polarizing electrodes having a thickness of $0.005 \mathrm{~cm}$. Results for the five clinical beams that we studied are shown in Table 8-3. 
Table 8-3. Monte Carlo calculated ratios of air cavity dose for SW PEEC with and without $0.005 \mathrm{~cm}$ thick graphite electrodes. The air cavity ( $2 \mathrm{~mm}$ thick) is at depth $d_{\max }$ for electron beams and at a depth of $6 \mathrm{~cm}$ for photon beams, field size $10 \times 10 \mathrm{~cm}^{2}$, SSD $80 \mathrm{~cm}$ for cobalt-60 beam and SSD $100 \mathrm{~cm}$ for linacs beams.

\begin{tabular}{|c|c|c|c|}
\hline \multirow[t]{2}{*}{ Beam Quality } & \multicolumn{2}{|c|}{$\begin{array}{l}\text { Calculated dose* } \\
\text { (Gy) }\end{array}$} & \multirow[t]{2}{*}{$\begin{array}{l}\text { Calculated dose ratio } \\
\qquad\left(\frac{\text { graphite }}{\text { no graphite }}\right)\end{array}$} \\
\hline & graphite & no graphite & \\
\hline $\mathrm{Co}-60$ & $(3.132 \pm 0.013) \times 10^{-14}$ & $(3.149 \pm 0.013) \times 10^{-14}$ & $0.995 \pm 0.006$ \\
\hline $6 \mathrm{MV}$ & $(2.374 \pm 0.020) \times 10^{-15}$ & $(2.353 \pm 0.018) \times 10^{-15}$ & $1.009 \pm 0.012$ \\
\hline $18 \mathrm{MV}$ & $(4.669 \pm 0.010) \times 10^{-15}$ & $(4.656 \pm 0.009) \times 10^{-15}$ & $1.003 \pm 0.003$ \\
\hline $9 \mathrm{MeV}$ & $(9.569 \pm 0.020) \times 10^{-13}$ & $(9.540 \pm 0.014) \times 10^{-13}$ & $1.003 \pm 0.003$ \\
\hline $15 \mathrm{MeV}$ & $(6.750 \pm 0.017) \times 10^{-13}$ & $(6.748 \pm 0.018) \times 10^{-13}$ & $1.000 \pm 0.004$ \\
\hline
\end{tabular}

*air cavity dose per incident particle crossing the plane at SSD.

The results indicate that the choice of graphite electrodes is suitable for the SW PEEC. All calculated ratios of dose with graphite to dose without graphite are equal to 1 within their respective errors. The lower energy beams (cobalt-60 and $6 \mathrm{MV}$ x-rays) show the largest effect, the dose being $0.5 \%$ lower and $0.9 \%$ higher, respectively, for the two beams. A small correction factor, less than $1 \%$, would thus be indicated for the lower energy beams, however, better statistics would be required on our Monte Carlo calculations to estimate the exact corrections. Even if a 1\% correction had to be applied for the lower energy beams, the relative error on the absolute dose measurements with the SW PEEC is still less than the clinically acceptable $\pm 2 \%$, considering a $\pm 0.3 \%$ uncertainty introduced by the basic PEEC parameters, summarized in Section III.F, and a less than $\pm 1 \%$ from the fluence perturbation caused by the graphite electrodes. Therefore, it is reasonable to assume that the SW PEEC with graphite electrodes can be used without any cavity and wall correction factors in determination of absolute dose in Solid Water ${ }^{\mathrm{TM}}$. 


\section{IV.D.2 Effect of electrode material on the hybrid PEEC}

Monte Carlo calculations were also carried out to investigate the effect of the polarizing and collecting electrode materials on dose determination with our hybrid PEEC. The following electrode materials were studied: graphite, aluminum, steel, and brass; electrodes made of carbon and aluminum were $0.005 \mathrm{~cm}$ thick, electrodes made of steel and brass were $0.0025 \mathrm{~cm}$ thick. As shown in Tables 8-4 and 8-5, the dose-to-air cavity was calculated for various polarizing/collecting electrode combinations and the results were compared with dose-to-air cavity calculated for no electrodes present.

The ratio (dose with electrodes)/(dose with no electrodes) deviates from unity with varying degree, depending on beam type and energy as well as combination of electrode materials. The graphite/graphite electrode combination used experimentally in our hybrid PEEC shows a fairly large discrepancy from 1 (up to 5\%), indicating that electrode materials, despite being very thin, have a considerable effect on bone dose measurements.

Of the electrode materials studied the combination of graphite polarizing electrode and aluminum collecting electrode gave the best results which deviated from no electrode data by less than $1 \%$ for all beams studied. The collecting electrode affects the measured dose more than the polarizing electrode, most likely as the result of backscattering into the cavity ${ }^{24-27}$.

Absolute dose-to-bone measurements with PEEC were carried out with graphite polarizing and graphite collecting electrodes. The effect of the graphite electrodes was accounted for by including the corresponding ratios (graphite/graphite) from Tables 8-4 and 8-5 into the total dose correction factor $C_{c h}$. 
Table 8-4. Percent difference between dose to air cavity with no electrode and with specific electrode combinations for photon beams. Graphite and aluminum electrodes are $0.005 \mathrm{~cm}$ thick, steel and brass electrodes are $0.0025 \mathrm{~cm}$ thick, for a $2 \mathrm{~cm}$ bone piston material on the back of the collecting electrode. The data were calculated with Monte Carlo techniques. The air cavity $(2 \mathrm{~mm}$ thick) is at depth $6 \mathrm{~cm}$, field size $10 \times 10 \mathrm{~cm}^{2}$, SSD $80 \mathrm{~cm}$ for cobalt-60 beam and SSD $100 \mathrm{~cm}$ for linacs beams.

\begin{tabular}{|c|c|c|c|c|}
\hline $\begin{array}{c}\text { Beam } \\
\text { quality }\end{array}$ & $\begin{array}{c}\text { Material } \\
\text { (polarizing/collecting) }\end{array}$ & $\begin{array}{l}\text { Calculated dose * } \\
\text { (Gy) }\end{array}$ & $\begin{array}{l}\text { Calculated dose ratio } \\
\left(\frac{\text { dose with electrodes }}{\text { dose without electrodes }}\right)\end{array}$ & $\begin{array}{l}\% \text { difference from } \\
\text { "no electrodes" } \\
\text { (calculated dose ratio) - } 1 \text { ) }\end{array}$ \\
\hline \multirow[t]{7}{*}{ Co-60 } & no electrode & $(2.936 \pm 0.009) \times 10^{-14}$ & $1.000 \pm 0.005$ & 0.0 \\
\hline & graphite/graphite & $(2.800 \pm 0.012) \times 10^{-14}$ & $0.954 \pm 0.005$ & -4.6 \\
\hline & graphite/Al & $(2.958 \pm 0.013) \times 10^{-14}$ & $1.007 \pm 0.005$ & 0.7 \\
\hline & Al/graphite & $(2.834 \pm 0.012) \times 10^{-14}$ & $0.965 \pm 0.005$ & -3.5 \\
\hline & $\mathrm{Al} / \mathrm{Al}$ & $(3.041 \pm 0.014) \times 10^{-14}$ & $1.036 \pm 0.006$ & 3.6 \\
\hline & steel/graphite & $(3.007 \pm 0.012) \times 10^{-14}$ & $1.024 \pm 0.005$ & 2.4 \\
\hline & brass/graphite & $(3.110 \pm 0.012) \times 10^{-14}$ & $1.059 \pm 0.005$ & 5.9 \\
\hline \multirow[t]{7}{*}{$6 \mathrm{MV}$} & no electrode & $(2.279 \pm 0.012) \times 10^{-15}$ & $1.000 \pm 0.007$ & 0.0 \\
\hline & graphite/graphite & $(2.191 \pm 0.013) \times 10^{-15}$ & $0.961 \pm 0.007$ & -3.88 \\
\hline & graphite/Al & $(2.293 \pm 0.015) \times 10^{-15}$ & $1.006 \pm 0.008$ & 0.61 \\
\hline & $\mathrm{Al} /$ graphite & $(2.224 \pm 0.015) \times 10^{-15}$ & $0.976 \pm 0.008$ & -2.41 \\
\hline & $\mathrm{Al} / \mathrm{Al}$ & $(2.343 \pm 0.018) \times 10^{-15}$ & $1.028 \pm 0.010$ & 2.78 \\
\hline & steel/graphite & $(2.31 \pm 0.01) \times 10^{-15}$ & $1.013 \pm 0.010$ & 1.3 \\
\hline & brass/graphite & $(2.37 \pm 0.01) \times 10^{-15}$ & $1.039 \pm 0.010$ & 3.9 \\
\hline \multirow[t]{7}{*}{$18 \mathrm{MV}$} & no electrode & $(4.881 \pm 0.019) \times 10^{-15}$ & $1.000 \pm 0.006$ & 0.0 \\
\hline & graphite/graphite & $(4.773 \pm 0.014) \times 10^{-15}$ & $0.977 \pm 0.004$ & -2.21 \\
\hline & graphite/Al & $(4.853 \pm 0.020) \times 10^{-15}$ & $0.994 \pm 0.006$ & -0.59 \\
\hline & $\mathrm{Al} /$ graphite & $(4.845 \pm 0.019) \times 10^{-15}$ & $0.993 \pm 0.006$ & -0.74 \\
\hline & $\mathrm{Al} / \mathrm{Al}$ & $(4.988 \pm 0.021) \times 10^{-15}$ & $1.022 \pm 0.006$ & 2.18 \\
\hline & steel/graphite & $(4.99 \pm 0.01) \times 10^{-15}$ & $1.023 \pm 0.005$ & 2.3 \\
\hline & brass/graphite & $(5.02 \pm 0.02) \times 10^{-15}$ & $1.029 \pm 0.006$ & 2.9 \\
\hline
\end{tabular}

*air cavity dose per incident particle crossing the plane at SSD. 
Table 8-5. Percent difference between dose to air cavity with no electrode and with specific electrode combinations for electron beams. Graphite and aluminum electrodes are $0.005 \mathrm{~cm}$ thick, steel and brass electrodes are $0.0025 \mathrm{~cm}$ thick, for a $2 \mathrm{~cm}$ bone piston material on the back of the collecting electrode. The data were calculated with Monte Carlo techniques. The air cavity ( $2 \mathrm{~mm}$ thick) is at depth $d_{\text {max }}$, field size $10 \times 10 \mathrm{~cm}^{2}$, and SSD $100 \mathrm{~cm}$.

\begin{tabular}{|c|c|c|c|c|}
\hline $\begin{array}{l}\text { Beam } \\
\text { quality }\end{array}$ & $\begin{array}{c}\text { Material } \\
\text { (polarizing/collecting) }\end{array}$ & $\begin{array}{l}\text { Calculated dose* } \\
\text { (Gy) }\end{array}$ & $\begin{array}{l}\text { Calculated dose ratio } \\
\left(\frac{\text { dose with electrodes }}{\text { dose without electrodes }}\right)\end{array}$ & $\begin{array}{l}\text { \% difference from } \\
\text { "no electrodes" } \\
\text { ((calculated dose ratio) - } \\
\text { 1) }\end{array}$ \\
\hline \multirow[t]{7}{*}{$9 \mathrm{MeV}$} & no electrode & $(1.167 \pm 0.002) \times 10^{-12}$ & $1.000 \pm 0.002$ & $\overline{0.00}$ \\
\hline & graphite/graphite & $(1.153 \pm 0.002) \times 10^{-12}$ & $0.988 \pm 0.002$ & -1.2 \\
\hline & graphite/Al & $(1.1720 \pm 0.0018) \times 10^{-12}$ & $1.005 \pm 0.002$ & 0.5 \\
\hline & Al/graphite & $(1.1610 \pm 0.0022) \times 10^{-12}$ & $0.995 \pm 0.003$ & -0.5 \\
\hline & $\mathrm{Al} / \mathrm{Al}$ & $(1.183 \pm 0.0024) \times 10^{-12}$ & $1.014 \pm 0.003$ & 1.4 \\
\hline & steel/graphite & $(1.178 \pm 0.002) \times 10^{-12}$ & $1.009 \pm 0.002$ & 0.9 \\
\hline & brass/graphite & $(1.184 \pm 0.002) \times 10^{-12}$ & $1.015 \pm 0.002$ & 1.5 \\
\hline \multirow[t]{7}{*}{$15 \mathrm{MeV}$} & no electrode & $(7.599 \pm 0.020) \times 10^{-13}$ & $1.000 \pm 0.004$ & 0.00 \\
\hline & graphite/graphite & $(7.506 \pm 0.018) \times 10^{-13}$ & $0.988 \pm 0.004$ & -1.2 \\
\hline & graphite/Al & $(7.619 \pm 0.020) \times 10^{-13}$ & $1.003 \pm 0.004$ & 0.3 \\
\hline & $\mathrm{Al} /$ graphite & $(7.543 \pm 0.022) \times 10^{-13}$ & $0.993 \pm 0.004$ & -0.7 \\
\hline & $\mathrm{Al} / \mathrm{Al}$ & $(7.695 \pm 0.018) \times 10^{-13}$ & $1.013 \pm 0.004$ & 1.3 \\
\hline & steel/graphite & $(7.62 \pm 0.02) \times 10^{-13}$ & $1.003 \pm 0.004$ & 0.3 \\
\hline & brass/graphite & $(7.67 \pm 0.02) \times 10^{-13}$ & $1.009 \pm 0.004$ & 0.9 \\
\hline
\end{tabular}

*air cavity dose per incident particle crossing the plane at SSD.

\section{IV.D.3 Experimental verification of electrode material effect}

Calculated data presented in Tables 8-4 and 8-5 show that electrode material has an appreciable effect on the dose received by the PEEC air cavity. To confirm this finding experimentally, we carried out a study of dose measurement with our hybrid PEEC using three different materials for the polarizing electrode (graphite, steel, and brass) in conjunction with a graphite measuring electrode. 
Results of the dose measurements are presented and compared to calculated values in Table 8-6 for 6 MV and $18 \mathrm{MV}$ x-ray beams and $9 \mathrm{MeV}$ and $15 \mathrm{MeV}$ electron beams. The signal produced by the air cavity is obviously affected by electrode material as shown in column (6) of Table 8-6, especially so for the low energy photon beams and brass polarizing electrode. Since the measured data of column (6) match the calculated data of column (5) within $2 \%$, we conclude that the Monte Carlo calculations summarized in Tables 8-4 and 8-5 have been experimentally confirmed.

Table 8-6. Comparison of dose-to-air ratios calculated with Monte Carlo and measured with bone PEEC for various electrode materials. The air cavity dose is given at depth $d_{\max }$ for electron beams and at a depth of 6 $\mathrm{cm}$ for photon beams, for $100 \mathrm{MU}$, field size $10 \times 10 \mathrm{~cm}^{2}$, SSD $100 \mathrm{~cm}$. Ratios are normalized to dose to air for the graphite/graphite dose. The air cavity thickness is varied from 2 to $3 \mathrm{~mm}$ for experimental data and is fixed at $2 \mathrm{~mm}$ for calculated data.

\begin{tabular}{|c|c|c|c|c|c|}
\hline (1) & (2) & (3) & (4) & (5) & (6) \\
\hline $\begin{array}{c}\text { Beam } \\
\text { quality }\end{array}$ & $\begin{array}{c}\text { Materials } \\
\text { (polarizing/collecting) }\end{array}$ & $\begin{array}{l}\text { Calculated dose* } \\
\text { (Gy) }\end{array}$ & $\begin{array}{l}\text { Measured dose } \\
\text { (Eq. (8-3)) } \\
\text { [PEEC] } \\
\text { cGy }\end{array}$ & $\begin{array}{c}\begin{array}{c}\text { Calculated } \\
\text { dose ratio }\end{array} \\
\left(\frac{D_{\text {polarizing/collecting }}}{D_{\text {graphite/graphite }}}\right)\end{array}$ & $\left(\begin{array}{c}\begin{array}{c}\text { Measured } \\
\text { dose ratio }\end{array} \\
\left(\frac{D_{\text {polarizing/collecting }}}{D_{\text {graphite/graphite }}}\right)\end{array}\right.$ \\
\hline $6 \mathrm{MV}$ & $\begin{array}{c}\text { graphite/graphite } \\
\text { steel/graphite } \\
\text { brass/graphite }\end{array}$ & $\begin{array}{l}2.191 \pm 0.013 \times 10^{-15} \\
2.309 \pm 0.018 \times 10^{-15} \\
2.367 \pm 0.014 \times 10^{-15}\end{array}$ & $\begin{array}{l}68.13 \pm 0.34 \\
71.84 \pm 0.36 \\
72.46 \pm 0.36\end{array}$ & $\begin{array}{l}1.000 \pm 0.006 \\
1.055 \pm 0.007 \\
1.082 \pm 0.007\end{array}$ & $\begin{array}{l}1.000 \pm 0.007 \\
1.054 \pm 0.007 \\
1.064 \pm 0.008\end{array}$ \\
\hline $18 \mathrm{MV}$ & $\begin{array}{c}\text { graphite/graphite } \\
\text { steel/graphite } \\
\text { brass/graphite }\end{array}$ & $\begin{array}{l}4.773 \pm 0.014 \times 10^{-15} \\
4.987 \pm 0.016 \times 10^{-15} \\
5.019 \pm 0.019 \times 10^{-15}\end{array}$ & $\begin{array}{l}83.56 \pm 0.42 \\
86.47 \pm 0.43 \\
87.08 \pm 0.44\end{array}$ & $\begin{array}{l}1.000 \pm 0.003 \\
1.046 \pm 0.003 \\
1.052 \pm 0.005\end{array}$ & $\begin{array}{l}1.000 \pm 0.007 \\
1.035 \pm 0.007 \\
1.042 \pm 0.007\end{array}$ \\
\hline $9 \mathrm{MeV}$ & $\begin{array}{c}\text { graphite/graphite } \\
\text { steel/graphite } \\
\text { brass/graphite }\end{array}$ & $\begin{array}{l}1.153 \pm 0.002 \times 10^{-12} \\
1.178 \pm 0.002 \times 10^{-12} \\
1.184 \pm 0.002 \times 10^{-12}\end{array}$ & $\begin{array}{l}108.78 \pm 0.54 \\
110.17 \pm 0.55 \\
111.21 \pm 0.56\end{array}$ & $\begin{array}{l}1.000 \pm 0.002 \\
1.022 \pm 0.002 \\
1.027 \pm 0.002\end{array}$ & $\begin{array}{l}1.000 \pm 0.007 \\
1.013 \pm 0.005 \\
1.022 \pm 0.005\end{array}$ \\
\hline $15 \mathrm{MeV}$ & $\begin{array}{c}\text { graphite/graphite } \\
\text { steel/graphite } \\
\text { brass/graphite }\end{array}$ & $\begin{array}{l}7.506 \pm 0.018 \times 10^{-13} \\
7.618 \pm 0.020 \times 10^{-13} \\
7.668 \pm 0.018 \times 10^{-13}\end{array}$ & $\begin{array}{l}108.18 \pm 0.54 \\
109.04 \pm 0.55 \\
109.42 \pm 0.55\end{array}$ & $\begin{array}{l}1.000 \pm 0.004 \\
1.015 \pm 0.004 \\
1.021 \pm 0.004\end{array}$ & $\begin{array}{l}1.000 \pm 0.005 \\
1.008 \pm 0.005 \\
1.011 \pm 0.005\end{array}$ \\
\hline
\end{tabular}

*air cavity dose per incident particle crossing the plane at SSD. 


\section{IV.D.4 Effect of electrode thickness}

The next question we addressed with Monte Carlo calculations is the effect of electrode thickness on the air cavity dose in our hybrid PEEC. We simulated the bone PEEC with two different polarizing/measuring electrode configurations: graphite/graphite and steel/graphite, and three different electrode thicknesses: $0.0025 \mathrm{~cm}, 0.005 \mathrm{~cm}$, and 0.01 $\mathrm{cm}$. The dose to the air cavity was calculated for the six possible setups for the $18 \mathrm{MV}$-ray beams. The results are presented in Table 8-7, and they show that the electrode thickness variation in the range from $0.0025 \mathrm{~cm}$ to $0.01 \mathrm{~cm}$, which was used in our experimental work, has essentially no effect on the air cavity dose.

Table 8-7. Dose to the chamber air cavity calculated with Monte Carlo techniques as a function of graphite electrode thickness for $18 \mathrm{MV}$ beam. All values are normalized to the $0.0025 \mathrm{~cm}$ values. The air cavity is at a depth of $6 \mathrm{~cm}$, field size $10 \times 10 \mathrm{~cm}^{2}$, SSD $100 \mathrm{~cm}$. The air cavity thickness is fixed at $2 \mathrm{~mm}$.

\begin{tabular}{|c|c|c|c|c|c|c|}
\hline Electrodes & \multicolumn{6}{|c|}{ Graphite electrode thickness $(\mathrm{cm})$} \\
\hline (polarizing/collecting) & \multicolumn{2}{|c|}{0.0025} & \multicolumn{2}{|c|}{0.005} & \multicolumn{2}{|c|}{0.01} \\
\hline & $\begin{array}{c}\text { Dose* } \\
\left(10^{-15} \mathrm{~Gy}\right)\end{array}$ & $\begin{array}{c}\text { Dose ratio } \\
\left(\frac{D_{\text {thickness }}}{D_{0.0025 \mathrm{~cm}}}\right)\end{array}$ & $\begin{array}{c}\text { Dose* } \\
\left(10^{-15} \mathrm{~Gy}\right)\end{array}$ & $\begin{array}{c}\text { Dose ratio } \\
\left(\frac{D_{\text {thickness }}}{D_{0.0025 \mathrm{~cm}}}\right)\end{array}$ & $\begin{array}{c}\text { Dose* } \\
\left(10^{-15} \mathrm{~Gy}\right)\end{array}$ & $\begin{array}{l}\text { Dose ratio } \\
\left(\frac{D_{\text {thickness }}}{D_{0.0025 \mathrm{~cm}}}\right)\end{array}$ \\
\hline graphite/graphite & $4.79 \pm 0.02$ & $1.000 \pm 0.0006$ & $4.77 \pm 0.02$ & $0.996 \pm 0.0006$ & $4.75 \pm 0.02$ & $0.992 \pm 0.0006$ \\
\hline steel/graphite & $4.98 \pm 0.02$ & $1.000 \pm 0.0006$ & $4.99 \pm 0.02$ & $1.001 \pm 0.0006$ & $4.92 \pm 0.02$ & $0.988 \pm 0.0006$ \\
\hline
\end{tabular}

*air cavity dose per incident particle crossing the plane at SSD.

\section{TOTAL CORRECTION FACTOR $\boldsymbol{C}_{\mathrm{CH}}$}

Having evaluated the possible effects on the air cavity dose of missing scatter, air cavity perturbations, and electrode configuration, we are now in a position to determine the total correction factor $C_{c h}$ to the dose in the air cavity measured with our hybrid PEEC. The correction factor $C_{c h}$ is a product of the individual correction factors, summarized in Table 8-8 for the five beam energies we used in our studies. $C_{c h}$ depends on beam type and energy and ranges from 1.066 for the cobalt-60 beam to 1.016 for the $15 \mathrm{MeV}$ electron 
beam. These factors were then used in our determination of dose with the hybrid PEEC in conjunction with Eq. (8-4).

Table 8-8. Dose correction factor $C_{c h}$ calculated with Monte Carlo techniques for bone PEEC at depth $d_{\max }$ for electron beams and at depth $6 \mathrm{~cm}$ for photon beams, field size $10 \times 10 \mathrm{~cm}^{2}$, SSD $80 \mathrm{~cm}$ for cobalt-60, SSD $100 \mathrm{~cm}$ for linacs beams. $C_{c h}$ also includes correction for humidity $k_{h}=0.997$.

\begin{tabular}{|c|c|c|c|c|c|c|}
\hline & IV.A. & IV.B. & IV.C. & IV.D. & & \\
\hline $\begin{array}{c}\text { Beam } \\
\text { quality }\end{array}$ & $\begin{array}{c}\text { Scatter } \\
\text { correction } \\
\text { factor }\end{array}$ & $\begin{array}{c}\text { Air cavity } \\
\text { perturbations }\end{array}$ & $\begin{array}{c}\text { Correction for } \\
\text { chamber wall }\end{array}$ & $\begin{array}{c}\text { Electrode } \\
\text { correction } \\
\text { factor }\end{array}$ & $\begin{array}{c}\text { Humidity } \\
\text { correction } \\
k_{h}\end{array}$ & $\begin{array}{c}\text { Total } \\
\text { correction factor } \\
C_{c h}\end{array}$ \\
\hline \hline Co-60 & $1.020 \pm 0.005$ & 1.000 & 1.000 & $1.048 \pm 0.005$ & 0.997 & $1.066 \pm 0.007$ \\
\hline $6 \mathrm{MV}$ & $1.015 \pm 0.007$ & 1.000 & 1.000 & $1.040 \pm 0.007$ & 0.997 & $1.052 \pm 0.009$ \\
\hline $18 \mathrm{MV}$ & $1.007 \pm 0.006$ & 1.000 & 1.000 & $1.024 \pm 0.004$ & 0.997 & $1.028 \pm 0.007$ \\
\hline $9 \mathrm{MeV}$ & $1.011 \pm 0.003$ & 1.000 & 1.000 & $1.012 \pm 0.002$ & 0.997 & $1.020 \pm 0.004$ \\
\hline $15 \mathrm{MeV}$ & $1.007 \pm 0.004$ & 1.000 & 1.000 & $1.012 \pm 0.004$ & 0.997 & $1.016 \pm 0.006$ \\
\hline
\end{tabular}

\section{DOSE MEASUREMENT IN BONE WITH THE HYBRID PEEC}

In Section IV. we showed that the hybrid PEEC cannot be used directly with Eq. (8-3) to determine the dose in bone-equivalent material. In subsequent sections we evaluated the necessary corrections to measured data to determine the dose-to-bone accurately. In Table 8-9 we summarize our measured and calculated data and show a good agreement between the measured data corrected with $C_{c h}$ and data calculated with Monte Carlo techniques. For photon beams the doses were measured at a depth of $6 \mathrm{~cm}$ and the readings were then corrected to $d_{\max }$ through the appropriate $P D D$ s. For the two electron beams the measurements were carried out at $d_{\max }$. Dose-to-bone was measured with the hybrid PEEC in conjunction with Eq. (8-4), dose-to-water with a Farmer cylindrical ionization chamber in conjunction with the AAPM TG-51 dosimetry protocol. All measured doses are normalized to $100 \mathrm{MU}$ for the linac beams and to 1 minute exposures for the cobalt- 60 beam. For the five radiation beams the appropriate restricted mass stopping power ratios are given in column (2) of Table 8-9, the measured ionization gradients in column (3) and the total correction factors $C_{c h}$ in column (4). The measured dose-to-bone 
calculated with Eq. (8-4) is given in column (5), the measured dose-to-water in column (6), and the ratio (dose-to-bone/dose-to-water) in column (7). This ratio is then compared to the same ratio calculated with Monte Carlo techniques and given in column (8).

The agreement between measured and calculated data is now on the order of $2 \%$ or better for all beams that we studied (column (9) of Table 8-9), leading us to conclude that Eq. (8-4) which incorporates the correction factors accounting for missing scatter and graphite electrode effects can be used in an experimental determination of absorbed dose-to-bone with our hybrid PEEC.

Table 8-9. Results of absolute calibration in bone-equivalent material carried out with bone PEEC using graphite electrodes. Dose is calculated with Eq. (8-4), $\rho=1.293 \times 10^{-3} \mathrm{~g} / \mathrm{cm}^{3}, A=8.019 \mathrm{~cm}^{2}$, $\bar{W}_{\text {air }}=33.97 \mathrm{~J} / \mathrm{C}$. $(\bar{L} / \rho)^{\text {bone }}$ is calculated with SPRRZnrc for each beam; $d Q / d z$ is measured at depth $d_{\max }$ for electron beams and at a depth of $6 \mathrm{~cm}$ for photon beams; ionization readings are corrected with the two voltage technique for ionic recombination and are also corrected for temperature, pressure and polarity effect. Dose (in cGy) is given at $d_{\max }$ (through appropriate percentage depth doses) for an exposure time of 1 minute for Co-60 (SSD $=80 \mathrm{~cm}$ ) and $100 \mathrm{cGy}$ in water for $10 \times 10 \mathrm{~cm}^{2}$ linac (SSD $=100 \mathrm{~cm}$ ) beams. The graphite electrodes are $0.005 \mathrm{~cm}$ thick and a $2 \mathrm{~cm}$ bone thickness on the back of the collecting electrode is used. $D_{b o n e}$ is corrected with $C_{c h}$ which includes corrections for lack of scatter and for graphite electrode perturbation as well as humidity correction.

\begin{tabular}{|c|c|c|c|c|c|c|c|c|}
\hline (1) & (2) & (3) & (4) & (5) & (6) & (7) & (8) & (9) \\
\hline $\begin{array}{l}\text { Beam } \\
\text { quality }\end{array}$ & $\begin{array}{c}(\bar{L} / \rho)^{\text {bone }} \\
\text { air } \\
\left.\text { (at depth } d_{\text {max }}\right)\end{array}$ & $\begin{array}{c}d Q / d z \\
\left(\text { at depth } d_{\max }\right) \\
\left(10^{-5} \mathrm{C} / \mathrm{m}\right)\end{array}$ & $C_{c h}$ & $\begin{array}{c}\text { Dose bone } \\
D_{b o n e} \\
\text { Eq. }(8.4) \\
{[\text { PEEC] }} \\
\text { (cGy) }\end{array}$ & $\begin{array}{c}\text { Dose Water } \\
D_{\text {water }}\left(d_{\max }\right) \\
\text { AAPM TG-51 } \\
\text { [Farmer] } \\
\text { (cGy) } \\
\pm 1 \% \\
\end{array}$ & $\begin{array}{l}\text { Measured } \\
\frac{D_{\text {bone }}}{D_{\text {water }}} \\
{\left[\frac{\operatorname{col}(5)}{\operatorname{col}(6)}\right]}\end{array}$ & $\begin{array}{l}\text { Monte } \\
\text { Carlo } \\
\frac{D_{\text {bone }}}{D_{\text {water }}}\end{array}$ & $\begin{array}{c}\text { Difference } \\
{\left[\frac{\operatorname{col}(7)-\operatorname{col}(8)}{\operatorname{col}(8)}\right]} \\
(\%)\end{array}$ \\
\hline Co-60 & 0.994 & 3.039 & 1.066 & $105.50 \pm$ & 109.2 & 0.966 & 0.949 & +1.8 \\
\hline $6 \mathrm{MV}$ & 0.984 & 2.944 & 1.052 & $99.83 \pm$ & 100.0 & 0.998 & 0.976 & +2 . \\
\hline $18 \mathrm{MV}$ & 0.959 & 3.195 & 1.028 & 103.21 & 100.0 & 1.032 & 1.042 & -0.96 \\
\hline $9 \mathrm{MeV}$ & 0.944 & 3.210 & 1.020 & $101.29 \pm 0.5 \%$ & 100.0 & 1.013 & 1.024 & -1.07 \\
\hline $5 \mathrm{MeV}$ & 0.933 & 3.206 & 1.016 & 99.57 & 100.0 & 0.996 & 0.993 & +0.30 \\
\hline
\end{tabular}




\section{Conclusions}

In this chapter we have developed the procedure for using our hybrid PEEC for accurate determination of absolute dose in non-tissue equivalent materials such as bone. The measurement of dose in biological materials other than tissue causes considerable difficulties with standard ionization chamber techniques similar to those used in water in conjunction with national or international dosimetry protocols. These difficulties result from unavailability of appropriate calibration and correction factors for materials other than water. Our initial approach for dose determination in bone-equivalent material with the hybrid PEEC followed the same approach as that used previously in Solid Water ${ }^{\mathrm{TM}}$ by Zankowski ${ }^{10,11,12}$. The results for dose-to-bone seemed reasonable, however, our attempts to confirm them with Monte Carlo calculations were not successful.

We then undertook an experimental and theoretical study of various possible effects which could have adversely affected our dose-to-bone measurements and concluded that, with an incorporation of scatter and electrode correction factors into the dose-to-bone measured data, we achieve a good agreement between experimental dose-to-bone data and data calculated with Monte Carlo techniques.

Our hybrid PEEC is thus capable of yielding accurate absolute dose to bone-equivalent materials without requiring a standards laboratory calibration factor. The dose determination procedure is based on the chamber signal after incorporating a correction factor determined through Monte Carlo techniques. The correction factor accounts for scatter perturbation in the hybrid PEEC and for effects of graphite electrodes on the dose in the chamber air cavity.

Our results show that the ideal hybrid PEEC would be made of bone completely, and incorporate a graphite polarizing electrode and an aluminum collecting electrode. In this design the bone-equivalent PEEC would not require any correction when determining absorbed dose to bone with the modified Spencer-Attix relationship. 


\section{References}

1 ICRU, "Determination of absorbed dose in a patient irradiated by beams of X or gamma rays in radiotherapy procedures," ICRU Report 24, International Commission on Radiation Units and Measurements, Washington D.C. (1976).

2 ICRU, "Measurement of absorbed dose in a phantom irradiated by a single beam of $\mathrm{X}$ or gamma rays," ICRU Report 23, International Commission on Radiation Units and Measurements, Bethesda, Maryland (1973).

3 G. Failla, "Measurement of tissue dose in terms of the same unit for all ionizing radiations," Radiology 29, 202-215 (1937).

4 D. J. Manson, D. Velkley, J. A. Purdy et al., "Measurements of surface dose using build-up curves obtained with an extrapolation chamber," Radiology 115 (2), 473-474. (1975).

5 R. Loevinger, "Extrapolation chamber for the measurement of beta sources," Sci. Instr. 24, 907-914 (1953).

6 J. Böhm and U. Schneider, "Review of extrapolation chamber measurements of beta rays and low energy x rays," Radiat. Prot. Dosim. 14, 193-198 (1986).

7 C. G. Soares, "Calibration of ophthalmic applicators at NIST: a revised approach," Med. Phys. 18 (4), 787-793. (1991).

8 S. Genna and J. S. Laughlin, "Absolute calibration of a cobalt-60 gamma-ray beam," Radiology 65, 394-405 (1955). 
9 S. C. Klevenhagen, "Determination of absorbed dose in high-energy electron and photon radiation by means of an uncalibrated ionization chamber," Phys. Med. Biol. 36 (2), 239-253 (1991).

10 C. E. Zankowski and E. B. Podgorsak, "Ionization gradient chamber in absolute photon and electron dosimetry," Radiology and Oncology (Slovenia) 30, 138-141 (1996).

11 C. E. Zankowski, "Calibration of photon and electron beams with an extrapolation chamber," Ph.D. Thesis, McGill University, 1997.

12 C. E. Zankowski and E. B. Podgorsak, "Calibration of photon and electron beams with an extrapolation chamber," Med. Phys. 24 (4), 497-503 (1997).

13 AAPM Task Group 21 of the Radiation Therapy Committee, "A protocol for the determination of absorbed dose from high-energy photon and electron beams," Med. Phys. 10, 741-771 (1983).

14 International Atomic Energy Agency, Absorbed dose determination in photon and electron beams: An international code of practice (IAEA Technical Reports Series No. 277, Vienna, 1987).

15 P. R. Almond, P. J. Biggs, B. M. Coursey et al., "AAPM's TG-51 protocol for clinical reference dosimetry of high-energy photon and electron beams," Med. Phys. 26 (9), 1847-1870 (1999).

16 L. V. Spencer and F. H. Attix, "A theory of cavity ionization," Radiat. Res. 3, 239-254 (1955). 
17 Chemical Rubber Company, CRC Handbook of Chemistry and Physics, 78th ed. (Chemical Rubber Pub. Co., Cleveland, 1997).

18 M. Boutillon and A. M. Perroche-Roux, "Re-evaluation of the W value for electrons in dry air," Phys. Med. Biol. 32, 213-219 (1987).

19 ICRU, "Stopping powers for electrons and positrons," ICRU Report 37, International Commission on Radiation Units and Measurements, Bethesda, Maryland (1984).

20 D. W. O. Rogers, "Fundamentals of dosimetry based on absorbed-dose standards," in Teletherapy Physics, Present and Future, edited by J.R. Palta and T.R. Mackie (Advanced Medical Publishing, 1996), pp. 319-356.

21 M. J. Berger and S. M. Seltzer, "Stopping powers and ranges of electrons and positrons," Report NBSIR 82-2550-A, National Bureau of Standards, Washington D. C. (1983).

22 Task group 25 American Association of Physicist in Medicine, "Clinical electron beam dosimetry," Med. Phys. 18, 73-109 (1991).

23 A. E. Nahum, "Extension of the Spencer-Attix Cavity Theory to the 3 media situation for Electron beams," in Dosimetry in Radiotherapy, edited by IAEA (IAEA, Vienna, 1988), Vol. 1, pp. 87-115.

24 S. C. Klevenhagen, "Implications of electron backscatter for electron dosimetry," Phys. Med. Biol. 36, 1013-1018 (1991).

25 S. C. Klevenhagen, "Electron backscattering. Implication to electron dosimetry," Radiol Med (Torino) 80 (4 Suppl 1), 160-162. (1990). 
26 M. A. Hunt, G. J. Kutcher, and A. Buffa, "Electron backscatter corrections for parallel-plate chambers," Med. Phys. 15 (1), 96-103. (1988).

27 C. M. Ma and D. W. Rogers, "Monte Carlo calculated wall correction factors for plane-parallel chambers in high-energy electron beams," Med. Phys. (abstract), 22, 672 (1995). 


\section{Chapter 9}

\section{Conclusions}

\section{Summary}

The main objective of the thesis work was to investigate phantom-embedded extrapolation chamber (PEEC) in absolute dosimetry of clinical megavoltage photon and electron beams. While Zankowski has already shown that an uncalibrated PEEC embedded in a Solid Water ${ }^{\mathrm{TM}}$ phantom can be used for absolute machine output measurements in Solid WaterTM, we have extended this work to PEEC embedded in bone-equivalent material and shown that with suitable corrections for phantom scatter and electrode effects we can accurately determine experimentally the dose-to-bone in a clinical megavoltage beam.

With the PEEC, the absolute dose in the phantom material is determined using the Spencer-Attix cavity theory in conjunction with ionization gradient measurements and an indirect determination of the chamber effective air-mass through a measurement of chamber capacitance which yields an accurate measure of the effective electrode area. We modified the original PEEC design by motorizing the chamber piston to automatize the data collection such that the relative electrode separations of the chamber can be varied by means of a stepping motor. This motor, as well as the associated IEEE-488 measurement devices are controlled by a personal computer. For measurements in bone we incorporated a bone entrance window, supporting the polarizing electrode, and a disc of bone material below the collecting electrode of sufficient thickness to produce backscatter radiation, equivalent to that obtained by a chamber fully embedded in bone-equivalent material. The chamber leakage current, as well as the uncertainty on the piston motion control were negligible. 
We used Monte Carlo techniques to model several megavoltage photon and electron beams. The results of these simulations were used to calculate dose and mean mass restricted stopping power ratios in the two phantom materials of interest, Solid Water ${ }^{\mathrm{TM}}$ and bone-equivalent material. The first direct dose-to-bone measurement results obtained with the hybrid PEEC seemed reasonable, however, when we tried to confirm them with Monte Carlo calculations, we obtained considerably different results. To improve our understanding of this discrepancy, we undertook a study of various parameters which might affect our dose-to-bone determination accuracy. These parameters were related to the chamber cavity as well as to the electrode configuration. Our study resulted in various correction factors which, together, correct the measured dose-to-bone and bring the results very close to Monte Carlo values. We found that the main factors affecting our dose measurements were scatter deficit and perturbations caused by the electrodes.

The correction factors for scatter deficit and electrode perturbations were calculated with Monte Carlo techniques. The latter effect was also verified through relative air cavity dose measurements with various electrode combinations. Scatter deficit, electrode perturbation and humidity were accounted for in the dose equation by adding a multiplicative factor $C_{c h}$ which depends on the beam energy and the materials that constitute the electrodes. By incorporating an appropriate $C_{c h}$ value into the dose relationship, dose-to-bone results became very close to Monte Carlo-determined values, therefore allowing us to use the hybrid PEEC for absolute dose measurements in heterogeneous materials.

The results obtained from the correction factor studies yielded other interesting conclusions. For example, missing scatter, due to geometry of the bone PEEC, is mainly caused by a lack of lateral scatter in the chamber. Graphite electrodes are appropriate for Solid Water ${ }^{\mathrm{TM}}$ dose measurements with the Solid Water ${ }^{\mathrm{TM}}$ PEEC, as it was assumed in Zankowski's work and shown by our Monte Carlo calculations, however, when they are used with the bone PEEC, correction factors to the measured signal must be applied to account for the differences in atomic numbers between bone and graphite. Monte Carlo calculations have shown that varying the thickness of thin electrodes does not have a large effect on the measured dose and that the material of the collecting electrode, in comparison to that of the polarizing electrode, produces a much larger effect. Finally, the optimum choice for dose-to-bone measurements with a PEEC is a phantom made completely of 
bone-equivalent material used in conjunction with a graphite polarizing electrode and an aluminum measuring electrode.

We also investigated the validity of the saturation curve model developed by Zankowski for pulsed beams with several ionization chambers (NE2517, Capintec PR-06, Holt, and PEEC). For ionization chambers exposed to pulsed megavoltage photon and electron beams, the linearity of the $1 / I$ and $1 / V$ plot breaks down in the polarizing voltage range where chambers are normally operated. The breakdown is caused by charge multiplication in the chamber sensitive volume and by leakage currents produced by radiation in the chamber stem, connector, and cable. The magnitude of this effect varies from one chamber to another and is also strongly dependent on field size. The charge multiplication component depends exponentially on $V$, while the radiation-induced leakage component depends linearly on $V$. Both components are approximated with an exponential term $e^{\gamma V}$ in Boag's saturation curve equation.

Depending on the particular ionization chamber model, the breakdown in the linearity between $1 / I$ and $1 / V$ may cause an overestimation of the radiation beam output by $\sim 0.7 \%$ for a $10 \times 10 \mathrm{~cm}^{2}$ field and by a few percent for large radiation fields. Overestimations occur when the standard "two-voltage" technique is used for determination of the saturation current. Therefore the "two-voltage" technique should be used with caution, and its reliability for a particular ionization chamber should be well established before the particular chamber is used in radiation dosimetry.

\section{Future work}

Work is underway to simplify the dose measurement process with PEEC. Characterization of ionic recombination in the chamber cavity as a function of electrode separation should make possible a direct dose measurement. Integration of collected charge, while the chamber electrodes spacing is varied, could directly provide the ionization gradient $(d Q / d z)$ required by the dose equation. 
To further understand and expand the saturation curve model for the PEEC, the amount of initial recombination could be estimated by keeping the chamber electric field constant as the cavity gap changes.

Other biological-equivalent materials could be used to create different hybrid PEEC configurations, such as lung-equivalent material. A study of the cavity of a lung-PEEC with Monte Carlo techniques would be required, as it was for bone-PEEC and large dose correction factors would be needed because of the large difference in composition between a suitable electrode material and the lung material which has a very low density $\left(\rho \approx 0.3 \mathrm{~g} / \mathrm{cm}^{3}\right)$.

Further theoretical and experimental investigations with various electrode configurations should lead to more accurate correction factors and to a better understanding of the effect on and its relations to the parameters on which it depends. It would be interesting to look at the electrode effect as a function of the air cavity size and also as a function of the electrode diameter. The precision of the dose correction factors could be improved by running more histories in the Monte Carlo calculations.

Finally, more clinical beams could be modeled with the BEAM code in order to expand our measurements to other photon and electron energies. This would provide a larger range of energies on which the various dose correction factors and mean mass restricted stopping power ratios could be calculated. 


\section{Appendix 1}

\section{IEEE-488 instrument control}

This Appendix contains two example programs to perform the following tasks with the PEEC system:

1. PEEC automatic electrode area determination

2. Saturation curve measurement with reference chamber (also used for leakage current measurement)

\section{PEEC automatic electrode area determination}

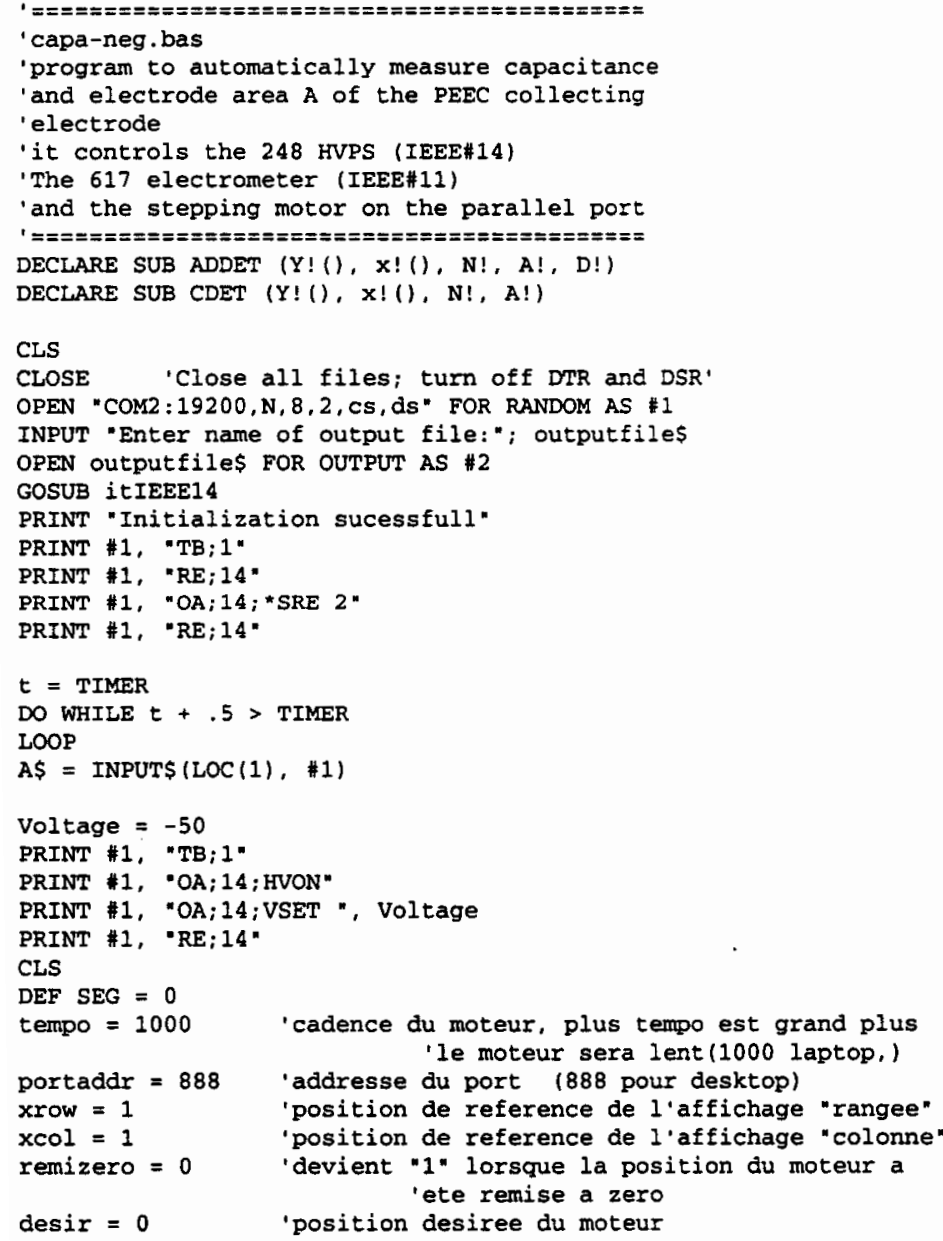




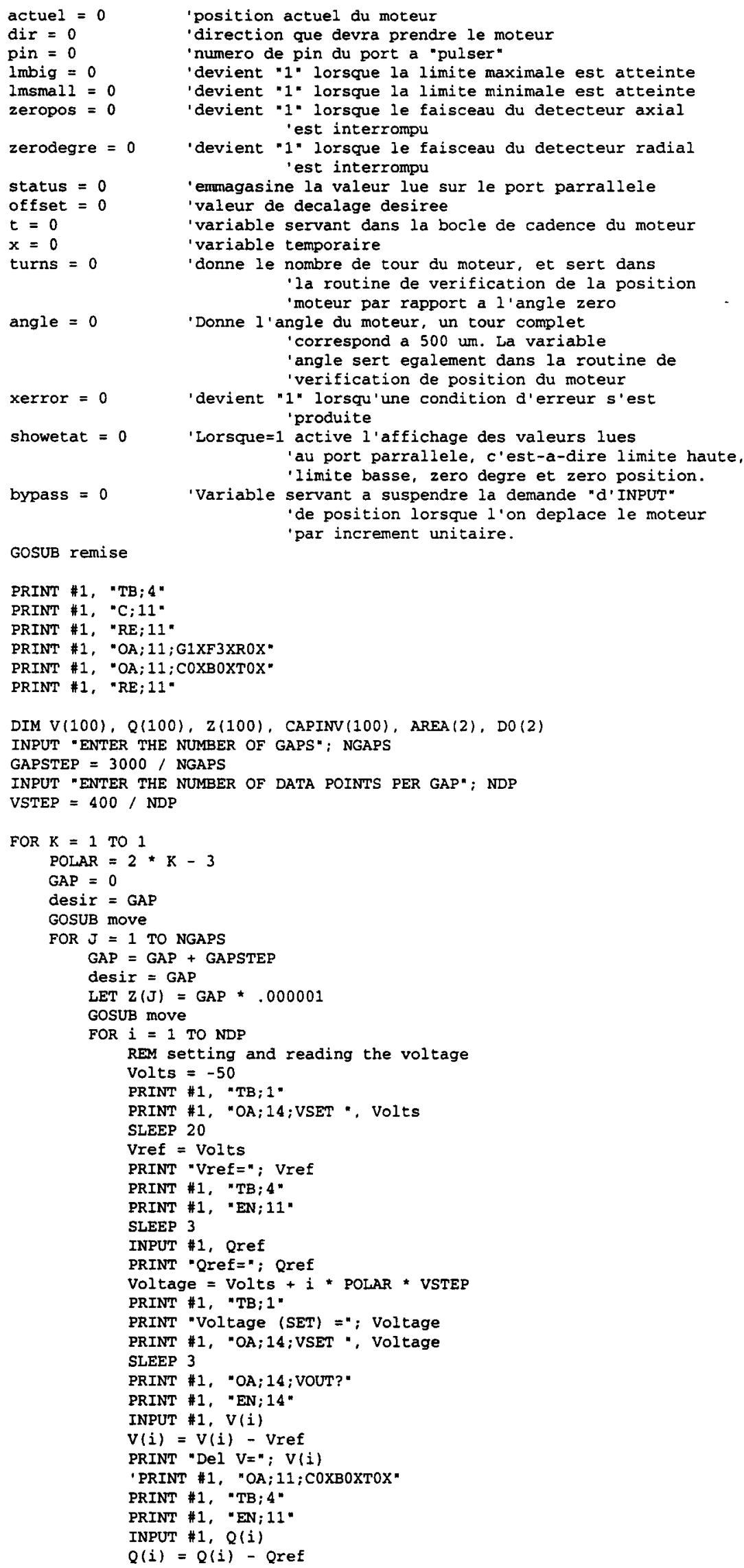




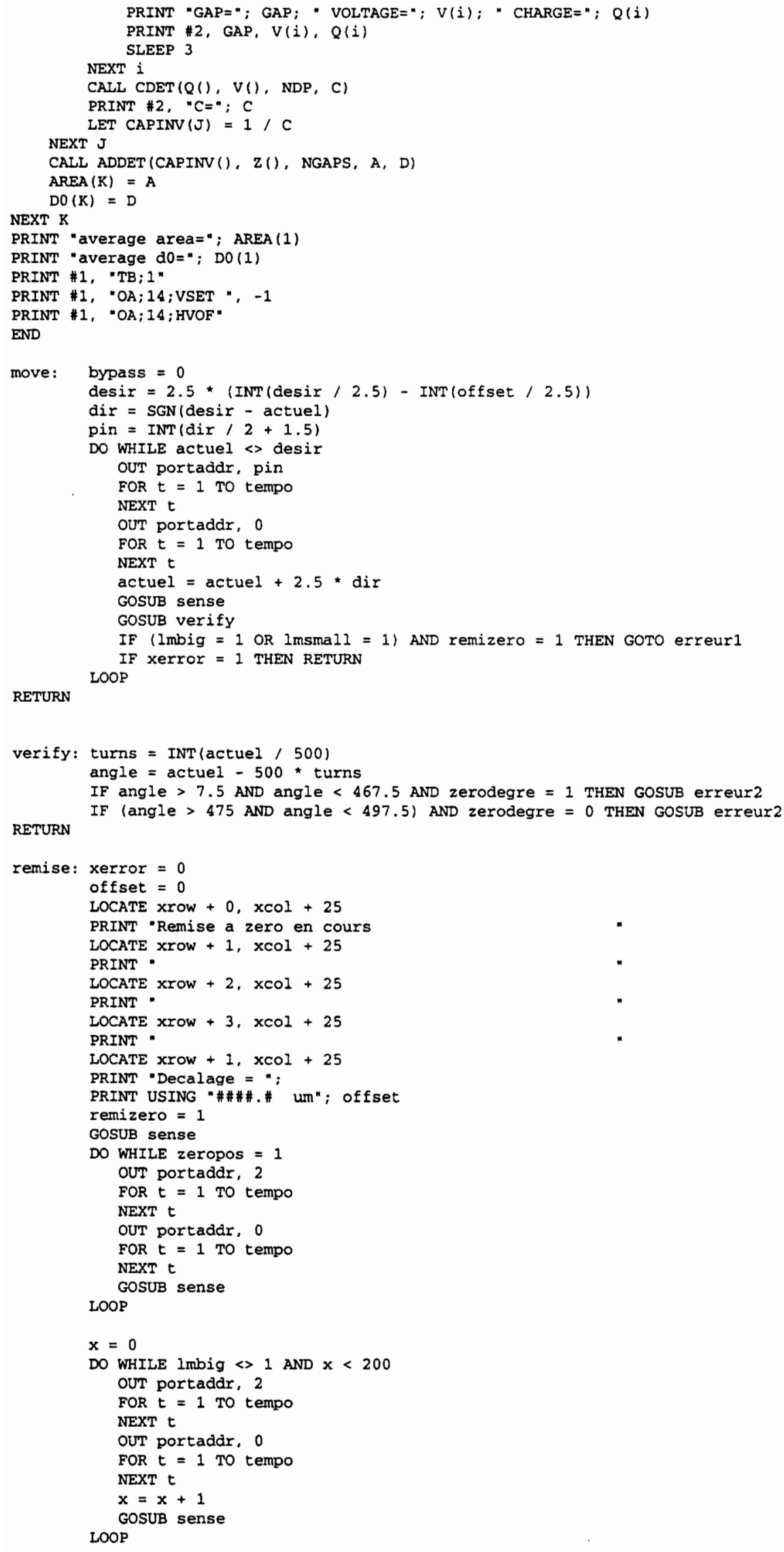




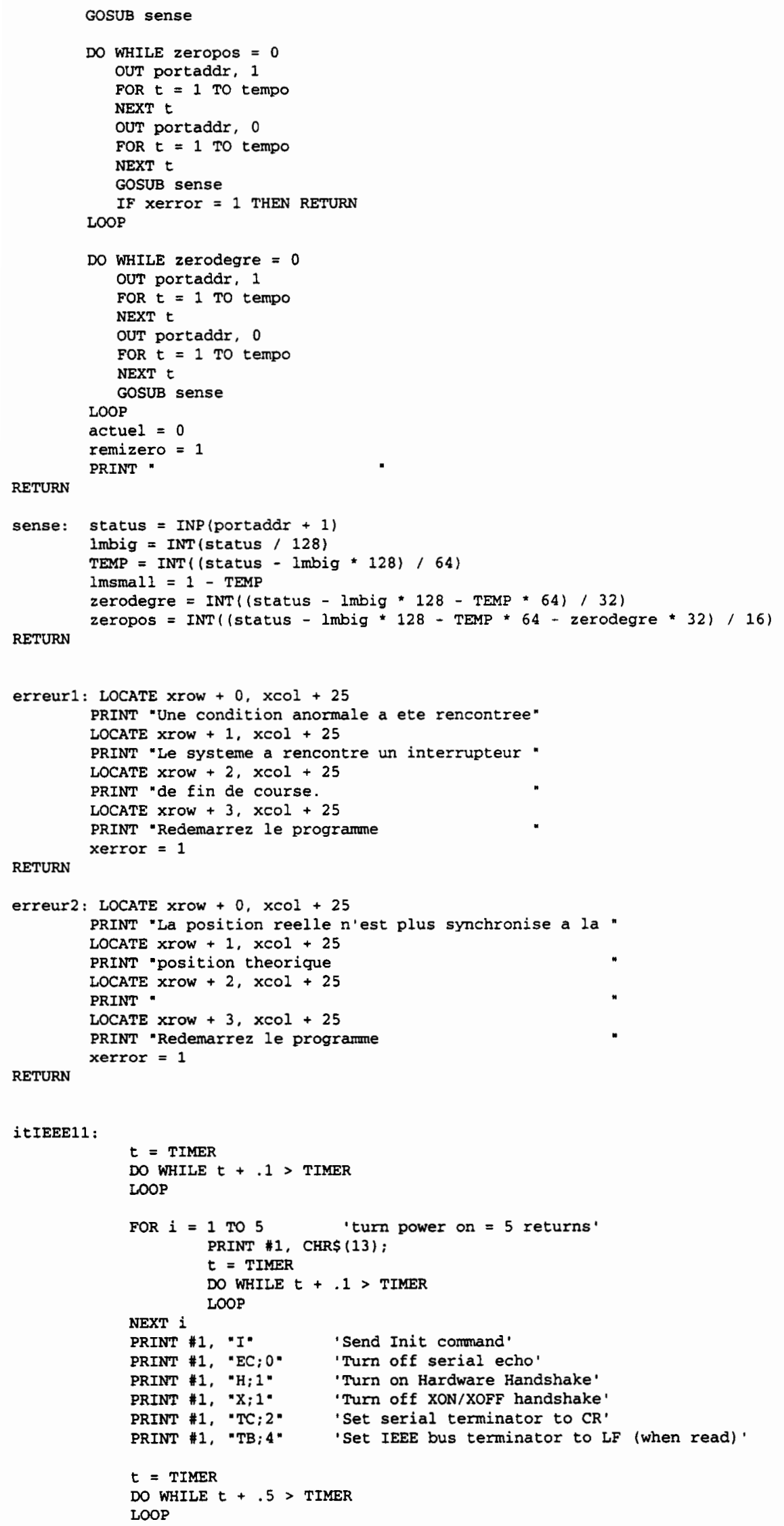




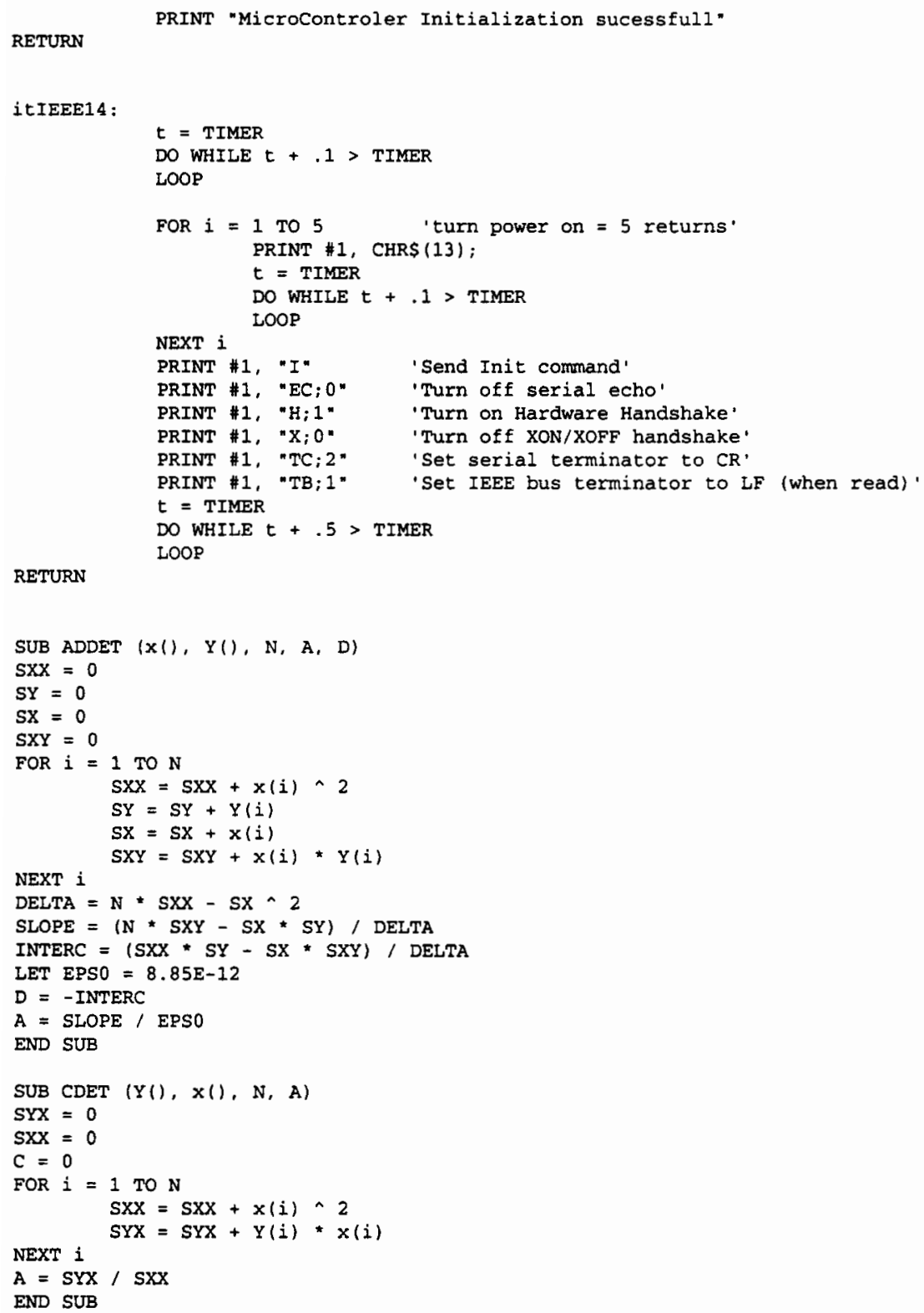




\section{Saturation curve measurement}

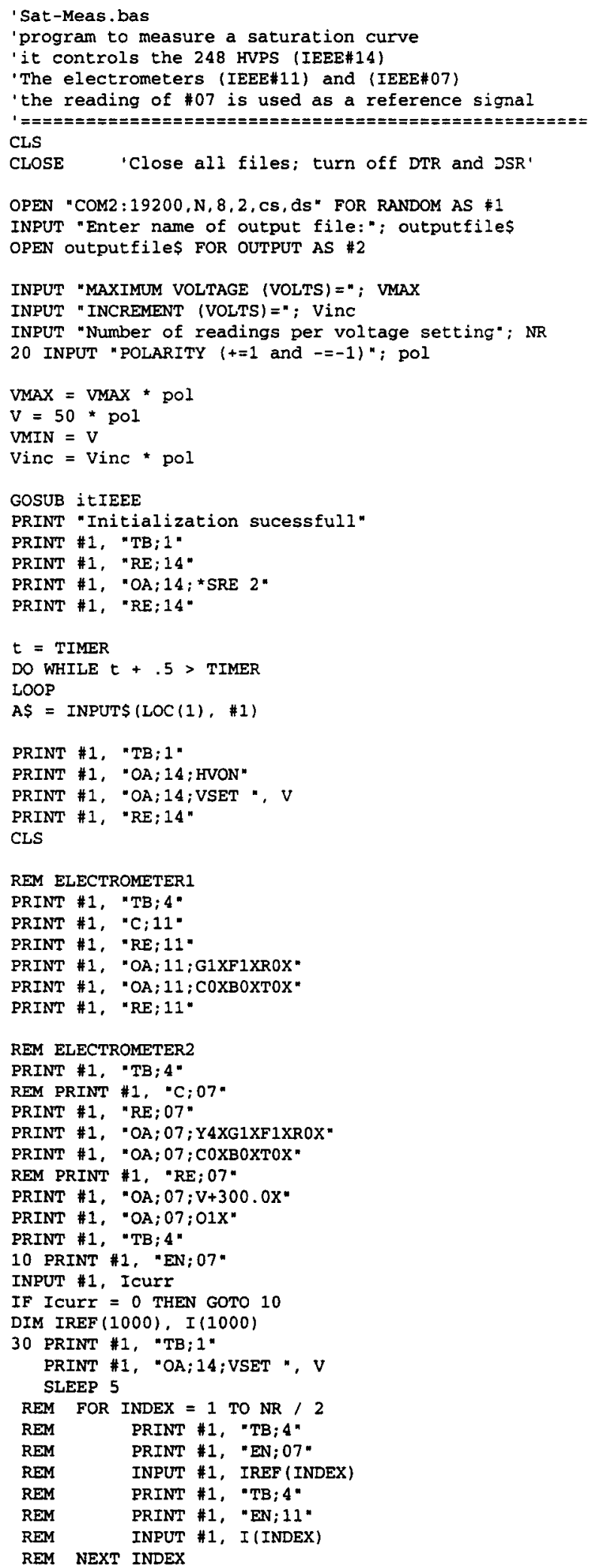




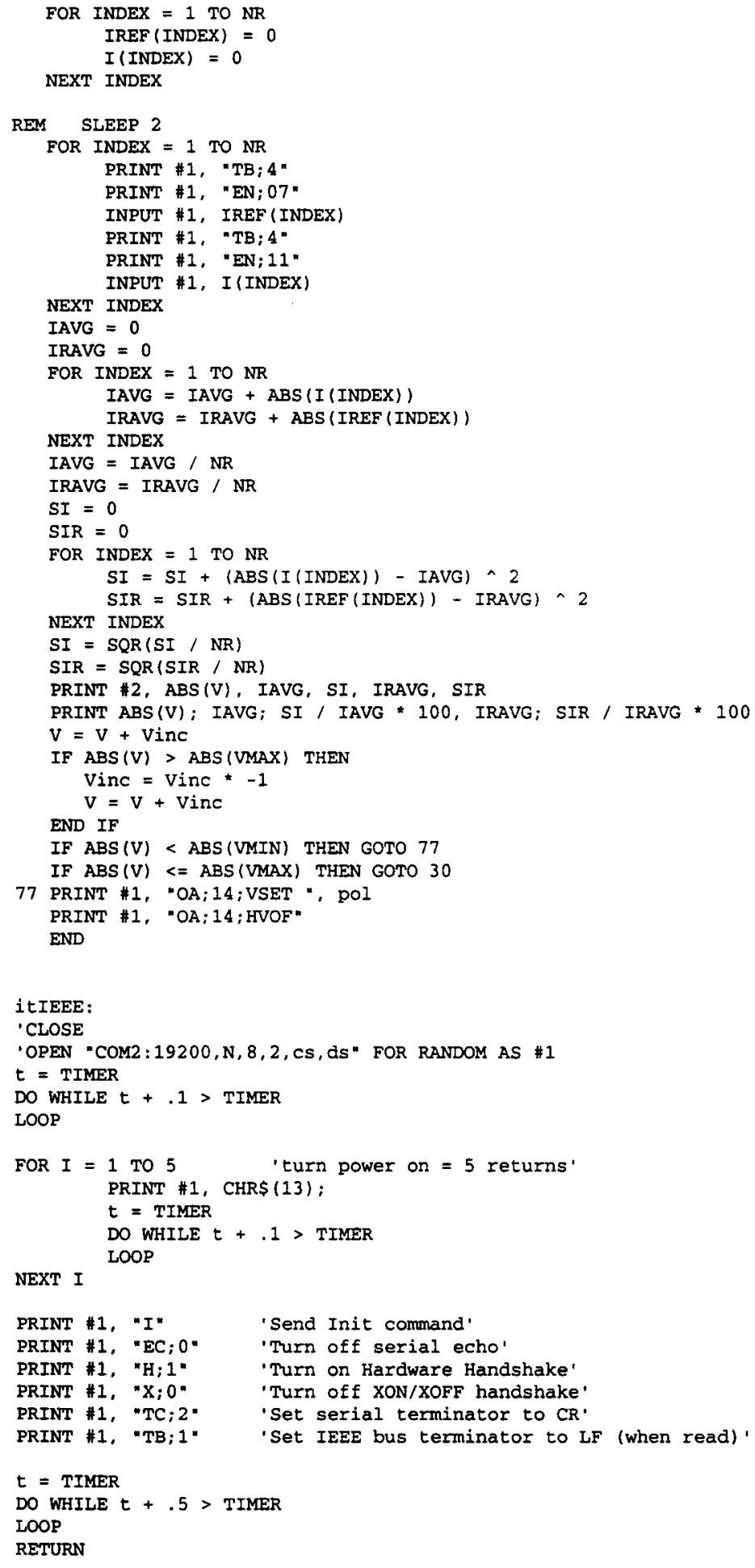




\title{
Appendix 2
}

\section{Stepping motor user guide}

\author{
(in French)
}

\begin{abstract}
Moteur pas à pas
II est possible à l'aide d'un moteur relié à la vis d'ajustement de faire varier l'espacement à distance. L'opérateur n'est plus obligé d'aller dans la salle où se trouve la source afin d'effectuer un ajustement manuel. Cependant il devient nécessaire de s'assurer de l'espacement exact entre les électrodes et ce sans voir les indications du micromètre. II est possible de mettre en place un appareil de mesure à distance de l'espacement. Cependant les variations sont très petites, et elles nécessiteraient des capteurs d'une haute précision

Connaissant le pas de la vis à $0.5 \mathrm{~mm}$, il est possible de connaître l'espacement exact entre les électrodes dans la mesure où nous sommes capable de commander précisément le nombre de tours ou de fraction de tours effectués par le moteur. Un moteur pas à pas devient donc le candidat idéal pour une telle application. II suffit de gérer adéquatement le nombre et la direction des pas effectués par le moteur afin d'en connaître en tout temps sa position exacte

Les appareils de contrôle de la chambre à ionisation PEEC sont déjà conçu pour être relié à un "PC". On peut via l'interface série et un contrôleur IEEE-488 commander toutes les fonctions des électromètres et de la source de voltage. Ceci permet aussi le renvoi au "PC" de l'ensemble des résultats des mesures effectuées pour archivage et usage ultérieur. Le port parallèle sera utilisé afin de commander le moteur pas à pas. Ceci présente l'avantage que toutes les fonctions de commandes et contrôles sont effectuées à partir d'un seul appareil.

Le port parallèle n'est pas en mesure de piloter directement le moteur pas à pas. Une interface est nécessaire afin de fournir les niveaux de tensions ainsi que les courants appropriés. Il est cependant facile de concevoir un programme pouvant gérer les "impulsions" à envoyer à cette interface et ainsi commander la position du moteur
\end{abstract}

\section{Description mécanique}

Un bloc servant à supporter le moteur est libre de glisser de haut en bas suivant les deux rails guides. La partie supérieure de l'axe du moteur est fixée à la vis micro-métrique. Sur la partie inférieure de l'axe du moteur est fixé l'obturateur qui à chaque rotation complète du moteur vient s'engager entre la diode électroluminescente et le photo-transistor. Ce photo-détecteur est l'élément du montage qui indique la position du moteur lors d'une rotation de $\mathbf{3 6 0}$ degrés. Un autre photo-détecteur situé au dessus du bloc de support du moteur est fixé à l'un des rails guides. Un obturateur est fixé sur la partie supérieure du bloc de support du moteur et vient s engager entre la diode électroluminescente et le phototransistor lorsque le moteur entraîne son bloc support vers le haut. Il y a deux interrupteurs de fin de course fixés à l'un des rails guides. L'un est situé au dessus du bloc de support et l'autre en dessous. Donc, si le moteur entraîne son bloc support trop haut ou trop bas dans sa course, celui-ci viendra activer un des deux interrupteurs. 


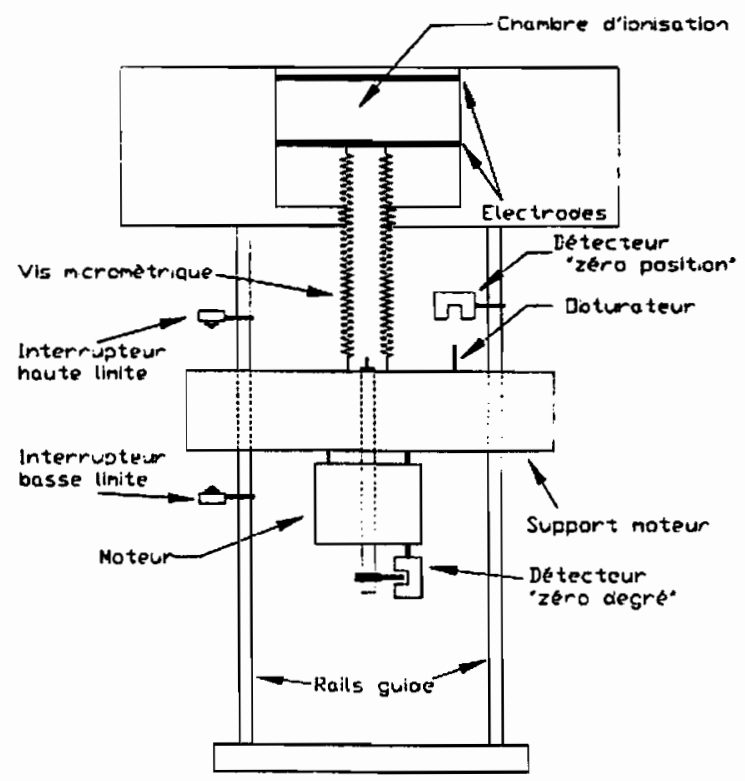

Figure 1.

\section{Principe de base d'un moteur pas à pas}

La représentation a plus simple d'un moteur pas à pas est tel que la figure 2. Celui-ci consisterait en quatre bobines reliées électriquement en paires de la façon indiquée. En appliquant une polarité particulière sur chacune des deux paires de bobines on obtiendrait un champ magnétique d'une polarité donnée pour chaque paire. Le champ magnétique résultant serait donc tel qu'indiqué. Si on inverse la polarité électrique d'une paire de bobines, on inverse donc la polarité de son champ magnétique (voir $2 b$ ). Le champ magnétique résultant de l'addition des champs des deux paires de bobines subit donc une rotation de 90 degrés par rapport au précédant (voir différence entre $2 a$ et $2 b$ ). Maintenant si nous inversons la polarité de l'autre paires de bobines nous obtenons encore une rotation de 90 degrés (voir $2 e$ ). Si nous revenons ré-inverser la polarité de la première paire nous obtenons encore une nouvelle rotation de 90 degrés (voir $2 \mathrm{~d}$ ). Si nous continuons ce manège en alternant successivement la polarité sur l'une et 'autre des paires nous pourront faire tourner notre moteur. Bien entendu le fait de faire tourner notre champ magnétique ne constitue pas un moteur, mais si nous plaçons au centre de ce montage un aimant permanent fixé à un axe, cet axe fera un tour complet sur lui-même à chaque quatres alternances de notre polarité électrique. Pour faire tourner le moteur dans le sens contraire il suffit que de commencer la séquence par l'autre paire de bobines. Ce moteur aurait quatre pas ou position par rotation de $360^{\circ}$. 


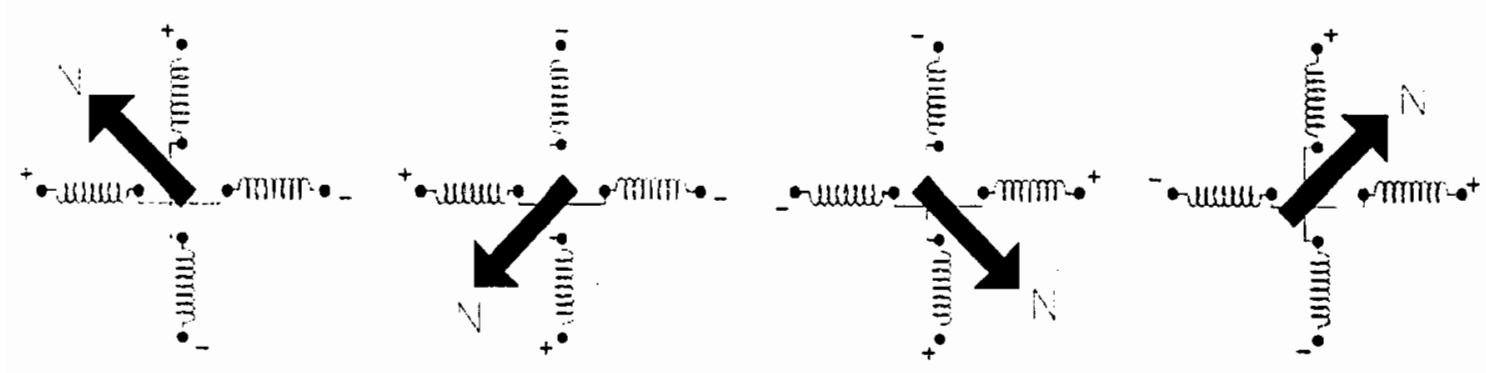

Figure 2.

Maintenant si l'on disposait d'un plus grand nombre de bobines à la façon de la Figure 3 , il serait possible d'obtenir un moteur ayant beaucoup plus de pas pour chaque rotation de $\mathbf{3 6 0}$ degrés. En observant la première situation de la Figure 3 , on peut voir que si l'on inverse a polarité du groupe de bobines $\mathrm{A}$, le rotor se déplacera dans une direction anti-horaire, situation 2 . Si au contraire on inversait la polarité du groupe de bobines $B$, on se retrouverait dans la situation 3 , et le moteur aurait donc subit un déplacement dans le sens horaire. Le moteur utilisé dans le présent montage possède 200 pas, donc chaque pas représente une rotation de 1.8 degré. Ce moteur fut récupéré d'un système de positionnement de têtes d'une unité de disque dur. II possédait déjà un détecteur photo-électrique permettant de déterminer lorsqu'il faisait un tour complet.
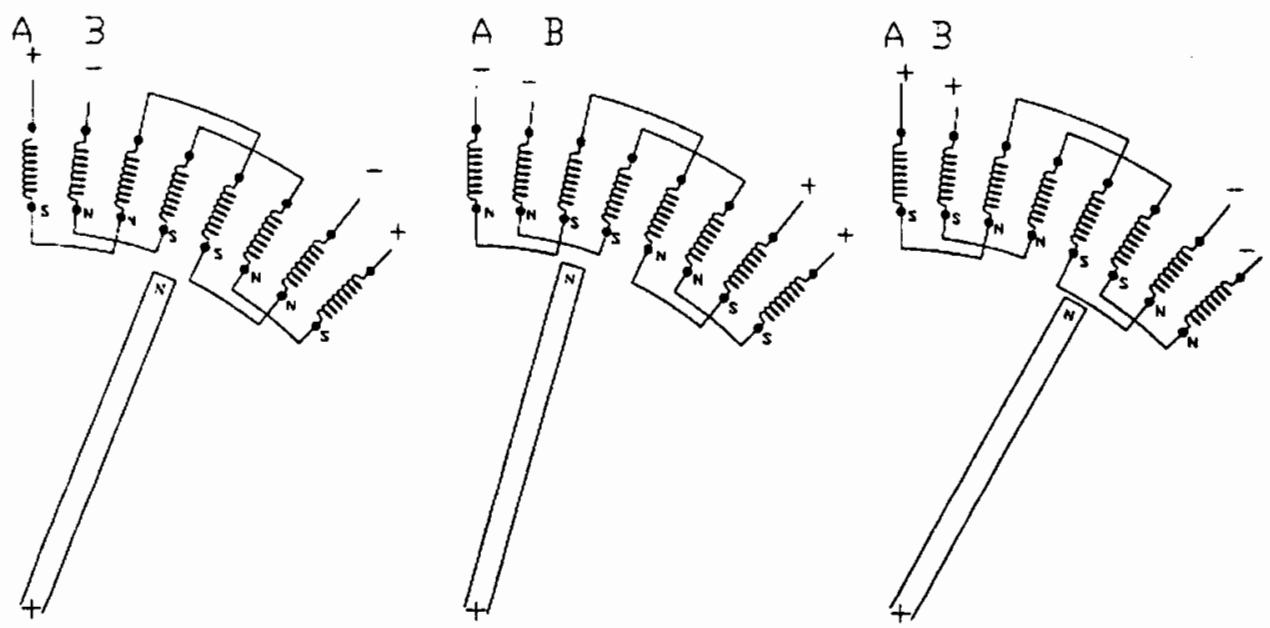

Figure 3.

\section{Boite d'interface}

La boîte d'interface recevra les signaux du port parallèle du PC" et les traitera de façon à déplacer le moteur tels que désiré. De plus, l'interface recevra des signaux des différents capteurs du montage moteur de façon à indiquer au programme de commande du moteur les actions à prendre.

La boîte d'interface se compose de : l'étage d'entrée, consistant en deux portes logiques "ET", une pour chaque direction de rotation. Un séquenceur de polarisation" qui mémorise la polarité que 
doit avoir chacune des bobines du moteur, et qui détermine en fonction de la rotation désirée, comment doit changer cette polarisation. Deux amplificateurs opérationnels servant de traducteur de niveaux logiques, c'est-à-dire $0-5$ volts vers -5 et +5 volts. Deux paires de transistors servant à fournir aux bobines le courant nécessaire à celle-ci. On y retrouve également quelques portes logiques tampons, afin d'adapter les impédances de différentes composantes.

Le connecteur de la boîte d'interface fut choisi de façon à permettre l'usage d'un câble d'imprimante conventionnel entre celle-ci et la sortie parallèle du "PC". Au niveau du connecteur de la boîte d'interface, les broches sont utilisées de la façon suivante. Seule 7 des 36 broches disponibles sont nécessaires. (Voir tableau 1)

\begin{tabular}{|c|c|l|}
\hline $\begin{array}{l}\text { Broche de la connexion } \\
\text { "Imprimante" }\end{array}$ & $\begin{array}{l}\text { Broche du connecteur } \\
\text { J1-P1 du circuit imprimé } \\
\text { de la boîte d'interface }\end{array}$ & Fonction \\
\hline 2 & 1 & Impulsion "Rapprocher les électrodes" \\
\hline 7 & 2 & Limite haute \\
\hline 3 & 3 & Impulsion "Écarter les électrodes" \\
\hline 8 & 4 & Limite basse \\
\hline 9 & 5 & Détecteur "zéro position" \\
\hline 10 & 6 & Détecteur "zéro degré" \\
\hline 36 & 7 & Masse \\
\hline
\end{tabular}

Tableau 1.

La boîte d'interface est reliée au montage de la chambre d'ionisation à l'aide d'un câble à 9 conducteurs munis de connecteurs type DB9. L'utilisation des broches est telle qu'illustré au tableau 2. La numérotation est la même que pour les connecteurs J2-P2 du circuit imprimé.

\begin{tabular}{|c|l|}
\hline Broches & Fonctions \\
\hline & Alimentation de la bobine ayant les fils jaune et blanc \\
\hline 2 & Alimentation de la bobine ayant les fils rose et bleu \\
\hline 3 & Signal de retour limite haute (écart minimum entre les électrodes) \\
\hline 4 & Commun des deux bobines ( +6 volts) \\
\hline 5 & Commun des signaux logiques (0 volt) \\
\hline 6 & Signal de retour limite basse (écart maximum entre les électrodes) \\
\hline 7 & Signal de retour "zéro degré" \\
\hline 8 & Signal de retour "zéro position" \\
\hline 9 & Alimentation en courant des diodes électroluminescentes des photo détecteurs \\
\hline
\end{tabular}

Tableau 2. 


\section{Etalage d'entrée}

Consiste en deux portes logiques "ET" à deux ports, une pour chaque direction de rotation. Un des ports pour chacune des deux portes est reliés au port parallèle du "PC". Pour chaque "impulsion" envoyée par le "PC", l'impulsion sera transmise au séquenceur de polarisation, en autant que l'interrupteur de fin de course n'a pas été activé. Si l'interrupteur de fin de course est enfoncé, la porte aura un "zéro" sur l'une de ses entrées, la sortie sera donc "zéro" en tout temps, et l'impulsion ne sera pas transmise.

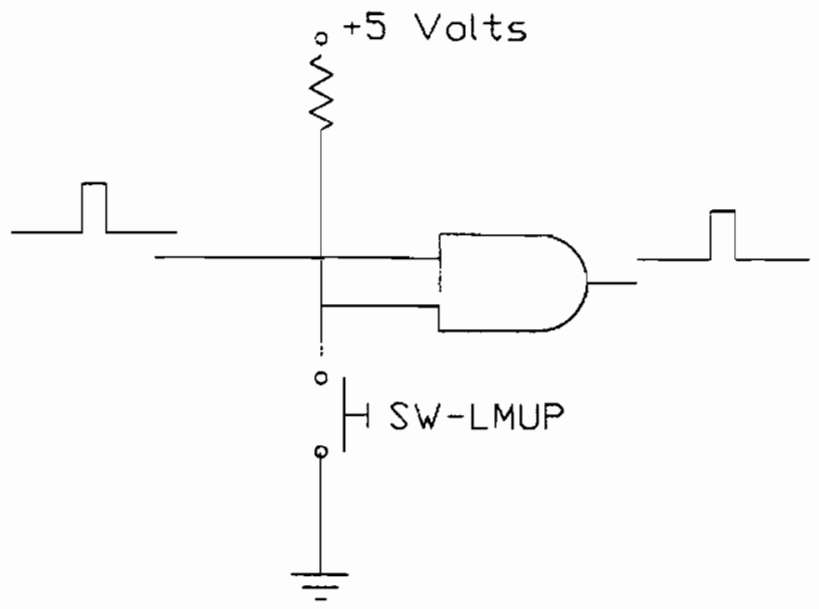

Figure 4.

Séquenceur de polarisation

Consiste en deux registres à deux bits de type " $D$ ", référence U2', et un sélecteur à quatre portes "MC14519" dont deux portes sont utilisées. Les deux registres de types " $D$ ' servent à maintenir la polarité des deux bobines. La logique est telle que pour une impulsion sur une ligne ou l'autre, un seul registre change de valeur. La figure 2 représente la logique du séquenceur. Si nous débutons avec une condition ou les deux registres contiennent la valeur logique zéro, et que nous envoyons une impulsion sur la porte A, le registre Q1 prendra la valeur inverse de Q2, donc 1, et le Q2 la valeur de Q1. (Voir tableau 1) 


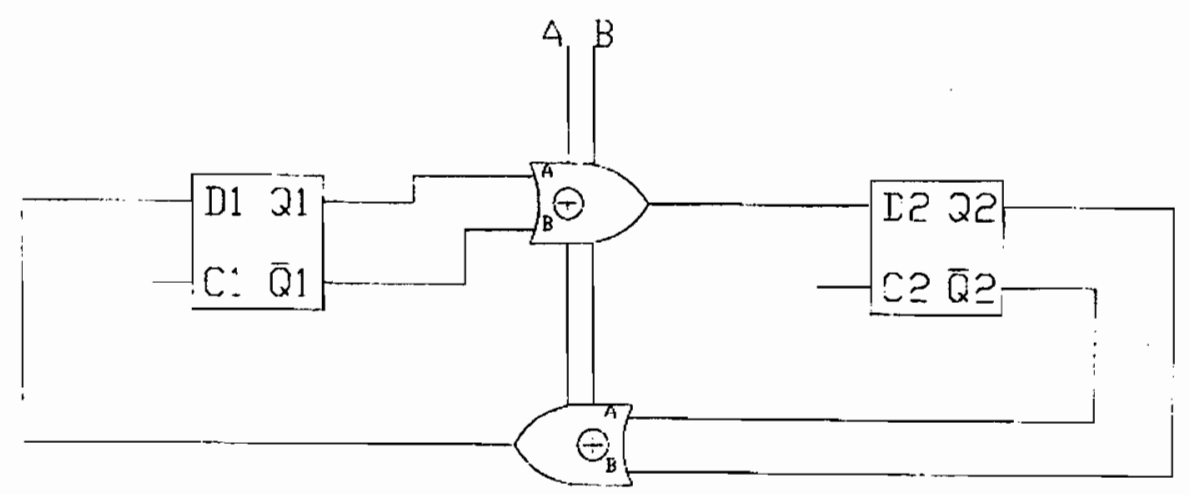

Figure 5.

\begin{tabular}{|l|l|}
\hline Q1 & Q2 \\
\hline 0 & 0 \\
\hline 1 & 0 \\
\hline 1 & 1 \\
\hline 0 & 1 \\
\hline 0 & 0 \\
\hline
\end{tabular}

Tableau 3.

\begin{tabular}{|l|l|}
\hline Q1 & Q2 \\
\hline 0 & 0 \\
\hline 0 & 1 \\
\hline 1 & 1 \\
\hline 1 & 0 \\
\hline 0 & 0 \\
\hline
\end{tabular}

Tableau 4.

Une deuxième impulsion sur la porte $A$ nous donnera $Q 1$ et $Q 2$ égale à 1 (voir ligne 3 du tableau 3 ). Ainsi de suite nous reviendrons à la condition $Q^{\circ}=0$ et $Q_{2}=0$. Si au contraire nous appliquons les impulsions à la porte $B$, nous obtiendrons la séquence du tableau 4 , qui en fait est la séquence du tableau 3 en sens inverse. Ce qui revient à la situation suivante : 


\begin{tabular}{|l|l|}
\hline Q1 & $Q 2$ \\
\hline 0 & 0 \\
\hline 1 & 0 \\
\hline 1 & 1 \\
\hline 0 & 1 \\
\hline
\end{tabular}

Tableau 5.

Ceci nous donne donc les deux séquences nécessaires pour faire tourner notre moteur pas à pas soit dans un sens ou dans l'autre. Donc quelque soit la polarité des bobines à un moment donné, le circuit séquenceur assurera que la polarité sera inversée sur la bobine appropriée afin de déplacer le moteur dans la direction désirée.

\section{Amplificateurs opérationnels (traducteur de niveaux logiques)}

Les tensions disponibles à la sortie des registres " $D$ " ont des valeurs de 0 ou 5 volts représentants la polarisation que doivent avoir les bobines du moteur pas à pas. Cependant le moteur lui doit opérer avec des valeurs de +6 ou -6 Volts. La source de tension a donc été conçue pour fournir des tensions de +6 et +12 volts pour les bobines du moteur, une tension de +5 volts pour les circuits logiques. Les deux bobines ont donc une borne commune reliée à +6 volts, alors que leur autre borne est reliée à une paire de transistors dont la "sortie" peut être commutée à 0 ou 12 volts. Le passage de 0 à 12 volts permet donc effectivement d'inverser la polarité sur chacune des bobines. En comparant la tension de sortie des deux registres " $D$ " à une source de 2.5 volts, les amplis peuvent donc commuter la tension aux bases des paires de transistors à une valeur près de 0 volts ou une près de 12 volts. La source de 2.5 volts est obtenue à l'aide d'un diviseur de voltage composé des résistances "R3" et "R4".

\section{$\underline{\text { Transistors de sorties }}$}

Les quatre transistors sont montés en paire, en configuration "Push-Pull", et permettent de fournir le courant nécessaire aux bobines du moteur. Les amplificateurs opérationnels n'ayant nullement la capacité de fournir un tel courant, approximativement $1 / 2$ ampères.

\section{Résistances, tampons et autres}

En plus des composantes précédemment décrites, nous retrouvons: un transformateur avec un pont de diodes, trois régulateurs de tensions et plusieurs condensateurs servant à l'alimentation des circuits logiques et du moteur.

La résistance $\mathrm{R} 5$ sert de source de courant aux deux diodes électroluminescentes des détecteurs photoélectriques servant dans la détection de la position de référence de la chambre.

Les résistances R6 et R7 servent de "pull-up" aux transistors de ces mêmes détecteurs.

Les résistances Ri et R2 servent de "pull-up" dans le circuit d'interrupteur de fin de course haute limite et basse limite.

Les diodes D2 et D3 servent de porte logique "OU". 
Le circuit composé de R8, $\mathrm{C} 1$ et d'une des portes tampons de U4, sert à créer un délais dans l'application de l'impulsion aux broches CLK1 et CLK2 de U2.Ce circuit à pour effet de s'assurer de d'un état stable des signaux de U1 avant d'envoyer le "coup d'horloge" à U2.

\section{Programmation et principe de fonctionnement}

\section{Port parallèle}

Le port parallèle d'un "PC" permet jusqu'à 13 lignes de sorties et 5 lignes d'entrées de valeurs binaires. Lorsque utilisé avec une imprimante, ces lignes servent entre autres à la transmission d'un byte, la valeur ascii du caractère à être imprimé, une ligne de validation du caractère transmis, une ligne d'initialisation de l'imprimante, etc. Le port reçoit également de l'imprimante des signaux indiquant son état ; l'imprimante n'a plus de papier, l'imprimante est prête à recevoir un autre caractère, ou encore un message d'erreur. Dans notre cas, nous n'utiliserons que deux lignes de sorties et quatre lignes d'entrées. Le port parallèle s'avère donc plus que suffisant.

Une des lignes de sorties sert à envoyer une impulsion qui fera se déplacer le moteur d'un pas dans une direction, alors que l'autre le fera se déplacer dans l'autre direction. Deux des entrées serviront aux interrupteurs de fin de course limite haute et limite basse, alors que la troisième servira à indiquer la position de départ ("position zéro"), et la quatrième le point de référence de rotation du moteur ("zéro degré").

\section{Lecture et écriture au port parallèle}

Le byte "état de l'imprimante" peut être obtenu à l'aide de l'instruction appropriée dans un langage quelconque de programmation à l'adresse décimale 889 . Les cinq bits les plus significatifs indique à l'ordinateur différentes conditions de l'imprimante. Les trois autres sont toujours zéro. Une simple formule mathématique permet d'extraire la valeur de chacun des bits et de l'assigner à une variable spécifique. L'assignation est la suivante :

\begin{tabular}{|l|l|l|}
\hline Poids du bit & Nom de la variable & Fonction \\
\hline 7 & LMDW & Interrupteur de fin de course basse \\
\hline 6 & LMUP & Interrupteur de fin de course haute \\
\hline 5 & ZEROPOS & Détecteur de position de référence "grossière" \\
\hline 4 & ZERODEG & Détecteur tour complet du moteur \\
\hline
\end{tabular}

Tableau 6

\section{Programmation "BASIC"}

Le langage choisi pour le contrôle de l'interface est le QuickBasic pour sa simplicité. Lorsque le programme est lancé pour la première fois, une routine d'initialisation permet de positionner le système à un point de référence. Le programme commande au moteur de tourner dans la direction permettant d'agrandir l'espacement entre les électrodes. Ceci assure donc le dégagement du détecteur de "position zéro" (voir diagramme schématique du montage). Le programme envoie au moteur des impulsions le faisant tourner en sens contraire, refermant ainsi l'espace de la cavité. II est important de savoir qu'après chaque impulsion, donc à chaque fois que le moteur se déplace d'un pas, le programme vient lire le port parallèle afin de déterminer l'état des différents détect- 
eurs. Aussitôt qu'est détectée la condition "zéro position", le programme cesse de réduire l'écart entre les électrodes. Ceci nous donne une position de référence grossière. En effet considérant que le pas de la vis est de $0.5 \mathrm{~mm}$ et que le moteur fait 200 pas par rotation complète, ceci implique que l'écart des électrodes varie de $0.5 \mathrm{~mm} / 200=2.5 \mu \mathrm{m}$ pour chaque pas de moteur. Le moindre déplacement de l'obturateur du coupleur photoélectrique fausserait donc le point de référence. Le programme fait alors tourner le moteur jusqu'à la détection de l'obturateur "zéro degré". Nous sommes alors certain de notre position de référence dans une marge de plus ou moins un pas du moteur. Le programme assigne alors à cette position la valeur zéro. L'opérateur peut recaler cette position de référence, c'est-à-dire, indiquer au programme qu'il devra considérer cette valeur comme étant un écart autre que zéro.

L'opérateur est alors questionné sur la valeur de l'écart qu'il désire. Le programme prend cette valeur en micromètres et calcule le nombre et la direction des pas que le moteur doit effectuer afin de remplir la condition. Après chaque pas de moteur, le programme relie les valeurs présentes sur le port parallèle et détermine l'état des quatre détecteurs. Si le programme détecte l'activation d'un des interrupteurs de fin de course, il cesse le mouvement du moteur et affiche à l'écran une condition d'erreur "interrupteur de fin de course".

Le programme possède également une routine qui s'effectue après chaque pas du moteur et qui compare la valeur théorique que devrait avoir le détecteur "zéro degré" et sa valeur réelle. En effet nous savons que l'obturateur du détecteur "zéro degré" devrait bloquer la lumière de la cellule photoélectrique à chaque fois que le moteur fait un tour complet. À cause de la largeur de l'obturateur, la lumière devrait rester "bloquée" pour quelque pas du moteur. Si l'état du détecteur ne correspond pas à la valeur qu'il devrait avoir en raison du nombre de pas que nous avons demandé au moteur d'effectuer, nous devons conclure qu'il y a eu problème. Le programme affiche alors un message indiquant que la position actuelle du moteur ne correspond pas à la valeur théorique qu'il devrait avoir. II est alors suggéré à l'opérateur d'effectuer une remise à zéro de la position de la chambre d'ionisation. Il est entendu que cette routine n'assurera pas contre des erreurs lorsque la chambre travaille avec des petits écarts des électrodes, mais il permettra une détection d'erreur à chaque fois que le moteur aura eu à faire un tour complet.

\section{PEEC stepping motor control program}

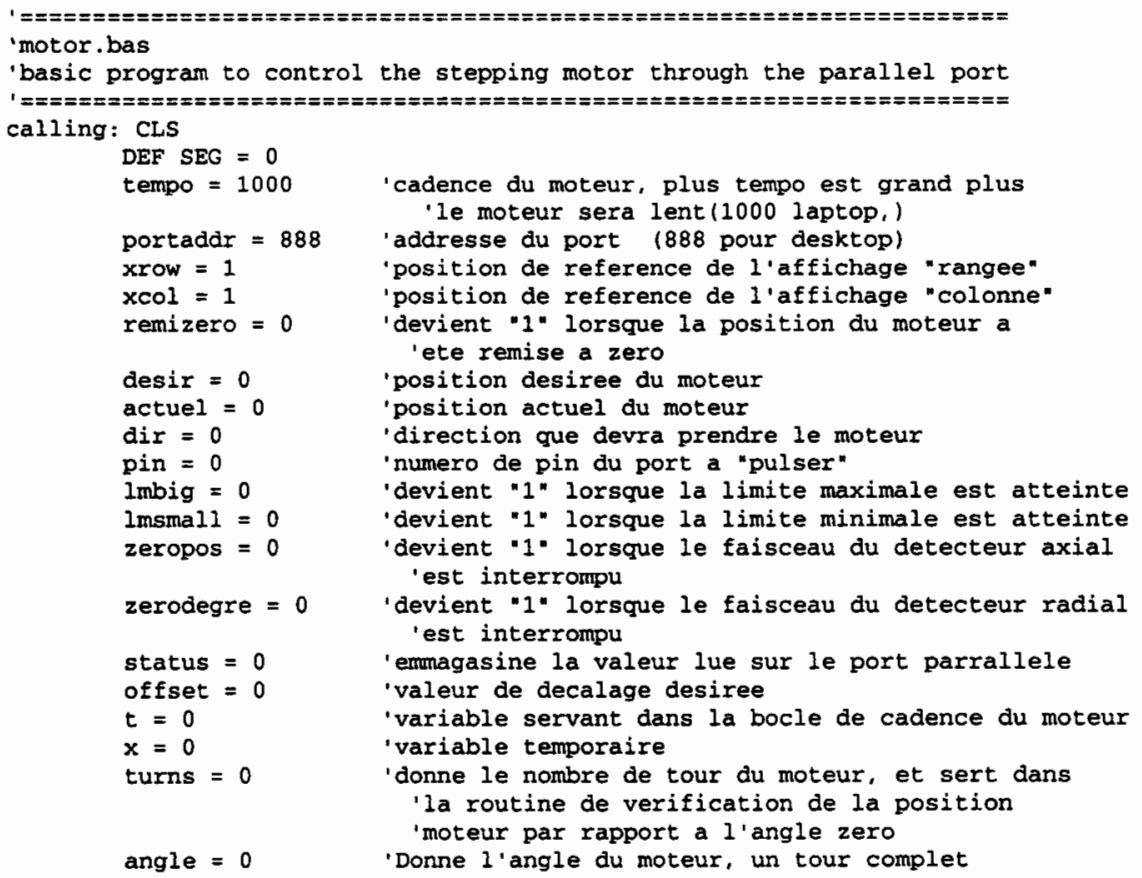




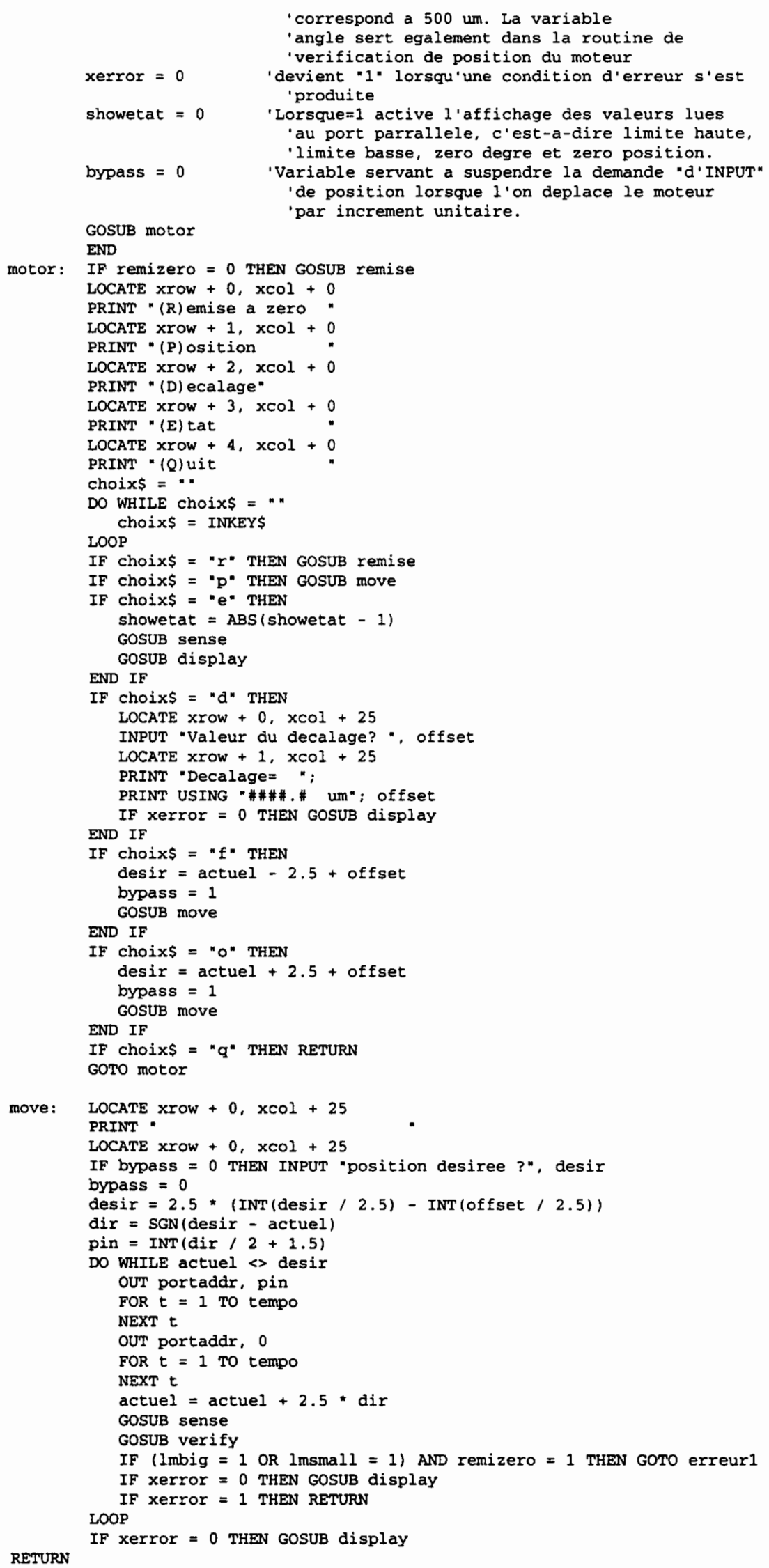




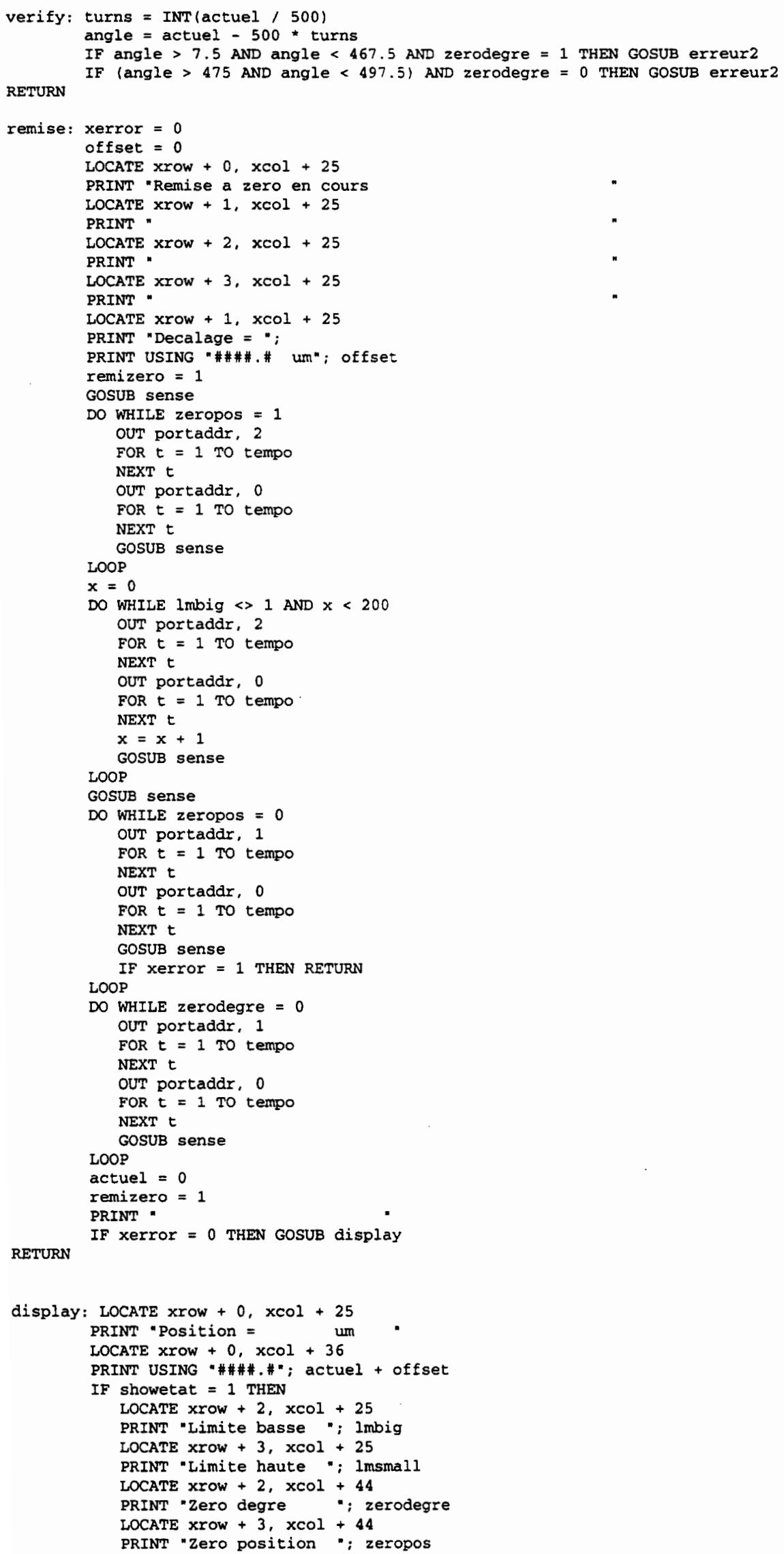


ELSE

LOCATE xrow $+2, x \mathrm{xCl}+25$

PRINT "

LOCATE xrow $+3, x \mathrm{xCO}+25$

PRINT *

LOCATE xrow $+4, x \mathrm{xOl}+25$

PRINT *

LOCATE xrow $+5, x \mathrm{xOl}+25$

PRINT "

END IF

RETURN

sense:

status $=$ INP $($ portadd $x+1)$

lmbig = INT (status $/ 128$ )

temp = INT ( (status - lmbig *128) / 64)

lmsmall = 1 - temp

zerodegre $=\operatorname{INT}($ (status - Imbig $* 128$ - temp *64)/32

RETURN

zeropos = INT ( (status - lmbig* 128 - temp *64 - zerodegre* 32) / 16)

erreur1: LOCATE xrow $+0, x \mathrm{xCO}+25$

PRINT "Une condition anormale a ete rencontree"

LOCATE xrow $+1, x \mathrm{col}+25$

PRINT "Le systeme a rencontre un interrupteur "

LOCATE xrow + 2, xcol + 25

PRINT "de fin de course.

LOCATE xrow +3 , xcol +25

PRINT "Redemarrez le programme

REIURN

xerror $=1$

erreur 2: LOCATE xrow $+0, x \mathrm{xol}+25$

PRINT "La position reelle n'est plus synchronise a la "

LOCATE xrow $+1, x \mathrm{col}+25$

PRINT "position theorique

LOCATE xrow $+2, x \mathrm{xO}+25$

PRINT *

LOCATE xrow $+3, x c 01+25$

PRINT "Redemarrez le programe

RETURN

xerror $=1$ 


\section{Stepping motor schematics}

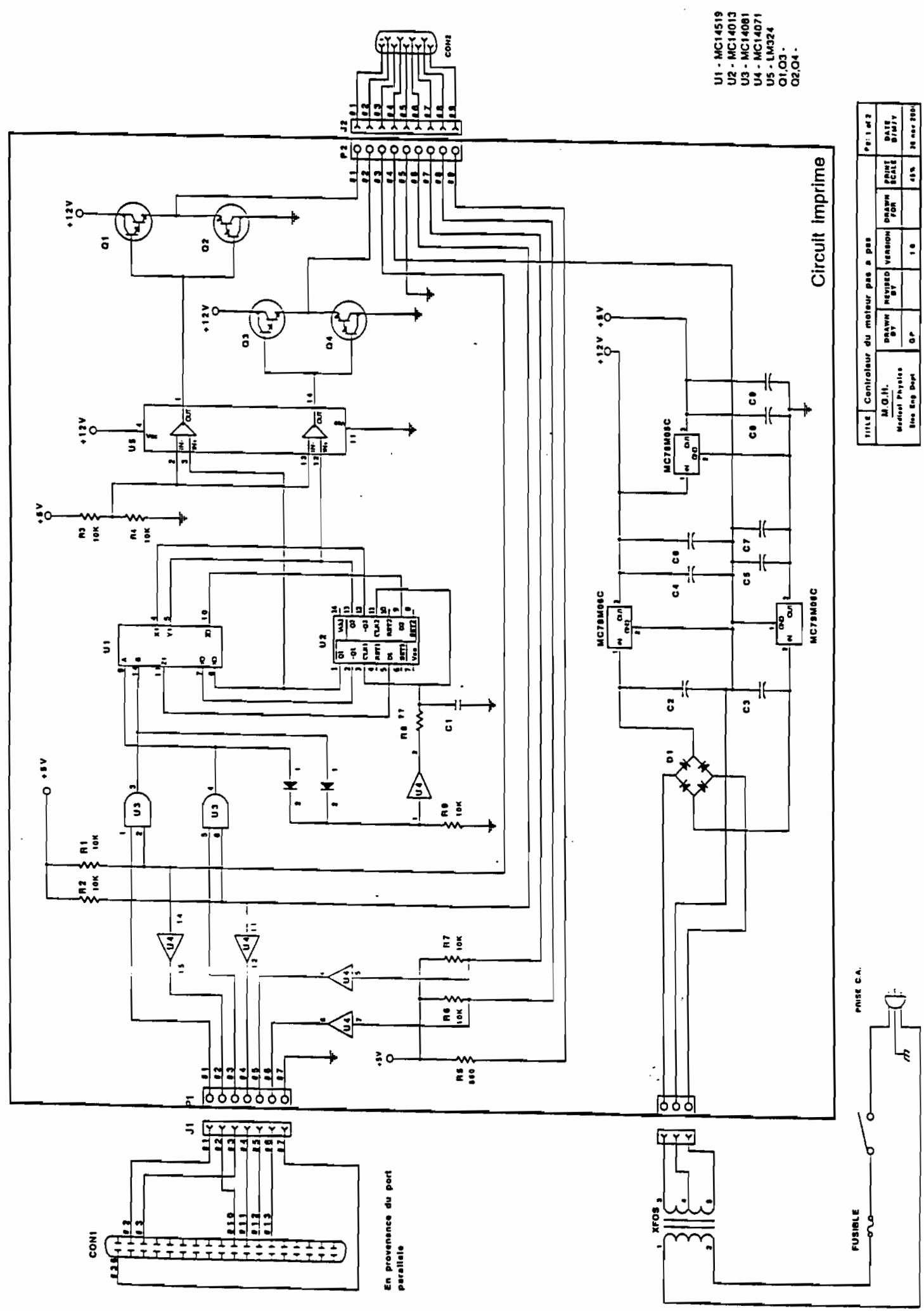




\section{Stepping motor schematics}
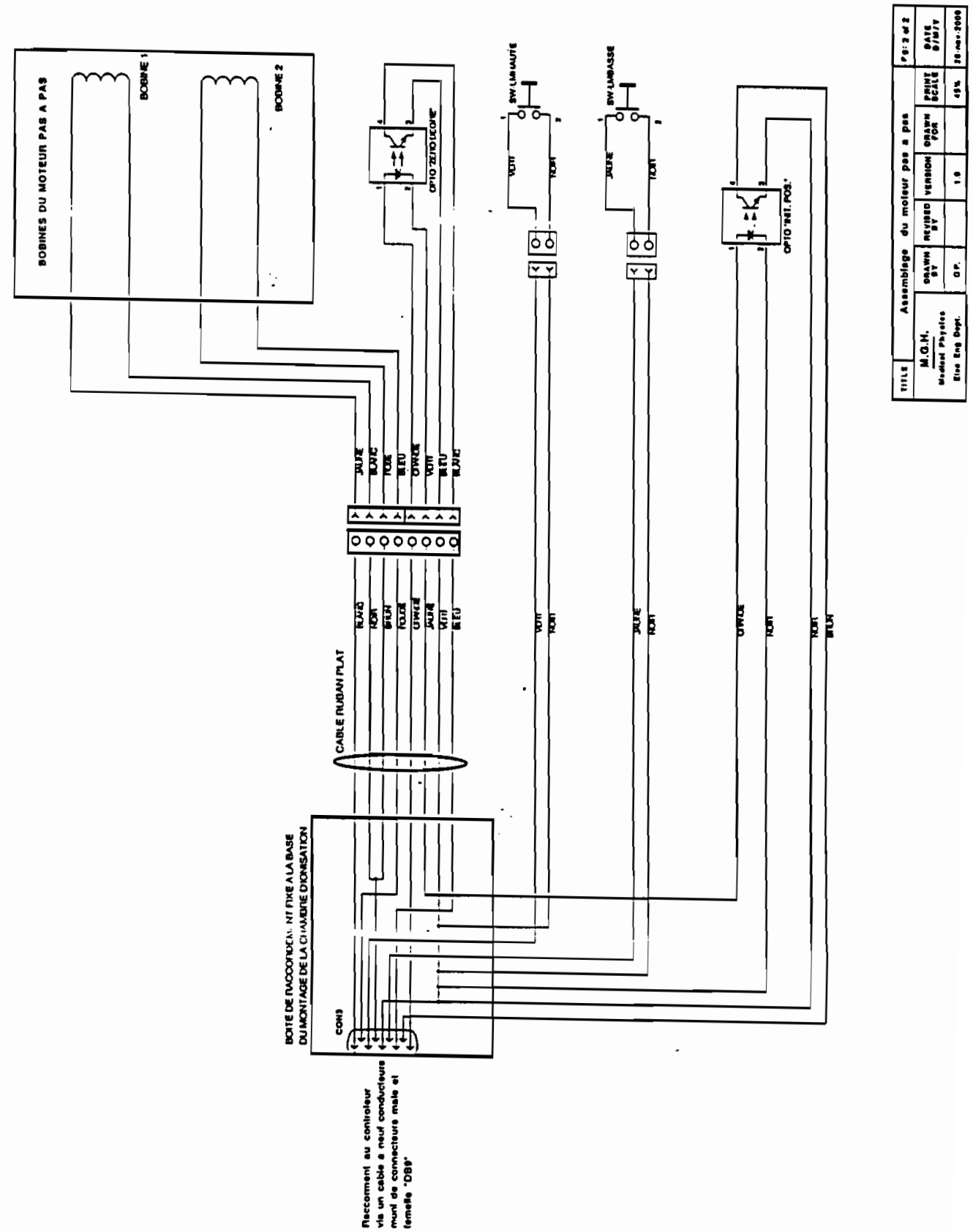
Stepping motor schematics

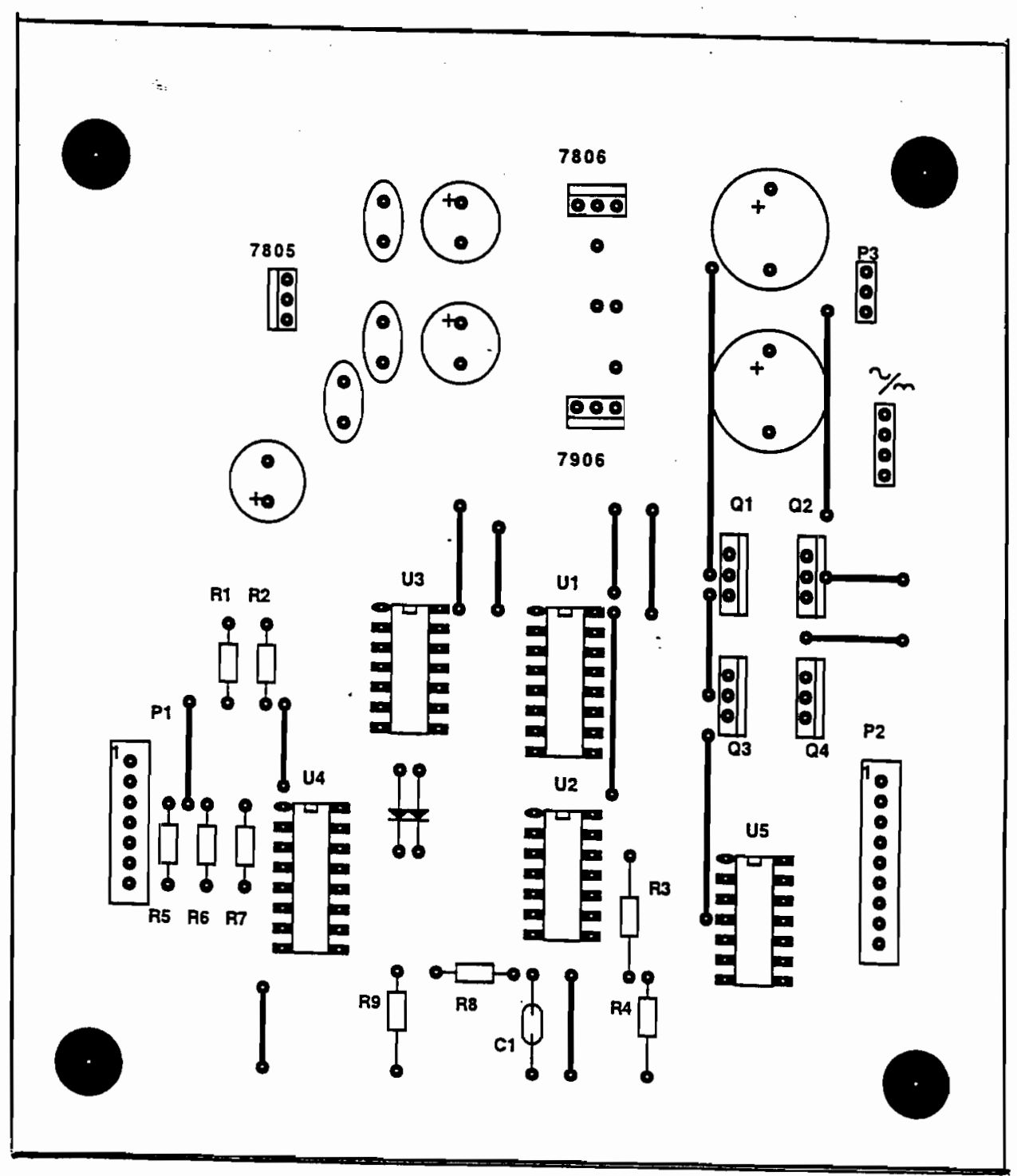

Disposition des

composantes (Vue de dessus) 


\section{Stepping motor schematics}

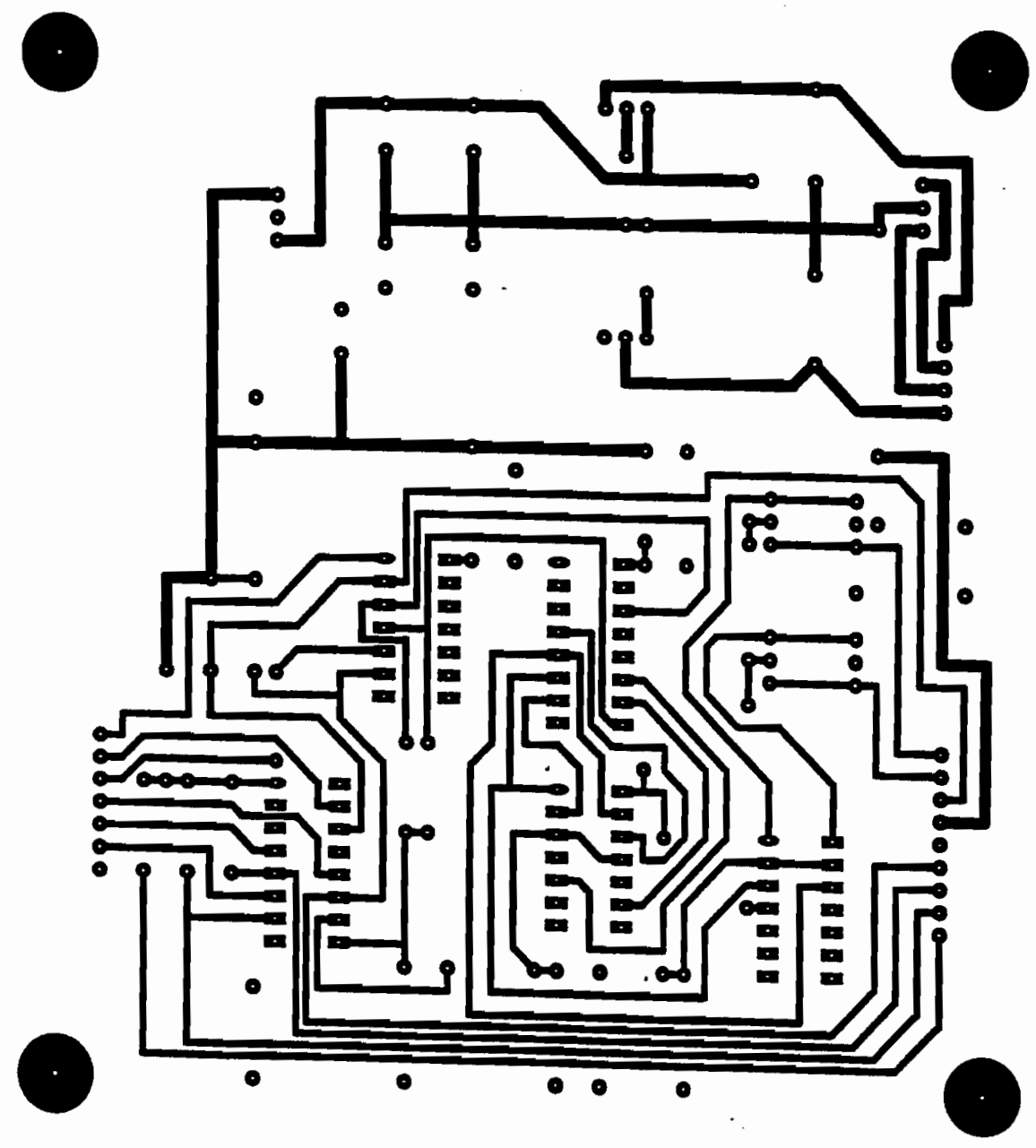

Tracé de cuivre (Vue de dessus)

Excluant la masse 
Stepping motor schematics

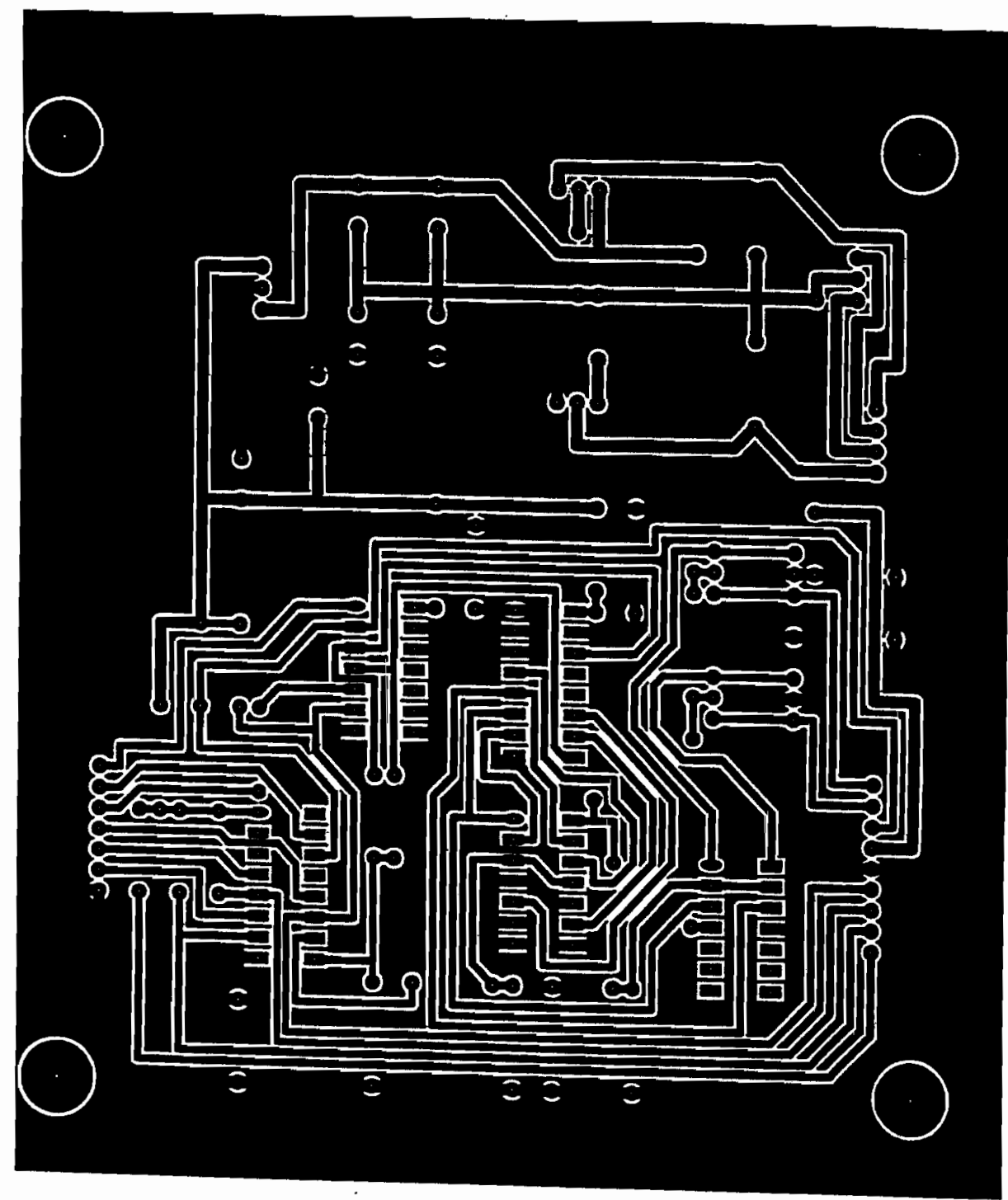

Tracé de cuivre (Vue de dessus) Incluant la masse 


\section{List of Figures}

\section{Chapter 1}

Introduction

Figure 1-1. Classification of radiations as directly and indirectly ionizing radiations. ....................2

Figure 1-2. A medical linear accelerator used for external beam treatment. .......................................

Figure 1-3. (A) Typical photon depth dose curve. (B) Typical electron depth dose curve. Both curves are normalized to a $100 \%$ at $d_{\max }$. .5

Figure 1-4. Typical off-axis ratio curves for various depths $\left(3 \mathrm{~cm}\left(d_{\max }\right), 5 \mathrm{~cm}, 10 \mathrm{~cm}, 15 \mathrm{~cm}, 25\right.$ $\mathrm{cm}$ ) for a $18 \mathrm{MV}$ photon beam with $10 \times 10 \mathrm{~cm} 2$ field size.

Figure 1-5. Schematic diagram of a free-air ionization chamber. The charge collecting volume is $V^{\prime}$ and the ideal volume is $V$ where secondary electron are produced for collection $\left(e_{2}\right)$. The secondary electrons produced in $V$ that escape the collection volume $V^{\prime}\left(e_{3}\right)$ are exactly balanced by the electrons produced outside $V$ that enter $V^{\prime}\left(e_{1}\right)$ for collection.

12

\section{Chapter 2}

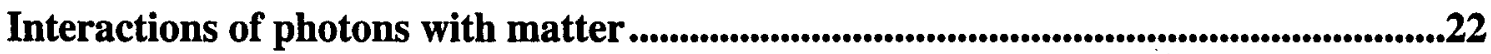

Figure 2-1. Schematic diagram of the photoelectric process. $A$ photon with quantum energy $h v$ interacts with an atomic electron that has a binding energy $E_{b}$. The photon vanishes and the electron is ejected from the atom with a kinetic energy at an angle $\theta$ relative to the incident photon's direction. The atom departs at an angle $\varphi$ in order to conserve momentum. The kinetic energy $T_{\alpha}$ transferred to the above is practically zero because of the relatively large mass of the atom in comparison with the mass of the photoelectron. .25 
Figure 2-2. Schematic diagram of the Compton process. An incident photon with energy $h v$ interacts with a stationary and free electron. The photon is scattered at an angle $\theta$ with an energy $h v$ ' and the electron recoils at an angle $\varphi$ with a kinetic energy $T_{e-}$.

Figure 2-3. Schematic diagram of the pair production process. The incoming photon of energy $h v$ is totally absorbed in the Coulomb field of an atomic nucleus and an electron-positron pair $\left(\mathrm{e}^{+}, \mathrm{e}^{-}\right)$is created. . .28

Figure 2-4. Schematic diagram of the emission of a characteristic photon $K_{\alpha}$ of energy $h v_{l}=E_{K^{-}}$ $E_{L}$ followed by the emission of a characteristic photon $L_{\alpha}$ of energy $h v_{2}=E_{L}-E_{M} \ldots \ldots \ldots$

Figure 2-5. Schematic diagram of a KLM Auger electron emission. An initial vacancy in the Kshell is created, an electron from the L-shell fills the vacancy, and an Auger electron is emitted from the M-shell (KLM Auger electron). The Auger electron is given a total kinetic energy $T_{e}$.

Figure 2-6. Fluorescence yield $\omega$ for the $K$ and $L$ shells. $\omega_{k}$ is calculated from Lederer and Shirley ${ }^{4}$ and $\omega_{L}$ from Burhop 5 .

Figure 2-7. Schematic representation of the cross section, illustrating the difference between the cross section $\sigma$ and the geometrical cross section $\sigma_{g}$

Figure 2-8. The photoelectric atomic cross section for lead illustrating the discontinuities known as the $\mathrm{K}, \mathrm{L}$, and $\mathrm{M}$ absorption edges as well as the subshell discontinuities. .36

Figure 2-9. Compton electronic cross section es calculated with Klein-Nishina formulation (Eq. (216)) and energy transfer coefficient ${ }_{e} \sigma_{t r}$ as a function of incoming photon energy $h v$.

Figure 2-10. Pair production atomic cross section ${ }_{a} k$ as a function of the incident photon energy $h v$ in lead

Figure 2-11. Total mass attenuation coefficient $\mu \rho$ and the partial contribution of the photoeffect $\tau / \rho$, Compton $\sigma / \rho$, and pair-production $\kappa / \rho$ cross-sections for water as a function of photon energy. 
Figure 2-12. Total atomic attenuation coefficients $\mu_{a}$ for three different media: water, copper and lead.

Figure 2-13. Relative importance of the three main photon interactions with matter as a function of the photon energy $h v$ and the atomic number $Z$ of the medium. The solid curves represent the values of $h v$ and $Z$ for which two interactions are of equal importance, i.e., atomic interaction coefficients of effects are equal.

Figure 2-14. Maximum and mean fraction of the kinetic energy given to the recoil electron in a Compton interaction as a function of the incident photon energy $h v$.

\section{Chapter 3}

Interactions of electrons with matter

Figure 3-1. Parameters for an electron collision with atoms. (a) is the classical radius of the atom and (b) is the classical impact parameter. .55

Figure 3-2. Plot of collisional and radiative electron mass stopping power in copper, lead, and water.

Figure 3-3. Sternheimer correction factor $\delta$ for density-effect for electrons in graphite, polystyrene and water as a function of incident electron energy. .63

\section{Chapter 4}

\section{Measurement of absorbed dose}

Figure 4-1. Radiation photon field incident on a sphere of cross sectional area $d A$ around point $P$ for (a) horizontal incidence, and (b) oblique incidence. Particle fluence is the same in (a) and (b), however, the planar fluence decreases for oblique incidence .73

Figure 4-2. Transfer of energy from a photon to the medium. The photon interacts at point $P$, and transfers some of its energy in the form of electron's kinetic energy. The electron, in turn, gives its energy to the medium through small collisions along its track, from $P$ to $P_{\text {end }}($ Ref. \#3). One of the $\delta$ rays carries its energy out of the volume mass $d m$. . .74

Figure 4-3. Illustration of the difference between the kerma and the absorbed dose, without (a) and with (b) attenuation of the photon beam. In (a), the kerma is equal to the dose for 
depths deeper than the depth where electronic equilibrium is reached, whereas in (b) there never is a strict equilibrium and the kerma is always greater than the dose. Collisional kerma is less than total kerma because of energy escaping the volume of interest as bremsstrahlung photons ${ }^{4}$.

Figure 4-4. An electron beam going through an interface between medium $m$ and $c$ of the interface. The fluence is assumed to be identical in the two media, therefore, the absorbed doses on each side are related through Eq. (4-11).

Figure 4-5. A cavity filled with medium $c$ within a homogeneous medium $m$ . .80

Figure 4-6. Basic beam output calibration setup. The point of calibration $P$ is at depth $d_{\max }$ in a water phantom that is irradiated by a $10 \times 10 \mathrm{~cm}^{2}$ field produced by a source located at a distance $S S D$ from the surface of the phantom. .88

Figure 4-7. Schematic diagram of an ionization chamber circuitry for a typical radiation dosimetry system.

\section{Chapter 5}

Experimental apparatus and techniques .108

Figure 5-1. Schematic representation of the PEEC. The Solid Water ${ }^{\mathrm{TM}}$ material is shown in light grey and the removable parts, entrance window and piston, are shown in darker grey.

Figure 5-2. Photograph of the PEEC with the bone entrance window installed. The Lucite frame and the high voltage, signal and stepping motor connectors are also shown.

Figure 5-3. The entrance window with the polarizing electrode holder.

Figure 5-4. Schematic diagram of the PEEC computer control system. .115

Figure 5-5. Computer system to remotely control the PEEC: a 486 personal computer controls the electrometer (Keithley 35617) the high voltage power supply (Keithley 248) on the IEEE-488 interface and the stepping motor interface through the parallel port. 
Figure 5-6. Relative position of the micrometer as a function of the software requested position. The agreement is excellent except for the first point at which the micrometer is positioned at its lower limits.

Figure 5-7. Inverse capacitance as a function of relative electrode separation measured with the extrapolation chamber with the bone material in place. The slope of the least-squares fit to measured data is $\left(\varepsilon_{0} A\right)^{-1}$ from which the effective collecting electrode area $A$ is calculated

Figure 5-8. Saturation charge as a function of the relative electrode separation measured with the extrapolation chamber for a $9 \mathrm{MeV}$ electron beam and the bone piston top and entrance window.

Figure 5-9. Plot of the residuals between $C$ and $C^{\prime}$.

Figure 5-10. PEEC chamber leakage current as a function of polarizing voltage.

\section{Chapter 6}

Monte Carlo calculations

Figure 6-1. EGS program flow chart separating user code from core code (from Bielajew ${ }^{33}$ )

Figure 6-2. Flow chart of simulation process for a radiotherapy treatment machine.

Figure 6-3. Treatment heads of medical linear accelerators in photon mode (A) and in electron mode (B). In photon mode, an $x$-ray target and a flattening filter are used and in the electron mode a scattering foil and an electron applicator are required.

Figure 6-4. 3-D view of the Clinac 18 treatment machine head in electron mode 143

Figure 6-5. Percent depth doses measured with RFA and Roos chamber (circle dots) and calculated with DOSRZnrc (lines) in Water for $9 \mathrm{MeV}$ (A) and for $15 \mathrm{MeV}$ (B) beams with $10 \times 10$ $\mathrm{cm}^{2}$ applicators. 
Figure 6-6. Profiles measured with RFA and electron diodes (dots) and calculated with DOSXYZ (lines) in water for $9 \mathrm{MeV}$ beam with $10 \times 10 \mathrm{~cm}^{2}$ applicator. .145

Figure 6-7. Percent depth doses measured with TLDs (dots) and calculated with DOSRZnrc (lines) in various materials for a $9 \mathrm{MeV} 15 \times 15 \mathrm{~cm}^{2}$ beam without electron applicator. Values are normalized to $100 \%$ to $d_{\max }$ in Solid Water ${ }^{\mathrm{TM}}$ (black square points).

Figure 6-8. Percent depth doses measured with TLDs (dots) and calculated with DOSRZnrc (lines) in various materials for a $15 \mathrm{MeV} 15 \times 15 \mathrm{~cm}^{2}$ beam without electron applicator. Values are normalized to $100 \%$ to $d_{\max }$ in Solid Water ${ }^{\mathrm{TM}}$ (black square points).

Figure 6-9. 3-D representation of the Clinac $2300 \mathrm{C} / \mathrm{D}$ treatment head components in photon mode. The location of the phase-space is shown in (A) and a zoom view on the component is shown in (B) with some particles histories represented by lines.

Figure 6-10. Percent depth dose curves for 6 and $18 \mathrm{MV} 10 \times 10 \mathrm{~cm}^{2}$ fields, $S S D=100 \mathrm{~cm}$, in Solid Water ${ }^{\mathrm{TM}}$ and bone equivalent material, measured with PEEC (square dots) and calculated with DOSRZnrc (lines). .148

Figure 6-11. Off-axis profiles for the Clinac-2300 C/D $6 \mathrm{MV}$ beam $10 \times 10 \mathrm{~cm} 2$ measured with RFA and RK chambers and calculated with DOSXYZ (lines) in water.

Figure 6-12. Off-axis profiles for the Clinac-2300 C/D $18 \mathrm{MV}$ beam $10 \times 10 \mathrm{~cm}^{2}$ measured with RFA and RK ion chambers (dots) and calculated with DOSXYZ (lines) in water.

Figure 6-13. A side view (A) and a 3-D (B) representation of the T-780 treatment machine head........ .150

Figure 6-14. Percent depth dose curves for Solid Water ${ }^{\mathrm{TM}}$ and bone equivalent material measured with PEEC (circles and squares) and calculated with Monte Carlo (lines). 
Figure 6-15. (a) Electron spectrum (Clinac-18) for a $9 \mathrm{MeV}$ electron beam ( $S S D=100 \mathrm{~cm}$, field size $=10 \times 10 \mathrm{~cm}^{2}$ ) with (b) associated photon contamination spectrum. The spectra were calculated with Monte Carlo technique.

Figure 6-16. (a) Electron spectrum (Clinac-18) for a $15 \mathrm{MeV}$ electron beam $(S S D=100 \mathrm{~cm}$, field size $=10 \times 10 \mathrm{~cm}^{2}$ ) with (b) associated photon contamination spectrum. The spectra were calculated with Monte Carlo technique.

Figure 6-17. Photon spectrum (Clinac-2300 C/D) for a $6 \mathrm{MV}$ photon beam $(S S D=100 \mathrm{~cm}$, field size $=10 \times 10 \mathrm{~cm}^{2}$ ). The spectrum was calculated with Monte Carlo technique.

Figure 6-18. Photon spectrum (Clinac-2300 C/D) for a $18 \mathrm{MV}$ photon beam $(S S D=100 \mathrm{~cm}$, field size $\left.=10 \times 10 \mathrm{~cm}^{2}\right)$. The spectrum was calculated with Monte Carlo technique.

Figure 6-19. Photon spectrum (T-780) for a cobalt-60 photon beam $(S S D=80 \mathrm{~cm}$, field size $=10 \times 10 \mathrm{~cm}^{2}$ ). The spectrum was calculated with Monte Carlo technique.

Figure 6-20. The simulated model of the PEEC in a right cylindrical geometry $(\rho, z)$ used for Monte Carlo with DOSRZnrc. The chamber is assumed to be cylindrical. The drawing is not to scale. .155

\section{Chapter 7}

\section{Collection efficiency for ionization chambers in pulsed beams}

Figure 7-1. Saturation data measured with an ionization chamber (PR-06C) in an $18 \mathrm{MV}$ pulsed photon beam at $\sim 400 \mathrm{cGy} / \mathrm{min}$. Part (a) shows data in the polarizing voltage region from $\sim 10^{\circ}$ to $\sim 1050 \mathrm{~V}$, with the solid line encompassing points used in linear extrapolation to $1 / V=0$. Part (b) shows the shaded region of part (a) on an expanded scale, with the solid line representing the linear extrapolation of data shown in part (a), the dashed line representing the "two-voltage" technique through 150 and $300 \mathrm{~V}$ points, and the dotted curve representing Eq. (7-7) with $\lambda_{p}=1.31 \mathrm{~V} / \mathrm{nA}$ and $\gamma=$ $1.37 \times 10^{-5} \mathrm{~V}^{-1}$ 
Figure 7-2. Saturation data measured with an ionization chamber (PR-06C) in an $18 \mathrm{MV}$ pulsed photon beam at various dose rates in the polarizing voltage region from $\sim 10$ to $\sim 1050 \mathrm{~V}$. The graphs on the right represent data from the shaded region on the left. The dotted curves represent Eq. (7-7) with appropriate parameters $\lambda_{p}$ and $\gamma$ given in Table 7-1; the dashed lines represent the "two-voltage" technique; and the solid lines represent linear extrapolation of data in the region $0.90<f<0.98$ to $1 / V=0 \ldots . .172$

Figure 7-3. Saturation data measured with an ionization chamber (PR-06C) in pulsed 6 and $18 \mathrm{MV}$ photon beams and 9 and $18 \mathrm{MeV}$ electron beams at $\sim 400 \mathrm{cGy} / \mathrm{min}$ in the polarizing voltage region from $\sim 10$ to $\sim 1050 \mathrm{~V}$. The graphs on the right represent data from the shaded region on the left. The dotted curves represent Eq. (7-7) with parameters $\lambda_{p}$ and $\gamma$ given in Table 7-1; the dashed lines represent the "two-voltage" technique; and the solid lines represent linear extrapolation of data in the region $0.90<f<0.98$ to $1 / V=0$ .173

Figure 7-4. Schematic view of the three irradiation fields showing the position of the chamber, the field, and the shielding for measurements with the PR-06C ionization chamber. Part (a) is for the $10 \times 10$ and $30 \times 30 \mathrm{~cm}^{2}$ open fields, part (b) for the $10 \times 10 \mathrm{~cm}^{2}$ field with a shielded stem, connector and cable of the ionization chamber.

Figure 7-5. Saturation data measured with an ionization chamber (PR-06C) in an $18 \mathrm{MV}$ pulsed photon beam at $\sim 400 \mathrm{cGy} / \mathrm{min}$ in the polarizing voltage range from $\sim 20$ to $\sim 1050 \mathrm{~V}$ with the three field arrangements shown in Fig. . The solid lines represent linear fits to the linear portion of the data; the dashed lines the fit of the data to Eq. (7-7); and the dotted lines the "two-voltage" technique. b) Saturation data measured with an ionization chamber (NE2571) in an $18 \mathrm{MV}$ pulsed photon beam at $400 \mathrm{MU} / \mathrm{min}$ in the polarizing voltage range from $\sim 20$ to $\sim 700 \mathrm{~V}$ for the two field sizes: $10 \times 10 \mathrm{~cm}^{2}$ and $30 \times 30 \mathrm{~cm}^{2}$. The solid lines are the linear fits to the linear portion of the data, the dashed lines are the fit of the data to Eq. (7-7). The position of the 150 and $300 \mathrm{~V}$ points are indicated with the two vertical dashed lines.

Figure 7-6. Saturation data measured with a Holt parallel plate ionization chamber in an $18 \mathrm{MV}$ pulsed photon beam at $\sim 400 \mathrm{cGy} / \mathrm{min}$ in the polarizing voltage region from 8 to $900 \mathrm{~V}$ for two fields: $3 \times 3 \mathrm{~cm}^{2}$ and $15 \times 15 \mathrm{~cm}^{2}$. The solid lines represent the linear fit to the linear portion of the data, the dashed lines the "two-voltage" technique. .180 
Figure 7-7. Saturation data measured with the Solid Water ${ }^{\mathrm{TM}}$ PEEC parallel plate ionization chamber (with $2.5 \mathrm{~mm}$ electrode spacing) in an $18 \mathrm{MV}$ pulsed photon beam at $\sim 400$ cGy/min in the polarizing voltage region from 50 to $800 \mathrm{~V}$ for two fields: $5 \times 5 \mathrm{~cm}^{2}$ and $10 \times 10 \mathrm{~cm}^{2}$. The dashed lines represent the linear fit to the linear portion of the data which is identical to the "two-voltage" technique result. The solid lines represent the fit to Eq. (7-7).

\section{Chapter 8}

Measurement of absorbed dose in bone-equivalent material 186

Figure 8-1. The simulation setup for scatter analysis of a bone-equivalent phantom: (a) hybrid chamber resulting from modification of the SW original chamber and (b) PEEC chamber built entirely from bone material.

Figure 8-2. Air cavity perturbation factor for $9 \mathrm{MeV}$ for the Solid Water ${ }^{\mathrm{TM}}$ PEEC.

\section{Chapter 9}

Conclusions 


\section{List of Tables}

\section{Chapter 1}

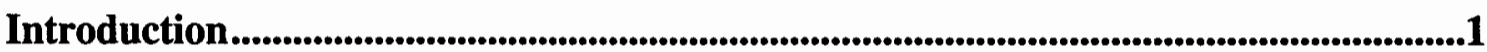

\section{Chapter 2}

Interactions of photons with matter ...................................................................................22

Table 2-1. Dependence of the photoelectric, Compton and pair production processes on $h v$ and

$Z$. The electronic and atomic cross section and the linear and mass attenuation coefficient dependence is shown.

\section{Chapter 3}

Interactions of electrons with matter

\section{Chapter 4}

Measurement of absorbed dose

\section{Chapter 5}

Experimental apparatus and techniques

Table 5-1. Tissue-equivalent plastic and electrode material properties. $Z_{\text {eff }}$ is calculated from the formula-given in Johns ${ }^{12}$.

\section{Chapter 6}




\section{Chapter 7}

Collection efficiency for ionization chambers in pulsed beams 162

Table 7-1. Parameters of saturation curves measured with a cylindrical Farmer-type ionization chamber (PR-06) in 6 and $18 \mathrm{MV}$ pulsed photon beams and in $9 \mathrm{MeV}$ and $18 \mathrm{MeV}$ pulsed electron beams (all field sizes are $10 \times 10 \mathrm{~cm}^{2}$ ). Column 3 is obtained by a linear fit to the data, Column 4 is obtained by Eq. (7-6) and Column 5 is obtained by Eq. (7-7). Column 9 is found by $100 \frac{(\operatorname{col}(3)-\operatorname{col}(5))}{\operatorname{col}(5)}$ and Column 10 is found by $100 \frac{(\operatorname{col}(4)-\operatorname{col}(5))}{\operatorname{col}(5)}$

Table 7-2. Parameters of saturation curves measured with ionization chambers (PR-06, NE2571, Holt and PEEC chamber) in $18 \mathrm{MV}$ pulsed photon beams at a dose rate of $\sim 400 \mathrm{cGy} / \mathrm{min}$ .178

\section{Chapter 8}

\section{Measurement of absorbed dose in bone-equivalent material}

Table 8-1. Results of absolute calibration in bone-equivalent material carried out with a hybrid PEEC using graphite electrodes. Dose is calculated with Eq. (8-3), $\rho=1.293 \times 10^{-3} \mathrm{~g} /$ $\mathrm{cm}^{3}, A=8.019 \mathrm{~cm}^{2}, \bar{W}_{\text {air }}=33.97 \mathrm{~J} / \mathrm{C} .(\bar{L} / \rho)_{\text {air }}^{\text {bone }}$ is calculated with SPRRZnrc for each beam; $d Q / d z$ is measured at depth $d_{\max }$ for electron beams and at a depth of 6 $\mathrm{cm}$ for photon beams; ionization readings are corrected with the two voltage technique for ionic recombination and are also corrected for temperature, pressure and polarity effect. Dose (in cGy) is given at $d_{\max }$ (through appropriate percentage depth doses) for an exposure time of 1 minute for Co-60 (SSD = $80 \mathrm{~cm}$ ) and $100 \mathrm{cGy}$ in water for $10 \times 10 \mathrm{~cm}^{2}$ linac $(S S D=100 \mathrm{~cm}$ ) beams. The graphite electrodes are $0.005 \mathrm{~cm}$ thick and a $2 \mathrm{~cm}$ bone thickness on the back of the collecting electrode is used....... .193

Table 8-2. Monte Carlo calculated ratios of dose-to-air in the chamber cavity for various thicknesses $t(1,2$, and $10 \mathrm{~cm})$ of bone material below the collecting electrode for the hybrid bone PEEC. The air cavity ( $2 \mathrm{~mm}$ thick) is at depth $d_{\max }$ for electron beams and at a depth of $6 \mathrm{~cm}$ for photon beams, field size $10 \times 10 \mathrm{~cm}^{2}, S S D 80 \mathrm{~cm}$ for cobalt-60 beam and SSD $100 \mathrm{~cm}$ for linacs beams 
Table 8-3. Monte Carlo calculated ratios of air cavity dose for SW PEEC with and without $0.005 \mathrm{~cm}$ thick graphite electrodes. The air cavity ( $2 \mathrm{~mm}$ thick) is at depth $d_{\max }$ for electron beams and at a depth of $6 \mathrm{~cm}$ for photon beams, field size $10 \times 10 \mathrm{~cm}^{2}, S S D$ $80 \mathrm{~cm}$ for cobalt-60 beam and SSD $100 \mathrm{~cm}$ for linacs beams. .201

Table 8-4. Percent difference between dose to air cavity with no electrode and with specific electrode combinations for photon beams. Graphite and aluminum electrodes are $0.005 \mathrm{~cm}$ thick, steel and brass electrodes are $0.0025 \mathrm{~cm}$ thick, for a $2 \mathrm{~cm}$ bone piston material on the back of the collecting electrode. The data were calculated with Monte Carlo techniques. The air cavity ( $2 \mathrm{~mm}$ thick) is at depth $6 \mathrm{~cm}$, field size $10 \times 10 \mathrm{~cm}^{2}, S S D 80 \mathrm{~cm}$ for cobalt-60 beam and $S S D 100 \mathrm{~cm}$ for linacs beams.

Table 8-5. Percent difference between dose to air cavity with no electrode and with specific electrode combinations for electron beams. Graphite and aluminum electrodes are $0.005 \mathrm{~cm}$ thick, steel and brass electrodes are $0.0025 \mathrm{~cm}$ thick, for a $2 \mathrm{~cm}$ bone piston material on the back of the collecting electrode. The data were calculated with Monte Carlo techniques. The air cavity ( $2 \mathrm{~mm}$ thick) is at depth $d_{\max }$, field size $10 \times 10 \mathrm{~cm}^{2}$, and $S S D 100 \mathrm{~cm}$ .204

Table 8-6. Comparison of dose-to-air ratios calculated with Monte Carlo and measured with bone PEEC for various electrode materials. The air cavity dose is given at depth $d_{\max }$ for electron beams and at a depth of $6 \mathrm{~cm}$ for photon beams, for $100 \mathrm{MU}$, field size $10 \times 10 \mathrm{~cm}^{2}, S S D 100 \mathrm{~cm}$. Ratios are normalized to dose to air for the graphite/ graphite dose. The air cavity thickness is varied from 2 to $3 \mathrm{~mm}$ for experimental data and is fixed at $2 \mathrm{~mm}$ for calculated data .205

Table 8-7. Dose to the chamber air cavity calculated with Monte Carlo techniques as a function of graphite electrode thickness for $18 \mathrm{MV}$ beam. All values are normalized to the $0.0025 \mathrm{~cm}$ values. The air cavity is at a depth of $6 \mathrm{~cm}$, field size $10 \times 10 \mathrm{~cm}^{2}, S S D 100$ $\mathrm{cm}$. The air cavity thickness is fixed at $2 \mathrm{~mm}$ .206

Table 8-8. Dose correction factor $C_{c h}$ calculated with Monte Carlo techniques for bone PEEC at depth $d_{\max }$ for electron beams and at depth $6 \mathrm{~cm}$ for photon beams, field size $10 \times 10 \mathrm{~cm}^{2}, S S D 80 \mathrm{~cm}$ for cobalt $-60, S S D 100 \mathrm{~cm}$ for linacs beams. $C_{c h}$ also includes correction for humidity $k_{h}=0.997$. 
Table 8-9. Results of absolute calibration in bone-equivalent material carried out with bone PEEC using graphite electrodes. Dose is calculated with Eq. (8-4), $\rho=1.293 \times 10^{-3}$ $\mathrm{g} / \mathrm{cm}^{3}, A=8.019 \mathrm{~cm}^{2}, \bar{W}_{\text {air }}=33.97 \mathrm{~J} / \mathrm{C} .(\bar{L} / \rho)_{\text {air }}^{\text {bone }}$ is calculated with SPRRZnrc for each beam; $d Q / d z$ is measured at depth $d_{\max }$ for electron beams and at a depth of $6 \mathrm{~cm}$ for photon beams; ionization readings are corrected with the two voltage technique for ionic recombination and are also corrected for temperature, pressure and polarity effect. Dose (in cGy) is given at $d_{\max }$ (through appropriate percentage depth doses) for an exposure time of 1 minute for Co-60 (SSD = $80 \mathrm{~cm})$ and $100 \mathrm{cGy}$ in water for $10 \times 10 \mathrm{~cm}^{2}$ linac (SSD $=100 \mathrm{~cm}$ ) beams. The graphite electrodes are $0.005 \mathrm{~cm}$ thick and a $2 \mathrm{~cm}$ bone thickness on the back of the collecting electrode is used. $D_{b o n e}$ is corrected with $C_{c h}$ which includes corrections for lack of scatter and for graphite electrode perturbation as well as humidity correction. .208

\section{Chapter 9}




\section{Bibliography}

AAPM Task Group 21 of the Radiation Therapy Committee, "A protocol for the determination of absorbed dose from high-energy photon and electron beams," Med. Phys. 10, 741-771 (1983). $(11,88,112,165,165,187,198)$

Task group 25 American Association of Physicist in Medicine, "Clinical electron beam dosimetry," Med. Phys. 18, 73-109 (1991).

P. R. Almond, P. J. Biggs, B. M. Coursey et al., "AAPM's TG-51 protocol for clinical reference dosimetry of high-energy photon and electron beams," Med. Phys. 26 (9), $1847-1870$ (1999). $(11,88,100,112,187,191,192)$

F. H. Attix, "A simple derivation of Ngas, a correction in Awall, and other comments on the AAPM Task Group 21 protocol," Med. Phys. 11 (5), 725-728 (1984).

Frank H. Attix, Introduction to radiological physics and radiation dosimetry (Wiley \& Son, New York, 1986). .$(9,10,31,36,37,39,48,49,57,64)$

M. J. Berger, "Monte Carlo Calculation of the penetration and diffusion of fast charged particles," in Methods in Comput. Phys., edited by B. Alder, S. Fernbach and M. Rotenberg (Academic, New York, 1963), Vol. 1, pp. 135-215.

M. J. Berger and S. M. Seltzer, ETRAN, Monte Carlo code system for electron and photon transport through extended media, RISC computer code, package CCC-107 (Oak Ridge National Laboratory, Oak Ridge, TN, 1973). 
M. J. Berger and S. M. Seltzer, "Stopping powers and ranges of electrons and positrons," Report NBSIR 82-2550-A, National Bureau of Standards, Washington D. C. (1983). $(62,64,133,191)$

M. J. Berger and R. Wang, "Multiple-Scattering Angular Deflections and Energy-Loss Straggling.," in Monte Carlo Transport of Electrons and Photons, edited by Theodore M. Jenkins, Walter R. Nelson and Alessandro Rindi (New York, 1988), pp. 21-56.

H. A. Bethe, "Zur Theorie des Durchgangs schneller Korpuskularstrahlen durch Materie," Ann. Physik 5, 325 (1930). $(57,61,62)$

H. Bethe and W. Heitler, "On the stopping of fast particles and on the creation of positive electrons," Proc. Roy. Soc. A. 146, 83-112 (1934). $(39,57,61,62)$

A. F. Bielajew, H. Hirayama, W. R. Nelson et al., "History, overview and recent improvements of EGS4," Technical Report PIRS-0436, National Research Concil of Canada, Ottawa, Canada (1994)

M. D. Blaufox, "Becquerel and the discovery of radioactivity: early concepts," Semin. Nucl. Med. 26 (3), 145-154 (1996).

J. W. Boag, "Ionization chambers," in The dosimetry of ionizing radiation, edited by K. R. Kase, B. E. Bjärngard and F. H. Attix (Academic Press, Orlando, 1985), Vol. 2, pp. 169-243. $(95,123,162)$

J. W. Boag, "The saturation curve for ionization measurements in pulsed radiation beams," Brit. J. Radiol. 25, 649-650 (1952). 
J. W. Boag, "Ionization chambers," in Radiation dosimetry, edited by F. H. Attix, W. C. Roesch and E. Tochilin (Academic Press, New York, 1966), Vol. 2, pp. 1-72.

J. W. Boag, "Ionization measurements at very high intensities I. Pulsed radiation beams," Br. J. Radiol. 23, 601-611 (1950).

J. W. Boag, "The saturation curve for ionization measurements in pulsed radiation beams," Brit. J. Radiol. 25, 649-650 (1952).

J. W. Boag and J. Currant, "Current collection and ionic recombination in small cylindrical ionization chambers exposed to pulsed radiation," Br. J. Appl. Phys. 53, 471-478 (1980).

J. W. Boag, E. Hochhauser, and O. A. Balk, "The Effect of Free-Electron Collection On the Recombination Correction to Ionization Measurements of Pulsed Radiation," Phys. Med. Biol. 41 (5), 885-897 (1996).

J. Böhm and U. Schneider, "Review of extrapolation chamber measurements of beta rays and low energy x rays," Radiat. Prot. Dosim. 14, 193-198 (1986).

N. Bohr, "On the Theory of the Decrease of Velocity of Moving Electrified Particles on Passing through Matter," Phil. Mag. 25, 10 (1913).

N. Bohr, “Kgl. Danske Vịdenskab. Selskab,” Mat.-Fys. Medd. 18 (8, 1948). (57)

M. Boutillon and A. M. Perroche-Roux, "Re-evaluation of the W value for electrons in dry air," Phys. Med. Biol. 32, 213-219 (1987). $(11,190)$

W. H. Bragg, "Consequences of the corpuscular hypothesis of the gamma and $\mathrm{x}$ rays, and the ranges of beta rays," Phil. Mag. 20, 385 (1910). 
Judith F. Briesmeister, "MCNP - A General Monte Carlo N-Particle Transport Code," Report LA-13709-M, Los Alamos National Laboratory, Los Alamos (2000).

E. H. S. Burhop, The Auger effect and other radiationless transitions (University Press, Cambridge, 1952).

T. E. Burlin, "The measurement of exposure dose for high energy radiation with cavity ionization chambers," Phys. Med. Biol. 3, 197-206 (1959).

J. C. Butcher and H. Messel, "Electron number distribution in electron-photon showers," Phys. Rev. 112, 2096-2106 (1958).

Chemical Rubber Company, CRC Handbook of Chemistry and Physics, 78th ed. (Chemical Rubber Pub. Co., Cleveland, 1997).

C. Constantinou, F. H. Attix, and B. R. Paliwal, "A solid water phantom material for radiotherapy x-ray and gamma-ray beam calibrations," Med. Phys. 9 (3), 436-441 (1982).

C. Constantinou, N. F. Kember, G. Huxtable et al., "Physical measurements with a high-energy proton beam using liquid and solid tissue substitutes," Phys. Med. Biol. 25 (3), 489-499 (1980).

G. Comte de Buffon, "Essai d'arithmétique morale," in volume 4. Supplément à l'Histoire Naturelle (1777).

F. DeBlois, C. E. Zankowski, and E. B. Podgorsak, "Saturation current and collection efficiency for ionization chambers in pulsed beams," Med. Phys. 27 (5), 1146-1155 (2000). 
K. Derikum and M. Roos, "Measurement of saturation correction factors of thimble-type ionization chambers in pulsed photon beams," Phys. Med. Biol. 38 (6), 755-763 (1993).

G. X. Ding, D. W. Rogers, and T. R. Mackie, "Calculation of stopping-power ratios using realistic clinical electron beams," Med. Phys. 22 (5), 489-501. (1995).

G. X. Ding and D. W. Rogers, "Mean energy, energy-range relationships and depth-scaling factors for clinical electron beams," Med. Phys. 23 (3), 361-376. (1996).

J. Dutreix, "From X-rays to radioactivity and radium. The discovery and works of Henri Becquerel (1851-1908)," Bull. Acad. Natl. Med. 180 (1), 109-118 (1996).

J. Dutreix and A. Dutreix, "Henri Becquerel (1852-1908)," Med. Phys. 22 (11 Pt 2), 1869-1875 (1995).

J. R. Ehrman, "The care and feeding of random numbers," SLAC VM Notebook, Module 19, SLAC Computing Services (1981).

Robley Dunglison Evans, The atomic nucleus (R.E. Krieger, Malabar, Florida, 1982).

G. Failla, "Measurement of tissue dose in terms of the same unit for all ionizing radiations," Radiology 29, 202-215 (1937).

R. L. Ford and W. R. Nelson, "The EGS Code system - Version 3," Stanford Linear Accelerator Center Report SLAC-210 (Stanford Calif., 1978).

Gammex RMI (Private communications). 
S. Genna and J. S. Laughlin, "Absolute calibration of a cobalt-60 gamma-ray beam," Radiology 65, 394-405 (1955).

Otto Glasser and Wilhelm Conrad Röntgen, Dr. W. C. Röntgen (C. C. Thomas, Springfield, Ill., 1945).

Otto Glasser, Jessie C. Tucker, and Margret Boveri, Wilhelm Conrad Röntgen and the early history of the Roentgen rays (Bale \& Danielsson, London, 1933).

M. L. Goldberger, "The interaction of high energy neutrons and heavy nuclei," Phys. Rev. 74, 1269-1277 (1948).

S. Goudsmit and J. L. Saunderson, "Multiple Scattering of Electrons," Phys. Rev. 57, 24-29 (1940).

S. Goudsmit and J. L. Saunderson, "Multiple Scattering of Electrons II," Phys. Rev. 58, 36-42 (1940).

L.H. Gray, "Absorption of penetrating radiation," Proc. Roy. Soc. A. (London) 122, 647 (1929).

J. R. Greening, Fundamentals of radiation dosimetry (A. Hilger in collaboration with The Hospital Physicists' Association, Bristol, England, 1981).

J. A. Halblieb and T. A. Mehlhorn, "ITS: The Integrated TIGER Series of coupled electron/photon Monte Carlo transport codes," Sandia National Laboratory Report SAND 84-0573 (1984).

Stuart Herbert, Generic NQS v3.5 (http://www.gnqs.org/, Sheffield, UK, 1994-2000). 
A. K. Ho and B. R. Paliwal, "Stopping-power and mass energy-absorption coefficient ratios for Solid Water," Med. Phys. 13 (3), 403-404 (1986).

A. K. Ho, B. R. Paliwal, and F. H. Attix, "Charge storage in electron-irradiated phantom materials," Med. Phys. 13 (1), 99-100 (1986).

J. G. Holt, A. Buffa, D. J. Perry et al., "Absorbed dose measurements using parallel plate polystyrene ionization chambers in polystyrene phantoms," Int. J. Radiat. Oncol. Biol. Phys. 5 (11-12), 2031-2038. (1979).

John Howard Hubbell, "Photon cross sections, attenuation coefficients, and energy absorption coefficients from $10 \mathrm{keV}$ to $100 \mathrm{GeV}$," Report NSRDS-NBS29, U.S. National Bureau of Standards, Washington D. C. (1969).

John Howard Hubbell, "Photon mass attenuation and energy-absorption coefficients from $1 \mathrm{keV}$ to $20 \mathrm{MeV}, ”$ Int. J. Appl. Rad. Isot. 33, 1269 (1982).

John Howard Hubbell, H. A. Gimm, and I. $\emptyset$ verb $\varnothing$, "Pair, triplet and total cross sections for $1 \mathrm{MeV}-100 \mathrm{GeV}$ photons in elements Z = 1-100.," J. Phys. Chem. Ref. Data 9, 1023 (1980)

John Howard Hubbell, W. J. Veigele, E. A. Briggs et al., "Atomic form factors, incoherent scattering functions and photon scattering cross section," J. Phys. Chem. Ref. Data 4, 471 (1975)

M. A. Hunt, G. J. Kutcher, and A. Buffa, "Electron backscatter corrections for parallel-plate chambers," Med. Phys. 15 (1), 96-103. (1988). $(199,202)$

International Atomic Energy Agency, Absorbed dose determination in photon and electron beams: An international code of practice (IAEA Technical Reports Series No. 277, Vienna, 1987). $(88,92,101,187)$ 
International Atomic Energy Agency, Absorbed Dose Determination in External Beam Radiotherapy based on Absorbed-Dose-to-Water Standards: An international Code of Practice for Dosimetry, Draft V.7 (IAEA Technical Reports Series No. 398, Vienna, 2000). $(88,100,101)$

International Atomic Energy Agency, The use of plane parallel ionization chambers in high energy electron and photon beams: An international code of practice for dosimetry (IAEA Technical Reports Series No. 381, Vienna, 1997). $(100,101)$

ICRU, "Radiation quantities and units," ICRU Report 19, International Commission on Radiation Units and Measurements, Bethesda, Maryland (1971).

ICRU, "Measurement of absorbed dose in a phantom irradiated by a single beam of $\mathrm{X}$ or gamma rays," ICRU Report 23, International Commission on Radiation Units and Measurements, Bethesda, Maryland (1973).

ICRU, "Determination of absorbed dose in a patient irradiated by beams of $\mathrm{X}$ or gamma rays in radiotherapy procedures," ICRU Report 24, International Commission on Radiation Units and Measurements, Washington D.C. (1976). $(87,88,186)$

ICRU, "Radiation quantities and units," ICRU Report 33, International Commission on Radiation Units and Measurements, Washington, D.C. (1980). $(1,10,48,72,74,75)$

ICRU, "The dosimetry of pulsed radiation," ICRU Report 34, International Commission on Radiation Units and Measurements, Washington, D.C. (1982).

ICRU, "Stopping powers for electrons and positrons," ICRU Report 37, International Commission on Radiation Units and Measurements, Bethesda, Maryland (1984). $(11,61,62,63,65,67,133,141,154,190,191)$ 
Institute of Physics \& Engineering in Medicine \& Biology, "The IPEMB code of practice for the determination of absorbed dose for $\mathrm{x}$ - rays below $300 \mathrm{kV}$ generating potential ( $0.035 \mathrm{~mm} \mathrm{Al}-4 \mathrm{~mm} \mathrm{Cu} \mathrm{HVL} ; 10-300 \mathrm{kV}$ generating potential). Institution of Physics and Engineering in Medicine and Biology," Phys. Med. Biol. 41 (12), 2605-2625 (1996).

Institute of Physics \& Engineering in Medicine \& Biology, "The IPEMB code of practice for electron dosimetry for radiotherapy beams of initial energy from 2 to $50 \mathrm{MeV}$ based on an air kerma calibration. Institution of Physics and Engineering in Medicine and Biology," Phys. Med. Biol. 41 (12), 2557-2603 (1996).

John David Jackson, Classical Electrodynamics, 3rd ed.(John Whiley, New York, 1999).

F. James, "RANLUX: A FORTRAN implementation of the high-quality pseudo-random number generator of Lüsher," Computer Physics Communications 79, 111-114 (1994).

F. James, "A review of pseudorandom number generators," CERN-Data Handling Division, Report DD/88/22 (1988).

Theodore M. Jenkins, Walter R. Nelson, and Alessandro Rindi, Monte Carlo transport of electrons and photons (Plenum Press, New York, 1988).

Harold Elford Johns and John Robert Cunningham, The physics of radiology, 4th ed. (Charles C. Thomas, Springfield, Ill., U.S.A., 1983). $(6,37,78,111)$

Malvin H. Kalos and Paula A. Whitlock, Monte Carlo methods (J. Wiley \& Sons, New York, 1986). 
C. J. Karzmark, Craig S. Nunan, and Eiji Tanabe, Medical electron accelerators (McGraw-Hill Inc. Health Professions Division, New York, 1993).

I. Kawrakow, "Accurate condensed history Monte Carlo simulation of electron transport. I. EGSnrc, the new EGS4 version," Med. Phys. 27 (3), 485-498. (2000).

I. Kawrakow, "Accurate condensed history Monte Carlo simulation of electron transport. II. Application to ion chamber response simulations," Med. Phys. 27 (3), 499-513. (2000). $(132,135)$

I. Kawrakow, M. Fippel, and K. Friedrich, "3D electron dose calculation using a Voxel based Monte Carlo algorithm (VMC)," Med. Phys. 23 (4), 445-457 (1996).

I. Kawrakow and D. W. O. Rogers, "The EGSnrc Code System: Monte Carlo simulation of electron and photon transport," Technical Report PIRS-701, National Research Concil of Canada, Ottawa, Canada (2000). $(132,135)$

Faiz M. Khan, The physics of radiation therapy, 2nd ed. (Williams \& Wilkins, Baltimore, 1994). $(6,9)$

Faiz M. Khan, K. P. Doppke, K. R. Hogstrom et al., "Clinical electron-beam dosimetry: report of AAPM Radiation Therapy Committee Task Group No. 25," Med. Phys. 18 (1), 73-109 (1991).

O. Klein and Y. Nishina, "Über die Streuung von Strahlung durch freie Elektronen nach der neuen relativistischen Quantendynamik von Dirac," Physik 52, 853-868 (1929). 
S. C. Klevenhagen, "Determination of absorbed dose in high-energy electron and photon radiation by means of an uncaibrated ionization chamber," Phys. Med. Biol. 36 (2), 239-253 (1991).

S. C. Klevenhagen, "Implications of electron backscatter for electron dosimetry," Phys. Med. Biol. 36, 1013-1018 (1991).

$(199,202)$

S. C. Klevenhagen, "Electron backscattering. Implication to electron dosimetry," Radiol Med (Torino) 80 (4 Suppl 1), 160-162. (1990). $(199,202)$

D. E. Knuth, "Seminumerical algorithms," in The art of computer programming (Addison Wesley, Reading Mass., 1981), Vol. II.

D. E. Knuth, "Seminumerical algorithms," in The art of computer programming (Addison Wesley, Reading Mass., 1997), Vol. II.

P. S. Laplace, "Theorie analytique des probabilités, Livre 2," in Oeuvres complètes de Laplace (L'académie des Sciences, Paris, 1886), Vol. 7, part 2, pp. 365-366.

C. Michael Lederer, Virginia S. Shirley, and Edgardo Browne, Table of isotopes, 7th / ed. (Wiley, New York, 1978).

M. S. Livingston and H. A. Bethe, "Nuclear physics," Rev. Mod. Phys. 9, 245 (1937).

R. Loevinger, "Extrapolation chamber for the measurement of beta sources," Sci. Instrum. 24, 907-914 (1953).

M. Lüscher, "A portable high-quality random number generator for lattice field theory simulations," Computer Physics Communications 79, 100-110 (1994). 
C. M. Ma, E. Mok, A. Kapur et al., "Clinical implementation of a Monte Carlo treatment planning system," Med. Phys. 26 (10), 2133-2143 (1999).

C. M. Ma and D. W. Rogers, "Monte Carlo calculated wall correction factors for plane-parallel chambers in high-energy electron beams," Med. Phys. (abstract), 22, 672 (1995).

$(200,202)$

T.R. Mackie, P. Reckwerdt, T. McNutt et al., "Photon beam dose computations," in Teletherapy Physics, Present and Future, edited by J.R. Palta and T.R. Mackie (Advanced Medical Publishing, Madison, WI, 1996), pp. 103-174.

D. J. Manson, D. Velkley, J. A. Purdy et al., "Measurements of surface dose using build-up curves obtained with an extrapolation chamber," Radiology 115 (2), 473-474. (1975).

Pierre Marmier and Eric Sheldon, Physics of nuclei and particles (Academic Press, New York, 1969). $(35,58,61)$

G. Marsaglia and A. Zaman, "Toward a universal random number generator," Statistics and Probability letters 8, 35-39 (1990).

G. Marsaglia and A. Zaman, "A new class of random number generators," Annals of Applied Probability 1, 462-480 (1991). .$(131,134)$

William Herbert McMaster and Lawrence Radiation Laboratory., Compilation of $x$-ray cross sections (University of California Lawrence Radiation Laboratory, Livermore, California, 1969).

G. Z. Molière, "Theorie der Streuung schneller geladener Teilchen I: Einzelstreuung am abgeschirmten Coulomb-Feld," Z. Naturforsch 2a, 133 (1947). 
G. Z. Molière, "Theorie der Steuung schneller geladener Teilchen. II. Mehrfach-und Vielfachstreuung," Z. Naturforsch 3a, 97 (1948).

N. F. Mott, "The Scattering of Fast Electrons by Atomic Nuclei," Proc. Roy. Soc. A. A124, 426 (1929).

A. E. Nahum, "Extension of the Spencer-Attix Cavity Theory to the 3 media situation for Electron beams," in Dosimetry in Radiotherapy, edited by IAEA (IAEA, Vienna, 1988), Vol. 1, pp. 87-115. $(86,199)$

A. E. Nahum, "Water/Air Mass Stopping Power Ratios for Megavoltage Photon and Electron Beams," Phys. Med. Biol. 23, 24 (1978).

W. R. Nelson, H. Hirayama, and D. W. O. Rogers, "The EGS4 Code system," Stanford Linear Accelerator Center Report SLAC-256 (Stanford Calif., 1985).

NOMOS Corporation, PEREGRINE (NOMOS Corporation, Sewickley, PA, 2000).

M. Olivares, F. DeBlois, E. B. Podgorsak, and J. P. Seuntjens, "Electron Fluence Correction Factors for Various Materials in Clinical Electron Beams," Med. Phys. 28 (8), 1727-1734. (2001).

E. F. Plechaty, D. E. Cullen, and R. J. Howerton, Tables and graphs of photon interaction cross sections form $1.0 \mathrm{keV}$ to $100 \mathrm{MeV}$ derived from $\mathrm{LLL}$ evaluated nuclear data library, UCRL-50400 (University of California, Lawrence Livermore Laboratory, Springfield, 1975).

E. B. Podgorsak, P. Metcalfe, and J. Van Dyk, "Medical Accelerators," in The modern technology of radiation oncology: a compendium for medical physicists and radiation 
oncologists, edited by J. Van Dyk (Medical Physics Pub., Madison, Wis., 1999), pp. $349-435$.

R. H. Pratt, "Atomic Photoelectric Effect at High Energies," Phys. Rev. 117, 1017-1102 (1960).

J. A. Rawlinson, A. F. Bielajew, P. Munro et al., "Theoretical and experimental investigation of dose enhancement due to charge storage in electron-irradiated phantoms," Med. Phys. 11 (6), 814-821 (1984).

C. S. Reft, "Output calibration in solid water for high energy photon beams," Med. Phys. 16 (2), 299-301 (1989).

Richard Rhodes, Dark sun: the making of the hydrogen bomb (Simon \& Schuster, New York, 1995).

Richard Rhodes, The making of the atomic bomb (Simon \& Schuster, New York, 1986).

D. W. O. Rogers, "Fundamentals of dosimetry based on absorbed-dose standards," in Teletherapy Physics, Present and Future, edited by J.R. Palta and T.R. Mackie (Advanced Medical Publishing, 1996), pp. 319-356.

$(11,190)$

D. W. O. Rogers, "Ionizing Radiation Dosimetry and Medical Physics," Physics in Canada 51, 178-181 (1995)...

D. W. Rogers, B. A. Faddegon, G. X. Ding et al., "BEAM: a Monte Carlo code to simulate radiotherapy treatment units," Med. Phys. 22 (5), 503-524. (1995). 
D. W. O. Rogers, I. Kawrakow, J. P. Seuntjens et al., "NRC user codes for EGSnrc," Technical Report PIRS-702, National Research Concil of Canada, Ottawa, Canada (2000).

D. W. O. Rogers, I. Kawrakow, and B. R. B. Walters, "Revised relationship between R50 and Eo for electron beams," proceedings of the $42^{\text {nd }}$ scientific meeting of the AAPM, Chicago, July 23-28, TU-E313-1, (2000).

Wilhelm Conrad Röntgen, Adrian Thomas, Ian Isherwood et al., The Invisible Light : 100 years of medical radiology (Blackwell Science Ltd, Oxford; Cambridge, Mass., 1995).

R. J. Schulz, P. R. Almond, G. Kutcher et al., "Clarification of the AAPM Task Group 21 protocol," Med. Phys. 13 (5), 755-759 (1986). (95, 165, 165, 166)

C. G. Soares, "Calibration of ophthalmic applicators at NIST: a revised approach," Med. Phys. 18 (4), 787-793. (1991).

L. V. Spencer and F. H. Attix, "A theory of cavity ionization," Radiat. Res. 3, 239-254 (1955). $(67,84,85,187)$

R. M. Sternheimer, "The Density Effect for the Ionization Loss in Various Materials," Phys. Rev. 88, 851-859 (1952).

R. M. Sternheimer, "Denșity Effect for the Ionization Loss of Charged Particles in Various Substances," Atom. Data and Nucl. Data Tables 30, 261 (1984).

E. Storm and H. I. Israel, Photon cross sections from $1 \mathrm{keV}$ to $100 \mathrm{MeV}$ for elements from $Z=1$ to $Z=100$ (Academic Press, New York, 1970). 
J. E. Turner, Atoms, radiation, and radiation protection, 2nd ed. (J. Wiley, New York, 1995).

S. M. Ulam and J. von Newmann, "On combination of stochastic and deterministic processes," Bull. Amer. Math. Soc. 53, 1120 (1947).

J. Van Dyk, R.B. Barnett, and J.J. Battista, "Computerized Radiation Treatment Planning Systems," in The modern technology of radiation oncology: a compendium for medical physicists and radiation oncologists, edited by J. Van Dyk (Medical Physics Pub., Madison, Wis., 1999), pp. 231-286.

A. A. Varfolomeev and I. A. Svetlolobov, "Monte Carlo calculations of electromagnetic cascades with account of the influence of the medium on bremsstrahlung," Soviet Physics JETP 36, 1263-1270 (1959).

K. Weaver, C. H. Siantar, W. Chandler et al., "A source model for efficient brachytherapy computations with Monte Carlo," Med. Phys. 23 (12), 2079-2084 (1996).

M. S. Weinhous and J. A. Meli, "Determining Pion, the correction factor for recombination losses in an ionization chamber," Med. Phys. 11 (6), 846-849 (1984). $(95,166)$

George Neilson Whyte, Principles of radiation dosimetry (Wiley, New York, 1959).

E. J. Williams, "The passage of $\alpha$ and $\beta$ particles through matter and Born's Theory of collisions," Proc. Roy. Soc. A. 135, 108-142 (1932).

Francis $\mathrm{H}$. Williams, The Roentgen rays in medicine and surgery: as an aid in diagnosis and as a therapeutic agent designed for the use of practitioners and students (Macmillan, New York, 1901). 
R. R. Wilson, "Monte Carlo study of shower production," Phys. Rev. 86, 261-269 (1952).

H. Wyckoff and F. H. Attix, "Design of free-air ionization chambers," Handbook 64, National Bureau of Standards, Washington, D.C., (1957).

C. L. Yang, D. W. Rogers, K. R. Shortt et al., "Ion recombination in ion chambers in continuous radiation," proceedings of the $45^{\text {th }}$ scientific meeting of the COMP, Sherbrooke, June 16-19, 23-25 (1999).

$(123,163,174)$

C. E. Zankowski, "Calibration of photon and electron beams with an extrapolation chamber," Ph.D. Thesis, McGill University, 1997.

$(15,108,113,163,187,198,198,200,209)$

C. E. Zankowski and E. B. Podgorsak, "Calibration of photon and electron beams with an extrapolation chamber," Med. Phys. 24 (4), $497-503$ (1997).

$(15,108,187,198,209)$

C. E. Zankowski and E. B. Podgorsak, "Determination of saturation charge and collection efficiency for ionization chambers in continuous beams," Med. Phys. 25 (6), 908-915 (1998) $(95,108,123,124,163,169,171)$

C. E. Zankowski and E. B. Podgorsak, "Ionization gradient chamber in absolute photon and electron dosimetry,: Radiology and Oncology (Slovenia) 30, 138-141 (1996). ...(108, 187, 198, 209)

C. E. Zankowski, S. Vatnitsky, J. Siebers et al., "Proton beam output measurement with an extrapolation chamber," Med. Dosim. 23 (4), 288-291 (1998). 